\title{
Phosphine-Catalyzed Asymmetric Cycloaddition Reaction of Diazenes: Enantioselective Synthesis of Chiral Dihydropyrazoles
}

Chang Wang, Yuehua Chen, Jingyu Li, Leijie Zhou, Bo Wang, Yumei Xiao, and Hongchao Guo*

Email: hchguo@cau.edu.cn

Table of Contents

$\begin{array}{ll}\text { General Information } & \text { S1 }\end{array}$

$\begin{array}{ll}\text { General Procedure for Synthesis of Substrates } 1 \text { and } 2 & \text { S1 }\end{array}$

General Procedure for Preparation of Racemic [3+2] Products $\quad$ S2

General Procedure for Preparation of Chiral [3+2] Products $\quad$ S3

Scaled-up Synthesis and Further Transformations of 3ah $\quad$ S3

Characterization Data of the Products 3, 4, 5, $6 \quad$ S5

$\begin{array}{lr}\text { Copies of }{ }^{1} \mathrm{H} \text { and }{ }^{13} \mathrm{C} \text { NMR Spectra } & \text { S47 }\end{array}$

$\begin{array}{lr}\text { X-Ray Crystallographic Data of 3ha } & \text { S89 }\end{array}$ 


\section{General Information}

All reactions were performed under Ar atmospheres in oven-dried glassware with magnetic stirring. Unless otherwise stated, all reagents were purchased from commercial suppliers and used without further purification. All solvents were purified and dried according to standard methods prior to use. Organic solutions were concentrated under reduced pressure on a rotary evaporator or an oil pump. Reactions were monitored through thin layer chromatography (TLC) on silica gel-precoated glass plates. Chromatograms were visualized by fluorescence quenching with UV light at $254 \mathrm{~nm}$. Flash column chromatography was performed using Qingdao Haiyang flash silica gel (200-300 mesh). Infrared spectra were recorded using a Bruker Optics TENSOR 27 instrument. ${ }^{1} \mathrm{H}$ and ${ }^{13} \mathrm{C}$ NMR spectra were recorded in $\mathrm{CDCl}_{3}$ using a 300, 400 or $600 \mathrm{MHz} \mathrm{NMR}$ instrument (referenced internally to $\mathrm{Me}_{4} \mathrm{Si}$ ). ${ }^{1} \mathrm{H}$ NMR data are reported as follows: chemical shift, multiplicity $(\mathrm{s}=$ singlet; $\mathrm{d}=$ doublet; $\mathrm{q}=$ quartet; $\mathrm{m}=$ multiplet; $\mathrm{br}=$ broad), coupling constant $(\mathrm{Hz})$, and integral. Data for ${ }^{13} \mathrm{C}$ NMR spectra are reported in terms of chemical shift. Optical rotation was obtained on a Perkin-Elmer 343 polarimeter. Melting points were determined by a X-4 digital micro melting point apparatus. Accurate mass measurements were performed using an Agilent instrument with the ESI-MS technique. HPLC analysis was performed on Agilent 1220 series, UV detection monitored at $254 \mathrm{~nm}$, using a CHIRALPAK IC and ID column with hexane and $i-\mathrm{PrOH}$ as the eluent. X-ray crystallographic data were collected using a Gemini E Rigaku.

\section{Synthesis of Substrates 1 and 2}

A: The substrates 1 were synthesized according to previous reported protocols. ${ }^{1}$

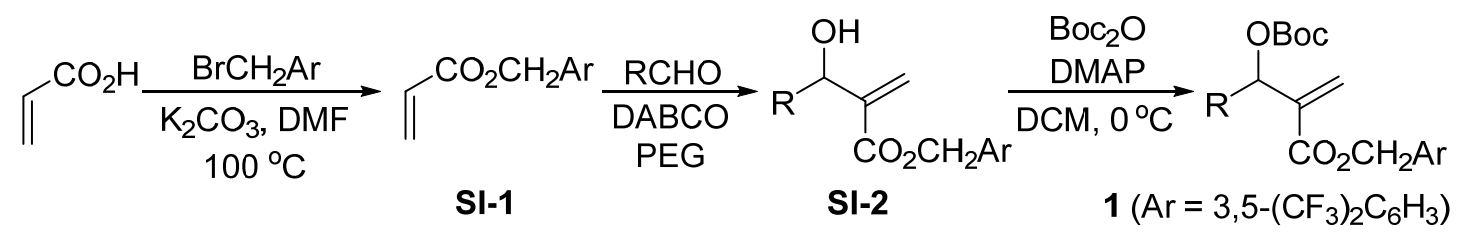

Under nitrogen atmosphere, a mixture of acrylic acid (5.4 g, $75 \mathrm{mmol})$ and $\mathrm{K}_{2} \mathrm{CO}_{3}(10.35 \mathrm{~g})$ in DMF $(125 \mathrm{~mL})$ was stirred at $0{ }^{\circ} \mathrm{C}$ for $45 \mathrm{~min}$. After slowly adding 3,5 bis(trifluoromethyl)benzylbromide $(19.35 \mathrm{~g}, 63 \mathrm{mmol})$, the reaction mixture was stirred at

\footnotetext{
1 Yang, W.; Sun, W.; Zhang, C.; Wang, Q.; Guo, Z.; Mao, B.; Liao, J.; Guo H. ACS Catal. 2017, 7, 3142.
} 
$100{ }^{\circ} \mathrm{C}$ for $24 \mathrm{~h}$. The reaction mixture was added into water $(1 \mathrm{~L})$ and the resulting mixture was then extracted with $\mathrm{Et}_{2} \mathrm{O}(250 \mathrm{~mL} \times 2)$. The organic layer was dried over $\mathrm{Na}_{2} \mathrm{SO}_{4}$, concentrated, and purified by flash column to provide the intermediate SI-1.

A mixture of SI-1 (2.98 g, $10 \mathrm{mmol})$, aldehyde $(12 \mathrm{mmol})$ and triethylene diamine $(1.12 \mathrm{~g}, 10$ mmol) in polyethylene glycol was stirred for $24 \mathrm{~h}$. The resulting reaction mixture was diluted with $\mathrm{Et}_{2} \mathrm{O}(50 \mathrm{~mL})$ and washed with water. The organic layer was dried over $\mathrm{Na}_{2} \mathrm{SO}_{4}$, concentrated, and purified by column chromatography to provide the intermediate SI-2. The mixture of dibutyldicarbonate $(1.31 \mathrm{~g}, 6 \mathrm{mmol})$ and 4-dimethylaminopyridine $(122 \mathrm{mg}, 1$ mmol) in DCM (10 mL) was slowly added (30 $\mathrm{min})$ to the solution of SI-2 (5 mmol, 1 equiv.) in DCM $(10 \mathrm{~mL})$. The mixture was stirred at $0{ }^{\circ} \mathrm{C}$ for $5 \mathrm{~h}$. The resulting reaction mixture was washed with $1 \mathrm{~N}$ aqueous $\mathrm{HCl}$, saturated aqueous $\mathrm{NaHCO}_{3}$, and brine. The organic layer was dried over $\mathrm{Na}_{2} \mathrm{SO}_{4}$, concentrated and purified by column chromatography to provide Morita-Baylis-Hillman carbonates 1.

B: The substrates $\mathbf{2}$ were synthesized according to previous reported protocols. ${ }^{2}$

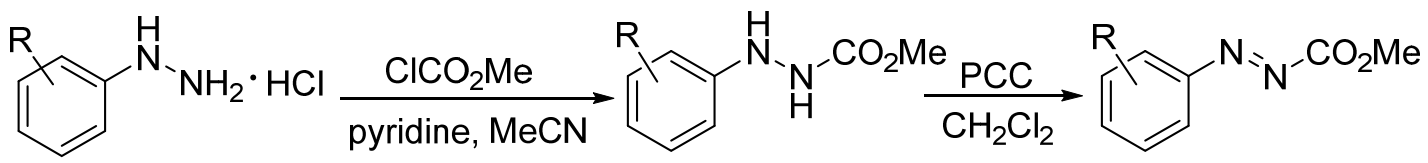
SI-3

To the solution of aryl hydrazine hydrochloride $(10 \mathrm{mmol})$ in $\mathrm{CH}_{3} \mathrm{CN}(20 \mathrm{~mL})$, Pyridine $(2.2$ equiv., $22 \mathrm{mmol})$ was added. The chloroformate (1.1 equiv., $11 \mathrm{mmol})$ was then added dropwise under stirring at $0{ }^{\circ} \mathrm{C}$. The reaction mixture was stirred for another $15 \mathrm{~min}$ and subsequently for $1 \mathrm{~h}$ at room temperature. Water $(20 \mathrm{~mL})$ was added and the resulting mixture was acidified with $\mathrm{HCl}(6 \mathrm{M})$ to $\mathrm{pH} 4-6$. The product was extracted with $\mathrm{CH}_{2} \mathrm{Cl}_{2}(2 \times 20 \mathrm{~mL})$. The combined organic layers were washed with saturated $a q . \mathrm{NaHCO}_{3}(30 \mathrm{~mL})$, brine $(30$ $\mathrm{mL}$ ), dried over $\mathrm{Na}_{2} \mathrm{SO}_{4}$, and the solvent was concentrated under reduced pressure. The crude product was used without further purification.

PCC (1.0 equiv., $10 \mathrm{mmol}$ ) was added to a solution of SI-3 in $40 \mathrm{~mL}$ DCM. The mixture was stirred until the starting material was completely consumed (monitored by TLC). The reaction mixture was filtered. The filtrate was concentrated under reduced pressure and purified by chromatography on silica gel eluted with PE/EA (30/1) to afford the diazenes 2.

2 Qi, L.-W.; Mao, J.-H.; Zhang, J.; Tan, B. Nature Chem. 2017. 10, 58-64. 


\section{General Procedure for Preparation of Racemic [3+2] Products}

Under argon atmosphere, to a mixture of MBH carbonates $1(0.15 \mathrm{mmol})$, methyl aryldiazene carbonates $2(0.1 \mathrm{mmol})$ and catalyst $\mathrm{PBu}_{3}(20 \mathrm{~mol} \%, 0.02 \mathrm{mmol})$ in a Schlenk tube, $1 \mathrm{~mL}$ of $\mathrm{MeCN}$ was added at room temperature. The resulting mixture was stirred until the starting material was completely consumed (monitored by TLC) and then was concentrated to dryness. The residue was purified through flash column chromatography (12\% EtOAc / PE) to afford the corresponding cycloaddition product 3 .

\section{General Procedure for Preparation of Chiral [3+2] Products}

Under argon atmosphere, to a mixture of MBH carbonates $1(0.15 \mathrm{mmol})$, methyl aryldiazene carbonates $2(0.1 \mathrm{mmol})$ and chiral catalyst P5 $(20 \mathrm{~mol} \%, 0.02 \mathrm{mmol})$ in a Schlenk tube, $1 \mathrm{~mL}$ of $\mathrm{MeCN}$ was added at $-20{ }^{\circ} \mathrm{C}$. The resulting mixture was stirred until the starting material was completely consumed (monitored by TLC) and then was concentrated to dryness. The residue was purified through flash column chromatography (12\% EtOAc / PE) to afford the corresponding cycloaddition product 3 .

\section{Scaled-up Synthesis of 3ah}

Under argon atmosphere, to a mixture of MBH carbonates $1 \mathrm{a}(0.76 \mathrm{~g}, 1.5 \mathrm{mmol})$, methyl aryldiazene carbonates $2 \mathrm{~h}(0.24 \mathrm{~g}, 1 \mathrm{mmol})$ and chiral catalyst $\mathbf{P 5}(20 \mathrm{~mol} \%, 0.4 \mathrm{mmol})$ in a Schlenk tube, $10 \mathrm{~mL}$ of $\mathrm{MeCN}$ was added at $-20^{\circ} \mathrm{C}$. The resulting mixture was stirred for 10 $\mathrm{h}$ until the starting material was completely consumed (monitored by TLC) and then was concentrated to dryness. The residue was purified through flash column chromatography $(12 \%$ EtOAc / PE) to afford the corresponding cycloaddition product 3 in $87 \%$ yield $(0.55 \mathrm{~g})$ with $97 \%$ ee.

\section{Transformations of the Product 3ah}<smiles>CC(=O)N1C=C(C(=O)OCc2cc(C(F)(F)F)cc(C(F)(F)F)c2)[C@H](c2ccccc2)N1c1ccccc1Br</smiles><smiles>CC(=O)N1C=C(C(=O)OCc2cc(C(F)(F)F)cc(C(F)(F)F)c2)[C@](c2ccccc2)(c2ccc(Cl)cc2)N1c1ccccc1</smiles>

4, $43 \%$ yield, $96 \%$ ee 
Condition a: Under argon atmosphere, to a mixture of [3+2] cycloadduct $\mathbf{3 a h}(0.1 \mathrm{mmol}, 63$ $\mathrm{mg})$, 4- $\mathrm{ClC}_{6} \mathrm{H}_{4} \mathrm{~B}(\mathrm{OH})_{2}(0.2 \mathrm{mmol}, 32 \mathrm{mg}), \mathrm{Cs}_{2} \mathrm{CO}_{3}(0.2 \mathrm{mmol}, 65 \mathrm{mg})$ and catalyst $\mathrm{Pd}(\mathrm{PPh})_{4}$ (5 mol\%, $0.005 \mathrm{mmol}, 2.7 \mathrm{mg}$ )/butyl-di(1-adamantyl)phosphine (6 mol \%, $0.006 \mathrm{mmol}, 2.2$ $\mathrm{mg}$ ) in a Schlenk tube, $1 \mathrm{~mL}$ of DCE was added. The resulting mixture was stirred at $60{ }^{\circ} \mathrm{C}$ for $24 \mathrm{~h}$ until the starting material was completely consumed (monitored by TLC) and then was concentrated to dryness. The residue was purified through flash column chromatography (12\% EtOAc / PE) to afford the corresponding cycloaddition product 4 in $43 \%$ yield ( $28 \mathrm{mg})$ with $96 \%$ ee.<smiles>CC(=O)N1C=C(C(=O)OCc2cc(C(F)(F)F)cc(C(F)(F)F)c2)C(c2ccccc2)[C@H]1c1ccccc1Br</smiles>

3ah

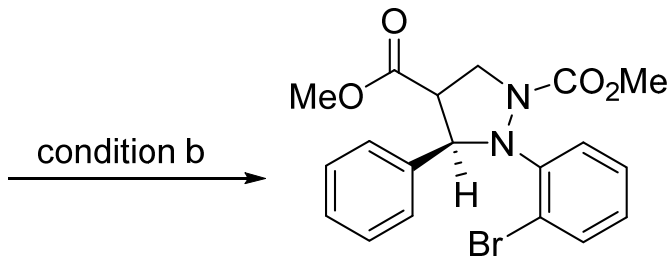

5, $67 \%$ yield, $96 \%$ ee

Condition b: To the mixture of cycloaddition product 3ah $(0.1 \mathrm{mmol}, 63 \mathrm{mg})$ with $1 \mathrm{~mL}$ of THF, a solution of $\mathrm{SmI}_{2}$ in $\mathrm{MeOH}(0.1 \mathrm{M}, 4 \mathrm{~mL})$ was added dropwise. The resulting mixture was stirred at room temperature for 10 minutes and quenched with aqueous $\mathrm{NaHCO}_{3}$ solution. The aqueous layer was extracted with EA three times. The combined organic layers were concentrated in vacuo. The resulting residue was purified by flash chromatography on silica gel (eluting with PE:EA = 7:1) to afford 5 (28 mg, 67\% yield, 96\% ee).<smiles>CC(=O)N1C=C(C(=O)OCc2cc(C(F)(F)F)cc(C(F)(F)F)c2)[C@H](c2ccccc2)N1c1ccccc1Br</smiles>

3ah<smiles>CC(=O)N1CC(C(=O)OCc2cc(C(F)(F)F)cc(C(F)(F)F)c2)[C@@H](c2ccccc2)N1c1ccccc1Br</smiles>

6, $37 \%$ yield, $96 \%$ ee

Condition c: To the mixture of cycloaddition product $3 \mathbf{a h}(0.1 \mathrm{mmol}, 63 \mathrm{mg})$ with $1 \mathrm{~mL}$ THF, a solution of DIBAL-H in THF $(1 \mathrm{M}, 1 \mathrm{~mL})$ was added dropwise. The resulting mixture was stirred at room temperature for 10 minutes and quenched with aqueous $\mathrm{NH}_{4} \mathrm{Cl}$ solution. The aqueous layer was extracted with ethyl acetate three times. The combined organic layers were concentrated in vacuo. The resulting residue was purified by flash chromatography on silica gel (eluting with PE:EA = 7:1) to afford 6 (23 mg, 37\% yield, 96\% ee). 


\section{Characterization Data of the Products 3, 4, 5}

(S)-4-(3,5-bis(trifluoromethyl)benzyl) 1-methyl-2,3-diphenyl-2,3-dihydro-1H-pyrazole1,4-dicarboxylate.<smiles>CC(=O)N1C=C(C(=O)OCc2cc(C(F)(F)F)cc(C(F)(F)F)c2)[C@H](c2ccccc2)N1c1ccccc1</smiles>

White solid, 99\% yield (54 mg). Purified by flash chromatography (12\% EtOAc/PE). mp = $130-133{ }^{\circ} \mathrm{C} ;[\alpha]{ }_{\mathrm{D}}^{20}=+110.4\left(c 0.54, \mathrm{CH}_{2} \mathrm{Cl}_{2}\right) ;{ }^{1} \mathrm{H}$ NMR $\left(300 \mathrm{MHz}, \mathrm{CDCl}_{3}\right) \delta 8.02(\mathrm{~d}, J=$ $1.1 \mathrm{~Hz}, 1 \mathrm{H}), 7.84(\mathrm{~s}, 1 \mathrm{H}), 7.66(\mathrm{~s}, 2 \mathrm{H}), 7.49-7.31(\mathrm{~m}, 7 \mathrm{H}), 7.19-6.99(\mathrm{~m}, 3 \mathrm{H}), 5.45(\mathrm{~d}, J=$ $1.1 \mathrm{~Hz}, 1 \mathrm{H}), 5.26(\mathrm{~d}, J=13.1 \mathrm{~Hz}, 1 \mathrm{H}), 5.09$ (d, $J=13.1 \mathrm{~Hz}, 1 \mathrm{H}), 3.87(\mathrm{~s}, 3 \mathrm{H}) ;{ }^{13} \mathrm{C}$ NMR $(101$ $\left.\mathrm{MHz}, \mathrm{CDCl}_{3}\right) \delta 162.6,151.9,151.4,140.5,138.2,136.5,131.9(\mathrm{q}, 2 J \mathrm{C}-\mathrm{F}=33.5 \mathrm{~Hz}), 129.4$, 129.0, 128.5, $128.1(\mathrm{q}, 3 J \mathrm{C}-\mathrm{F}=4.0 \mathrm{~Hz}), 126.6,124.1,123.1(\mathrm{q}, 2 J \mathrm{C}-\mathrm{F}=272.4 \mathrm{~Hz}), 122.2$ $(\mathrm{sep}, 3 J \mathrm{C}-\mathrm{F}=3.6 \mathrm{~Hz}), 116.8,112.8,77.1,64.6,54.3$; IR (film) $v_{\max } 3058,2359,1709,1624$, 1490, 1446, 1387, 1358, 1279, 1264, 1178, 1136, 1073, 970, 890, 844, $734 \mathrm{~cm}^{-1}$; HRMS (ESI) calcd for $\mathrm{C}_{27} \mathrm{H}_{21} \mathrm{~F}_{6} \mathrm{~N}_{2} \mathrm{O}_{4}{ }^{+}[\mathrm{M}+\mathrm{H}]^{+}$551.1400, found 551.1413. HPLC analysis: $97 \%$ ee $($ CHIRALPAK IC, isopropanol/hexane $=10: 90,1.0 \mathrm{~mL} / \mathrm{min}, \mathrm{UV}: 254 \mathrm{~nm}), t_{\mathrm{R}}=7.639 \mathrm{~min}$ (major), $11.675 \mathrm{~min}$ (minor).
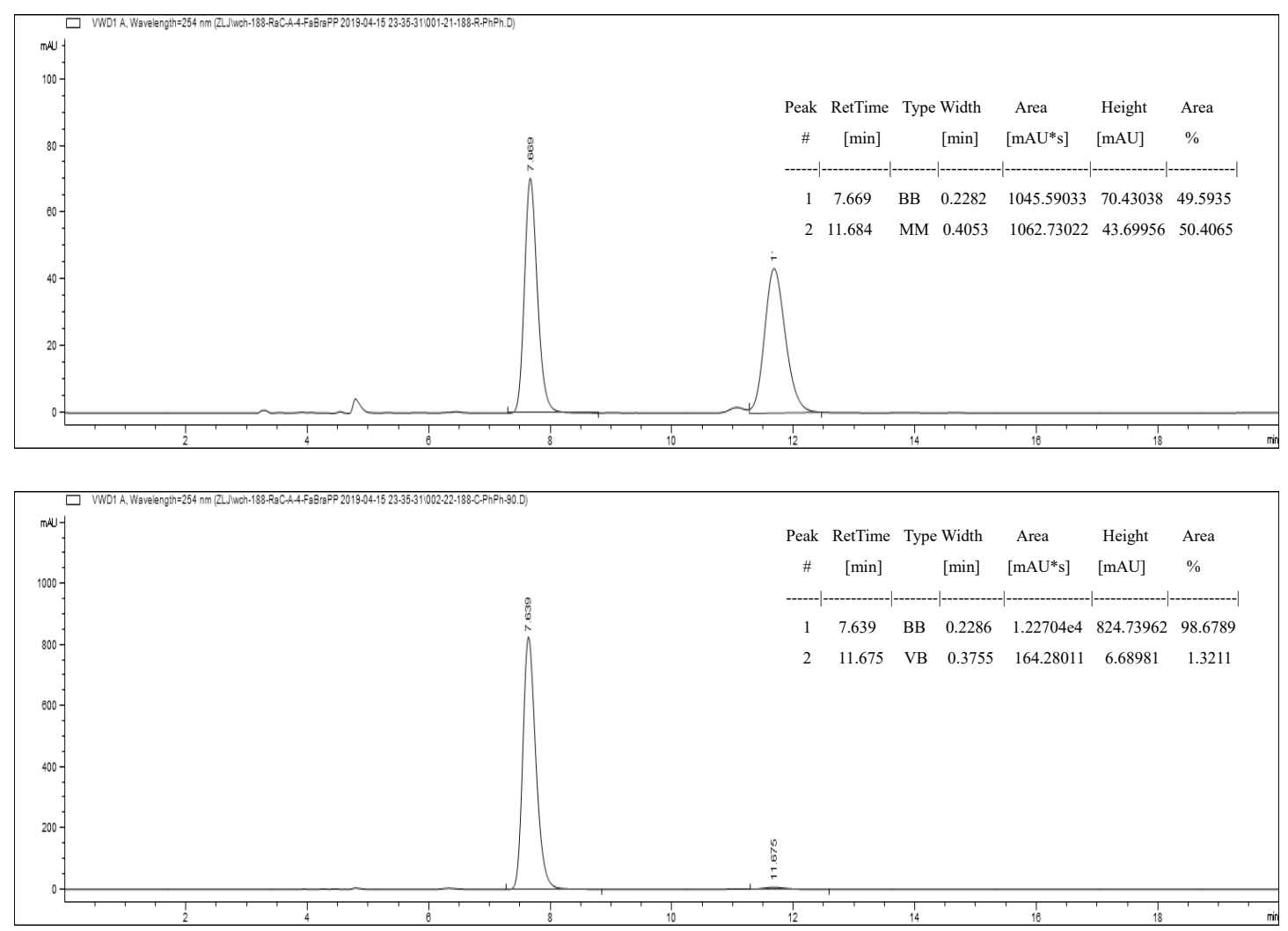
(R)-4-(3,5-bis(trifluoromethyl)benzyl) 1-methyl 3-(2-fluorophenyl)-2-phenyl-2,3dihydro-1H-pyrazole-1,4-dicarboxylate<smiles>CC(=O)N1C=C(C(=O)OCc2cc(C(F)(F)F)cc(C(F)(F)F)c2)[C@@H](c2ccccc2F)N1c1ccccc1</smiles>

Pale yellow solid, 97\% yield (55 mg). Purified by flash chromatography (12\% EtOAc/PE). $\mathrm{mp}=118-123{ }^{\circ} \mathrm{C} ;[\alpha]^{20}{ }_{\mathrm{D}}=+79.7\left(c 0.54, \mathrm{CH}_{2} \mathrm{Cl}_{2}\right) ;{ }^{1} \mathrm{H} \mathrm{NMR}\left(300 \mathrm{MHz}, \mathrm{CDCl}_{3}\right) \delta 8.13(\mathrm{~d}$, $J=1.2 \mathrm{~Hz}, 1 \mathrm{H}), 7.84(\mathrm{~s}, 1 \mathrm{H}), 7.63(\mathrm{~d}, J=1.7 \mathrm{~Hz}, 2 \mathrm{H}), 7.46-7.25(\mathrm{~m}, 4 \mathrm{H}), 7.24-7.04(\mathrm{~m}$, 5H), $5.75(\mathrm{~d}, J=1.2 \mathrm{~Hz}, 1 \mathrm{H}), 5.27(\mathrm{~d}, J=13.1 \mathrm{~Hz}, 1 \mathrm{H}), 5.06(\mathrm{~d}, J=13.1 \mathrm{~Hz}, 1 \mathrm{H}), 3.86(\mathrm{~s}$, $3 \mathrm{H}) ;{ }^{13} \mathrm{C} \mathrm{NMR}\left(101 \mathrm{MHz}, \mathrm{CDCl}_{3}\right) \delta 162.2,160.4(\mathrm{~d}, 1 J \mathrm{C}-\mathrm{F}=248.6 \mathrm{~Hz}), 152.2,151.3,138.1$, $137.7,131.9(\mathrm{q}, 2 J \mathrm{C}-\mathrm{F}=33.5 \mathrm{~Hz}), 130.4(\mathrm{~d}, 3 J \mathrm{C}-\mathrm{F}=8.5 \mathrm{~Hz}), 129.4,128.7(\mathrm{~d}, 3 J \mathrm{C}-\mathrm{F}=3.8$ $\mathrm{Hz}), 128.0(\mathrm{q}, 3 J \mathrm{C}-\mathrm{F}=4.2 \mathrm{~Hz}), 127.3(\mathrm{~d}, 2 J \mathrm{C}-\mathrm{F}=12.8 \mathrm{~Hz}), 124.7(\mathrm{~d}, 4 J \mathrm{C}-\mathrm{F}=3.5 \mathrm{~Hz}), 124.2$, $123.1(\mathrm{q}, 1 J \mathrm{C}-\mathrm{F}=274.8 \mathrm{~Hz}), 122.2(\mathrm{sep}, 3 J \mathrm{C}-\mathrm{F}=3.8 \mathrm{~Hz}), 116.8,116.1(\mathrm{~d}, 2 J \mathrm{C}-\mathrm{F}=21.9 \mathrm{~Hz})$, 111.2, 71.6, 64.6, 54.3; IR (film) $v_{\max } 3057,2359,1713,1625,1491,1446,1387,1357,1322$, 1264, 1176, 1136, 1112, 1075, 970, 891, 844, 735, $704 \mathrm{~cm}^{-1}$; HRMS (ESI) calcd for $\mathrm{C}_{27} \mathrm{H}_{20} \mathrm{~F}_{7} \mathrm{~N}_{2} \mathrm{O}_{4}{ }^{+}[\mathrm{M}+\mathrm{H}]^{+}$569.1306, found 569.1295. HPLC analysis: 96\% ee (CHIRALPAK $\mathrm{ID}, i$-PrOH/hexane $=10: 90,1.0 \mathrm{~mL} / \mathrm{min}, \mathrm{UV}: 254 \mathrm{~nm}$ ), $t_{\mathrm{R}}=7.787 \mathrm{~min}$ (major), $8.839 \mathrm{~min}$ (minor).
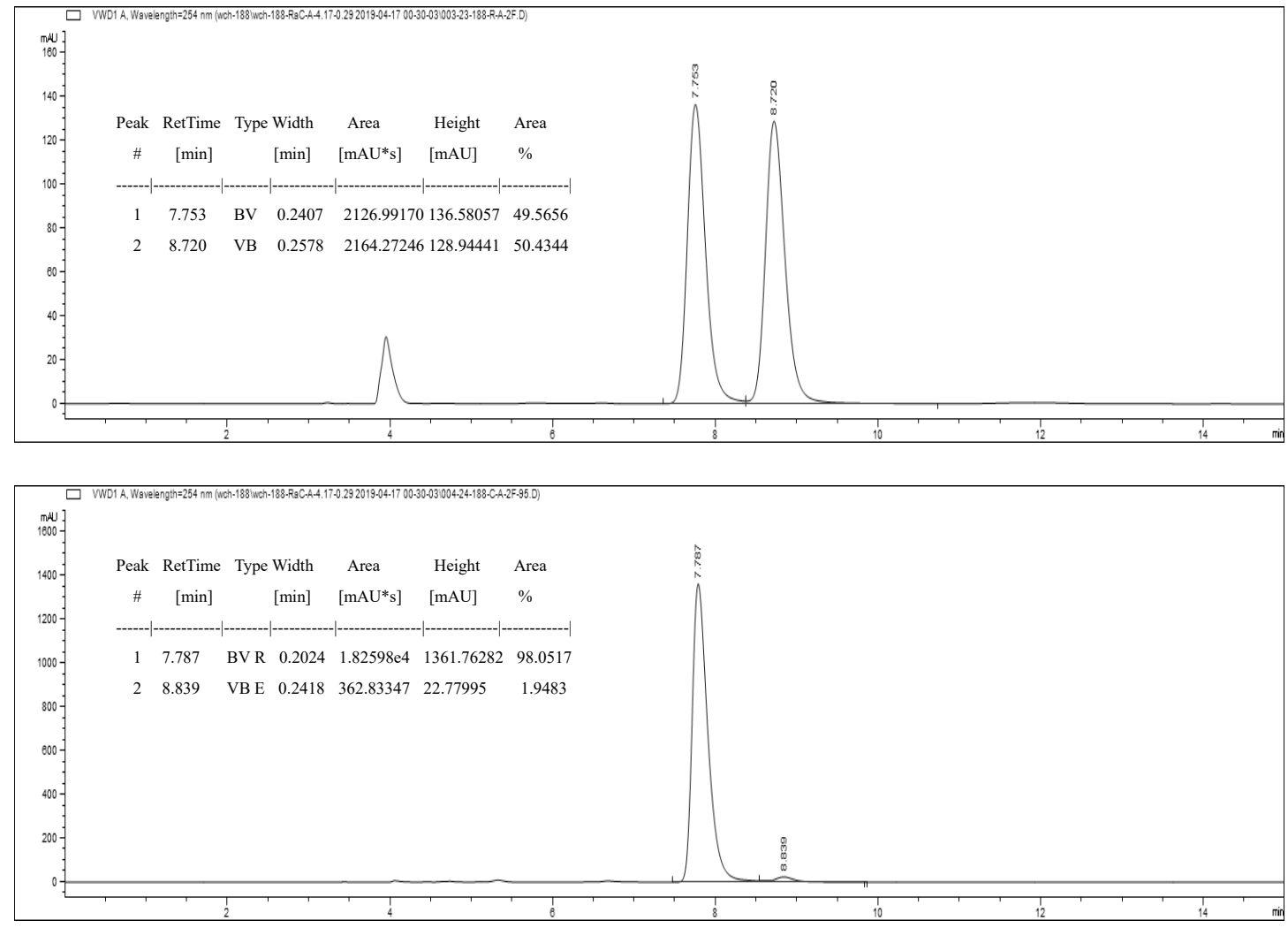
(S)-4-(3,5-bis(trifluoromethyl)benzyl) 1-methyl 3-(3-fluorophenyl)-2-phenyl-2,3-dihydro1H-pyrazole-1,4-dicarboxylate<smiles>CC(=O)N1C=C(C(=O)OCc2cc(C(F)(F)F)cc(C(F)(F)F)c2)C(c2cccc(F)c2)N1c1ccccc1</smiles>

White solid, 95\% yield (54 mg). Purified by flash chromatography (12\% EtOAc/PE). mp $=$ $50-51{ }^{\circ} \mathrm{C} ;[\alpha]{ }_{\mathrm{D}}^{20}=+68.6\left(c 0.54, \mathrm{CH}_{2} \mathrm{Cl}_{2}\right) ;{ }^{1} \mathrm{H}$ NMR $\left(300 \mathrm{MHz}, \mathrm{CDCl}_{3}\right) \delta 8.03(\mathrm{~d}, J=1.1$ $\mathrm{Hz}, 1 \mathrm{H}), 7.87$ (s, 1H), 7.71 (s, 2H), $7.46-7.29$ (m, 4H), $7.25-7.11(\mathrm{~m}, 2 \mathrm{H}), 7.11-6.99(\mathrm{~m}$, $3 \mathrm{H}), 5.47(\mathrm{~s}, 1 \mathrm{H}), 5.30(\mathrm{~d}, J=13.0 \mathrm{~Hz}, 1 \mathrm{H}), 5.13(\mathrm{~d}, J=13.0 \mathrm{~Hz}, 1 \mathrm{H}), 3.89(\mathrm{~s}, 3 \mathrm{H}) ;{ }^{13} \mathrm{C} \mathrm{NMR}$ $\left(75 \mathrm{MHz}, \mathrm{CDCl}_{3}\right) \delta 162.8(\mathrm{~d}, 1 J \mathrm{C}-\mathrm{F}=247.0 \mathrm{~Hz}), 162.1,151.4,150.8,142.7(\mathrm{~d}, 3 J \mathrm{C}-\mathrm{F}=6.5$ $\mathrm{Hz}), 137.9,136.4,131.7(\mathrm{q}, 2 J \mathrm{C}-\mathrm{F}=33.5 \mathrm{~Hz}), 130.1(\mathrm{~d}, 3 J \mathrm{C}-\mathrm{F}=8.1 \mathrm{~Hz}), 129.1,127.8(\mathrm{q}$, $3 J \mathrm{C}-\mathrm{F}=3.0 \mathrm{~Hz}), 124.0,122.8(\mathrm{q}, 1 J \mathrm{C}-\mathrm{F}=271.2 \mathrm{~Hz}), 122.0(\mathrm{sep}, 3 J \mathrm{C}-\mathrm{F}=3.0 \mathrm{~Hz}), 116.5$, $115.0(\mathrm{~d}, 2 J \mathrm{C}-\mathrm{F}=21.4 \mathrm{~Hz}), 113.4(\mathrm{~d}, 2 J \mathrm{C}-\mathrm{F}=22.3 \mathrm{~Hz}), 111.8,76.0,64.3,53.9$; IR (film) $v_{\max }$ 2958, 2359, 1715, 1626, 1491, 1443, 1387, 1358, 1279, 1254, 1176, 1134, 1075, 969, 926, 843, $759 \mathrm{~cm}^{-1}$; HRMS (ESI) calcd for $\mathrm{C}_{27} \mathrm{H}_{20} \mathrm{~F}_{7} \mathrm{~N}_{2} \mathrm{O}_{4}{ }^{+}[\mathrm{M}+\mathrm{H}]^{+} 569.1306$, found 569.1298. HPLC analysis: 95\% ee (CHIRALPAK IC, isopropanol/hexane $=10: 90,1.0 \mathrm{~mL} / \mathrm{min}, \mathrm{UV}$ : $254 \mathrm{~nm}$ ), $t_{\mathrm{R}}=6.554 \min$ (major), $7.938 \min$ (minor).
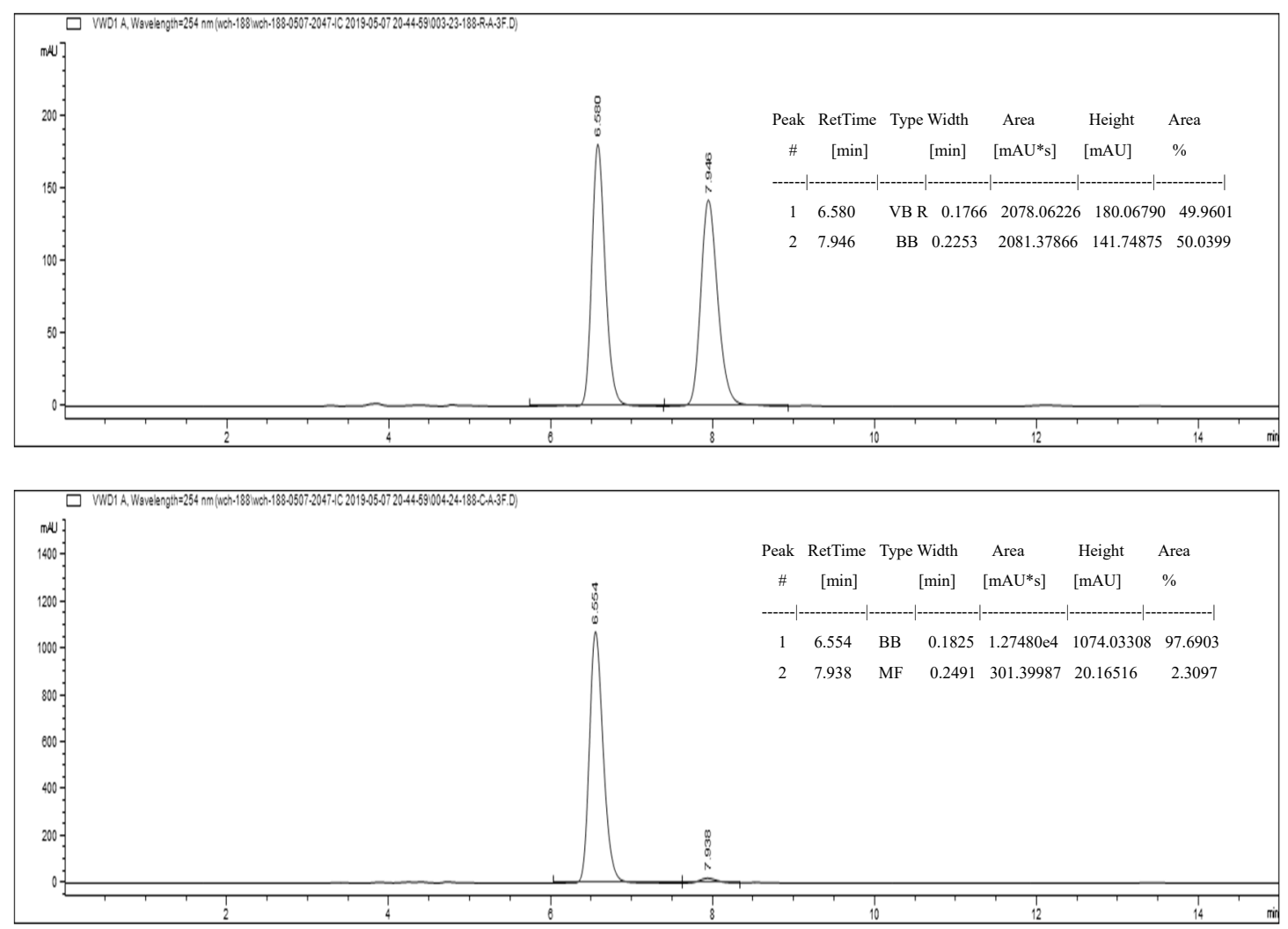
(S)-4-(3,5-bis(trifluoromethyl)benzyl) 1-methyl 3-(4-fluorophenyl)-2-phenyl-2,3-dihydro1H-pyrazole-1,4-dicarboxylate<smiles>CC(=O)N1C=C(C(=O)OCc2cc(C(F)(F)F)cc(C(F)(F)F)c2)C(c2ccc(C(F)(F)F)cc2)N1c1ccccc1</smiles>

Pale yellow solid, 96\% yield (54 mg). Purified by flash chromatography (12\% EtOAc/PE). $\mathrm{mp}=118-120^{\circ} \mathrm{C} ;\left[\alpha{ }^{20}{ }_{\mathrm{D}}=+68.6\left(c 0.63, \mathrm{CH}_{2} \mathrm{Cl}_{2}\right) ;{ }^{1} \mathrm{H}\right.$ NMR $\left(300 \mathrm{MHz}, \mathrm{CDCl}_{3}\right) \delta 8.03(\mathrm{~d}$, $J=1.1 \mathrm{~Hz}, 1 \mathrm{H}), 7.87(\mathrm{~s}, 1 \mathrm{H}), 7.69(\mathrm{~s}, 2 \mathrm{H}), 7.52-7.32(\mathrm{~m}, 4 \mathrm{H}), 7.19-7.00(\mathrm{~m}, 5 \mathrm{H}), 5.46(\mathrm{~s}$, $1 \mathrm{H}), 5.29(\mathrm{~d}, J=13.1 \mathrm{~Hz}, 1 \mathrm{H}), 5.12(\mathrm{~d}, J=13.1 \mathrm{~Hz}, 1 \mathrm{H}), 3.88(\mathrm{~s}, 3 \mathrm{H}) ;{ }^{13} \mathrm{C} \mathrm{NMR}(101 \mathrm{MHz}$, $\left.\mathrm{CDCl}_{3}\right) \delta 162.8(\mathrm{~d}, 1 J \mathrm{C}-\mathrm{F}=247.2 \mathrm{~Hz}), 162.5,151.8,151.2,138.2,136.5(\mathrm{~d}, 4 J \mathrm{C}-\mathrm{F}=2.6 \mathrm{~Hz})$, $136.5,132.0(\mathrm{q}, 2 J \mathrm{C}-\mathrm{F}=33.5 \mathrm{~Hz}), 129.4,128.4(\mathrm{~d}, 3 J \mathrm{C}-\mathrm{F}=8.3 \mathrm{~Hz}), 128.1(\mathrm{q}, 3 J \mathrm{C}-\mathrm{F}=4.3$ $\mathrm{Hz}), 124.3,123.1(\mathrm{q}, 1 J \mathrm{C}-\mathrm{F}=272.8 \mathrm{~Hz}), 122.2(\mathrm{sep}, 3 J \mathrm{C}-\mathrm{F}=3.5 \mathrm{~Hz}), 116.8,115.8(\mathrm{~d}, 2 J \mathrm{C}-\mathrm{F}$ $=21.7 \mathrm{~Hz}$ ), 112.5, 76.4, 64.6, 54.3; IR (film) $v_{\max } 3056,2359,1709$, 1624, 1490, 1446, 1387 , 1357, 1280, 1264, 1178, 1137, 1074, 970, 893, 843, 734, $704 \mathrm{~cm}^{-1}$; HRMS (ESI) calcd for $\mathrm{C}_{27} \mathrm{H}_{20} \mathrm{~F}_{7} \mathrm{~N}_{2} \mathrm{O}_{4}{ }^{+}[\mathrm{M}+\mathrm{H}]^{+}$569.1306, found 569.1296. HPLC analysis: 96\% ee (CHIRALPAK IC, isopropanol/hexane $=10: 90,1.0 \mathrm{~mL} / \mathrm{min}, \mathrm{UV}: 254 \mathrm{~nm}$ ), $t_{\mathrm{R}}=5.972 \mathrm{~min}$ (major), $7.984 \mathrm{~min}$ (minor).
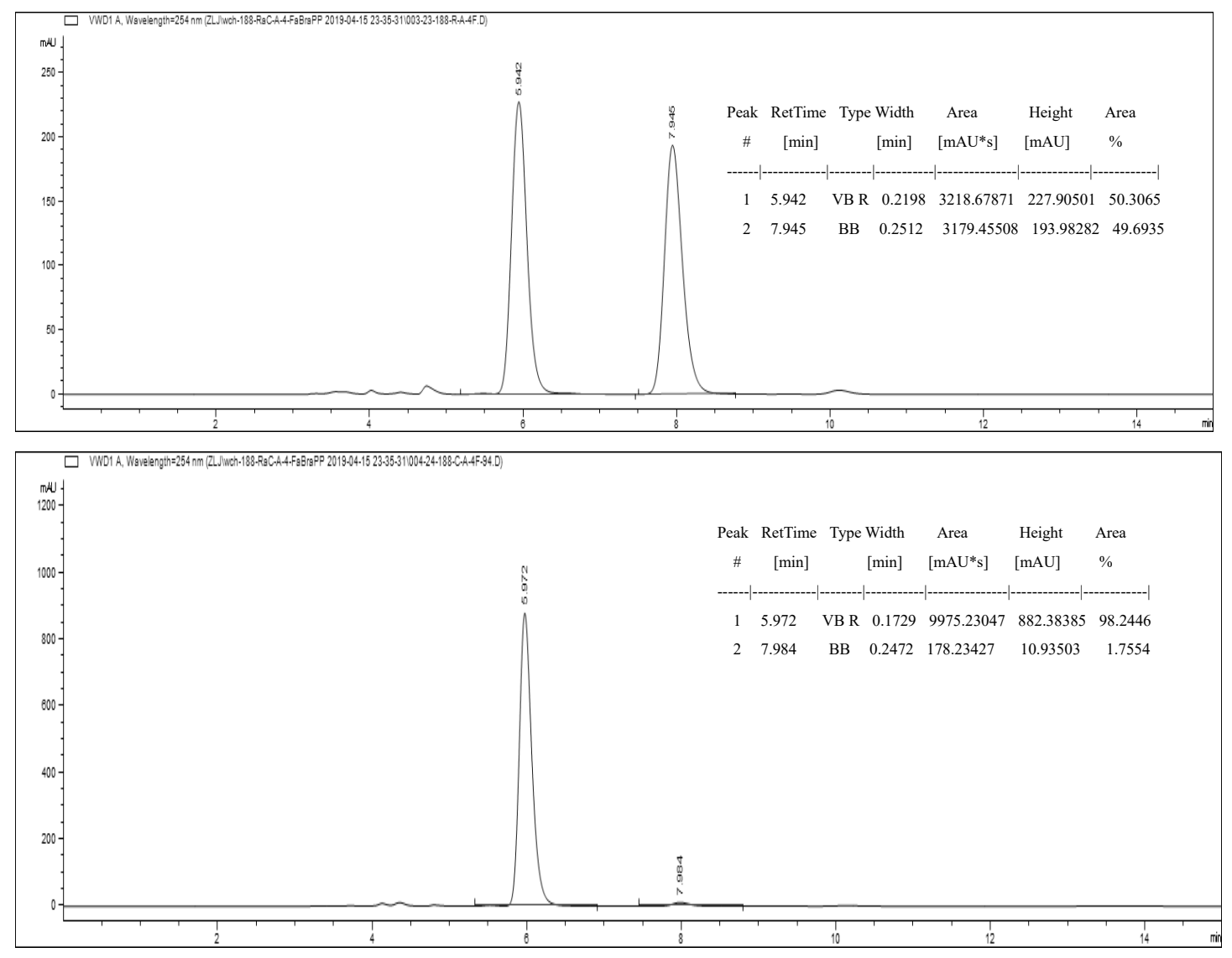


\section{dihydro-1H-pyrazole-1,4-dicarboxylate}<smiles>CC(=O)N1C=C(C(=O)OCc2cc(C(F)(F)F)cc(C(F)(F)F)c2)C(c2cccc(Cl)c2)N1c1ccccc1</smiles>

White solid, 95\% yield $(55 \mathrm{mg})$. Purified by flash chromatography (12\% EtOAc/PE). mp $=$ $159-164{ }^{\circ} \mathrm{C} ;[\alpha]{ }_{\mathrm{D}}^{20}=+129.6\left(c 0.56, \mathrm{CH}_{2} \mathrm{Cl}_{2}\right) ;{ }^{1} \mathrm{H} \mathrm{NMR}\left(300 \mathrm{MHz}, \mathrm{CDCl}_{3}\right) \delta 8.03(\mathrm{~d}, J=$ $1.1 \mathrm{~Hz}, 1 \mathrm{H}), 7.87(\mathrm{~s}, 1 \mathrm{H}), 7.72(\mathrm{~s}, 2 \mathrm{H}), 7.48$ (q, $J=1.5 \mathrm{~Hz}, 1 \mathrm{H}), 7.44-7.31(\mathrm{~m}, 5 \mathrm{H}), 7.22-$ $7.11(\mathrm{~m}, 1 \mathrm{H}), 7.10-6.98(\mathrm{~m}, 2 \mathrm{H}), 5.44(\mathrm{~d}, J=1.1 \mathrm{~Hz}, 1 \mathrm{H}), 5.29$ (d, $J=12.9 \mathrm{~Hz}, 1 \mathrm{H}), 5.13(\mathrm{~d}$, $J=13.0 \mathrm{~Hz}, 1 \mathrm{H}), 3.89(\mathrm{~s}, 3 \mathrm{H}) ;{ }^{13} \mathrm{C}$ NMR $\left(75 \mathrm{MHz}, \mathrm{CDCl}_{3}\right) \delta 162.1,151.4,150.7,142.2$, $137.8,136.4,134.5,131.7(\mathrm{q}, 2 J \mathrm{C}-\mathrm{F}=33.7 \mathrm{~Hz}), 129.8,129.1,128.3,127.9(\mathrm{q}, 3 J \mathrm{C}-\mathrm{F}=3.5$ Hz), 126.6, 124.5, 124.1, $122.8(\mathrm{q}, 1 J \mathrm{C}-\mathrm{F}=270.9 \mathrm{~Hz}) 122.0(\mathrm{sep}, 3 J \mathrm{C}-\mathrm{F}=3.9 \mathrm{~Hz}), 116.6$, 111.7, 76.0, 64.4, 54.0; IR (film) $v_{\max }$ 2960, 2360, 1709, 1624, 1490, 1445, 1387, 1357, 1279, 1256, 1178, 1136, 1112, 1074, 971, 886, 843, 786, $764 \mathrm{~cm}^{-1}$; HRMS (ESI) calcd for $\mathrm{C}_{27} \mathrm{H}_{20} \mathrm{ClF}_{6} \mathrm{~N}_{2} \mathrm{O}_{4}{ }^{+}[\mathrm{M}+\mathrm{H}]^{+}$585.1010, found 585.1003. HPLC analysis: 98\% ee (CHIRALPAK $\mathrm{IC}$, isopropanol $/$ hexane $=10: 90,1.0 \mathrm{~mL} / \mathrm{min}, \mathrm{UV}: 254 \mathrm{~nm}$ ), $t_{\mathrm{R}}=5.937$ min (major), 9.171 $\min (\operatorname{minor})$.
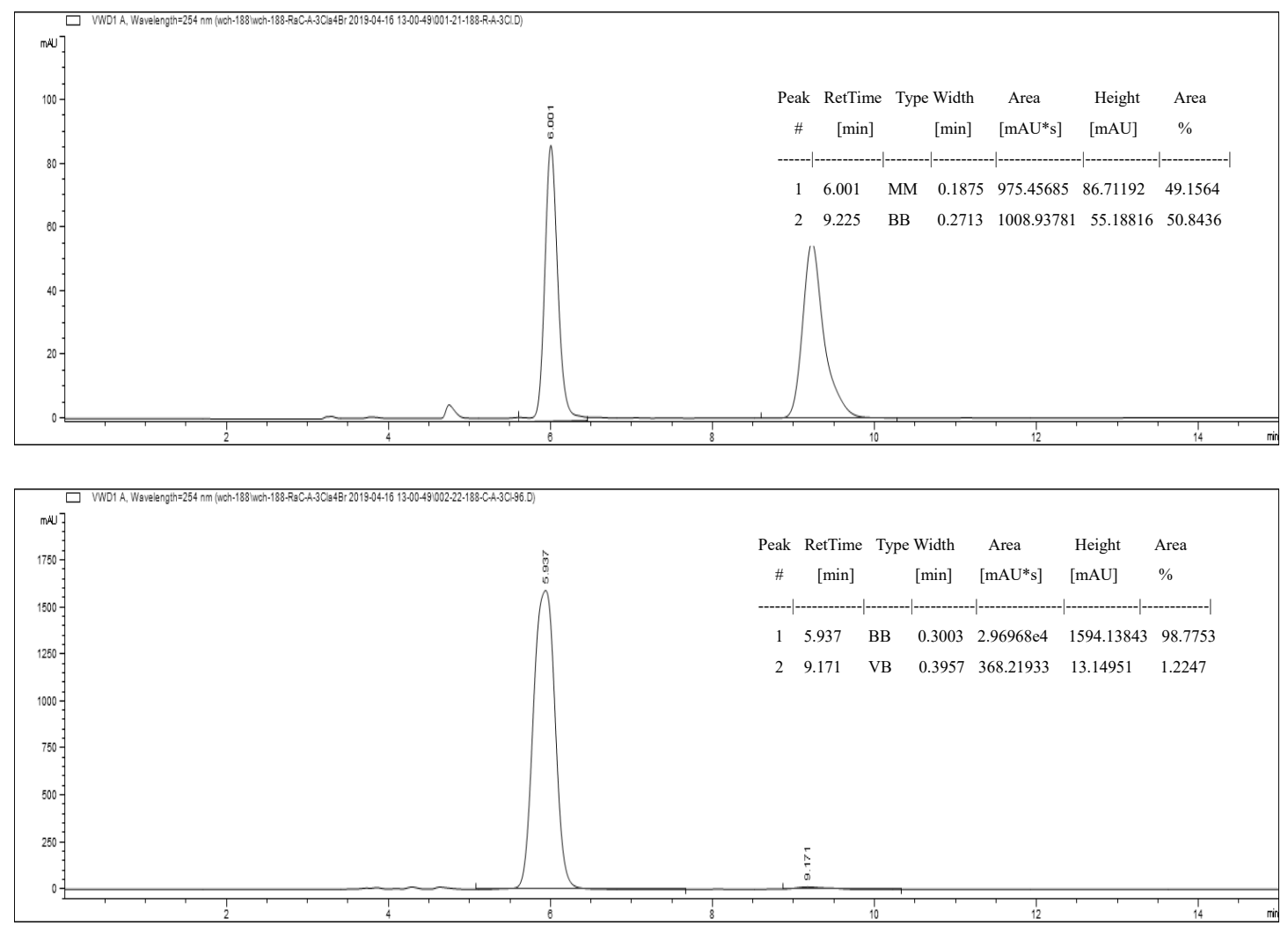
(S)-4-(3,5-bis(trifluoromethyl)benzyl)

1-methyl 3-(4-chlorophenyl)-2-phenyl-2,3-dihydro-1H-pyrazole-1,4-dicarboxylate<smiles>CC(=O)N1C=C(C(=O)OCc2cc(C(F)(F)F)cc(C(F)(F)F)c2)C(c2ccc(Br)cc2)N1c1ccccc1</smiles>

Pale yellow solid, 98\% yield (57 mg). Purified by flash chromatography (12\% EtOAc/PE). $\mathrm{mp}=59-60{ }^{\circ} \mathrm{C} ;[\alpha]^{20}{ }_{\mathrm{D}}=+127.3\left(c 0.48, \mathrm{CH}_{2} \mathrm{Cl}_{2}\right) ;{ }^{1} \mathrm{H}$ NMR $\left(300 \mathrm{MHz}, \mathrm{CDCl}_{3}\right) \delta 8.02(\mathrm{~d}, J$ $=1.0 \mathrm{~Hz}, 1 \mathrm{H}), 7.87(\mathrm{~s}, 1 \mathrm{H}), 7.72(\mathrm{~s}, 2 \mathrm{H}), 7.48-7.33(\mathrm{~m}, 6 \mathrm{H}), 7.15(\mathrm{td}, J=7.2,1.0 \mathrm{~Hz}, 1 \mathrm{H})$, $7.08-6.96(\mathrm{~m}, 2 \mathrm{H}), 5.45(\mathrm{~d}, J=1.1 \mathrm{~Hz}, 1 \mathrm{H}), 5.29(\mathrm{~d}, J=13.0 \mathrm{~Hz}, 1 \mathrm{H}), 5.13(\mathrm{~d}, J=13.1 \mathrm{~Hz}$, 1H), $3.88(\mathrm{~s}, 3 \mathrm{H}) ;{ }^{13} \mathrm{C}$ NMR $\left(101 \mathrm{MHz}, \mathrm{CDCl}_{3}\right) \delta 162.5,151.8,151.1,139.1,138.2,136.6$, 134.3, $132.0(\mathrm{q}, 2 J \mathrm{C}-\mathrm{F}=33.5 \mathrm{~Hz}), 129.4,129.1,128.2(\mathrm{q}, 3 J \mathrm{C}-\mathrm{F}=4.1 \mathrm{~Hz}), 128.1,124.4$, $123.1(\mathrm{q}, 1 J \mathrm{C}-\mathrm{F}=273.8 \mathrm{~Hz}), 122.3(\mathrm{sep}, 3 J \mathrm{C}-\mathrm{F}=4.0 \mathrm{~Hz}), 116.8,112.3,76.3,64.7,54.4$; IR (film) $v_{\max } 3055,2360,1710,1624,1445,1387,1357,1279,1264,1178,1137,1112,1074$, 892, 844, 764, 734, $704 \mathrm{~cm}^{-1}$; HRMS (ESI) calcd for $\mathrm{C}_{27} \mathrm{H}_{20} \mathrm{ClF}_{6} \mathrm{~N}_{2} \mathrm{O}_{4}{ }^{+}[\mathrm{M}+\mathrm{H}]^{+}$585.1010, found 585.0997. HPLC analysis: 97\% ee (CHIRALPAK IC, isopropanol/hexane $=10: 90,1.0$ $\mathrm{mL} / \mathrm{min}, \mathrm{UV}: 254 \mathrm{~nm}$ ), $t_{\mathrm{R}}=5.766 \min$ (major), $7.122 \mathrm{~min}$ (minor).
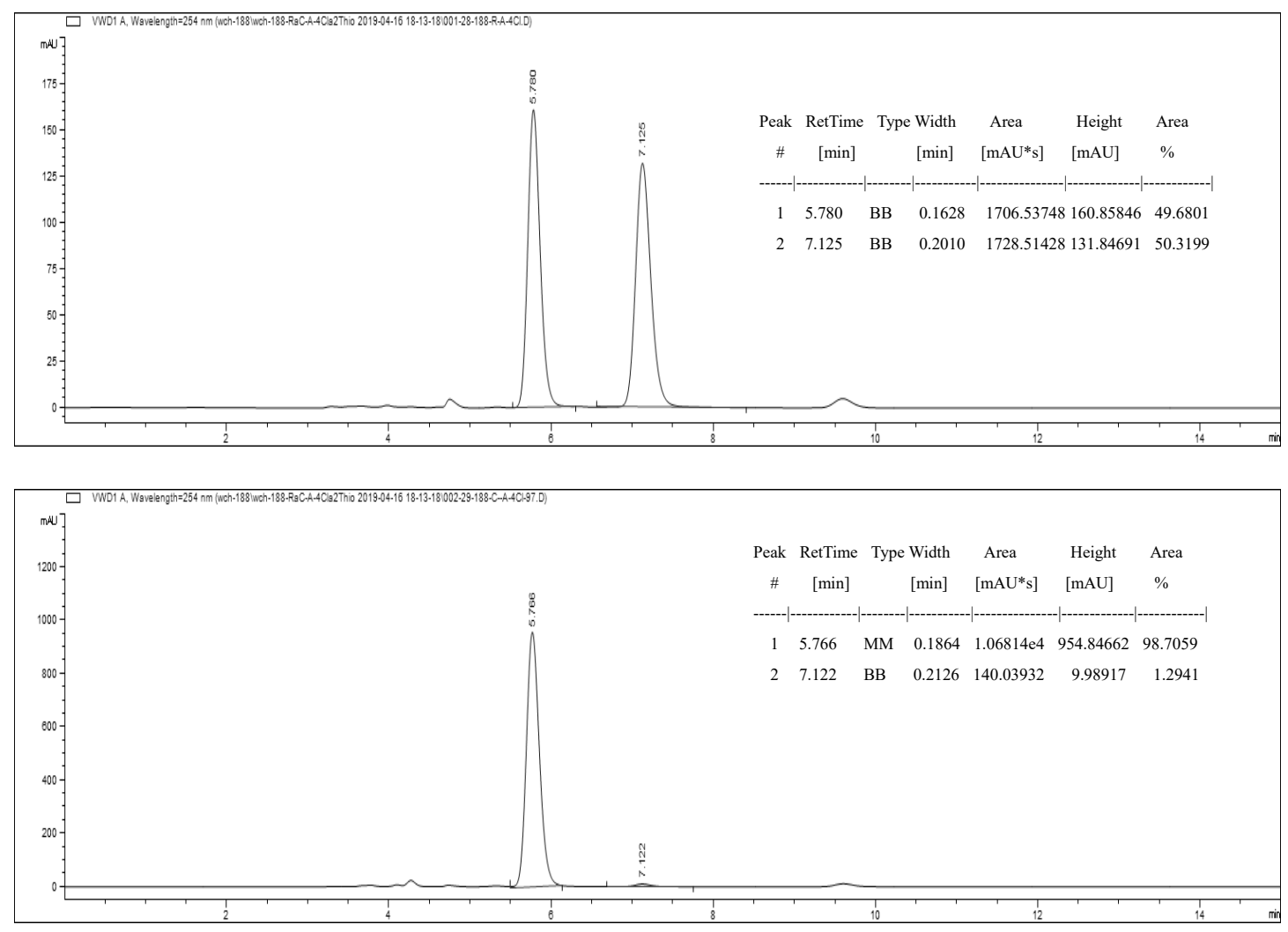


\section{dihydro-1H-pyrazole-1,4-dicarboxylate}<smiles>CC(=O)N1C=C(C(=O)OCc2cc(C(F)(F)F)cc(C(F)(F)F)c2)C(c2ccccc2Br)N1c1ccccc1</smiles>

White solid, 99\% yield (62 mg). Purified by flash chromatography (12\% EtOAc/PE). mp = $145-147{ }^{\circ} \mathrm{C} ;[\alpha]{ }_{\mathrm{D}}^{20}=+100.8\left(c 0.50, \mathrm{CH}_{2} \mathrm{Cl}_{2}\right) ;{ }^{1} \mathrm{H} \mathrm{NMR}\left(300 \mathrm{MHz}, \mathrm{CDCl}_{3}\right) \delta 8.14(\mathrm{~d}, J=$ $1.0 \mathrm{~Hz}, 1 \mathrm{H}), 7.83(\mathrm{~s}, 1 \mathrm{H}), 7.58(\mathrm{~d}, J=8.4 \mathrm{~Hz}, 3 \mathrm{H}), 7.42-7.33$ (m, 3H), $7.32-7.27$ (m, 1H), $7.24-7.09$ (m, 4H), 6.02 (d, $J=1.0 \mathrm{~Hz}, 1 \mathrm{H}), 5.26(\mathrm{~d}, J=12.9 \mathrm{~Hz}, 1 \mathrm{H}), 5.04(\mathrm{~d}, J=12.9 \mathrm{~Hz}$, 1H), $3.87(\mathrm{~s}, 3 \mathrm{H}) ;{ }^{13} \mathrm{C} \mathrm{NMR}\left(101 \mathrm{MHz}, \mathrm{CDCl}_{3}\right) \delta 162.1,152.1,151.1,139.3,138.0,137.4$, 133.1, $131.9(\mathrm{q}, 2 J \mathrm{C}-\mathrm{F}=33.4 \mathrm{~Hz}), 130.1,129.3,129.2,128.6,128.2(\mathrm{q}, 3 J \mathrm{C}-\mathrm{F}=4.0 \mathrm{~Hz})$, 124.4, $123.2(\mathrm{q}, 1 J \mathrm{C}-\mathrm{F}=269.5 \mathrm{~Hz}), 122.5,122.2(\mathrm{sep}, 3 J \mathrm{C}-\mathrm{F}=3.9 \mathrm{~Hz}), 117.4,112.9,75.9$, 64.6, 54.4; IR (film) $v_{\max } 2960,2360,1710,1623,1490,1446,1387,1358,1279,1260,1178$, 1136, 1073, 975, 889, 764, $749 \mathrm{~cm}^{-1}$; HRMS (ESI) calcd for $\mathrm{C}_{27} \mathrm{H}_{20} \mathrm{BrF}_{6} \mathrm{~N}_{2} \mathrm{O}_{4}{ }^{+}[\mathrm{M}+\mathrm{H}]^{+}$ 629.0505, found 629.0483. HPLC analysis: 96\% ee (CHIRALPAK IC, isopropanol/hexane = 10:90, $1.0 \mathrm{~mL} / \mathrm{min}, \mathrm{UV}: 254 \mathrm{~nm}$ ), $t_{\mathrm{R}}=5.868 \mathrm{~min}$ (major), $8.462 \mathrm{~min}$ (minor).
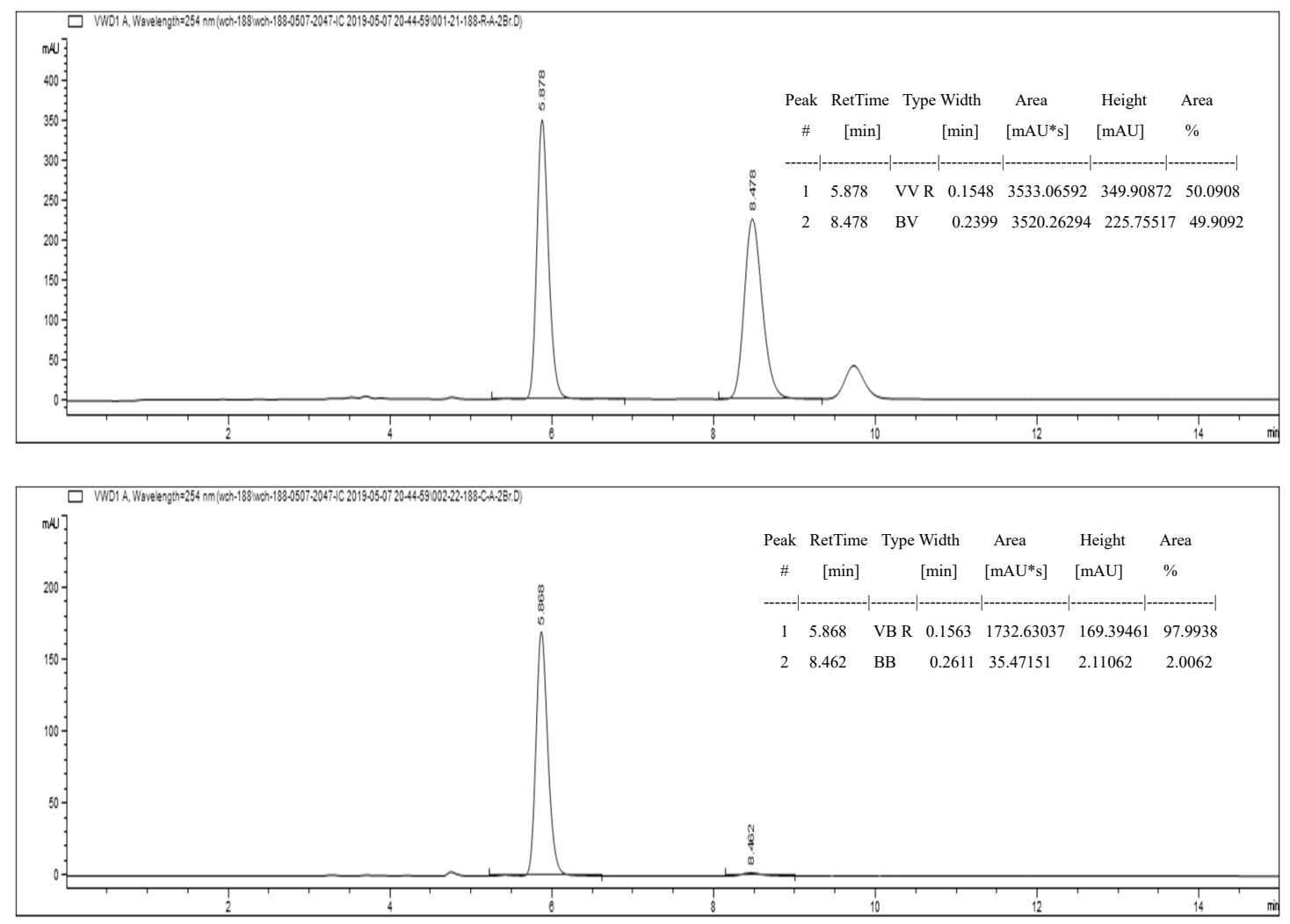

hydro-1H-pyrazole-1,4-dicarboxylate<smiles>CC(=O)N1C=C(C(=O)OCc2cc(C(F)(F)F)cc(C(F)(F)F)c2)C(c2cccc(Br)c2)[C@H]1c1ccccc1</smiles>

White solid, $99 \%$ yield $(62 \mathrm{mg})$. Purified by flash chromatography $(12 \%$ EtOAc/PE). $\mathrm{mp}=$ $165-168{ }^{\circ} \mathrm{C} ;[\alpha]{ }_{\mathrm{D}}^{20}=+128.4\left(c 0.62, \mathrm{CH}_{2} \mathrm{Cl}_{2}\right) ;{ }^{1} \mathrm{H} \mathrm{NMR}\left(300 \mathrm{MHz}, \mathrm{CDCl}_{3}\right) \delta 8.03(\mathrm{~d}, J=$ $1.1 \mathrm{~Hz}, 1 \mathrm{H}), 7.87(\mathrm{~s}, 1 \mathrm{H}), 7.68(\mathrm{dd}, J=26.2,1.8 \mathrm{~Hz}, 3 \mathrm{H}), 7.53-7.25(\mathrm{~m}, 5 \mathrm{H}), 7.20-7.11(\mathrm{~m}$, 1H), $7.09-6.99$ (m, 2H), 5.44 (s, 1H), 5.30 (d, $J=13.0 \mathrm{~Hz}, 1 \mathrm{H}), 5.14$ (d, $J=13.0 \mathrm{~Hz}, 1 \mathrm{H}$ ), $3.89(\mathrm{~s}, 3 \mathrm{H}) ;{ }^{13} \mathrm{C} \mathrm{NMR}\left(75 \mathrm{MHz}, \mathrm{CDCl}_{3}\right) \delta 162.1,151.4,150.7,142.4,137.8,136.4,131.7$ (q, $2 J \mathrm{C}-\mathrm{F}=33.6 \mathrm{~Hz}), 131.2,130.1,129.5,129.1,127.9(\mathrm{q}, 3 J \mathrm{C}-\mathrm{F}=4.0 \mathrm{~Hz}), 124.1,122.8(\mathrm{q}$, $1 J \mathrm{C}-\mathrm{F}=270.9 \mathrm{~Hz}), 122.7,122.0(\mathrm{sep}, 3 J \mathrm{C}-\mathrm{F}=3.8 \mathrm{~Hz}), 116.6,111.7,75.9,64.4,54.0$; IR (film) $v_{\max } 2958,2360,1709,1624,1491,1445,1386,1357,1279,1252,1177,1134,1073$, 971, 885, 843, 785, $764 \mathrm{~cm}^{-1}$; HRMS (ESI) calcd for $\mathrm{C}_{27} \mathrm{H}_{20} \mathrm{BrF}_{6} \mathrm{~N}_{2} \mathrm{O}_{4}{ }^{+}[\mathrm{M}+\mathrm{H}]^{+} 629.0505$, found 629.0500. HPLC analysis: 98\% ee (CHIRALPAK IC, isopropanol/hexane $=10: 90,1.0$ $\mathrm{mL} / \mathrm{min}, \mathrm{UV}: 254 \mathrm{~nm}$ ), $t_{\mathrm{R}}=6.157 \mathrm{~min}$ (major), $9.748 \mathrm{~min}$ (minor).
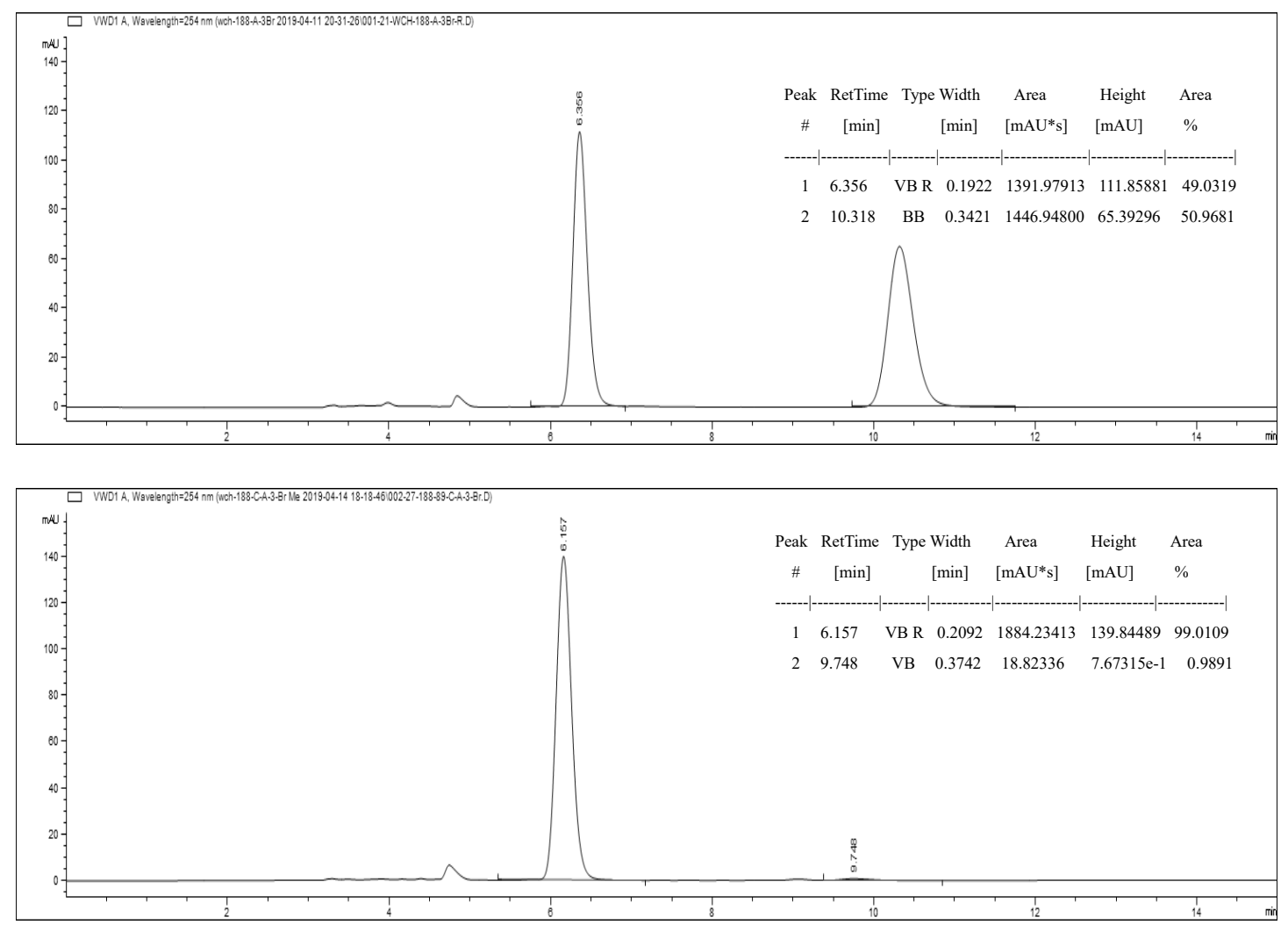
(S)-4-(3,5-bis(trifluoromethyl)benzyl)

1-methyl 3-(4-bromophenyl)-2-phenyl-2,3-dihydro-1H-pyrazole-1,4-dicarboxylate<smiles>CC(=O)N1C=C(C(=O)OCc2cc(C(F)(F)F)cc(C(F)(F)F)c2)[C@H](c2ccc(Br)cc2)N1c1ccccc1</smiles>

Pale yellow solid, 95\% yield (60 mg). Purified by flash chromatography (12\% EtOAc/PE). $\mathrm{mp}=59-60{ }^{\circ} \mathrm{C} ;[\alpha]^{20}{ }_{\mathrm{D}}=+149.5\left(c 0.61, \mathrm{CH}_{2} \mathrm{Cl}_{2}\right) ;{ }^{1} \mathrm{H}$ NMR $\left(300 \mathrm{MHz}, \mathrm{CDCl}_{3}\right) \delta 8.01(\mathrm{~d}, J$ $=1.0 \mathrm{~Hz}, 1 \mathrm{H}), 7.88(\mathrm{~s}, 1 \mathrm{H}), 7.72(\mathrm{~s}, 2 \mathrm{H}), 7.60-7.49(\mathrm{~m}, 2 \mathrm{H}), 7.37(\mathrm{dd}, J=8.9,7.3 \mathrm{~Hz}, 4 \mathrm{H})$, $7.20-7.09(\mathrm{~m}, 1 \mathrm{H}), 7.08-6.97(\mathrm{~m}, 2 \mathrm{H}), 5.44(\mathrm{~s}, 1 \mathrm{H}), 5.28(\mathrm{~d}, J=13.0 \mathrm{~Hz}, 1 \mathrm{H}), 5.13(\mathrm{~d}, J=$ $13.0 \mathrm{~Hz}, 1 \mathrm{H}), 3.88(\mathrm{~s}, 3 \mathrm{H}) ;{ }^{13} \mathrm{C} \mathrm{NMR}\left(75 \mathrm{MHz}, \mathrm{CDCl}_{3}\right) \delta 162.1,151.4,150.8,139.3,137.8$, $136.2,131.71,131.68(\mathrm{q}, 2 J \mathrm{C}-\mathrm{F}=33.5 \mathrm{~Hz}), 129.1,128.0,127.8(\mathrm{q}, 3 J \mathrm{C}-\mathrm{F}=4.0 \mathrm{~Hz}), 124.0$, $122.8(\mathrm{q}, 1 J \mathrm{C}-\mathrm{F}=270.3 \mathrm{~Hz}), 122.1,122.0(\mathrm{sep}, 3 J \mathrm{C}-\mathrm{F}=4.2 \mathrm{~Hz}) 116.5,111.8,76.0,64.3$, 54.0; IR (film) $v_{\max } 3055,2360,1708,1624,1490,1446,1387,1357,1279,1264,1178,1137$, 1073, 970, 895, 844, 764, $734 \mathrm{~cm}^{-1}$; HRMS (ESI) calcd for $\mathrm{C}_{27} \mathrm{H}_{20} \mathrm{BrF}_{6} \mathrm{~N}_{2} \mathrm{O}_{4}{ }^{+}[\mathrm{M}+\mathrm{H}]^{+}$ 629.0505, found 629.0482. HPLC analysis: 98\% ee (CHIRALPAK IC, isopropanol/hexane = 10:90, $1.0 \mathrm{~mL} / \mathrm{min}, \mathrm{UV}: 254 \mathrm{~nm}$ ), $t_{\mathrm{R}}=6.011 \mathrm{~min}$ (major), $7.330 \mathrm{~min}$ (minor).
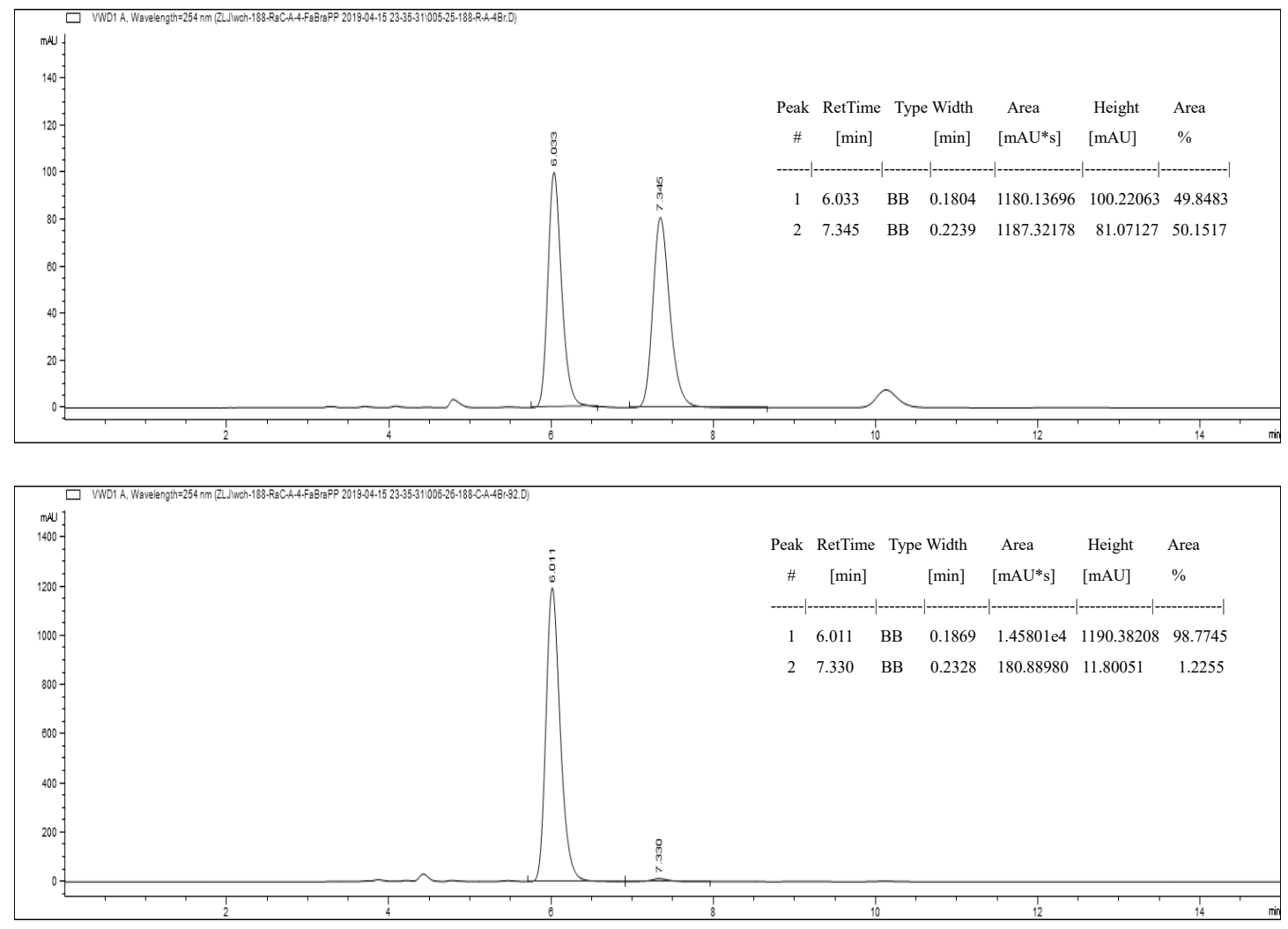
<smiles>CC(=O)N1C=C(C(=O)OCc2cc(C(F)(F)F)cc(C(F)(F)F)c2)[C@H](c2ccc([N+](=O)[O-])cc2)N1c1ccccc1</smiles>

Pale yellow solid, 93\% yield (55 mg). Purified by flash chromatography (12\% EtOAc/PE). $\mathrm{mp}=61-62{ }^{\circ} \mathrm{C} ;[\alpha]^{20}{ }_{\mathrm{D}}=+196.2\left(c 0.55, \mathrm{CH}_{2} \mathrm{Cl}_{2}\right) ;{ }^{1} \mathrm{H}$ NMR $\left(300 \mathrm{MHz}, \mathrm{CDCl}_{3}\right) \delta 8.31-$ $8.21(\mathrm{~m}, 2 \mathrm{H}), 8.01(\mathrm{~d}, J=1.1 \mathrm{~Hz}, 1 \mathrm{H}), 7.86(\mathrm{~s}, 1 \mathrm{H}), 7.76-7.66(\mathrm{~m}, 4 \mathrm{H}), 7.43-7.31(\mathrm{~m}, 2 \mathrm{H})$, $7.21-6.97(\mathrm{~m}, 3 \mathrm{H}), 5.56(\mathrm{~s}, 1 \mathrm{H}), 5.30(\mathrm{~d}, J=13.0 \mathrm{~Hz}, 1 \mathrm{H}), 5.16$ (d, $J=13.0 \mathrm{~Hz}, 1 \mathrm{H}), 3.91(\mathrm{~s}$, $3 \mathrm{H}) ;{ }^{13} \mathrm{C}$ NMR $\left(101 \mathrm{MHz}, \mathrm{CDCl}_{3}\right) \delta 162.4,151.6,150.7,147.8,147.3,138.0,136.9,132.0$ (q, $2 J \mathrm{C}-\mathrm{F}=33.5 \mathrm{~Hz}), 129.6,128.2(\mathrm{q}, 3 J \mathrm{C}-\mathrm{F}=3.8 \mathrm{~Hz}), 127.7,124.7,124.2,123.0(\mathrm{q}, 1 J \mathrm{C}-\mathrm{F}=$ $273.8 \mathrm{~Hz}$ ), $122.4(\mathrm{sep}, 3 J \mathrm{C}-\mathrm{F}=3.8 \mathrm{~Hz}), 117.0,111.5,75.9,64.8,54.5$; IR (film) $v_{\max } 2960$, 2360, 1709, 1623, 1524, 1491, 1445, 1387, 1349, 1279, 1264, 1178, 1136, 1112, 1074, 971, 854, 831, 736, $683 \mathrm{~cm}^{-1}$; HRMS (ESI) calcd for $\mathrm{C}_{27} \mathrm{H}_{18} \mathrm{~F}_{6} \mathrm{~N}_{3} \mathrm{O}_{6}{ }^{+}[\mathrm{M}-\mathrm{H}]^{-}$594.1105, found 594.1111. HPLC analysis: $>99 \%$ ee (CHIRALPAK ID, isopropanol/hexane $=10: 90,1.0$ $\mathrm{mL} / \mathrm{min}, \mathrm{UV}: 254 \mathrm{~nm}$ ), $t_{\mathrm{R}}=13.055 \min$ (major), $16.138 \mathrm{~min}$ (minor).
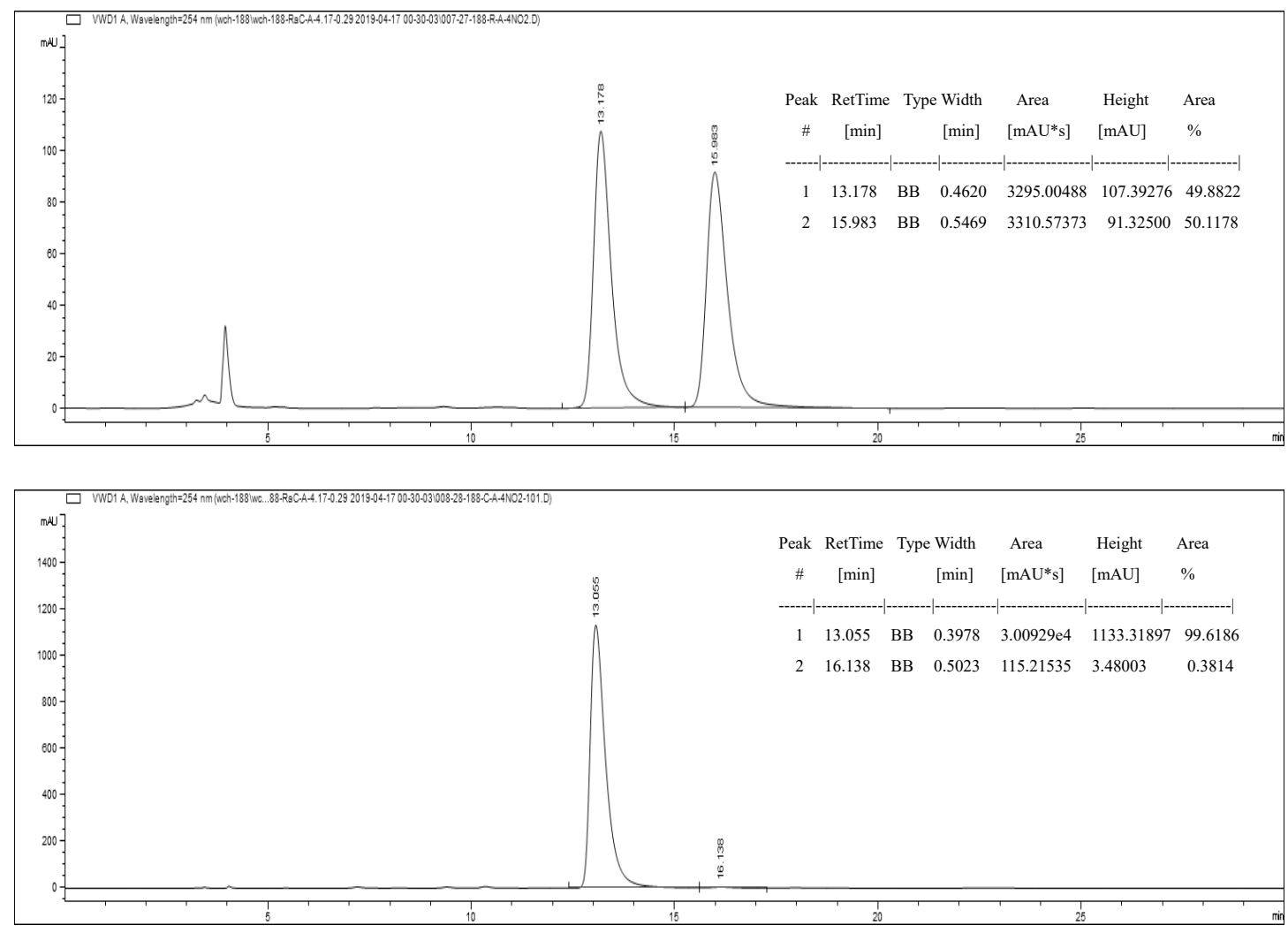


\section{H-pyrazole-1,4-dicarboxylate.}<smiles>CC(=O)N1C=C(C(=O)OCc2cc(C(F)(F)F)cc(C(F)(F)F)c2)C(c2cccc(C)c2)[C@H]1c1ccccc1</smiles>

White solid, $96 \%$ yield $(54 \mathrm{mg})$. Purified by flash chromatography (12\% EtOAc/PE). $\mathrm{mp}=$ $140-144{ }^{\circ} \mathrm{C} ;[\alpha]^{20}{ }_{\mathrm{D}}=+108.7\left(c 0.49, \mathrm{CH}_{2} \mathrm{Cl}_{2}\right) ;{ }^{1} \mathrm{H}$ NMR $\left(300 \mathrm{MHz}, \mathrm{CDCl}_{3}\right) \delta 8.06(\mathrm{~d}, J=$ $1.0 \mathrm{~Hz}, 1 \mathrm{H}), 7.86(\mathrm{~s}, 1 \mathrm{H}), 7.70(\mathrm{~d}, J=1.7 \mathrm{~Hz}, 2 \mathrm{H}), 7.45-7.23(\mathrm{~m}, 6 \mathrm{H}), 7.23-7.04(\mathrm{~m}, 4 \mathrm{H})$, $5.44(\mathrm{~s}, 1 \mathrm{H}), 5.29(\mathrm{~d}, J=13.1 \mathrm{~Hz}, 1 \mathrm{H}), 5.11(\mathrm{~d}, J=13.1 \mathrm{~Hz}, 1 \mathrm{H}), 3.88(\mathrm{~s}, 3 \mathrm{H}), 2.40(\mathrm{~s}, 3 \mathrm{H})$; ${ }^{13} \mathrm{C}$ NMR $\left(75 \mathrm{MHz}, \mathrm{CDCl}_{3}\right) \delta 162.2,151.6,151.2,140.1,138.3,138.0,131.6(\mathrm{q}, 2 J \mathrm{C}-\mathrm{F}=$ $33.5 \mathrm{~Hz}), 136.1,129.0,128.4,127.7(\mathrm{q}, 3 J \mathrm{C}-\mathrm{F}=3.8 \mathrm{~Hz}), 126.9,123.8,123.4,122.8(\mathrm{q}, 1 J \mathrm{C}-\mathrm{F}$ $=270.9 \mathrm{~Hz}), 121.8(\mathrm{sep}, 3 J \mathrm{C}-\mathrm{F}=3.8 \mathrm{~Hz}), 116.5,112.4,76.8,64.2,53.8,21.1$; IR (film) $v_{\max }$ 2959, 2359, 1709, 1624, 1490, 1446, 1387, 1358, 1279, 1264, 1178, 1136, 1074, 970, 890, 843, 764, $735 \mathrm{~cm}^{-1}$; HRMS (ESI) calcd for $\mathrm{C}_{28} \mathrm{H}_{23} \mathrm{~F}_{6} \mathrm{~N}_{2} \mathrm{O}_{4}^{+}[\mathrm{M}+\mathrm{H}]^{+} 565.1557$, found 565.1543 HPLC analysis: 98\% ee (CHIRALPAK IC, isopropanol/hexane $=10: 90,1.0 \mathrm{~mL} / \mathrm{min}, \mathrm{UV}$ : $254 \mathrm{~nm}), t_{\mathrm{R}}=7.481 \min$ (major), $12.059 \min ($ minor).
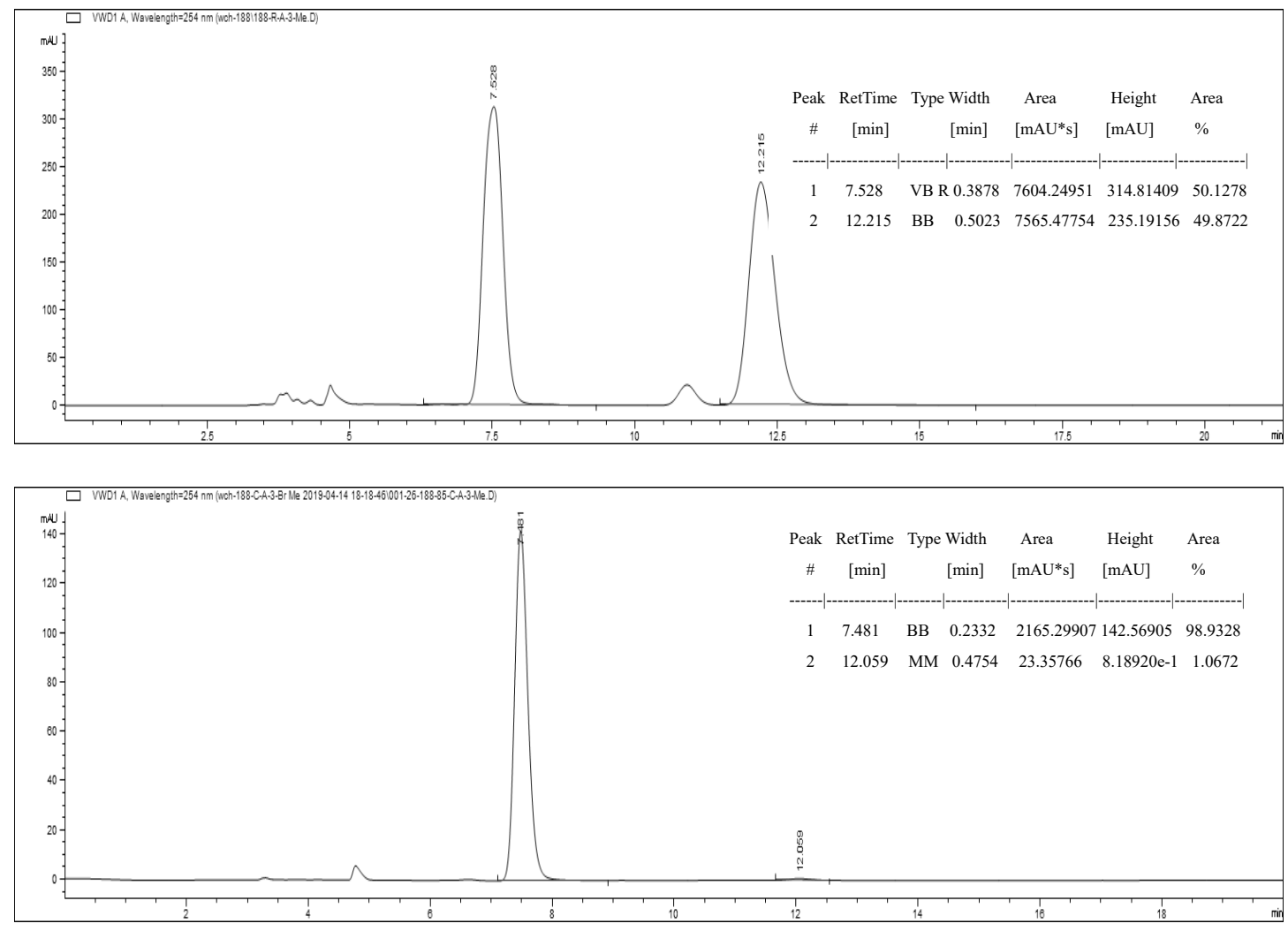
(S)-4-(3,5-bis(trifluoromethyl)benzyl) 1-methyl 2-phenyl-3-(p-tolyl)-2,3-dihydro-1Hpyrazole-1,4-dicarboxylate (3ca).<smiles>CC(=O)N1C=C(C(=O)OCc2cc(C(F)(F)F)cc(C(F)(F)F)c2)[C@H](c2ccc(C)cc2)N1c1ccccc1</smiles>

3la

Pale yellow solid, 95\% yield (54 mg). Purified by flash chromatography (12\% EtOAc/PE). $\mathrm{mp}=49-50{ }^{\circ} \mathrm{C} ;[\alpha]^{20}{ }_{\mathrm{D}}=+121.6\left(c 0.57, \mathrm{CH}_{2} \mathrm{Cl}_{2}\right) ;{ }^{1} \mathrm{H}$ NMR $\left(300 \mathrm{MHz}, \mathrm{CDCl}_{3}\right) \delta 8.04(\mathrm{~d}, J$ $=1.0 \mathrm{~Hz}, 1 \mathrm{H}), 7.85(\mathrm{~s}, 1 \mathrm{H}), 7.67(\mathrm{~d}, J=1.7 \mathrm{~Hz}, 2 \mathrm{H}), 7.41-7.31(\mathrm{~m}, 4 \mathrm{H}), 7.23(\mathrm{~d}, J=7.9 \mathrm{~Hz}$, 2H), $7.18-7.03(\mathrm{~m}, 3 \mathrm{H}), 5.44(\mathrm{~d}, J=1.1 \mathrm{~Hz}, 1 \mathrm{H}), 5.29(\mathrm{~d}, J=13.1 \mathrm{~Hz}, 1 \mathrm{H}), 5.09(\mathrm{~d}, J=13.1$ $\mathrm{Hz}, 1 \mathrm{H}), 3.87$ (s, 3H), 2.40 (s, 3H); ${ }^{13} \mathrm{C}$ NMR (101 MHz, $\left.\mathrm{CDCl}_{3}\right) \delta 162.6,151.5,138.4,138.3$, 137.6, 136.4, 131.9 (q, 2JC-F = 33.6 Hz), 129.6, 129.3, 128.9, 128.0 (q, 3JC-F = 3.8 Hz), 126.6, 124.0, $123.1(\mathrm{sep}, 1 J \mathrm{C}-\mathrm{F}=273.8 \mathrm{~Hz}), 122.2(\mathrm{q}, 3 J \mathrm{C}-\mathrm{F}=3.8 \mathrm{~Hz}), 116.8,112.8,77.0$, 64.5, 54.2, 21.2; IR (film) $v_{\max } 3005,2360,1715,1624,1490,1446,1387,1358,1279,1264$, 1178, 1136, 1074, 891, 764, 748, 734, $704 \mathrm{~cm}^{-1}$; HRMS (ESI) calcd for $\mathrm{C}_{28} \mathrm{H}_{23} \mathrm{~F}_{6} \mathrm{~N}_{2} \mathrm{O}_{4}{ }^{+}$ $[\mathrm{M}+\mathrm{H}]^{+}$565.1557, found 565.1544. HPLC analysis: 98\% ee (CHIRALPAK ID, isopropanol/hexane $=10: 90,1.0 \mathrm{~mL} / \mathrm{min}, \mathrm{UV}: 254 \mathrm{~nm}$ ), $t_{\mathrm{R}}=7.502 \mathrm{~min}$ (major), $9.979 \mathrm{~min}$ (minor).
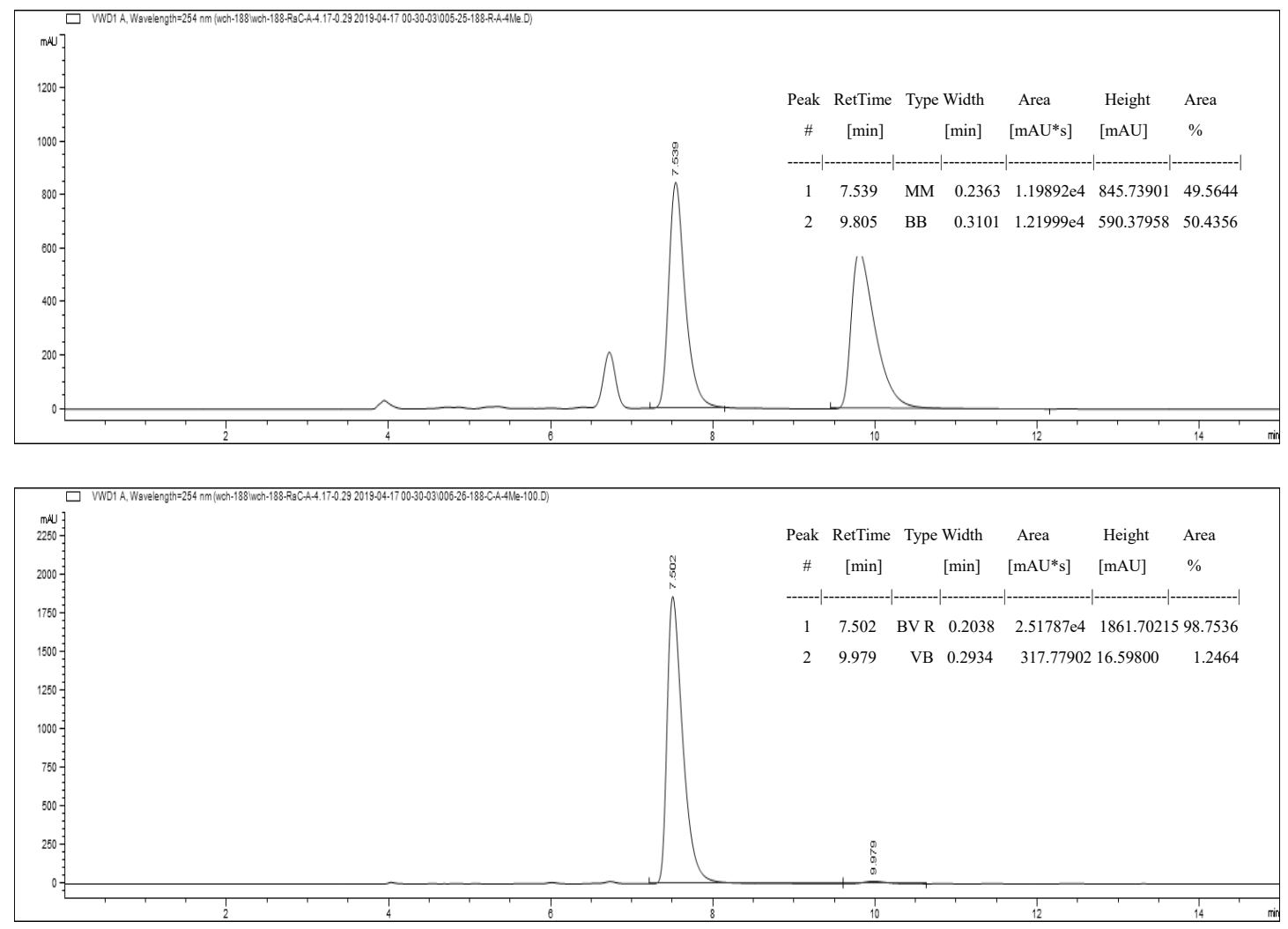
(S)-4-(3,5-bis(trifluoromethyl)benzyl) 1-methyl 3-(2-methoxyphenyl)-2-phenyl-2,3dihydro-1H-pyrazole-1,4-dicarboxylate<smiles>COc1ccccc1[C@H]1C(C(=O)OCc2cc(C(F)(F)F)cc(C(F)(F)F)c2)=CN(C(C)=O)N1c1ccccc1</smiles>

Pale yellow solid, 93\% yield (54 mg). Purified by flash chromatography (12\% EtOAc/PE). $\mathrm{mp}=108-120{ }^{\circ} \mathrm{C} ;[\alpha]^{20}{ }_{\mathrm{D}}=+50.7\left(c 0.38, \mathrm{CH}_{2} \mathrm{Cl}_{2}\right) ;{ }^{1} \mathrm{H}$ NMR $\left(300 \mathrm{MHz}, \mathrm{CDCl}_{3}\right) \delta 8.11(\mathrm{~s}$, 1H), $7.84(\mathrm{~s}, 1 \mathrm{H}), 7.62(\mathrm{~s}, 2 \mathrm{H}), 7.45-7.22(\mathrm{~m}, 4 \mathrm{H}), 7.21-7.05(\mathrm{~m}, 3 \mathrm{H}), 6.99$ (t, J=8.1 Hz, 2H), $5.79(\mathrm{~d}, J=1.1 \mathrm{~Hz}, 1 \mathrm{H}), 5.25(\mathrm{~d}, J=13.2 \mathrm{~Hz}, 1 \mathrm{H}), 5.05(\mathrm{~d}, J=13.2 \mathrm{~Hz}, 1 \mathrm{H}), 3.86(\mathrm{~s}$, 3H), $3.85(\mathrm{~s}, 3 \mathrm{H}) ;{ }^{13} \mathrm{C} \mathrm{NMR}\left(101 \mathrm{MHz}, \mathrm{CDCl}_{3}\right) \delta 162.5,157.5,153.1,151.8,138.4,137.1$, $131.8(\mathrm{q}, 2 J \mathrm{C}-\mathrm{F}=33.4 \mathrm{~Hz}), 130.0,129.1,128.6,127.9(\mathrm{q}, 3 J \mathrm{C}-\mathrm{F}=3.8 \mathrm{~Hz}), 127.4,123.5$, $123.1(\mathrm{q}, 1 J \mathrm{C}-\mathrm{F}=273.9 \mathrm{~Hz}), 122.1(\mathrm{sep}, 3 J \mathrm{C}-\mathrm{F}=3.9 \mathrm{~Hz}), 120.8,116.6,111.7,111.5$, 73.4, 64.4, 55.3, 54.1; IR (film) $v_{\max } 2958,2359,1712,1624,1491,1445,1387,1358,1279,1264$, 1177, 1135, 1075, 971, 887, 844, $750 \mathrm{~cm}^{-1}$; HRMS (ESI) calcd for $\mathrm{C}_{28} \mathrm{H}_{23} \mathrm{~F}_{6} \mathrm{~N}_{2} \mathrm{O}_{5}{ }^{+}[\mathrm{M}+\mathrm{H}]^{+}$ 581.1506, found 581.1490. HPLC analysis: 94\% ee (CHIRALPAK IC, isopropanol/hexane = 10:90, $1.0 \mathrm{~mL} / \mathrm{min}, \mathrm{UV}: 254 \mathrm{~nm}$ ), $t_{\mathrm{R}}=9.200 \mathrm{~min}$ (major), $16.886 \mathrm{~min}$ (minor).
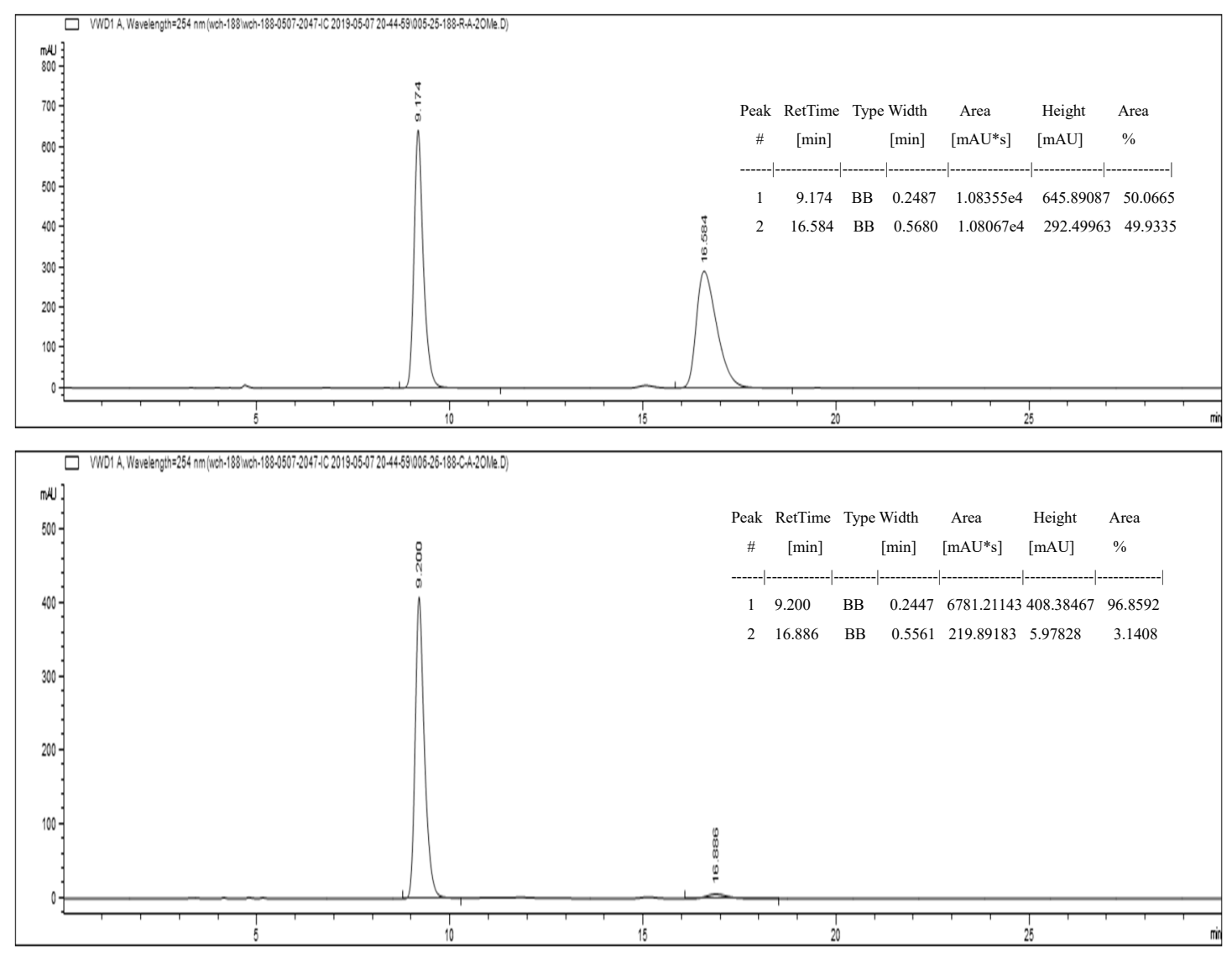
(S)-4-(3,5-bis(trifluoromethyl)benzyl) 1-methyl 3-(3-methoxyphenyl)-2-phenyl-2,3-dihydro-1H-pyrazole-1,4-dicarboxylate<smiles>COc1cccc([C@H]2C(C(=O)OCc3cc([14CH3])cc(C(F)(F)F)c3)=CN(C(C)=O)N2c2ccccc2)c1</smiles>

White solid, 96\% yield $(56 \mathrm{mg})$. Purified by flash chromatography $(12 \%$ EtOAc/PE). mp $=$ $120-122{ }^{\circ} \mathrm{C} ;[\alpha]{ }^{20}{ }_{\mathrm{D}}=+115.7\left(c 0.52, \mathrm{CH}_{2} \mathrm{Cl}_{2}\right) ;{ }^{1} \mathrm{H}$ NMR $\left(300 \mathrm{MHz}, \mathrm{CDCl}_{3}\right) \delta 8.03(\mathrm{~d}, J=$ $1.0 \mathrm{~Hz}, 1 \mathrm{H}), 7.93-7.81(\mathrm{~m}, 1 \mathrm{H}), 7.70(\mathrm{~d}, J=1.7 \mathrm{~Hz}, 2 \mathrm{H}), 7.36(\mathrm{dt}, J=8.2,7.3 \mathrm{~Hz}, 3 \mathrm{H}), 7.19$ $-7.02(\mathrm{~m}, 5 \mathrm{H}), 6.91(\mathrm{ddd}, J=8.3,2.6,0.9 \mathrm{~Hz}, 1 \mathrm{H}), 5.46(\mathrm{~d}, J=1.1 \mathrm{~Hz}, 1 \mathrm{H}), 5.29(\mathrm{~d}, J=13.1$ $\mathrm{Hz}, 1 \mathrm{H}), 5.12(\mathrm{~d}, J=13.1 \mathrm{~Hz}, 1 \mathrm{H}), 3.87(\mathrm{~s}, 3 \mathrm{H}), 3.83(\mathrm{~s}, 3 \mathrm{H}) ;{ }^{13} \mathrm{C} \mathrm{NMR}\left(101 \mathrm{MHz}, \mathrm{CDCl}_{3}\right) \delta$ 162.6, 160.1, 151.9, 151.3, 142.0, 138.3, 136.5, 131.9 (q, 2JC-F = 33.5 Hz), 130.0, 129.4, $128.2(\mathrm{q}, 3 J \mathrm{C}-\mathrm{F}=4.1 \mathrm{~Hz}), 124.1,123.1(\mathrm{q}, 1 J \mathrm{C}-\mathrm{F}=273.8 \mathrm{~Hz}), 122.2(\mathrm{sep}, 3 J \mathrm{C}-\mathrm{F}=4.2 \mathrm{~Hz})$, 118.9, 116.8, 113.4, 112.7, 112.6, 76.8, 64.6, 55.2, 54.3; IR (film) $v_{\max } 2959,2358,1710,1624$, 1490, 1445, 1387, 1358, 1279, 1258, 1177, 1135, 1074, 971, 886, 843, 765, $750 \mathrm{~cm}^{-1}$; HRMS (ESI) calcd for $\mathrm{C}_{28} \mathrm{H}_{23} \mathrm{~F}_{6} \mathrm{~N}_{2} \mathrm{O}_{5}{ }^{+}[\mathrm{M}+\mathrm{H}]^{+} 581.1506$, found 581.1494. HPLC analysis: $98 \%$ ee $($ CHIRALPAK IC, isopropanol $/$ hexane $=10: 90,1.0 \mathrm{~mL} / \mathrm{min}, \mathrm{UV}: 254 \mathrm{~nm}), t_{\mathrm{R}}=11.381 \mathrm{~min}$ (major), $25.451 \mathrm{~min}$ (minor).
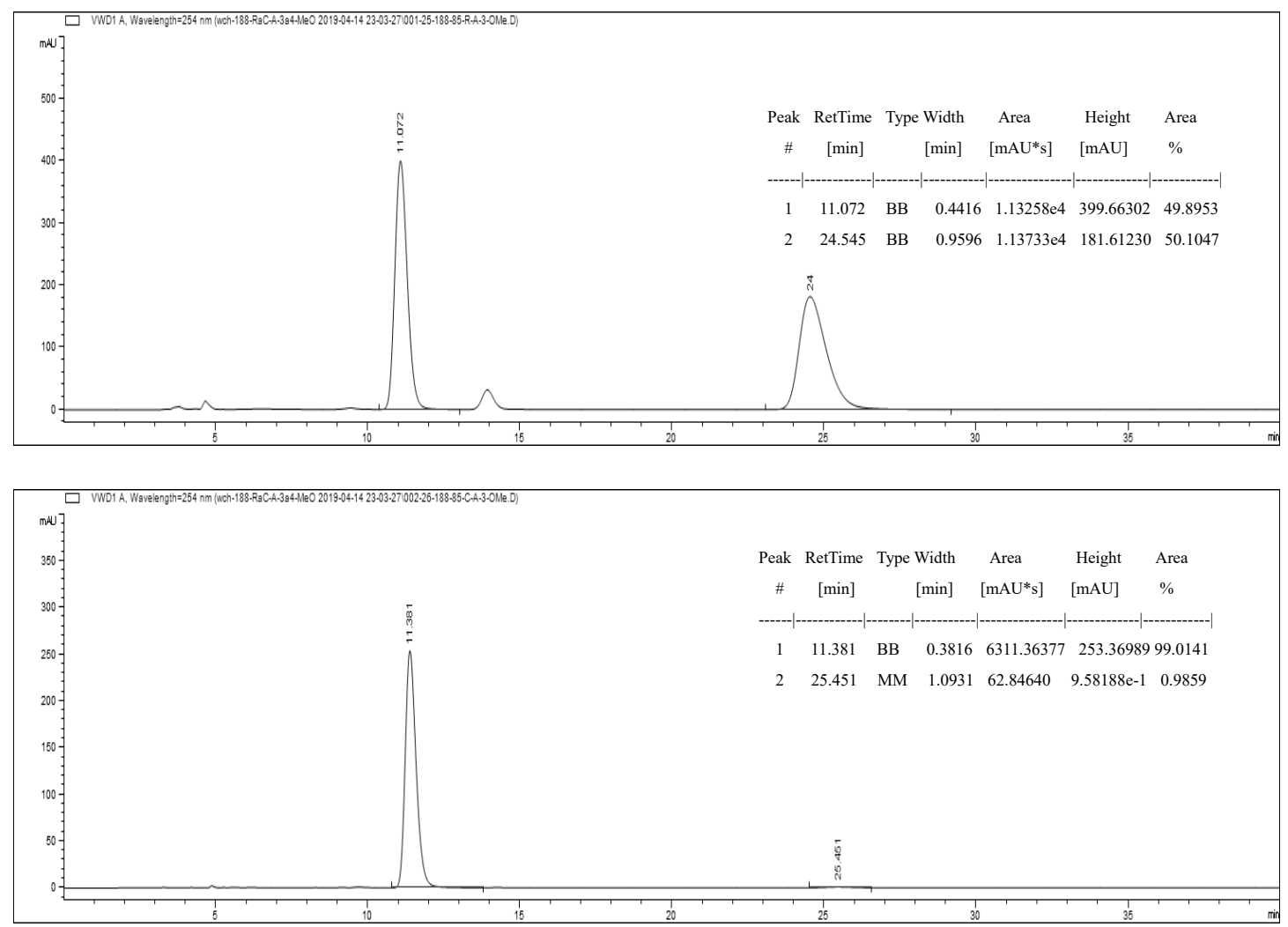
(S)-4-(3,5-bis(trifluoromethyl)benzyl) 1-methyl 3-(4-methoxyphenyl)-2-phenyl-2,3-dihydro-1H-pyrazole-1,4-dicarboxylate<smiles>COc1ccc([C@H]2C(C(=O)OCc3cc(OC)cc(C(F)(F)F)c3)=CN(C(C)=O)N2c2ccccc2)cc1</smiles>

Pale yellow solid, 99\% yield (58 mg). Purified by flash chromatography (12\% EtOAc/PE). $\mathrm{mp}=52-53{ }^{\circ} \mathrm{C} ;[\alpha]^{20}{ }_{\mathrm{D}}=+130.4\left(c 0.62, \mathrm{CH}_{2} \mathrm{Cl}_{2}\right) ;{ }^{1} \mathrm{H}$ NMR $\left(400 \mathrm{MHz}, \mathrm{CDCl}_{3}\right) \delta 8.03(\mathrm{~d}, J$ $=1.1 \mathrm{~Hz}, 1 \mathrm{H}), 7.85(\mathrm{~s}, 1 \mathrm{H}), 7.71-7.65(\mathrm{~m}, 2 \mathrm{H}), 7.41-7.32(\mathrm{~m}, 4 \mathrm{H}), 7.18-6.91(\mathrm{~m}, 5 \mathrm{H})$, $5.42(\mathrm{~d}, J=1.1 \mathrm{~Hz}, 1 \mathrm{H}), 5.27(\mathrm{~d}, J=13.2 \mathrm{~Hz}, 1 \mathrm{H}), 5.10(\mathrm{~d}, J=13.1 \mathrm{~Hz}, 1 \mathrm{H}), 3.86(\mathrm{~s}, 3 \mathrm{H})$, $3.84(\mathrm{~s}, 3 \mathrm{H}) ;{ }^{13} \mathrm{C} \mathrm{NMR}\left(101 \mathrm{MHz}, \mathrm{CDCl}_{3}\right) \delta 162.6,159.8,152.0,151.4,138.3,136.3,132.7$, $131.9(\mathrm{q}, 2 J \mathrm{C}-\mathrm{F}=33.5 \mathrm{~Hz}), 129.3,128.0(\mathrm{q}, 3 J \mathrm{C}-\mathrm{F}=3.4 \mathrm{~Hz}), 127.9,124.1,123.1(\mathrm{q}, 1 J \mathrm{C}-\mathrm{F}=$ $273.7 \mathrm{~Hz}), 122.2(\mathrm{sep}, 3 J \mathrm{C}-\mathrm{F}=3.4 \mathrm{~Hz}), 116.8,114.3,112.8,76.8,64.6,55.2,54.3$; IR (film) $v_{\max } 2959,2361,1709,1623,1490,1445,1387,1357,1279,1264,1175,1135,1074,969,887$, 843, 785, 765, $735 \mathrm{~cm}^{-1}$; HRMS (ESI) calcd for $\mathrm{C}_{28} \mathrm{H}_{23} \mathrm{~F}_{6} \mathrm{~N}_{2} \mathrm{O}_{5}^{+}[\mathrm{M}+\mathrm{H}]^{+}$581.1506, found 581.1494. HPLC analysis: 96\% ee (CHIRALPAK IC, isopropanol/hexane $=10: 90,1.0$ $\mathrm{mL} / \mathrm{min}, \mathrm{UV}: 254 \mathrm{~nm}$ ), $t_{\mathrm{R}}=11.393 \mathrm{~min}$ (major), $16.238 \mathrm{~min}$ (minor).
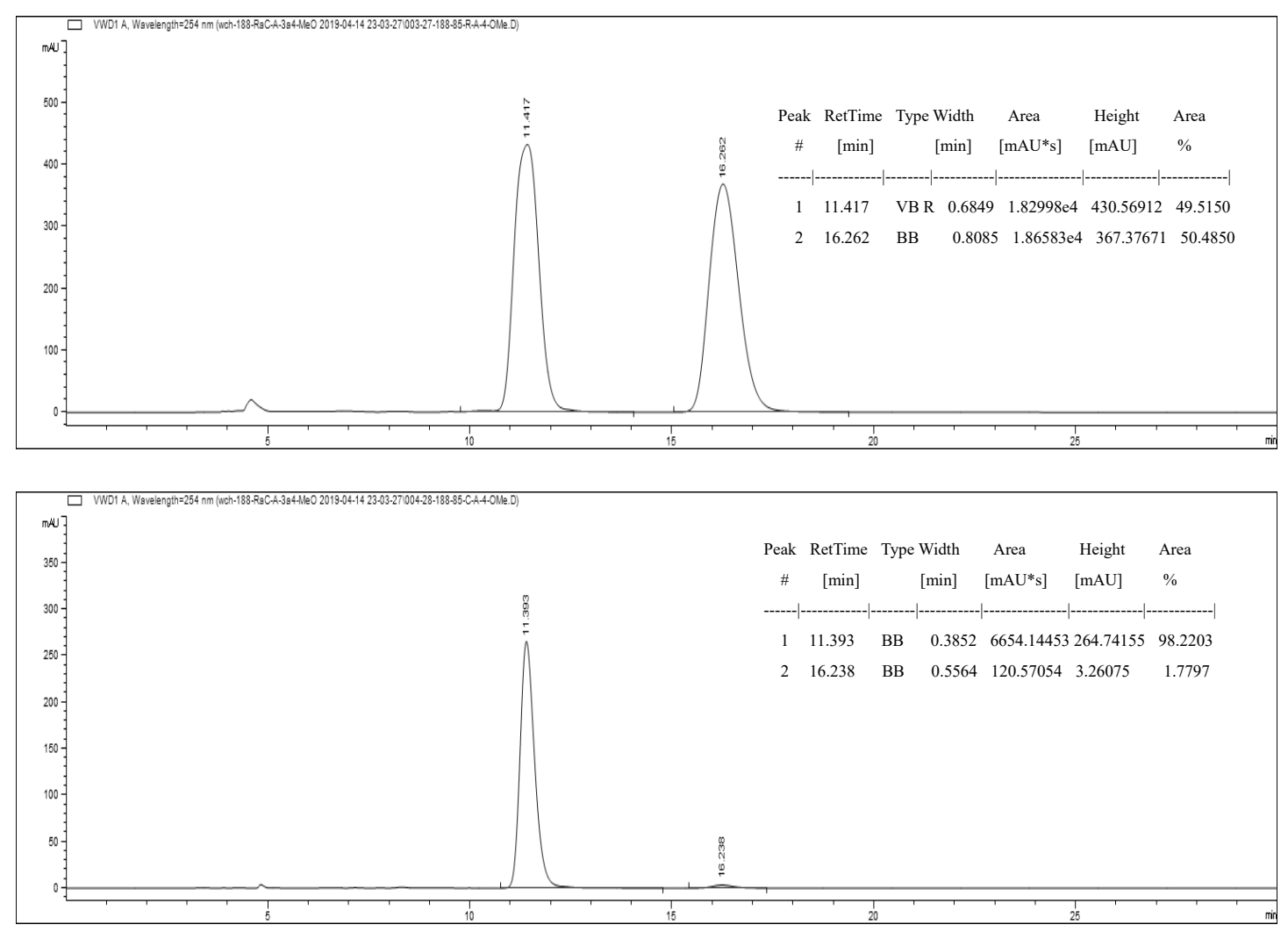
(S)-4-(3,5-bis(trifluoromethyl)benzyl) 1-methyl 3-(3,4-dimethoxyphenyl)-2-phenyl-2,3dihydro-1H-pyrazole-1,4-dicarboxylate<smiles>COc1ccc([C@H]2C(C(=O)OCc3cc(C(F)(F)F)cc(C(F)(F)F)c3)=CN(C(C)=O)N2c2ccccc2)cc1OC</smiles>

Pale yellow solid, 98\% yield (60 mg). Purified by flash chromatography (12\% EtOAc/PE). $\mathrm{mp}=49-50^{\circ} \mathrm{C} ;[\alpha]^{20}{ }_{\mathrm{D}}=+121.6\left(c 0.57, \mathrm{CH}_{2} \mathrm{Cl}_{2}\right) ;{ }^{1} \mathrm{H} \mathrm{NMR}\left(300 \mathrm{MHz}, \mathrm{CDCl}_{3}\right) \delta 7.99(\mathrm{~d}, J=$ $1.0 \mathrm{~Hz}, 1 \mathrm{H}), 7.85(\mathrm{~s}, 1 \mathrm{H}), 7.71(\mathrm{~s}, 2 \mathrm{H}), 7.42-7.31(\mathrm{~m}, 2 \mathrm{H}), 7.18-6.98(\mathrm{~m}, 5 \mathrm{H}), 6.90(\mathrm{~d}, J=$ $8.2 \mathrm{~Hz}, 1 \mathrm{H}), 5.43$ (d, $J=1.0 \mathrm{~Hz}, 1 \mathrm{H}), 5.27$ (d, $J=13.1 \mathrm{~Hz}, 1 \mathrm{H}), 5.14(\mathrm{~d}, J=13.1 \mathrm{~Hz}, 1 \mathrm{H})$, 3.91 (s, 3H), 3.88 (s, 3H), 3.86 (s, 3H); ${ }^{13} \mathrm{C}$ NMR (101 MHz, $\left.\mathrm{CDCl}_{3}\right) \delta 162.7,152.1,151.3$, $149.4,149.2,138.3,136.3,133.0,132.0(\mathrm{q}, 2 J \mathrm{C}-\mathrm{F}=33.5 \mathrm{~Hz}), 129.3,128.1(\mathrm{q}, 3 J \mathrm{C}-\mathrm{F}=3.6$ $\mathrm{Hz}), 124.1,123.1(\mathrm{q}, 1 J \mathrm{C}-\mathrm{F}=273.8 \mathrm{~Hz}), 122.2(\mathrm{sep}, 3 J \mathrm{C}-\mathrm{F}=3.8 \mathrm{~Hz}), 118.8,116.7,112.9$, 111.2, 109.6, 76.6, 64.6, 55.9, 55.8, 54.3; IR (film) $v_{\max } 2960,2360,1709,1623,1524,1491$, 1387, 1349, 1279, 1264, 1178, 1136, 1112, 1074, 971, 854, 831, 736, $683 \mathrm{~cm}^{-1}$; HRMS (ESI) calcd for $\mathrm{C}_{29} \mathrm{H}_{25} \mathrm{~F}_{6} \mathrm{~N}_{2} \mathrm{O}_{6}{ }^{+}[\mathrm{M}+\mathrm{H}]^{+}$611.1611, found 611.1601. HPLC analysis: $99 \%$ ee $($ CHIRALPAK IC, isopropanol $/$ hexane $=10: 90,1.0 \mathrm{~mL} / \mathrm{min}, \mathrm{UV}: 254 \mathrm{~nm}), t_{\mathrm{R}}=19.959 \mathrm{~min}$ (major), 37.615 min (minor).
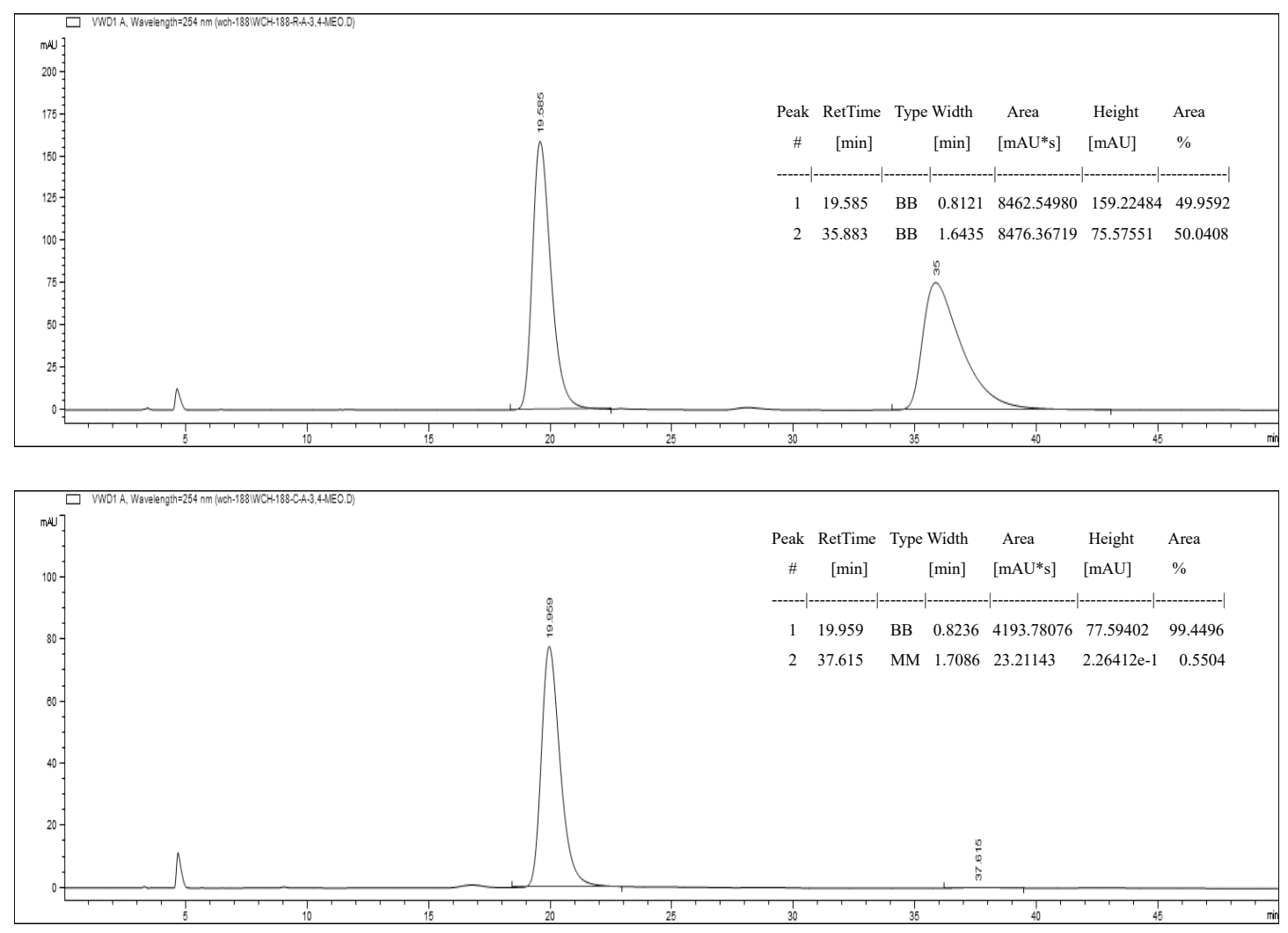
(S)-4-(3,5-bis(trifluoromethyl)benzyl)

1-methyl 3-(naphthalen-2-yl)-2-phenyl-2,3-dihydro-1H-pyrazole-1,4-dicarboxylate<smiles>CC(=O)N1C=C(C(=O)OCc2cc(C(F)(F)F)cc(C(F)(F)F)c2)[C@H](c2ccc3ccccc3c2)N1c1ccccc1</smiles>

Pale yellow solid, 98\% yield (59 mg). Purified by flash chromatography (12\% EtOAc/PE). $\mathrm{mp}=62-63{ }^{\circ} \mathrm{C} ;[\alpha]^{20}{ }_{\mathrm{D}}=+171.8\left(c 0.60, \mathrm{CH}_{2} \mathrm{Cl}_{2}\right) ;{ }^{1} \mathrm{H}$ NMR $\left(300 \mathrm{MHz}, \mathrm{CDCl}_{3}\right) \delta 8.09(\mathrm{~d}, J$ $=1.1 \mathrm{~Hz}, 1 \mathrm{H}), 7.98-7.81(\mathrm{~m}, 5 \mathrm{H}), 7.70(\mathrm{~d}, J=1.7 \mathrm{~Hz}, 2 \mathrm{H}), 7.65-7.48(\mathrm{~m}, 3 \mathrm{H}), 7.44-7.35$ $(\mathrm{m}, 2 \mathrm{H}), 7.21-7.08(\mathrm{~m}, 3 \mathrm{H}), 5.66(\mathrm{~d}, J=1.1 \mathrm{~Hz}, 1 \mathrm{H}), 5.25(\mathrm{~d}, J=13.0 \mathrm{~Hz}, 1 \mathrm{H}), 5.11(\mathrm{~d}, J=$ $13.0 \mathrm{~Hz}, 1 \mathrm{H}), 3.91(\mathrm{~s}, 3 \mathrm{H}) ;{ }^{13} \mathrm{C} \mathrm{NMR}\left(101 \mathrm{MHz}, \mathrm{CDCl}_{3}\right) \delta 162.6,152.0,151.4,138.2,137.8$, 136.5, 133.37, 133.35, 131.9 (q, 2JC-F = 33.6 Hz), 129.4, 129.0, 129.00, 128.2, 127.8, 126.4, 126.3, 125.7, 124.4, 124.2, $123.1(\mathrm{q}, 1 J \mathrm{C}-\mathrm{F}=273.8 \mathrm{~Hz}), 122.2(\mathrm{sep}, 1 J \mathrm{C}-\mathrm{F}=3.4 \mathrm{~Hz}), 116.9$, 112.6, 77.2, 64.6, 54.3; IR (film) $v_{\max } 3057,2359,1709$, 1624, 1490, 1445, 1387, 1357, 1279, 1264, 1177, 1136, 1073, 893, 764, 734, $704 \mathrm{~cm}^{-1}$; HRMS (ESI) calcd for $\mathrm{C}_{31} \mathrm{H}_{23} \mathrm{~F}_{6} \mathrm{~N}_{2} \mathrm{O}_{4}{ }^{+}$ $[\mathrm{M}+\mathrm{H}]^{+}$601.1557, found 601.1545. HPLC analysis: 99\% ee (CHIRALPAK IC, isopropanol/hexane $=10: 90,1.0 \mathrm{~mL} / \mathrm{min}, \mathrm{UV}: 254 \mathrm{~nm}$ ), $t_{\mathrm{R}}=7.982 \mathrm{~min}$ (major), $10.004 \mathrm{~min}$ (minor).
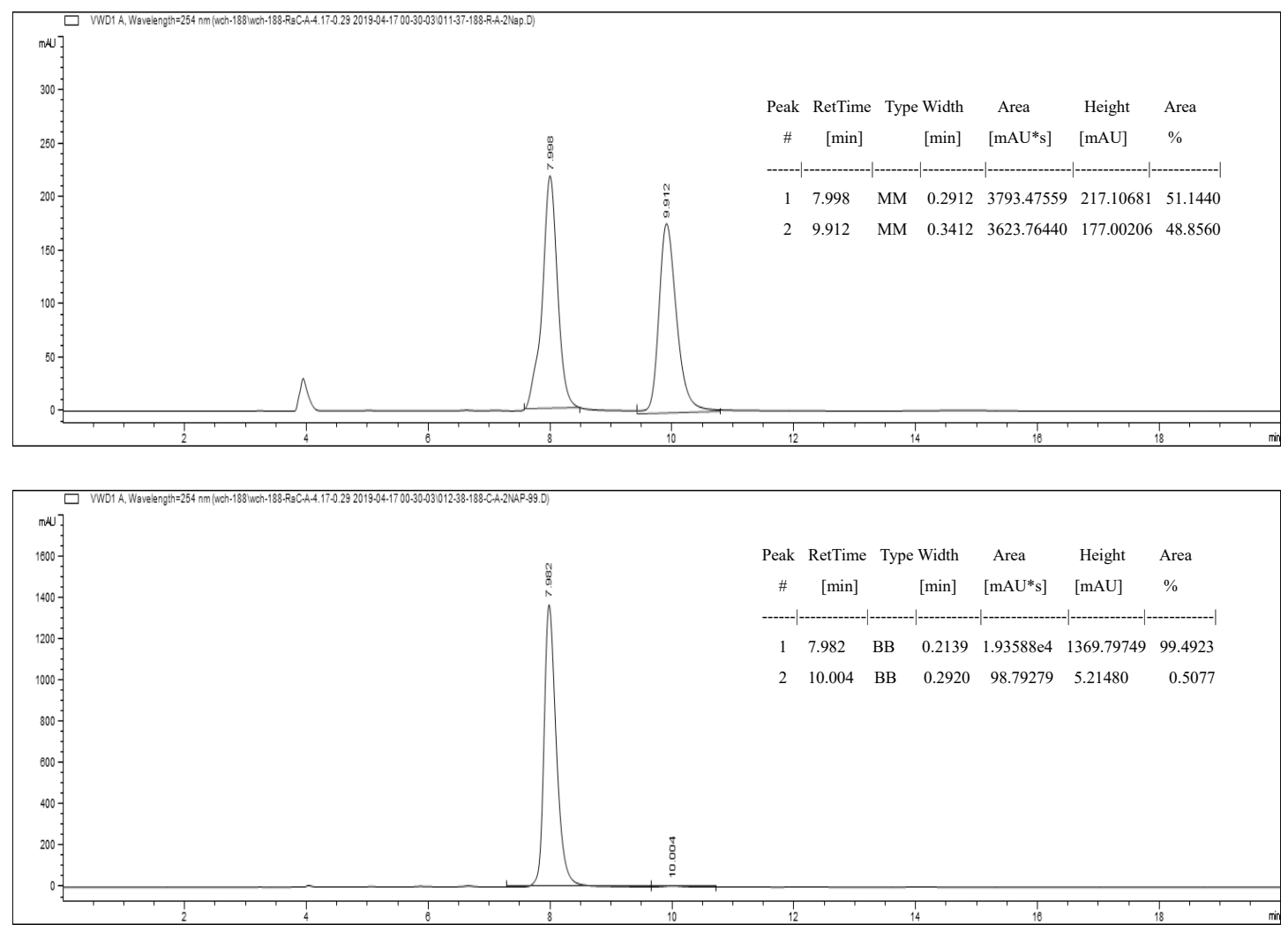
<smiles>CC(=O)N1C=C(C(=O)OCc2cc(C(F)(F)F)cc(C(F)(F)F)c2)[C@H](c2cccs2)[C@H]1c1ccccc1</smiles>

Pale yellow solid, $83 \%$ yield (46 mg). Purified by flash chromatography (12\% EtOAc/PE). $\mathrm{mp}=131-133{ }^{\circ} \mathrm{C} ;[\alpha]{ }_{\mathrm{D}}^{20}=+51.9\left(c 0.44, \mathrm{CH}_{2} \mathrm{Cl}_{2}\right) ;{ }^{1} \mathrm{H}$ NMR $\left(300 \mathrm{MHz}, \mathrm{CDCl}_{3}\right) \delta 8.01(\mathrm{~s}$, 1H), $7.86(\mathrm{~s}, 1 \mathrm{H}), 7.72(\mathrm{~s}, 2 \mathrm{H}), 7.44-7.29(\mathrm{~m}, 3 \mathrm{H}), 7.21-7.08(\mathrm{~m}, 4 \mathrm{H}), 7.04$ (dd, $J=5.1$, $3.6 \mathrm{~Hz}, 1 \mathrm{H}), 5.72(\mathrm{~s}, 1 \mathrm{H}), 5.33(\mathrm{~d}, J=13.1 \mathrm{~Hz}, 1 \mathrm{H}), 5.17(\mathrm{~d}, J=13.1 \mathrm{~Hz}, 1 \mathrm{H}), 3.85(\mathrm{~s}, 3 \mathrm{H})$; ${ }^{13} \mathrm{C}$ NMR $\left(101 \mathrm{MHz}, \mathrm{CDCl}_{3}\right) \delta 162.5,151.8,150.7,144.4,138.3,136.9,132.0(\mathrm{q}, 2 J \mathrm{C}-\mathrm{F}=$ $33.6 \mathrm{~Hz}), 129.4,128.1(\mathrm{q}, 3 J \mathrm{C}-\mathrm{F}=4.1 \mathrm{~Hz}), 127.4,125.7,124.7,124.4,123.1(\mathrm{q}, 1 J \mathrm{C}-\mathrm{F}=$ $273.6 \mathrm{~Hz}), 122.2(\mathrm{sep}, 3 J \mathrm{C}-\mathrm{F}=3.7 \mathrm{~Hz}), 116.9,112.0,72.5,64.7,54.3$; IR (film) $v_{\max } 2959$, $2359,1711,1623,1490,1446,1387,1358,1279,1265,1177,1136,1073,887,764,749,705$ $\mathrm{cm}^{-1}$; HRMS (ESI) calcd for $\mathrm{C}_{25} \mathrm{H}_{19} \mathrm{~F}_{6} \mathrm{~N}_{2} \mathrm{O}_{4} \mathrm{~S}^{+}[\mathrm{M}+\mathrm{H}]^{+}$557.0964, found 557.0953. HPLC analysis: 95\% ee (CHIRALPAK IC, isopropanol/hexane $=10: 90,1.0 \mathrm{~mL} / \mathrm{min}, \mathrm{UV}: 254 \mathrm{~nm}$ ), $t_{\mathrm{R}}=9.392 \min$ (major), $17.888 \min$ (minor).
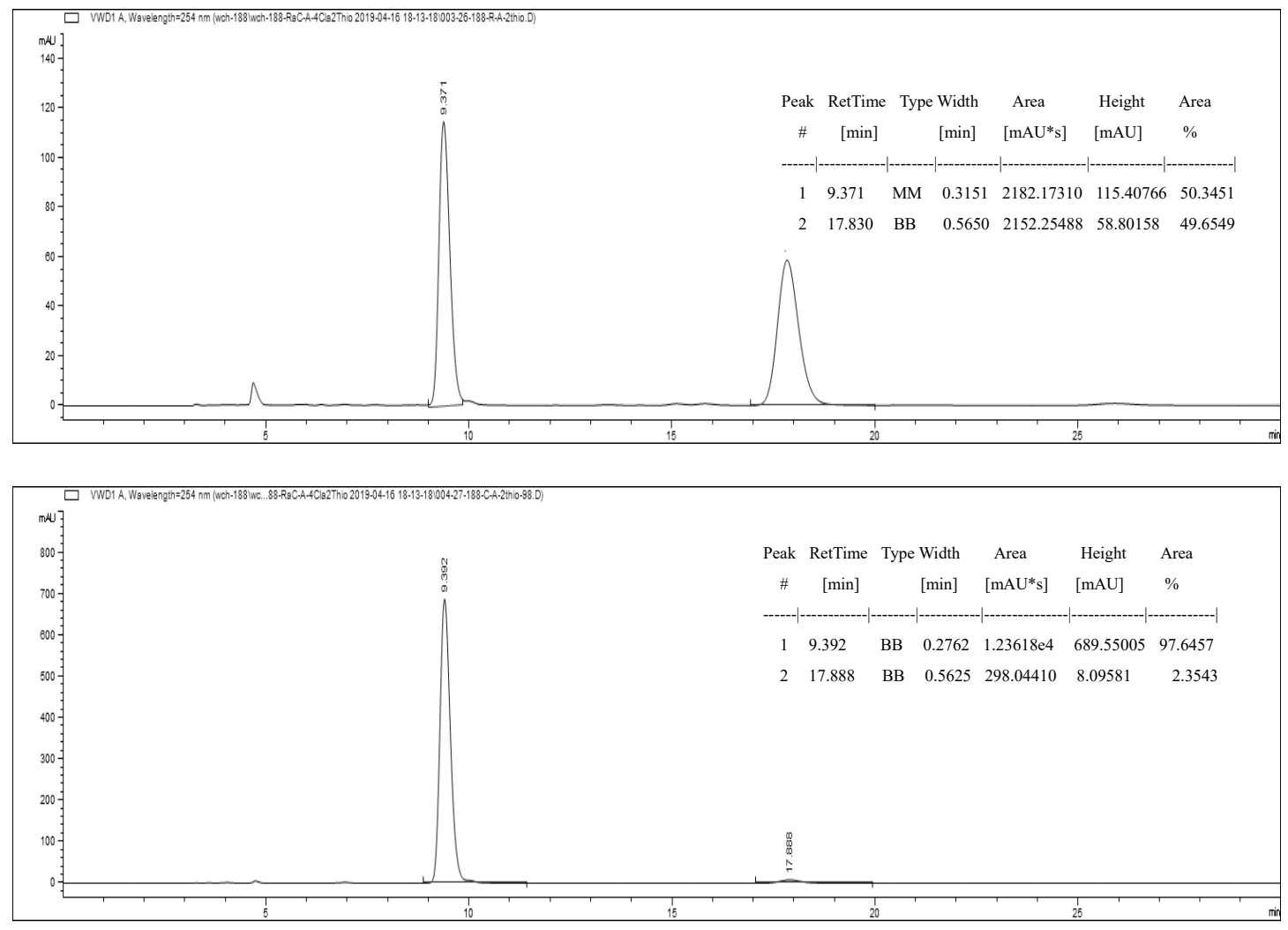
(S)-4-(3,5-bis(trifluoromethyl)benzyl)

\section{pyrazole-1,4-dicarboxylate}<smiles>CC[C@H]1C(C(=O)OCc2cc(C(F)(F)F)cc(C(F)(F)F)c2)=CN(C(C)=O)N1c1ccccc1</smiles>

Viscous liquid, 97\% yield (49 mg). Purified by flash chromatography (12\% EtOAc/PE); $[\alpha]$ ${ }_{\mathrm{D}}^{20}=-92.6\left(c \mathrm{0.46}, \mathrm{CH}_{2} \mathrm{Cl}_{2}\right) ;{ }^{1} \mathrm{H} \mathrm{NMR}\left(300 \mathrm{MHz}, \mathrm{CDCl}_{3}\right) \delta 7.85(\mathrm{~d}, J=15.5 \mathrm{~Hz}, 4 \mathrm{H}), 7.39-$ $7.26(\mathrm{~m}, 2 \mathrm{H}), 7.15-6.96(\mathrm{~m}, 3 \mathrm{H}), 5.32(\mathrm{~d}, J=13.0 \mathrm{~Hz}, 1 \mathrm{H}), 5.23(\mathrm{~d}, J=13.1 \mathrm{~Hz}, 1 \mathrm{H}), 4.44$ (ddd, $J=7.3,3.6,0.8 \mathrm{~Hz}, 1 \mathrm{H}), 3.84$ (s, 3H), $1.93(\mathrm{dtd}, J=14.6,7.3,3.6 \mathrm{~Hz}, 1 \mathrm{H}), 1.74(\mathrm{dt}, J=$ 14.3, 7.2 Hz, 1H), 1.15 (t, $J=7.3 \mathrm{~Hz}, 3 \mathrm{H}) ;{ }^{13} \mathrm{C} \mathrm{NMR}\left(101 \mathrm{MHz}, \mathrm{CDCl}_{3}\right) \delta$ 162.8, 151.4, $138.4,136.8,132.0(\mathrm{q}, 2 J \mathrm{C}-\mathrm{F}=33.5 \mathrm{~Hz}), 129.2,128.2(\mathrm{q}, 3 J \mathrm{C}-\mathrm{F}=4.2 \mathrm{~Hz}), 123.8,123.1(\mathrm{q}$, $1 J \mathrm{C}-\mathrm{F}=272.6 \mathrm{~Hz}), 122.3(\mathrm{sep}, 3 J \mathrm{C}-\mathrm{F}=4.8 \mathrm{~Hz}), 116.7,112.3,75.4,64.5,54.1,27.9,9.4$; IR (film) $v_{\max } 2966,2359,1707,1623,1559,1507,1490,1448,1387,1359,1279,1245,1177$, 1136, 1074, 883, $765 \mathrm{~cm}^{-1}$; HRMS (ESI) calcd for $\mathrm{C}_{23} \mathrm{H}_{20} \mathrm{~F}_{6} \mathrm{~N}_{2} \mathrm{O}_{4}{ }^{+}[\mathrm{M}+\mathrm{H}]^{+} 503.1400$, found 503.1391. HPLC analysis: 77\% ee (CHIRALPAK ID, isopropanol/hexane $=10: 90,1.0$ $\mathrm{mL} / \mathrm{min}, \mathrm{UV}: 254 \mathrm{~nm}$ ), $t_{\mathrm{R}}=5.880 \mathrm{~min}$ (major), $9.813 \mathrm{~min}$ (minor).
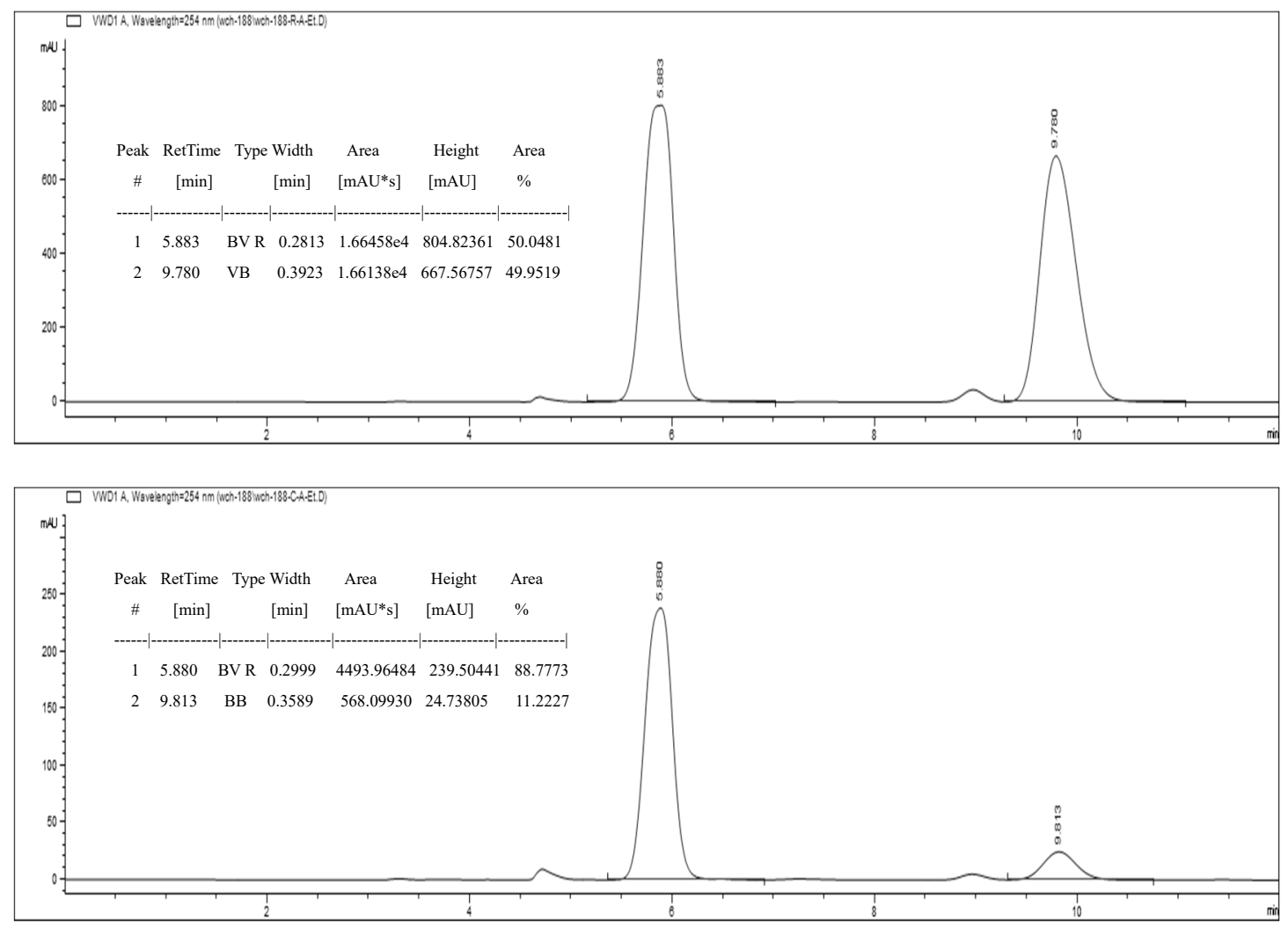
(S)-4-(3,5-bis(trifluoromethyl)benzyl) 1-methyl 3-isopropyl-2-phenyl-2,3-dihydro1H-pyrazole-1,4-dicarboxylate<smiles>CC(=O)N1C=C(C(=O)OCc2cc(C(F)(F)F)cc(C(F)(F)F)c2)[C@@H](C(C)C)N1c1ccccc1</smiles>

Viscous liquid, 91\% yield (47 mg). Purified by flash chromatography (12\% EtOAc/PE); $[\alpha]$ ${ }^{20}{ }_{\mathrm{D}}=-92.6\left(c 0.31, \mathrm{CH}_{2} \mathrm{Cl}_{2}\right) ;{ }^{1} \mathrm{H}$ NMR $\left(300 \mathrm{MHz}, \mathrm{CDCl}_{3}\right) \delta 8.02-7.72(\mathrm{~m}, 4 \mathrm{H}), 7.41-7.24$ (m, 2H), $7.20-6.95(\mathrm{~m}, 3 \mathrm{H}), 5.32(\mathrm{~d}, J=13.1 \mathrm{~Hz}, 1 \mathrm{H}), 5.24$ (d, $J=13.1 \mathrm{~Hz}, 1 \mathrm{H}), 4.36$ (dd, $J$ $=2.8,0.9 \mathrm{~Hz}, 1 \mathrm{H}), 3.84(\mathrm{~s}, 3 \mathrm{H}), 2.10(\mathrm{pd}, J=6.7,2.7 \mathrm{~Hz}, 1 \mathrm{H}), 1.26(\mathrm{~d}, J=6.8 \mathrm{~Hz}, 3 \mathrm{H}), 0.94$ $(\mathrm{d}, J=6.9 \mathrm{~Hz}, 3 \mathrm{H}) ;{ }^{13} \mathrm{C}$ NMR $\left(101 \mathrm{MHz}, \mathrm{CDCl}_{3}\right) \delta 163.0,151.6,138.5,137.2,132.0(\mathrm{q}$, $2 J \mathrm{C}-\mathrm{F}=33.5 \mathrm{~Hz}), 129.2,128.1(\mathrm{q}, 3 J \mathrm{C}-\mathrm{F}=3.7 \mathrm{~Hz}), 123.8,123.1(\mathrm{q}, 1 J \mathrm{C}-\mathrm{F}=273.8 \mathrm{~Hz})$, $122.2(\mathrm{q}, 3 J \mathrm{C}-\mathrm{F}=3.5 \mathrm{~Hz}), 116.9,111.6,64.5,54.1,31.8,19.9,15.6$; IR (film) $v_{\max } \mathrm{cm}^{-1} 2963$, $1705,1621,1491,1445,1385,1357,1276,1244,1171,1128,1073,974,884,843,751$; HRMS (ESI) calcd for $\mathrm{C}_{24} \mathrm{H}_{23} \mathrm{~F}_{6} \mathrm{~N}_{2} \mathrm{O}_{4}{ }^{+}[\mathrm{M}+\mathrm{H}]^{+}$517.1557, found 517.1550. HPLC analysis: $80 \%$ ee $($ CHIRALPAK ID, isopropanol $/$ hexane $=5: 95,1.0 \mathrm{~mL} / \mathrm{min}, \mathrm{UV}: 254 \mathrm{~nm}), t_{\mathrm{R}}=6.083 \mathrm{~min}$ (major), $7.170 \mathrm{~min}$ (minor).
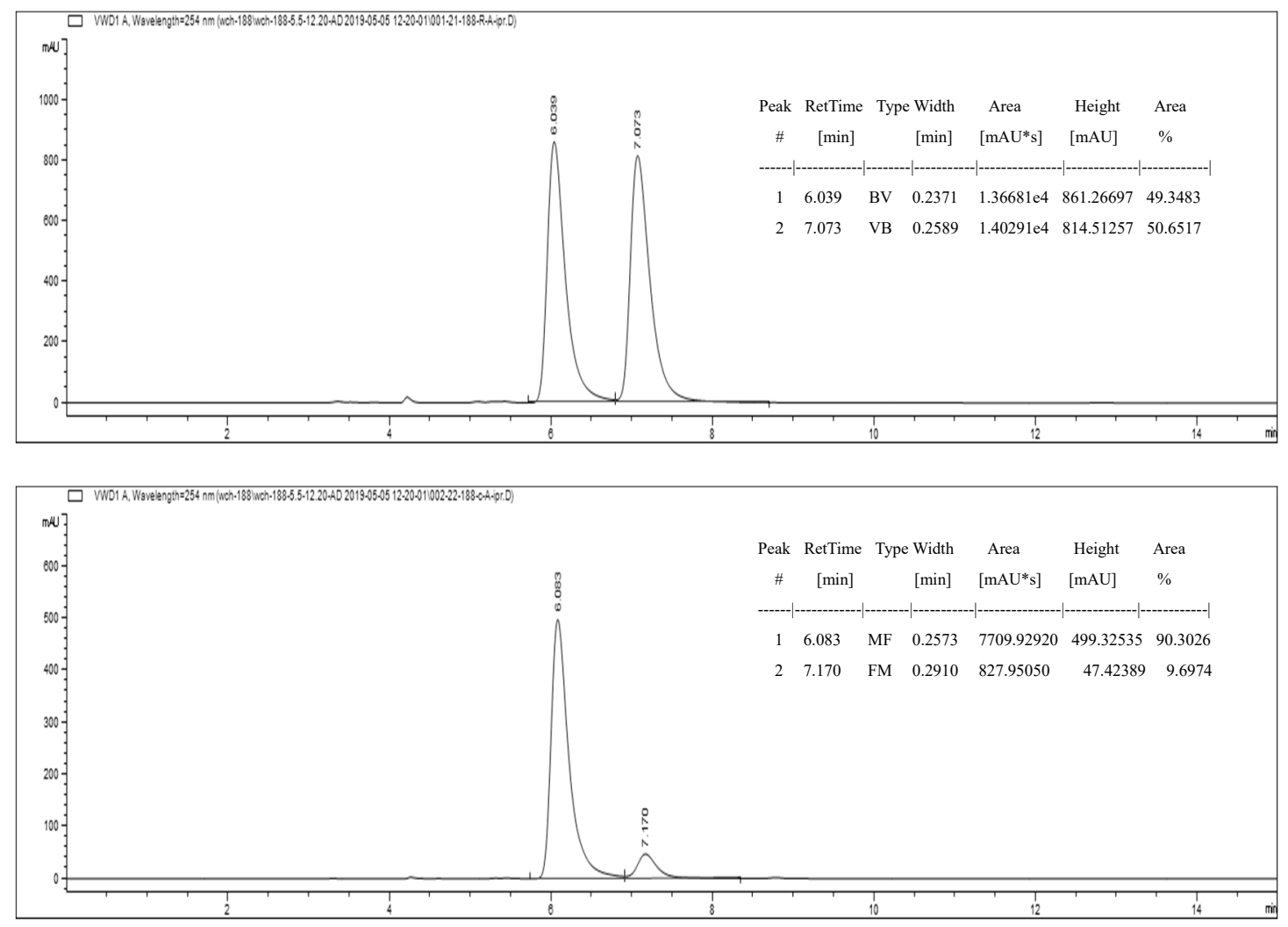
(S)-4-(3,5-bis(trifluoromethyl)benzyl) 1-methyl 2-(2-fluorophenyl)-3-phenyl-2,3-dihydro1H-pyrazole-1,4-dicarboxylate<smiles>CC(=O)N1C=C(C(=O)OCc2cc(C(F)(F)F)cc(C(F)(F)F)c2)[C@H](c2ccccc2)N1c1ccccc1F</smiles>

Pale yellow solid, 97\% yield (55 mg). Purified by flash chromatography (12\% EtOAc/PE). $\mathrm{mp}=114-115{ }^{\circ} \mathrm{C} ;[\alpha]^{20}{ }_{\mathrm{D}}=+131.2\left(c 0.50, \mathrm{CH}_{2} \mathrm{Cl}_{2}\right) ;{ }^{1} \mathrm{H} \mathrm{NMR}\left(300 \mathrm{MHz}, \mathrm{CDCl}_{3}\right) \delta 8.05(\mathrm{~s}$, 1H), $7.84(\mathrm{~s}, 1 \mathrm{H}), 7.66(\mathrm{~s}, 2 \mathrm{H}), 7.50-7.33(\mathrm{~m}, 5 \mathrm{H}), 7.23-7.03$ (m, 4H), 5.37 (d, $J=2.6 \mathrm{~Hz}$, 1H), $5.27(\mathrm{~d}, J=13.1 \mathrm{~Hz}, 1 \mathrm{H}), 5.10(\mathrm{~d}, J=13.1 \mathrm{~Hz}, 1 \mathrm{H}), 3.83(\mathrm{~s}, 3 \mathrm{H}) ;{ }^{13} \mathrm{C}$ NMR $(101 \mathrm{MHz}$, $\left.\mathrm{CDCl}_{3}\right) \delta 162.6,154.6(\mathrm{~d}, 1 J \mathrm{C}-\mathrm{F}=247.6 \mathrm{~Hz}), 139.5,138.5(\mathrm{~d}, 3 J \mathrm{C}-\mathrm{F}=9.1 \mathrm{~Hz}), 138.2,136.5$, $131.9(\mathrm{q}, 2 J \mathrm{C}-\mathrm{F}=33.6 \mathrm{~Hz}), 128.7,128.5,128.0(\mathrm{q}, 3 J \mathrm{C}-\mathrm{F}=3.7 \mathrm{~Hz}), 126.8,125.8(\mathrm{~d}, 3 J \mathrm{C}-\mathrm{F}=$ $7.4 \mathrm{~Hz}), 124.3(\mathrm{~d}, 4 J \mathrm{C}-\mathrm{F}=3.7 \mathrm{~Hz}), 123.3(\mathrm{q}, 1 J \mathrm{C}-\mathrm{F}=259.2 \mathrm{~Hz}), 122.2(\mathrm{sep}, 3 J \mathrm{C}-\mathrm{F}=4.2 \mathrm{~Hz})$, 119.02, 118.98, 117.0 (d, 2JC-F = 19.2 Hz), 113.1, 76.1, 64.6, 54.3; IR (film) $v_{\max } 3058,1711$, $1625,1495,1446,1387,1359,1280,1264,1229,1180,1136,1112,1073,970,890,843,735$, $704 \mathrm{~cm}^{-1}$; HRMS (ESI) calcd for $\mathrm{C}_{27} \mathrm{H}_{20} \mathrm{~F}_{7} \mathrm{~N}_{2} \mathrm{O}_{4}{ }^{+}[\mathrm{M}+\mathrm{H}]^{+}$569.1306, found 569.1296. HPLC analysis: 90\% ee (CHIRALPAK IC, isopropanol/hexane $=10: 90,1.0 \mathrm{~mL} / \mathrm{min}, \mathrm{UV}: 254 \mathrm{~nm}$ ), $t_{\mathrm{R}}=10.066 \min$ (major), $11.007 \mathrm{~min}$ (minor).
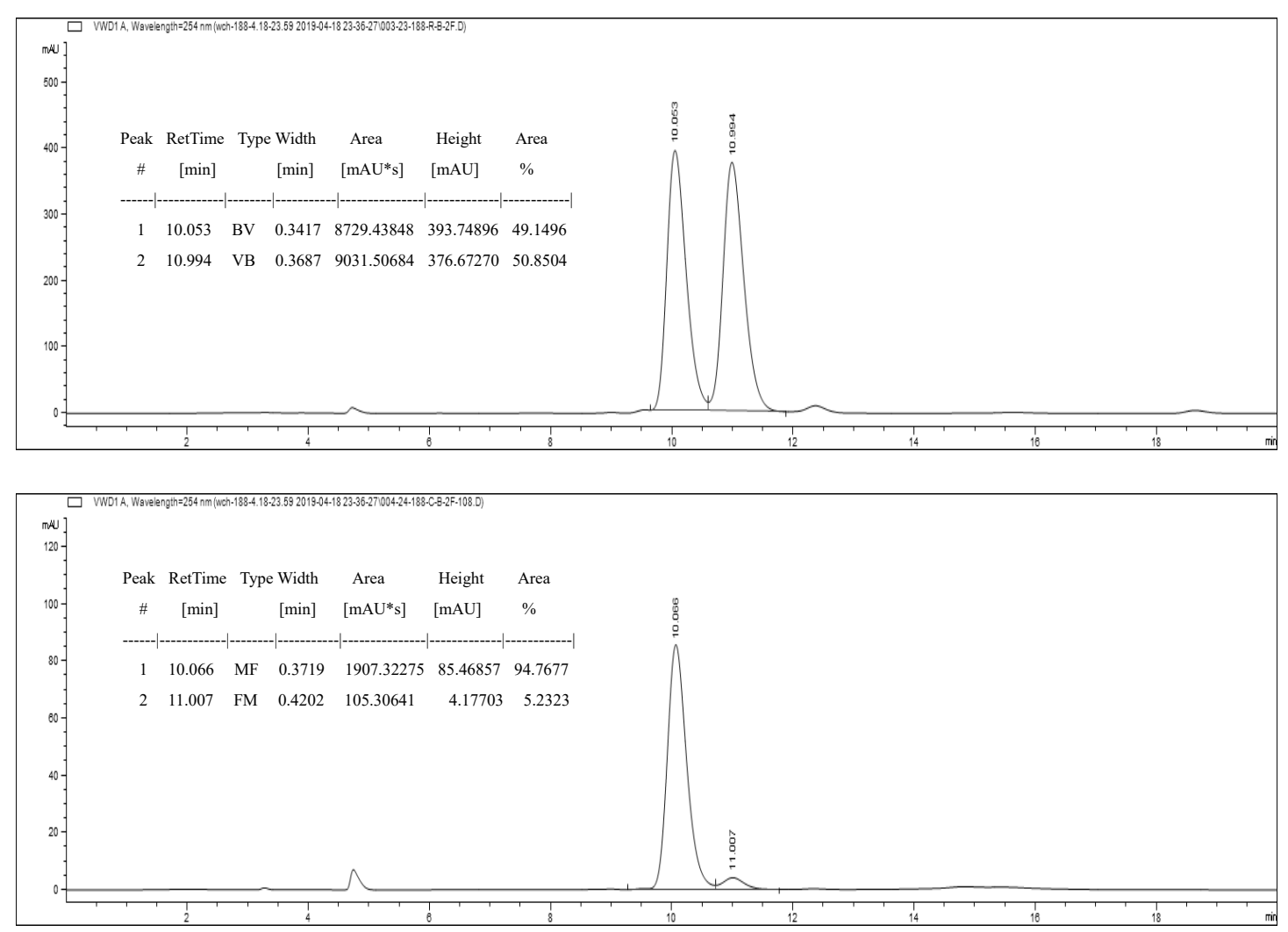
(S)-4-(3,5-bis(trifluoromethyl)benzyl)

2-(3-fluorophenyl)-3-phenyl-2,3-dihydro-1H-pyrazole-1,4-dicarboxylate<smiles>CC(=O)N1C=C(C(=O)OCc2cc(C(F)(F)F)cc(C(F)(F)F)c2)[C@H](c2ccccc2)N1c1cccc(F)c1</smiles>

Pale yellow solid, 99\% yield (56 mg). Purified by flash chromatography (12\% EtOAc/PE). $\mathrm{mp}=105-106{ }^{\circ} \mathrm{C} ;[\alpha]^{20}{ }_{\mathrm{D}}=+92.2\left(c 0.37, \mathrm{CH}_{2} \mathrm{Cl}_{2}\right) ;{ }^{1} \mathrm{H} \mathrm{NMR}\left(300 \mathrm{MHz}, \mathrm{CDCl}_{3}\right) \delta 7.98(\mathrm{~d}$, $J=1.1 \mathrm{~Hz}, 1 \mathrm{H}), 7.85(\mathrm{~s}, 1 \mathrm{H}), 7.67(\mathrm{~s}, 2 \mathrm{H}), 7.48-7.27$ (m, 6H), $6.88-6.67$ (m, 3H), $5.46(\mathrm{~d}$, $J=1.1 \mathrm{~Hz}, 1 \mathrm{H}), 5.28(\mathrm{~d}, J=13.0 \mathrm{~Hz}, 1 \mathrm{H}), 5.11(\mathrm{~d}, J=13.1 \mathrm{~Hz}, 1 \mathrm{H}), 3.90(\mathrm{~s}, 3 \mathrm{H}) ;{ }^{13} \mathrm{C} \mathrm{NMR}$ $\left(101 \mathrm{MHz}, \mathrm{CDCl}_{3}\right) \delta 163.4(\mathrm{~d}, 1 J \mathrm{C}-\mathrm{F}=246.2 \mathrm{~Hz}), 162.4,153.2(\mathrm{~d}, 3 J \mathrm{C}-\mathrm{F}=8.8 \mathrm{~Hz}), 152.0$, 140.0, 138.1, 136.3, 132.0 (q, 2JC-F = 33.5 Hz), $130.6(\mathrm{~d}, 3 J \mathrm{C}-\mathrm{F}=9.4 \mathrm{~Hz}), 129.0,128.6$, $128.1(\mathrm{q}, 3 J \mathrm{C}-\mathrm{F}=3.9 \mathrm{~Hz}), 126.6,123.1(\mathrm{q}, 2 J \mathrm{C}-\mathrm{F}=273.8 \mathrm{~Hz}), 122.3(\mathrm{sep}, 3 J \mathrm{C}-\mathrm{F}=3.5 \mathrm{~Hz})$, 113.2, $112.2(\mathrm{~d}, 4 J \mathrm{C}-\mathrm{F}=2.9 \mathrm{~Hz}), 110.7(\mathrm{~d}, 2 J \mathrm{C}-\mathrm{F}=21.5 \mathrm{~Hz}), 104.1(\mathrm{~d}, 2 J \mathrm{C}-\mathrm{F}=25.5 \mathrm{~Hz})$, 76.8, 64.7, 54.4; IR (film) $v_{\max } 2960,1713,1611,1593,1488,1446,1386,1356,1280,1235$, $1176,1137,1074,1005,989,884,844,789,766 \mathrm{~cm}^{-1}$; HRMS (ESI) calcd for $\mathrm{C}_{27} \mathrm{H}_{20} \mathrm{~F}_{7} \mathrm{~N}_{2} \mathrm{O}_{4}{ }^{+}$ $[\mathrm{M}+\mathrm{H}]^{+}$569.1306, found 569.1294. HPLC analysis: 97\% ee (CHIRALPAK IC, isopropanol/hexane $=10: 90,1.0 \mathrm{~mL} / \mathrm{min}, \mathrm{UV}: 254 \mathrm{~nm}$ ), $t_{\mathrm{R}}=6.358 \mathrm{~min}$ (major), $11.320 \mathrm{~min}$ (minor).
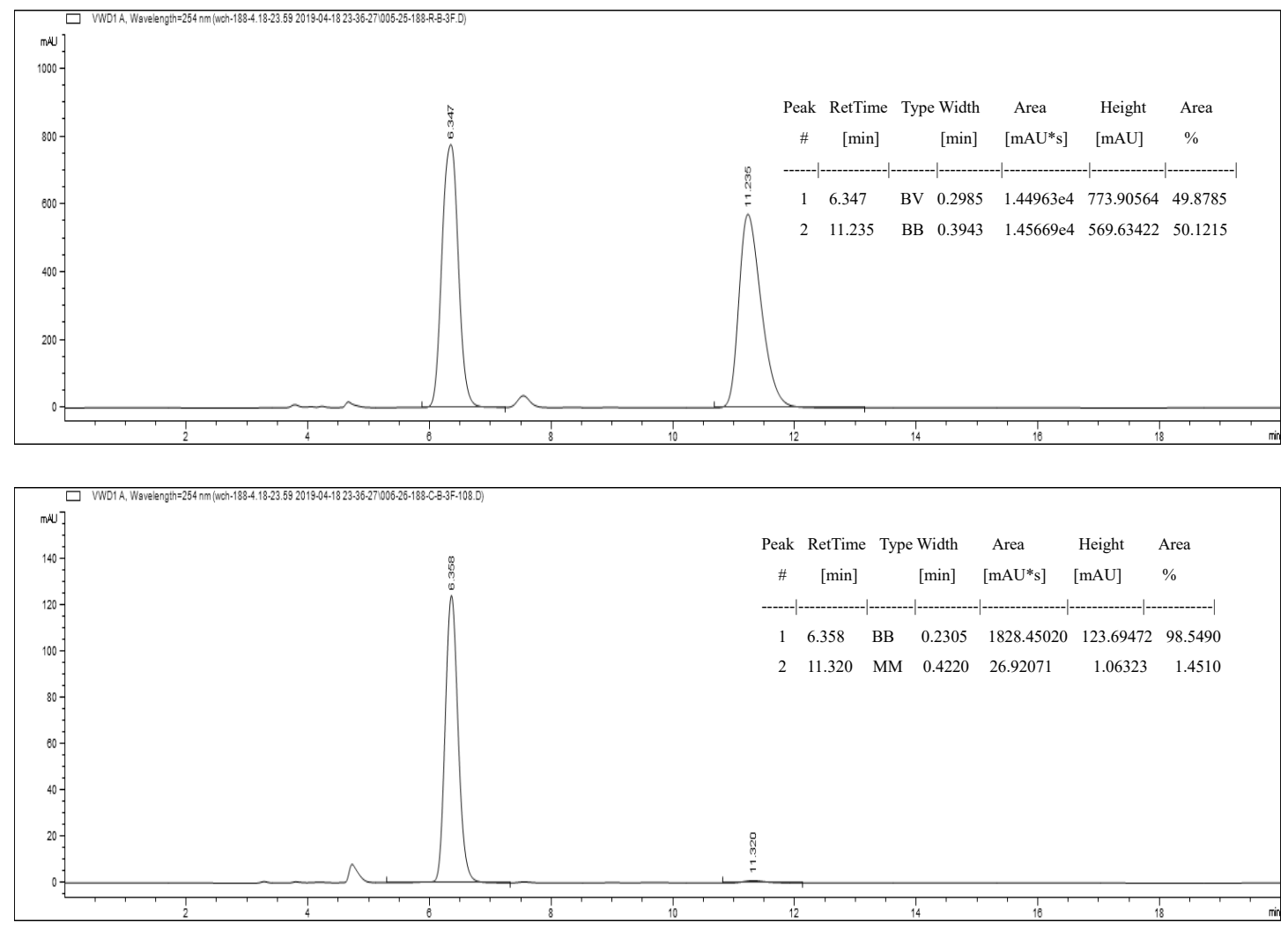
(S)-4-(3,5-bis(trifluoromethyl)benzyl)<smiles>CC(=O)N1C=C(C(=O)OCc2cc(C(F)(F)F)cc(C(F)(F)F)c2)C(c2ccccc2)N1c1ccc(F)cc1</smiles>

White solid, $96 \%$ yield $(54 \mathrm{mg})$. Purified by flash chromatography $(12 \%$ EtOAc/PE). $\mathrm{mp}=$ $151-152{ }^{\circ} \mathrm{C} ;[\alpha]{ }_{\mathrm{D}}^{20}=+95.5\left(c 0.40, \mathrm{CH}_{2} \mathrm{Cl}_{2}\right) ;{ }^{1} \mathrm{H}$ NMR $\left(300 \mathrm{MHz}, \mathrm{CDCl}_{3}\right) \delta 8.00(\mathrm{~d}, J=$ $1.0 \mathrm{~Hz}, 1 \mathrm{H}), 7.84(\mathrm{~s}, 1 \mathrm{H}), 7.66(\mathrm{~s}, 2 \mathrm{H}), 7.47-7.32(\mathrm{~m}, 5 \mathrm{H}), 7.12-6.97(\mathrm{~m}, 4 \mathrm{H}), 5.34(\mathrm{~d}, J=$ $1.1 \mathrm{~Hz}, 1 \mathrm{H}), 5.27(\mathrm{~d}, J=13.1 \mathrm{~Hz}, 1 \mathrm{H}), 5.10(\mathrm{~d}, J=13.1 \mathrm{~Hz}, 1 \mathrm{H}), 3.86(\mathrm{~s}, 3 \mathrm{H}) ;{ }^{13} \mathrm{C}$ NMR $(126$ $\left.\mathrm{MHz}, \mathrm{CDCl}_{3}\right) \delta 161.50,158.66(\mathrm{~d}, 2 J \mathrm{C}-\mathrm{F}=243.1 \mathrm{~Hz}), 150.73,146.41(\mathrm{~d}, 4 J \mathrm{C}-\mathrm{F}=2.6 \mathrm{~Hz})$, 139.30, 137.11, 135.32, $130.90(\mathrm{q}, 2 J \mathrm{C}-\mathrm{F}=33.5 \mathrm{~Hz}), 127.92,127.55,127.03(\mathrm{q}, 3 J \mathrm{C}-\mathrm{F}=3.8$ Hz), 125.58, $121.20(\mathrm{sep}, 3 J \mathrm{C}-\mathrm{F}=3.6 \mathrm{~Hz}), 117.85(\mathrm{~d}, 3 J \mathrm{C}-\mathrm{F}=8.2 \mathrm{~Hz}), 115.03(\mathrm{~d}, 2 J \mathrm{C}-\mathrm{F}=$ $22.7 \mathrm{~Hz}), 111.28,76.22,63.59,53.27 .122 .04(\mathrm{q}, 1 J \mathrm{C}-\mathrm{F}=272.8 \mathrm{~Hz})$; IR (film) $v_{\max } 2961$, 1708, 1623, 1504, 1444, 1386, 1356, 1277, 1247, 1171, 1127, 1072, 1011, 969, 885, 843, 828, 807, $750 \mathrm{~cm}^{-1}$; HRMS (ESI) calcd for $\mathrm{C}_{27} \mathrm{H}_{20} \mathrm{~F}_{7} \mathrm{~N}_{2} \mathrm{O}_{4}{ }^{+}[\mathrm{M}+\mathrm{H}]^{+} 569.1306$, found 569.1296. HPLC analysis: $91 \%$ ee (CHIRALPAK IC, isopropanol/hexane $=10: 90,1.0 \mathrm{~mL} / \mathrm{min}, \mathrm{UV}$ : $254 \mathrm{~nm}$ ), $t_{\mathrm{R}}=7.158 \min$ (major), $9.598 \min$ (minor).
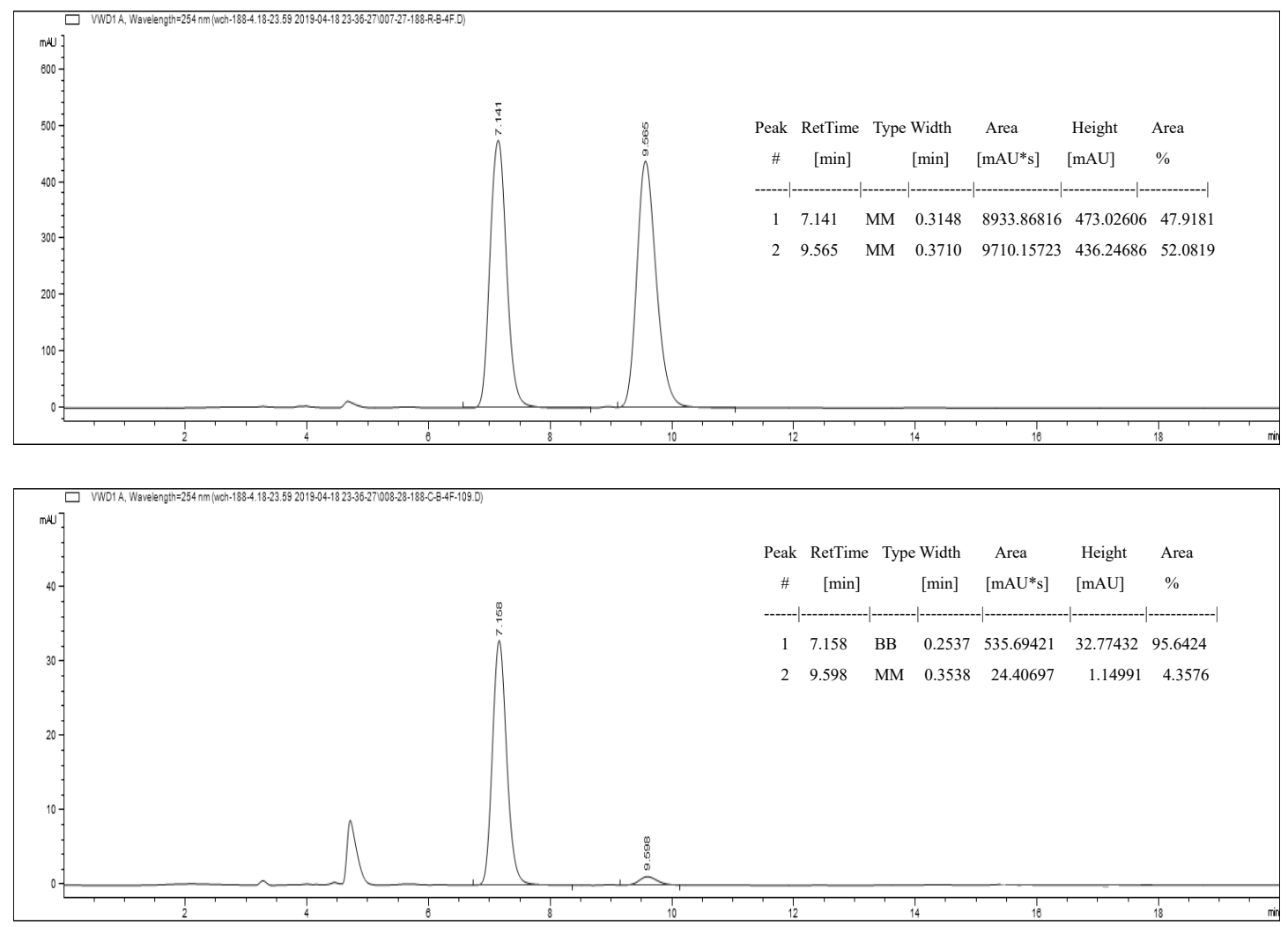
(S)-4-(3,5-bis(trifluoromethyl)benzyl) 1-methyl 2-(2,4-difluorophenyl)-3-phenyl-2,3-dihydro-1H-pyrazole-1,4-dicarboxylate<smiles>CC(=O)N1C=C(C(=O)OCc2cc(C(F)(F)F)cc(C(F)(F)F)c2)[C@H](c2ccccc2)[C@H]1c1ccc(F)c(F)c1</smiles>

Yellow solid, 95\% yield (56 mg). Purified by flash chromatography (12\% EtOAc/PE). mp $=$ $99-101{ }^{\circ} \mathrm{C} ;[\alpha]^{20}{ }_{\mathrm{D}}=+85.0\left(c 0.27, \mathrm{CH}_{2} \mathrm{Cl}_{2}\right) ;{ }^{1} \mathrm{H}$ NMR $\left(300 \mathrm{MHz}, \mathrm{CDCl}_{3}\right) \delta 7.96(\mathrm{~d}, J=1.1$ $\mathrm{Hz}, 1 \mathrm{H}), 7.84(\mathrm{~s}, 1 \mathrm{H}), 7.66(\mathrm{~s}, 2 \mathrm{H}), 7.46-7.33(\mathrm{~m}, 5 \mathrm{H}), 7.14$ (dt, $J=9.7,8.7 \mathrm{~Hz}, 1 \mathrm{H}), 6.94-$ $6.72(\mathrm{~m}, 2 \mathrm{H}), 5.36(\mathrm{~d}, J=1.2 \mathrm{~Hz}, 1 \mathrm{H}), 5.27(\mathrm{~d}, J=13.1 \mathrm{~Hz}, 1 \mathrm{H}), 5.10(\mathrm{~d}, J=13.1 \mathrm{~Hz}, 1 \mathrm{H})$, $3.88(\mathrm{~s}, 3 \mathrm{H}) ;{ }^{13} \mathrm{C} \mathrm{NMR}\left(126 \mathrm{MHz}, \mathrm{CDCl}_{3}\right) \delta 161.3,150.8,149.5(\mathrm{dd}, 1 J \mathrm{C}-\mathrm{F}=248.9 \mathrm{~Hz}$, $2 J \mathrm{C}-\mathrm{F}=13.6 \mathrm{~Hz}), 146.9(\mathrm{dd}, 3 J \mathrm{C}-\mathrm{F}=6.4 \mathrm{~Hz}, 4 J \mathrm{C}-\mathrm{F}=2.5 \mathrm{~Hz}), 146.1(\mathrm{dd}, 1 J \mathrm{C}-\mathrm{F}=245.1 \mathrm{~Hz}$, $2 J \mathrm{C}-\mathrm{F}=12.8 \mathrm{~Hz}), 138.9,137.0,135.2,130.9(\mathrm{q}, 2 J \mathrm{C}-\mathrm{F}=33.5 \mathrm{~Hz}), 128.0,127.7,127.1(\mathrm{q}$, $3 J \mathrm{C}-\mathrm{F}=4.1 \mathrm{~Hz}), 125.5,121.3(\mathrm{sep}, 3 J \mathrm{C}-\mathrm{F}=4.1 \mathrm{~Hz}), 122.0(\mathrm{q}, 1 J \mathrm{C}-\mathrm{F}=272.8 \mathrm{~Hz}), 116.7(\mathrm{~d}$, $2 J \mathrm{C}-\mathrm{F}=18.3 \mathrm{~Hz}), 111.8(\mathrm{dd}, 3 J \mathrm{C}-\mathrm{F}=6.1 \mathrm{~Hz}, 4 J \mathrm{C}-\mathrm{F}=3.4 \mathrm{~Hz}), 111.7,105.7(\mathrm{~d}, 2 J \mathrm{C}-\mathrm{F}=20.5$ Hz), 76.1, 63.7, 53.4; IR (film) $v_{\max } 2963,2360,1715,1623,1515,1445,1387,1356,1279$, 1260, 1238, 1176, 1135, 1074, 987, 887, 843, $750 \mathrm{~cm}^{-1}$; HRMS (ESI) calcd for $\mathrm{C}_{27} \mathrm{H}_{19} \mathrm{~F}_{8} \mathrm{~N}_{2} \mathrm{O}_{4}{ }^{+}[\mathrm{M}+\mathrm{H}]^{+}$587.1212, found 587.1202. HPLC analysis: 92\% ee (CHIRALPAK IC, isopropanol/hexane $=10: 90,1.0 \mathrm{~mL} / \mathrm{min}, \mathrm{UV}: 254 \mathrm{~nm}$ ), $\mathrm{tR}=6.684 \mathrm{~min}$ (major), $9.270 \mathrm{~min}$ (minor).
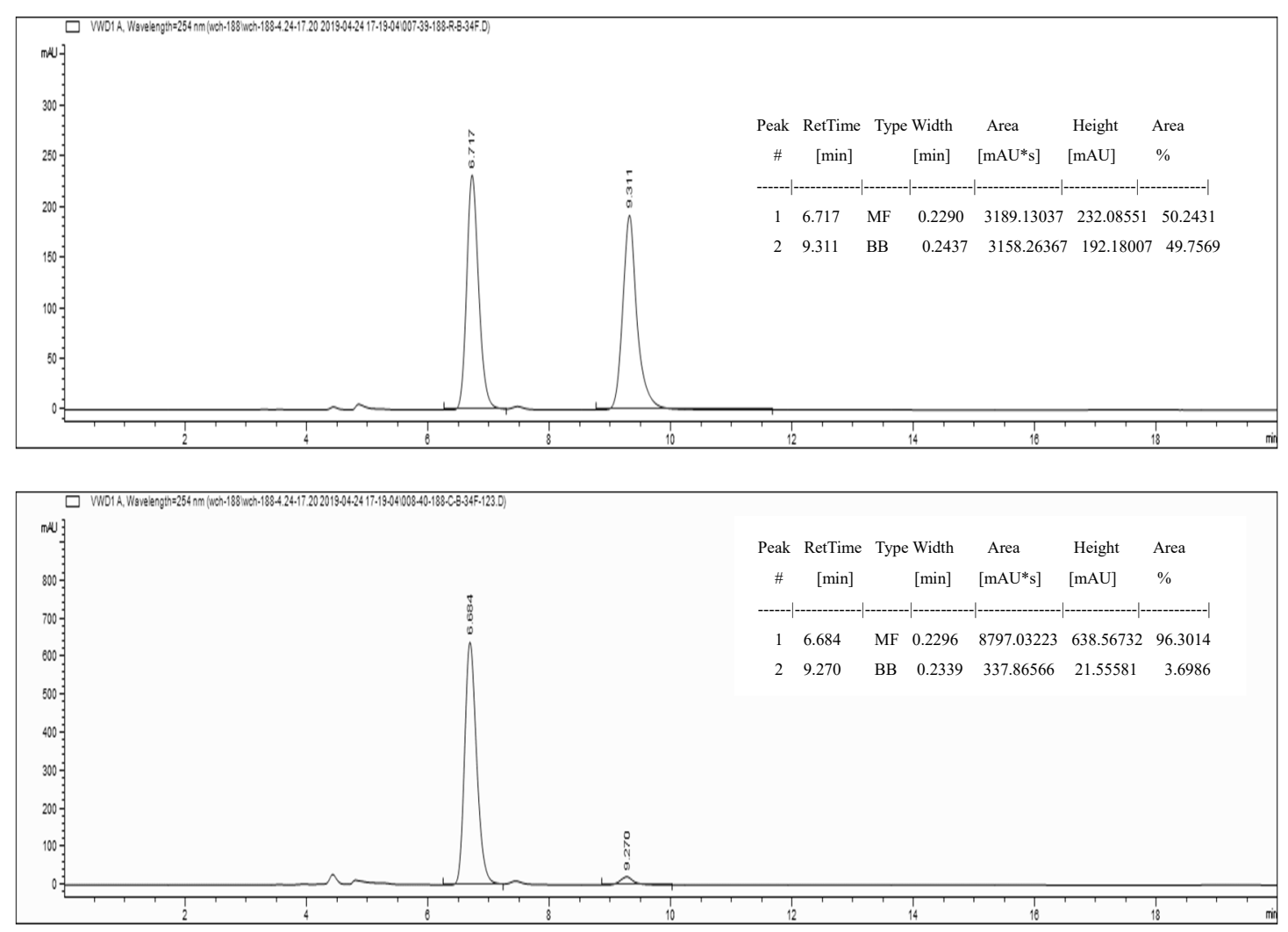
(S)-4-(3,5-bis(trifluoromethyl)benzyl)

1-methyl 2-(3-chlorophenyl)-3-phenyl-2,3-dihydro-1H-pyrazole-1,4-dicarboxylate<smiles>CC(=O)N1C=C(C(=O)OCc2cc(C(F)(F)F)cc(C(F)(F)F)c2)[C@H](c2ccccc2)N1c1cccc(Cl)c1</smiles>

Pale yellow solid, $87 \%$ yield $(51 \mathrm{mg})$. Purified by flash chromatography (12\% EtOAc/PE). $\mathrm{mp}=53-54{ }^{\circ} \mathrm{C} ;[\alpha]^{20}{ }_{\mathrm{D}}=+87.5\left(c 0.32, \mathrm{CH}_{2} \mathrm{Cl}_{2}\right) ;{ }^{1} \mathrm{H} \mathrm{NMR}\left(300 \mathrm{MHz}, \mathrm{CDCl}_{3}\right) \delta 7.97(\mathrm{~d}, J=$ $1.1 \mathrm{~Hz}, 1 \mathrm{H}), 7.84(\mathrm{~s}, 1 \mathrm{H}), 7.67(\mathrm{~d}, J=1.5 \mathrm{~Hz}, 2 \mathrm{H}), 7.47-7.34(\mathrm{~m}, 5 \mathrm{H}), 7.26(\mathrm{~d}, J=8.1 \mathrm{~Hz}$, 1H), 7.09 (ddd, $J=7.9,2.0,0.9 \mathrm{~Hz}, 1 \mathrm{H}), 7.01$ (t, $J=2.1 \mathrm{~Hz}, 1 \mathrm{H}), 6.92$ (ddd, $J=8.2,2.4,1.0$ Hz, 1H), $5.43(\mathrm{~d}, J=1.1 \mathrm{~Hz}, 1 \mathrm{H}), 5.27$ (d, $J=13.0 \mathrm{~Hz}, 1 \mathrm{H}), 5.10(\mathrm{~d}, J=13.0 \mathrm{~Hz}, 1 \mathrm{H}), 3.89(\mathrm{~s}$, $3 \mathrm{H}) ;{ }^{13} \mathrm{C} \mathrm{NMR}\left(101 \mathrm{MHz}, \mathrm{CDCl}_{3}\right) \delta 162.4,152.5,151.9,140.0,138.1,136.3,135.1,132.0$ (q, $2 J \mathrm{C}-\mathrm{F}=33.5 \mathrm{~Hz}), 130.4,129.0,128.7,128.2(\mathrm{q}, 3 J \mathrm{C}-\mathrm{F}=4.0 \mathrm{~Hz}), 126.5,124.1,123.1(\mathrm{q}$, $1 J \mathrm{C}-\mathrm{F}=272.7 \mathrm{~Hz}), 122.3(\mathrm{sep}, 3 \mathrm{JC}-\mathrm{F}=3.6 \mathrm{~Hz}), 116.8,115.0,113.1,76.8,64.7,54.5$; IR (film) $v_{\max } 2961,1713,1625,1593,1476,1444,1386,1356,1279,1248,1178,1134,1074$, 978, 886, 843, $749 \mathrm{~cm}^{-1}$; HRMS (ESI) calcd for $\mathrm{C}_{27} \mathrm{H}_{20} \mathrm{ClF}_{6} \mathrm{~N}_{2} \mathrm{O}_{4}{ }^{+}[\mathrm{M}+\mathrm{H}]^{+}$585.101, found 585.0999. HPLC analysis: 98\% ee (CHIRALPAK IC, isopropanol/hexane $=10: 90,1.0$ $\mathrm{mL} / \mathrm{min}, \mathrm{UV}: 254 \mathrm{~nm}$ ), $t_{\mathrm{R}}=6.299 \min$ (major), $14.275 \mathrm{~min}$ (minor).
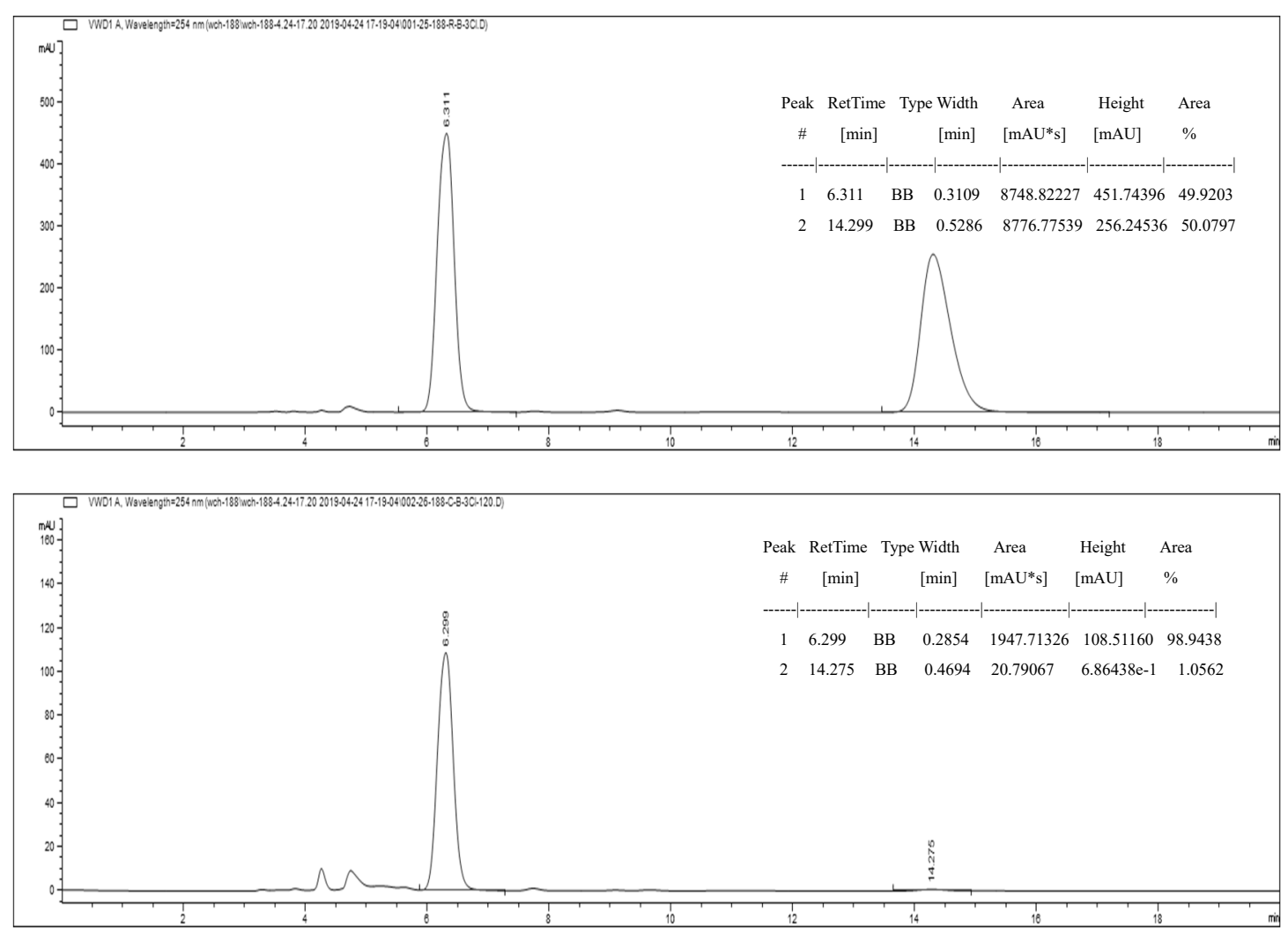
<smiles>CC(=O)N1C=C(C(=O)OCc2cc(C(F)(F)F)cc(C(F)(F)F)c2)[C@H](c2ccccc2)N1c1ccc(Cl)cc1</smiles>

Pale yellow solid, 94\% yield (55 mg). Purified by flash chromatography (12\% EtOAc/PE). $\mathrm{mp}=85-86{ }^{\circ} \mathrm{C} ;[\alpha]^{20}{ }_{\mathrm{D}}=+138.8\left(c 0.50, \mathrm{CH}_{2} \mathrm{Cl}_{2}\right) ;{ }^{1} \mathrm{H}$ NMR $\left(300 \mathrm{MHz}, \mathrm{CDCl}_{3}\right) \delta 7.99(\mathrm{~d}, J$ $=1.1 \mathrm{~Hz}, 1 \mathrm{H}), 7.85(\mathrm{~s}, 1 \mathrm{H}), 7.67(\mathrm{~d}, J=1.7 \mathrm{~Hz}, 2 \mathrm{H}), 7.48-7.36(\mathrm{~m}, 5 \mathrm{H}), 7.34-7.29(\mathrm{~m}, 2 \mathrm{H})$, $7.04-6.90$ (m, 2H), 5.39 (d, $J=1.1 \mathrm{~Hz}, 1 \mathrm{H}), 5.27$ (d, $J=13.1 \mathrm{~Hz}, 1 \mathrm{H}), 5.10(\mathrm{~d}, J=13.1 \mathrm{~Hz}$, 1H), $3.88(\mathrm{~s}, 3 \mathrm{H}) ;{ }^{13} \mathrm{C} \mathrm{NMR}\left(101 \mathrm{MHz}, \mathrm{CDCl}_{3}\right) \delta 162.4,151.8,149.9,140.2,138.1,136.3$, $132.0(\mathrm{q}, 2 J \mathrm{C}-\mathrm{F}=33.5 \mathrm{~Hz}), 129.34,129.28,129.0,128.6,128.1(\mathrm{q}, 3 J \mathrm{C}-\mathrm{F}=3.6 \mathrm{~Hz}), 126.6$, $123.1(\mathrm{q}, 1 J \mathrm{C}-\mathrm{F}=272.8 \mathrm{~Hz}), 122.3(\mathrm{sep}, 3 J \mathrm{C}-\mathrm{F}=3.5 \mathrm{~Hz}), 118.2,112.8,77.2,64.7,54.4$; IR (film) $v_{\max } 2959,1712,1625,1489,1445,1386,1357,1280,1249,1178,1136,1075,1008$, 970, 885, 844, 823, $765 \mathrm{~cm}^{-1}$; HRMS (ESI) calcd for $\mathrm{C}_{27} \mathrm{H}_{20} \mathrm{ClF}_{6} \mathrm{~N}_{2} \mathrm{O}_{4}{ }^{+}[\mathrm{M}+\mathrm{H}]^{+}$585.1010, found 585.0999. HPLC analysis: 95\% ee (CHIRALPAK IC, isopropanol/hexane $=10: 90,1.0$ $\mathrm{mL} / \mathrm{min}, \mathrm{UV}: 254 \mathrm{~nm}$ ), $t_{\mathrm{R}}=6.929 \min$ (major), $9.088 \mathrm{~min}$ (minor).
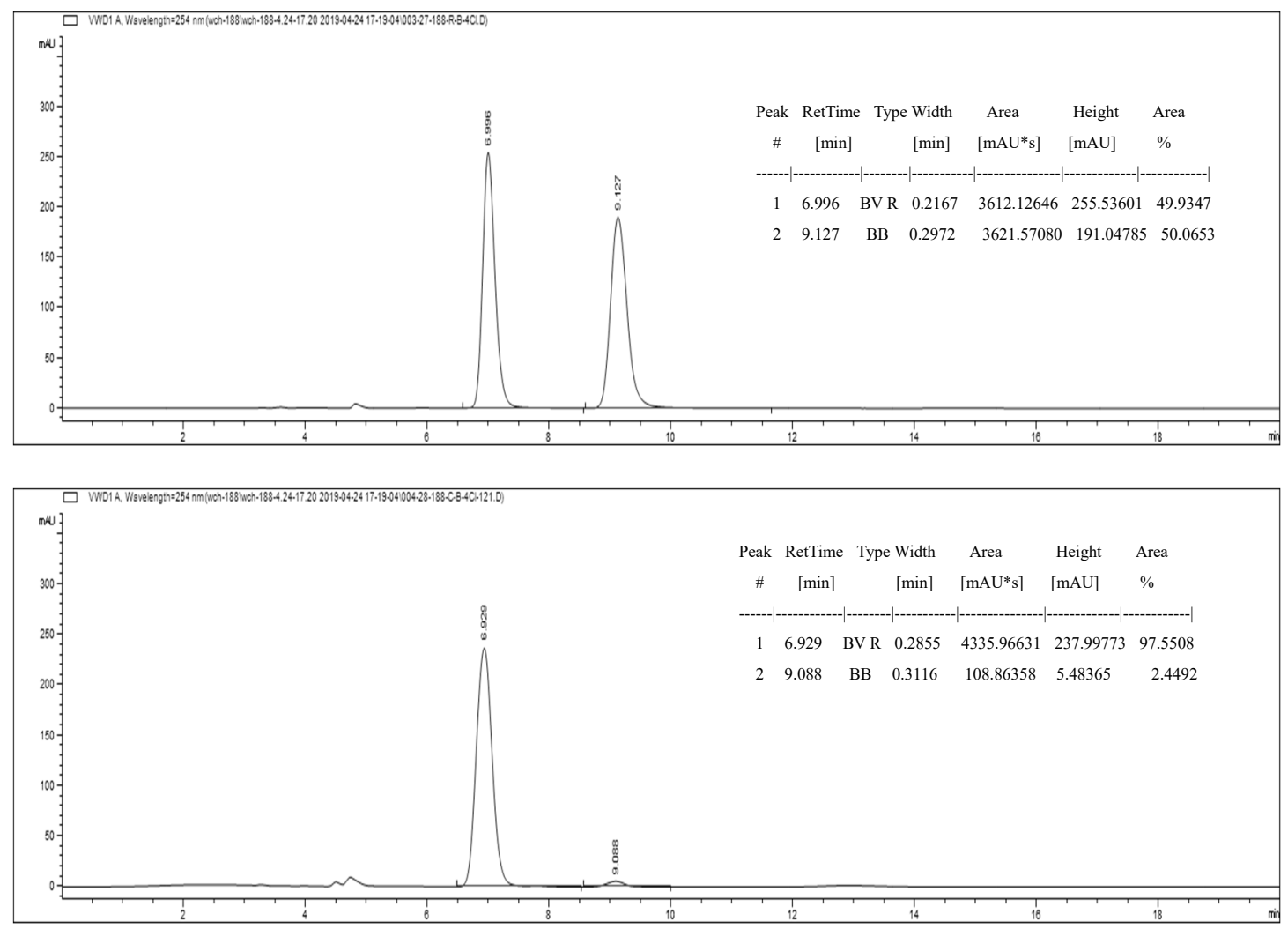
(S)-4-(3,5-bis(trifluoromethyl)benzyl)

1-methyl 2-(2-bromophenyl)-3-phenyl-2,3-dihydro-1H-pyrazole-1,4-dicarboxylate<smiles>CC(=O)N1C=C(C(=O)OCc2cc(C(F)(F)F)cc(C(F)(F)F)c2)[C@H](c2ccccc2)N1c1ccccc1Br</smiles>

Pale yellow solid, 91\% yield $(57 \mathrm{mg})$. Purified by flash chromatography (12\% EtOAc/PE). $\mathrm{mp}=46-47{ }^{\circ} \mathrm{C} ;[\alpha]^{20}{ }_{\mathrm{D}}=+68.9\left(c 0.55, \mathrm{CH}_{2} \mathrm{Cl}_{2}\right) ;{ }^{1} \mathrm{H}$ NMR $\left(300 \mathrm{MHz}, \mathrm{CDCl}_{3}\right) \delta 7.97(\mathrm{~d}, J=$ $1.1 \mathrm{~Hz}, 1 \mathrm{H}), 7.85(\mathrm{~s}, 1 \mathrm{H}), 7.67(\mathrm{~d}, J=1.7 \mathrm{~Hz}, 2 \mathrm{H}), 7.49-7.34(\mathrm{~m}, 5 \mathrm{H}), 7.28-7.14(\mathrm{~m}, 3 \mathrm{H})$, $7.01-6.91(\mathrm{~m}, 1 \mathrm{H}), 5.43(\mathrm{~d}, J=1.1 \mathrm{~Hz}, 1 \mathrm{H}), 5.28(\mathrm{~d}, J=13.0 \mathrm{~Hz}, 1 \mathrm{H}), 5.11(\mathrm{~d}, J=13.0 \mathrm{~Hz}$, 1H), $3.89(\mathrm{~s}, 3 \mathrm{H}) ;{ }^{13} \mathrm{C} \mathrm{NMR}\left(101 \mathrm{MHz}, \mathrm{CDCl}_{3}\right) \delta 162.4,152.6,151.9,140.0,138.1,136.3$, $132.0(\mathrm{q}, 2 J \mathrm{C}-\mathrm{F}=33.5 \mathrm{~Hz}), 130.7,129.0,128.6,128.1(\mathrm{q}, 3 J \mathrm{C}-\mathrm{F}=4.0 \mathrm{~Hz}), 127.1,126.5$, $123.09,123.06(\mathrm{q}, 1 J \mathrm{C}-\mathrm{F}=273.8 \mathrm{~Hz}), 122.3(\mathrm{sep}, 3 J \mathrm{C}-\mathrm{F}=4.0 \mathrm{~Hz}), 119.7,115.6,113.1,76.8$, 64.7, 54.4; IR (film) $v_{\max } 2959,1713,1626,1590,1474,1445,1386,1356,1280,1248,1180$, 1136, 1074, 979, 883, 844, $765 \mathrm{~cm}^{-1}$; HRMS (ESI) calcd for $\mathrm{C}_{27} \mathrm{H}_{20} \mathrm{BrF}_{6} \mathrm{~N}_{2} \mathrm{O}_{4}{ }^{+}[\mathrm{M}+\mathrm{H}]^{+}$ 629.0505, found 629.0496. HPLC analysis: 98\% ee (CHIRALPAK ID, isopropanol/hexane = 10:90, $1.0 \mathrm{~mL} / \mathrm{min}, \mathrm{UV}: 254 \mathrm{~nm}$ ), $t_{\mathrm{R}}=6.729 \min$ (major), $7.568 \mathrm{~min}$ (minor).
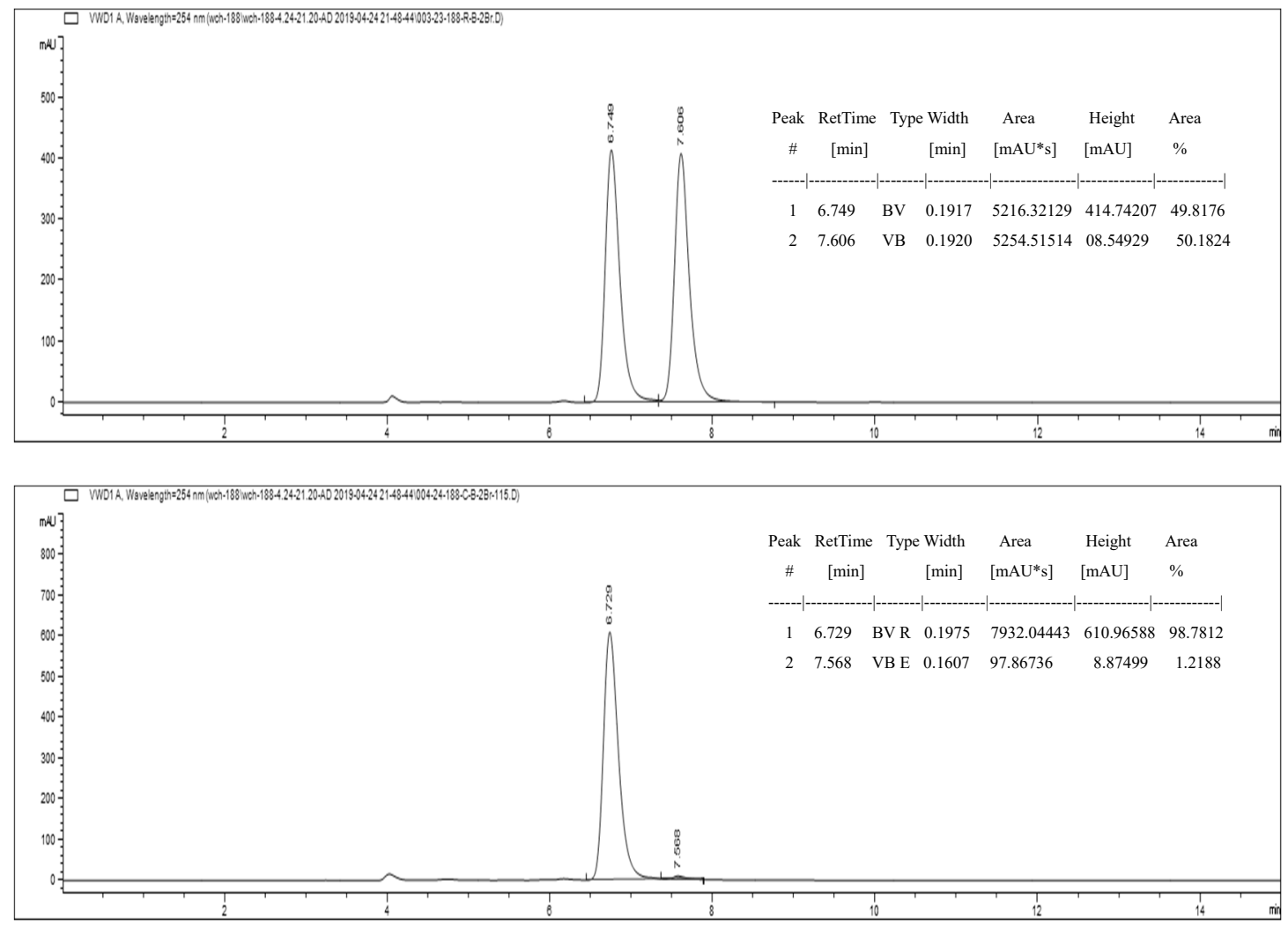
(S)-4-(3,5-bis(trifluoromethyl)benzyl)

1-methyl 2-(3-bromophenyl)-3-phenyl-2,3-dihydro-1H-pyrazole-1,4-dicarboxylate.<smiles>CC(=O)N1C=C(C(=O)OCc2cc(C(F)(F)F)cc(C(F)(F)F)c2)[C@H](c2ccccc2)N1c1cccc(Br)c1</smiles>

Viscous liquid, 96\% yield $(60 \mathrm{mg})$. Purified by flash chromatography $(12 \% \mathrm{EtOAc} / \mathrm{PE}) ;[\alpha]$ ${ }_{\mathrm{D}}^{20}=+164.6\left(c 0.39, \mathrm{CH}_{2} \mathrm{Cl}_{2}\right) ;{ }^{1} \mathrm{H}$ NMR $\left(300 \mathrm{MHz}, \mathrm{CDCl}_{3}\right) \delta 8.02(\mathrm{~s}, 1 \mathrm{H}), 7.86(\mathrm{~d}, J=7.5 \mathrm{~Hz}$, 1H), $7.70(\mathrm{~s}, 2 \mathrm{H}), 7.64(\mathrm{dd}, J=7.9,1.5 \mathrm{~Hz}, 1 \mathrm{H}), 7.56-7.47(\mathrm{~m}, 2 \mathrm{H}), 7.42-7.29(\mathrm{~m}, 5 \mathrm{H})$, $7.17-7.02(\mathrm{~m}, 2 \mathrm{H}), 5.34(\mathrm{~s}, 1 \mathrm{H}), 5.27(\mathrm{~d}, J=13.0 \mathrm{~Hz}, 1 \mathrm{H}), 5.12(\mathrm{~d}, J=13.1 \mathrm{~Hz}, 1 \mathrm{H}), 3.83(\mathrm{~s}$, $3 \mathrm{H}) ;{ }^{13} \mathrm{C} \mathrm{NMR}\left(101 \mathrm{MHz}, \mathrm{CDCl}_{3}\right) \delta 162.7,148.4,145.2,140.0,138.2,136.3,134.4,132.0$ (q, $2 J \mathrm{C}-\mathrm{F}=33.5 \mathrm{~Hz}), 128.4,128.2,128.1,128.0,127.0,126.7,123.1(\mathrm{q}, 1 J \mathrm{C}-\mathrm{F}=272.8 \mathrm{~Hz})$, $122.2(\mathrm{sep}, 3 J \mathrm{C}-\mathrm{F}=3.5 \mathrm{~Hz}), 120.0,117.3,112.8,75.7,64.7,54.3$; IR (film) $v_{\max } 2958,1709$, $1622,1507,1445,1387,1358,1280,1245,1179,1134,1073,1037,884,844 \mathrm{~cm}^{-1}$; HRMS (ESI) calcd for $\mathrm{C}_{27} \mathrm{H}_{20} \mathrm{BrF}_{6} \mathrm{~N}_{2} \mathrm{O}_{4}{ }^{+}[\mathrm{M}+\mathrm{H}]^{+}$629.0505, found 629.0483. HPLC analysis: 91\% ee $($ CHIRALPAK IC, isopropanol/hexane $=10: 90,1.0 \mathrm{~mL} / \mathrm{min}, \mathrm{UV}: 254 \mathrm{~nm}), t_{\mathrm{R}}=7.008 \mathrm{~min}$ (major), $9.593 \min$ (minor).
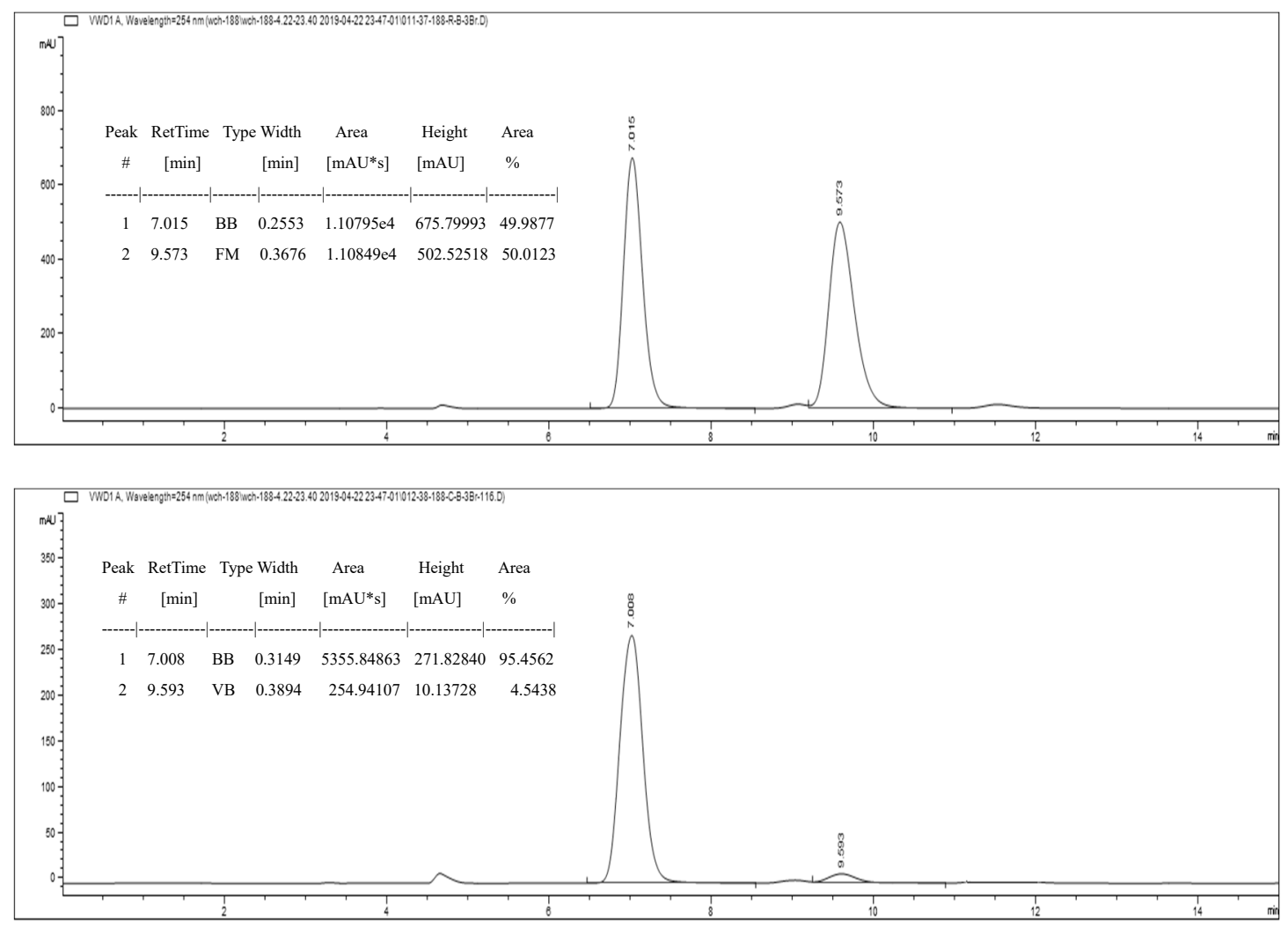
(S)-4-(3,5-bis(trifluoromethyl)benzyl)

1-methyl 2-(4-bromophenyl)-3-phenyl-2,3-dihydro-1H-pyrazole-1,4-dicarboxylate.<smiles>CC(=O)N1C=C(C(=O)OCc2cc(C(F)(F)F)cc(C(F)(F)F)c2)[C@H](c2ccccc2)N1c1ccc(Br)cc1</smiles>

Pale yellow solid, 99\% yield (62 mg). Purified by flash chromatography (12\% EtOAc/PE). $\mathrm{mp}=54-55{ }^{\circ} \mathrm{C} ;[\alpha]{ }^{20}{ }_{\mathrm{D}}=+147.8\left(c 0.53, \mathrm{CH}_{2} \mathrm{Cl}_{2}\right) ;{ }^{1} \mathrm{H}$ NMR $\left(300 \mathrm{MHz}, \mathrm{CDCl}_{3}\right) \delta 7.99(\mathrm{~d}, J$ $=1.1 \mathrm{~Hz}, 1 \mathrm{H}), 7.85(\mathrm{~s}, 1 \mathrm{H}), 7.67(\mathrm{~s}, 2 \mathrm{H}), 7.55-7.31(\mathrm{~m}, 8 \mathrm{H}), 6.99-6.85(\mathrm{~m}, 1 \mathrm{H}), 5.39(\mathrm{~d}, J$ $=1.1 \mathrm{~Hz}, 1 \mathrm{H}), 5.27(\mathrm{~d}, J=13.0 \mathrm{~Hz}, 1 \mathrm{H}), 5.10(\mathrm{~d}, J=13.1 \mathrm{~Hz}, 1 \mathrm{H}), 3.88(\mathrm{~s}, 3 \mathrm{H}) ;{ }^{13} \mathrm{C} \mathrm{NMR}$ $\left(101 \mathrm{MHz}, \mathrm{CDCl}_{3}\right) \delta 162.4,151.8,150.4,140.1,138.1,136.3,132.3,132.0(\mathrm{q}, 3 J \mathrm{C}-\mathrm{F}=33.6$ Hz), 129.0, 128.7, $128.1(\mathrm{q}, 3 J \mathrm{C}-\mathrm{F}=3.7 \mathrm{~Hz}), 126.6,123.1(\mathrm{q}, 1 J \mathrm{C}-\mathrm{F}=273.9 \mathrm{~Hz}), 122.3(\mathrm{sep}$, $3 J \mathrm{C}-\mathrm{F}=3.4 \mathrm{~Hz}), 118.5,116.8,112.8,77.0,64.7,54.4$; IR (film) $v_{\max } 2360,1712,1625,1486$, $1445,1387,1357,1280,1264,1178,1137,1074,1005,970,891,844,735,703,683 \mathrm{~cm}^{-1}$; HRMS (ESI) calcd for $\mathrm{C}_{27} \mathrm{H}_{20} \mathrm{BrF}_{6} \mathrm{~N}_{2} \mathrm{O}_{4}{ }^{+}[\mathrm{M}+\mathrm{H}]^{+}$629.0505, found 629.0473. HPLC analysis: 95\% ee (CHIRALPAK IC, isopropanol/hexane $=10: 90,1.0 \mathrm{~mL} / \mathrm{min}, \mathrm{UV}: 254 \mathrm{~nm}), t_{\mathrm{R}}=$ 6.925 min (major), 8.863 min (minor).
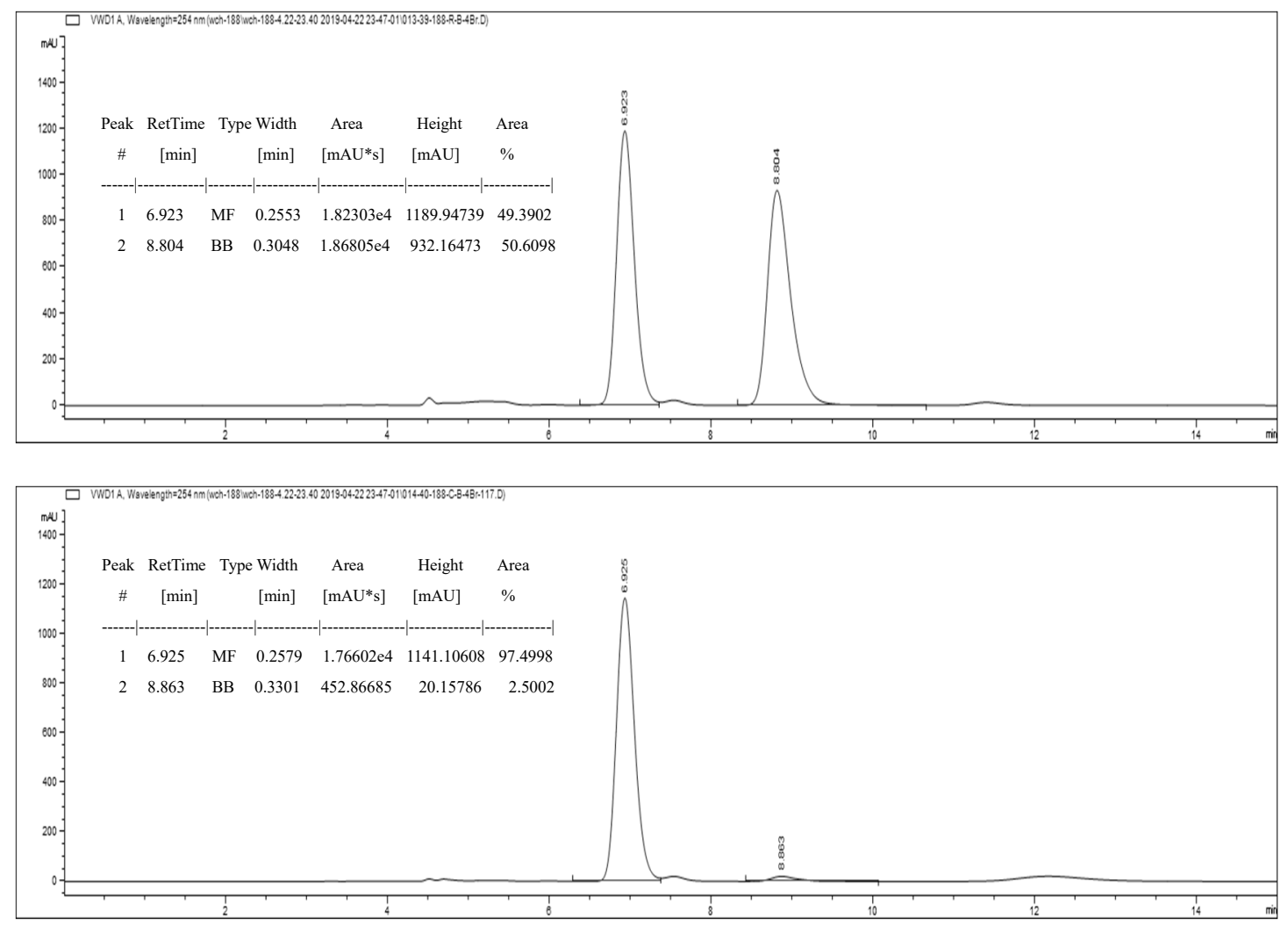
(S)-4-(3,5-bis(trifluoromethyl)benzyl) 1-methyl 3-phenyl-2-(4-(trifluoromethyl)phenyl)2,3-dihydro-1H-pyrazole-1,4-dicarboxylate.<smiles>CC(=O)N1C=C(C(=O)OCc2cc(C(F)(F)F)cc(C(F)(F)F)c2)[C@H](c2ccccc2)N1c1ccc(C(F)(F)F)cc1</smiles>

Viscous liquid, 35\% yield (22 mg). Purified by flash chromatography (12\% EtOAc/PE); $[\alpha]$ ${ }_{\mathrm{D}}^{20}=+60.5\left(c 0.17, \mathrm{CH}_{2} \mathrm{Cl}_{2}\right) ;{ }^{1} \mathrm{H} \mathrm{NMR}\left(300 \mathrm{MHz}, \mathrm{CDCl}_{3}\right) \delta 7.97(\mathrm{~d}, J=1.1 \mathrm{~Hz}, 1 \mathrm{H}), 7.85(\mathrm{~s}$, 1H), $7.71-7.65(\mathrm{~m}, 2 \mathrm{H}), 7.63-7.57(\mathrm{~m}, 2 \mathrm{H}), 7.52-7.36(\mathrm{~m}, 5 \mathrm{H}), 7.09$ (d, J = 8.2 Hz, 2H), $5.51(\mathrm{~d}, J=1.1 \mathrm{~Hz}, 1 \mathrm{H}), 5.29(\mathrm{~d}, J=13.0 \mathrm{~Hz}, 1 \mathrm{H}), 5.11(\mathrm{~d}, J=13.0 \mathrm{~Hz}, 1 \mathrm{H}), 3.92(\mathrm{~s}, 3 \mathrm{H}),{ }^{13} \mathrm{C}$ NMR $\left(126 \mathrm{MHz}, \mathrm{CDCl}_{3}\right) \delta 161.1,152.8,151.0,138.8,137.0,135.2,131.0(\mathrm{q}, 2 J \mathrm{C}-\mathrm{F}=33.6$ $\mathrm{Hz}), 128.0,127.7,127.1(\mathrm{q}, 3 J \mathrm{C}-\mathrm{F}=3.9 \mathrm{~Hz}), 125.53(\mathrm{q}, 3 J \mathrm{C}-\mathrm{F}=2.4 \mathrm{~Hz}), 125.50,124.6(\mathrm{q}$, $2 J \mathrm{C}-\mathrm{F}=32.9 \mathrm{~Hz}), 123.1(\mathrm{q}, 1 J \mathrm{C}-\mathrm{F}=271.5 \mathrm{~Hz}), 122.0(\mathrm{q}, 1 J \mathrm{C}-\mathrm{F}=273.0 \mathrm{~Hz}), 121.2(\mathrm{sep}$, $3 J \mathrm{C}-\mathrm{F}=3.6 \mathrm{~Hz}$ ), 115.1, 112.5, 75.4, 63.7, 53.5; IR (film) $v_{\max } 2963,2360,2342,1716,1615$, 1507, 1446, 1327, 1278, 1260, 1179, 1135, 1070, 886, 844, 764, $750 \mathrm{~cm}^{-1}$; HRMS (ESI) calcd for $\mathrm{C}_{28} \mathrm{H}_{20} \mathrm{~F}_{9} \mathrm{~N}_{2} \mathrm{O}_{4}^{+}[\mathrm{M}+\mathrm{H}]^{+}$619.1274, found 619.1271. HPLC analysis: $92 \%$ ee $($ CHIRALPAK IC, isopropanol/hexane $=10: 90,1.0 \mathrm{~mL} / \mathrm{min}, \mathrm{UV}: 254 \mathrm{~nm}), t_{\mathrm{R}}=5.144 \mathrm{~min}$ (major), $6.122 \mathrm{~min}$ (minor).
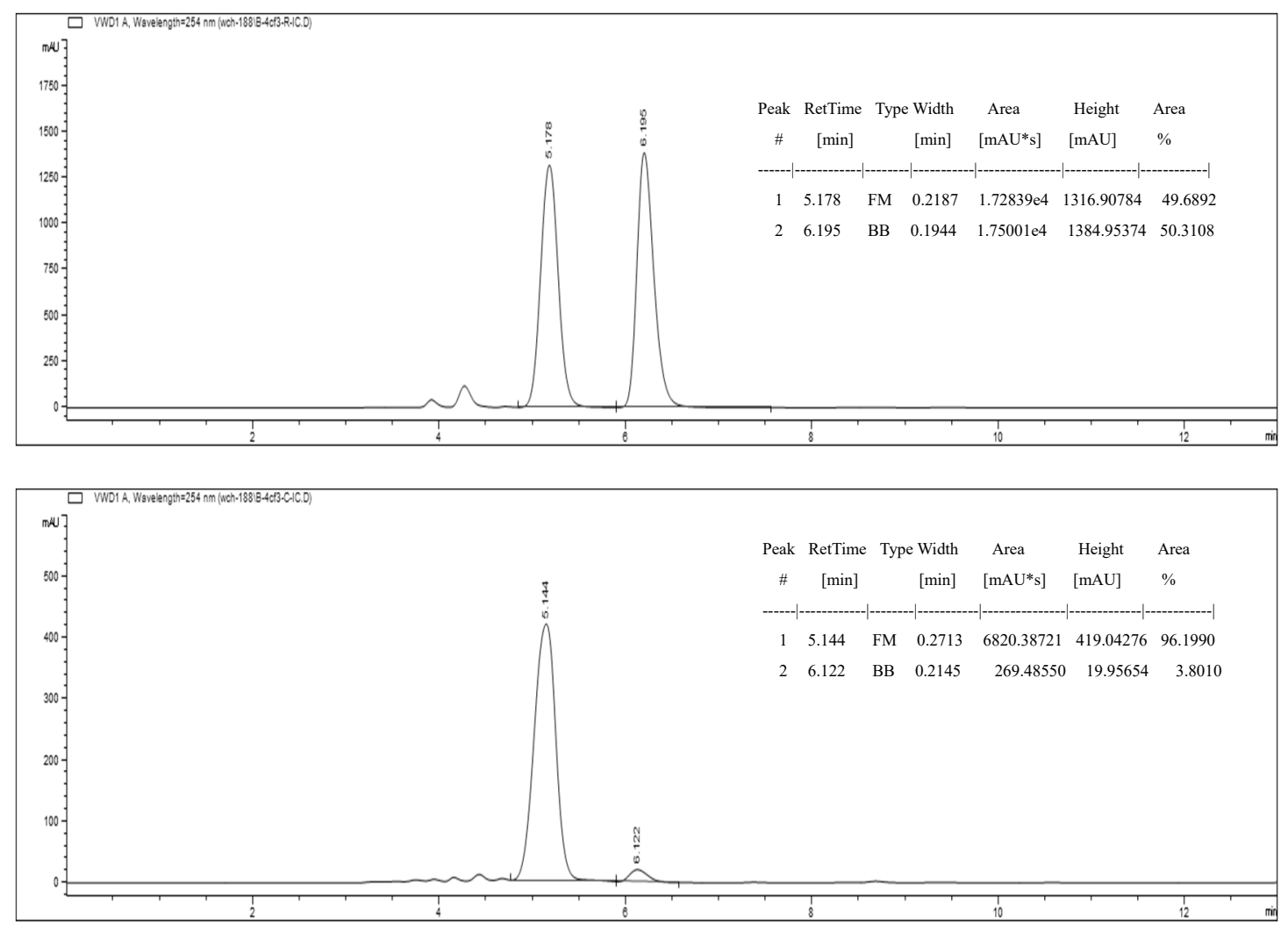


\section{H-pyrazole-1,4-dicarboxylate}<smiles>CC(=O)N1C=C(C(=O)OCc2cc(C(F)(F)F)cc(C(F)(F)F)c2)[C@H](c2ccccc2)[C@H]1c1ccccc1C</smiles>

Pale yellow solid, 99\% yield (56 mg). Purified by flash chromatography (12\% EtOAc/PE). $\mathrm{mp}=48-49^{\circ} \mathrm{C} ;\left[\alpha{ }^{20}{ }_{\mathrm{D}}=+201.7\left(c 0.58, \mathrm{CH}_{2} \mathrm{Cl}_{2}\right) ;{ }^{1} \mathrm{H}\right.$ NMR $\left(300 \mathrm{MHz}, \mathrm{CDCl}_{3}\right) \delta 8.03(\mathrm{~s}$, 1H), $7.85(\mathrm{~s}, 1 \mathrm{H}), 7.70(\mathrm{~s}, 2 \mathrm{H}), 7.50-7.31(\mathrm{~m}, 5 \mathrm{H}), 7.27-7.08(\mathrm{~m}, 4 \mathrm{H}), 5.32-5.07(\mathrm{~m}, 3 \mathrm{H})$, $3.82(\mathrm{~s}, 3 \mathrm{H}), 2.16(\mathrm{~s}, 3 \mathrm{H}) ;{ }^{13} \mathrm{C}$ NMR $\left(101 \mathrm{MHz}, \mathrm{CDCl}_{3}\right) \delta 162.9,149.4,140.7,138.3,136.5$, $132.6,131.9(\mathrm{q}, 2 J \mathrm{C}-\mathrm{F}=33.5 \mathrm{~Hz}), 131.6,128.6,128.2(\mathrm{q}, 3 J \mathrm{C}-\mathrm{F}=4.0 \mathrm{~Hz}), 128.1,126.7$, 126.5, 125.9, $123.1(\mathrm{q}, 1 J \mathrm{C}-\mathrm{F}=272.7 \mathrm{~Hz}), 122.2(\mathrm{sep}, 3 J \mathrm{C}-\mathrm{F}=3.7 \mathrm{~Hz}), 118.3,112.0,75.8$, 64.6, 54.1, 18.9; IR (film) $v_{\max }$ 2959, 2330, 1708, 1620, 1490, 1446, 1387, 1358, 1279, 1241, 1179, 1134, 1072, 1031, 970, 885, 844, 804, 751, $683 \mathrm{~cm}^{-1}$; HRMS (ESI) calcd for $\mathrm{C}_{28} \mathrm{H}_{23} \mathrm{~F}_{6} \mathrm{~N}_{2} \mathrm{O}_{4}{ }^{+}[\mathrm{M}+\mathrm{H}]^{+}$565.1557, found 565.1544. HPLC analysis: 95\% ee (CHIRALPAK IC, isopropanol/hexane $=10: 90,1.0 \mathrm{~mL} / \mathrm{min}, \mathrm{UV}: 254 \mathrm{~nm}$ ), $t_{\mathrm{R}}=6.598 \mathrm{~min}$ (major), $8.942 \mathrm{~min}$ (minor).
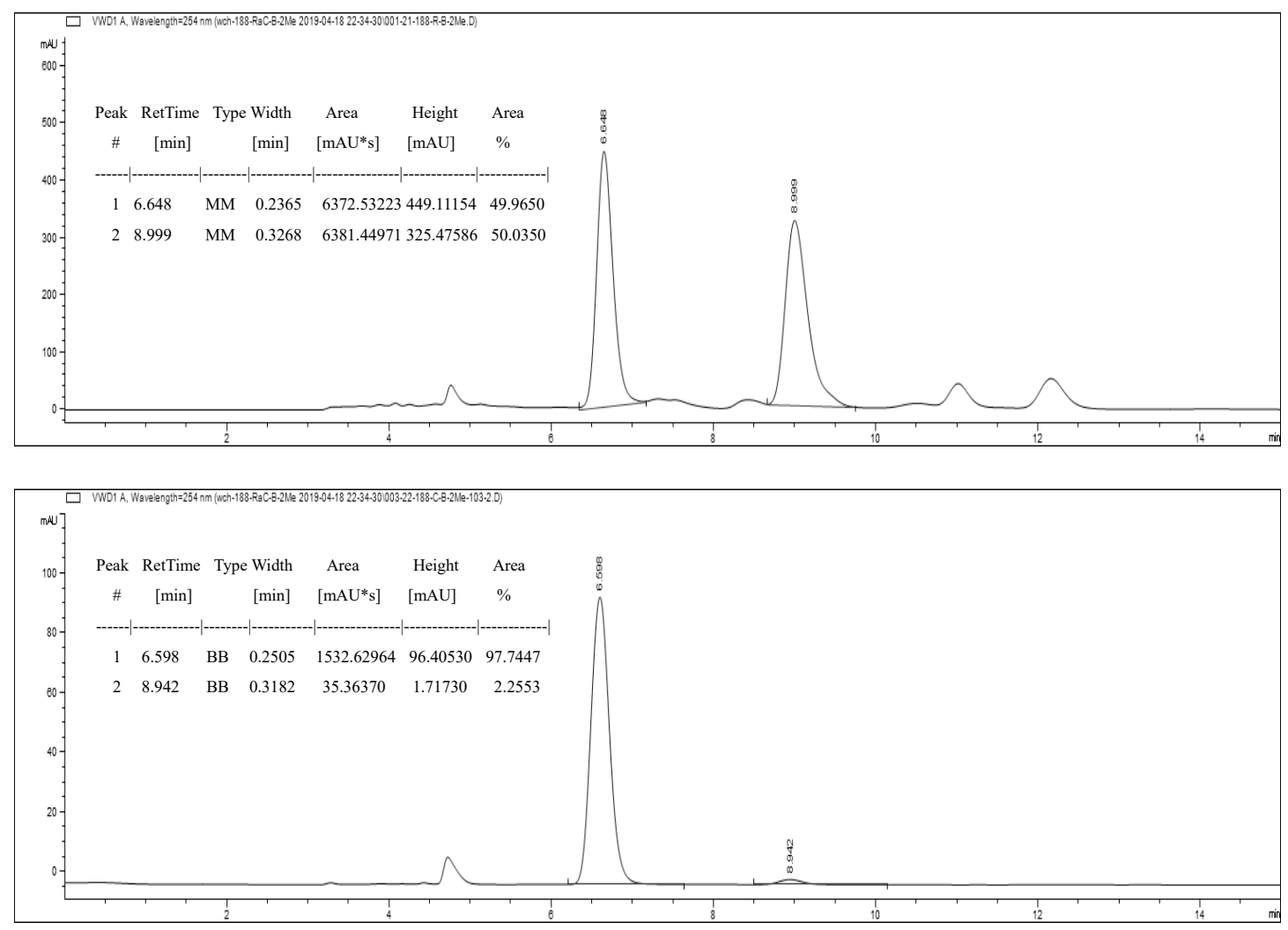
(S)-4-(3,5-bis(trifluoromethyl)benzyl)

3-phenyl-2-(m-tolyl)-2,3-dihydro-1H-pyrazole-1,4-dicarboxylate<smiles>CC(=O)N1C=C(C(=O)OCc2cc(C(F)(F)F)cc(C(F)(F)F)c2)[C@H](c2ccccc2)N1c1cccc(C)c1</smiles>

Pale yellow solid, 96\% yield (54 mg). Purified by flash chromatography (12\% EtOAc/PE). $\mathrm{mp}=104-105{ }^{\circ} \mathrm{C} ;[\alpha]^{20}{ }_{\mathrm{D}}=+109.8\left(c 0.49, \mathrm{CH}_{2} \mathrm{Cl}_{2}\right) ;{ }^{1} \mathrm{H}$ NMR $\left(300 \mathrm{MHz}, \mathrm{CDCl}_{3}\right) \delta 8.03(\mathrm{~d}$, $J=1.0 \mathrm{~Hz}, 1 \mathrm{H}), 7.85(\mathrm{~s}, 1 \mathrm{H}), 7.68(\mathrm{~s}, 2 \mathrm{H}), 7.52-7.34(\mathrm{~m}, 5 \mathrm{H}), 7.25(\mathrm{dd}, J=8.9,7.5 \mathrm{~Hz}, 1 \mathrm{H})$, $7.03-6.78(\mathrm{~m}, 3 \mathrm{H}), 5.44(\mathrm{~d}, J=1.1 \mathrm{~Hz}, 1 \mathrm{H}), 5.27(\mathrm{~d}, J=13.1 \mathrm{~Hz}, 1 \mathrm{H}), 5.10(\mathrm{~d}, J=13.1 \mathrm{~Hz}$, 1H), $3.88(\mathrm{~s}, 3 \mathrm{H}), 2.37(\mathrm{~s}, 3 \mathrm{H}) ;{ }^{13} \mathrm{C}$ NMR $\left(101 \mathrm{MHz}, \mathrm{CDCl}_{3}\right) \delta$ 162.6, 152.0, 151.4, 140.6, $139.4,138.2,136.5,131.9(\mathrm{q}, 2 J \mathrm{C}-\mathrm{F}=33.4 \mathrm{~Hz}), 129.2,128.9,128.5,128.1(\mathrm{q}, 3 J \mathrm{C}-\mathrm{F}=3.6$ Hz), 126.6, 125.1, $123.1(\mathrm{q}, 1 J \mathrm{C}-\mathrm{F}=273.8 \mathrm{~Hz}), 122.2(\mathrm{sep}, 3 J \mathrm{C}-\mathrm{F}=3.6 \mathrm{~Hz}), 117.5,113.9$, 112.8, 77.3, 64.6, 54.3, 21.6; IR (film) $v_{\max } 3055,2359,1709$, 1624, 1507, 1446, 1387, 1358, $1279,1232,1177,1137,1074,975,894,843,764,733,703,683 \mathrm{~cm}^{-1}$; HRMS (ESI) calcd for $\mathrm{C}_{28} \mathrm{H}_{23} \mathrm{~F}_{6} \mathrm{~N}_{2} \mathrm{O}_{4}{ }^{+}[\mathrm{M}+\mathrm{H}]^{+}$565.1557, found 565.1544. HPLC analysis: 98\% ee (CHIRALPAK $\mathrm{ID}$, isopropanol/hexane $=10: 90,1.0 \mathrm{~mL} / \mathrm{min}, \mathrm{UV}: 254 \mathrm{~nm}$ ), $t_{\mathrm{R}}=6.318 \mathrm{~min}$ (major), 8.225 $\min ($ minor).
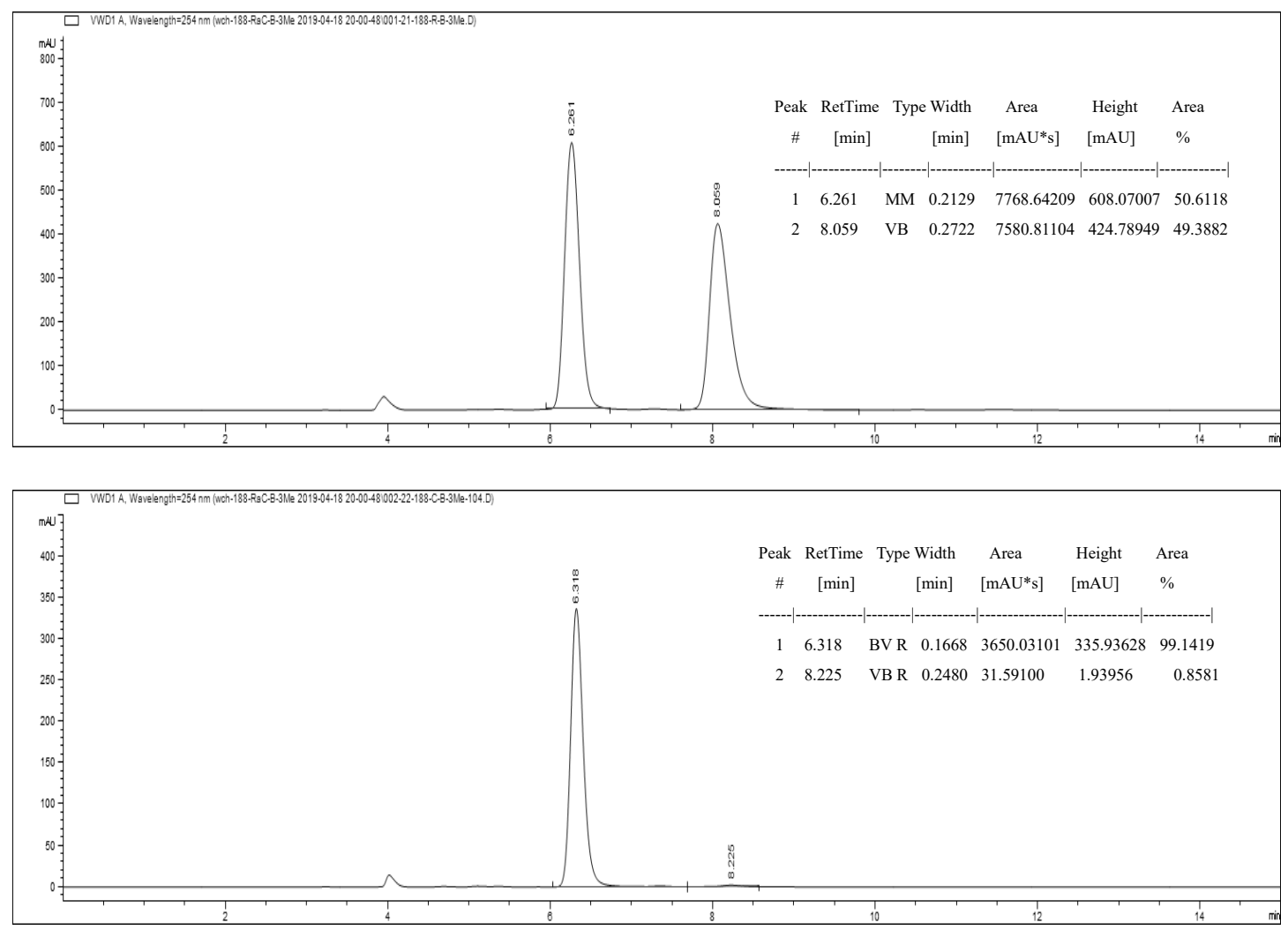


\section{H-pyrazole-1,4-dicarboxylate}<smiles>CC(=O)N1C=C(C(=O)OCc2cc(C(F)(F)F)cc(C(F)(F)F)c2)[C@H](c2ccccc2)[C@H]1c1ccc(C)cc1</smiles>

Pale yellow solid, 99\% yield (56 mg). Purified by flash chromatography (12\% EtOAc/PE). $\mathrm{mp}=107-108{ }^{\circ} \mathrm{C} ;[\alpha]^{20}{ }_{\mathrm{D}}=+131.4\left(c 0.57, \mathrm{CH}_{2} \mathrm{Cl}_{2}\right) ;{ }^{1} \mathrm{H} \mathrm{NMR}\left(300 \mathrm{MHz}, \mathrm{CDCl}_{3}\right) \delta 8.04(\mathrm{~d}$, $J=1.1 \mathrm{~Hz}, 1 \mathrm{H}), 7.85(\mathrm{~s}, 1 \mathrm{H}), 7.67$ (s, 2H), $7.49-7.33$ (m, 5H), $7.22-7.12(\mathrm{~m}, 2 \mathrm{H}), 7.06-$ $6.91(\mathrm{~m}, 2 \mathrm{H}), 5.39$ (d, $J=1.1 \mathrm{~Hz}, 1 \mathrm{H}), 5.27(\mathrm{~d}, J=13.1 \mathrm{~Hz}, 1 \mathrm{H}), 5.09(\mathrm{~d}, J=13.1 \mathrm{~Hz}, 1 \mathrm{H})$, $3.86(\mathrm{~s}, 3 \mathrm{H}), 2.35$ (s, 3H); ${ }^{13} \mathrm{C}$ NMR $\left(101 \mathrm{MHz}, \mathrm{CDCl}_{3}\right) \delta 162.7,151.8,149.2,140.6,138.3$, 136.5, 134.0, 131.9 (q, 2JC-F = 33.4 Hz), 129.9, 128.9, 128.5, 128.1 (q, 3JC-F = 3.9 Hz), $126.7,123.1(\mathrm{q}, 1 J \mathrm{C}-\mathrm{F}=273.8 \mathrm{~Hz}), 122.2(\mathrm{sep}, 3 J \mathrm{C}-\mathrm{F}=3.6 \mathrm{~Hz}), 117.1,112.4,77.3,64.6$, 54.2, 20.7; IR (film) $v_{\max } 2959,2360,1710,1624,1508,1446,1387,1358,1280,1247,1177$, 1136, 1073, 970, 911, 884, 844, 814, $765 \mathrm{~cm}^{-1}$; HRMS (ESI) calcd for $\mathrm{C}_{28} \mathrm{H}_{23} \mathrm{~F}_{6} \mathrm{~N}_{2} \mathrm{O}_{4}{ }^{+}$ $[\mathrm{M}+\mathrm{H}]^{+}$565.1557, found 565.1545. HPLC analysis: $96 \%$ ee (CHIRALPAK IC, isopropanol/hexane $=10: 90,1.0 \mathrm{~mL} / \mathrm{min}, \mathrm{UV}: 254 \mathrm{~nm}$ ), $t_{\mathrm{R}}=8.589 \mathrm{~min}$ (major), $13.489 \mathrm{~min}$ (minor).
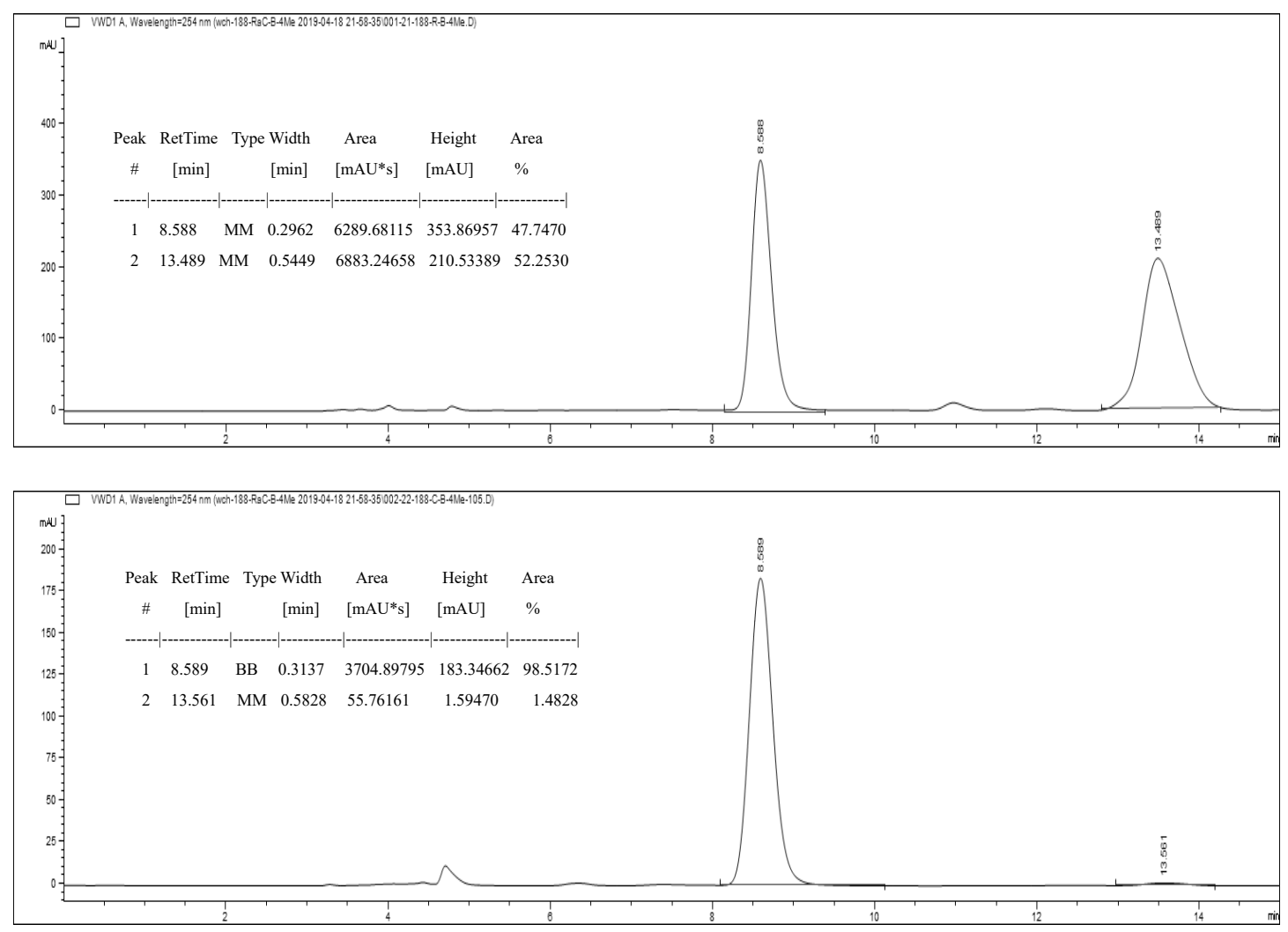
(S)-4-(3,5-bis(trifluoromethyl)benzyl) 1-methyl 2-(2,4-dimethylphenyl)-3-phenyl-2,3-dihydro-1H-pyrazole-1,4-dicarboxylate (3at).<smiles>CC(=O)N1C=C(C(=O)OCc2cc(C(F)(F)F)cc(C(F)(F)F)c2)[C@H](c2ccccc2)[C@H]1c1ccc(C)cc1C</smiles>

Pale yellow solid, 92\% yield $(53 \mathrm{mg})$. Purified by flash chromatography (12\% EtOAc/PE). $\mathrm{mp}=53-54{ }^{\circ} \mathrm{C} ;[\alpha]^{20}{ }_{\mathrm{D}}=+281.5\left(c 0.44, \mathrm{CH}_{2} \mathrm{Cl}_{2}\right) ;{ }^{1} \mathrm{H}$ NMR $\left(300 \mathrm{MHz}, \mathrm{CDCl}_{3}\right) \delta 8.02(\mathrm{~s}$, 1H), $7.85(\mathrm{~s}, 1 \mathrm{H}), 7.69(\mathrm{~s}, 2 \mathrm{H}), 7.51-7.30(\mathrm{~m}, 5 \mathrm{H}), 7.10-6.97(\mathrm{~m}, 3 \mathrm{H}), 5.36-5.04(\mathrm{~m}, 3 \mathrm{H})$, $3.82(\mathrm{~s}, 3 \mathrm{H}), 2.32(\mathrm{~s}, 3 \mathrm{H}), 2.12(\mathrm{~s}, 3 \mathrm{H}) ;{ }^{13} \mathrm{C} \mathrm{NMR}\left(101 \mathrm{MHz}, \mathrm{CDCl}_{3}\right) \delta 162.9,147.0,140.8$, $138.3,136.6,135.6,132.6,132.2,131.9(\mathrm{q}, 2 J \mathrm{C}-\mathrm{F}=33.5 \mathrm{~Hz}), 128.5,128.1(\mathrm{q}, 3 J \mathrm{C}-\mathrm{F}=3.4$ Hz), 128.0, 127.3, 126.6, 123.1 (q, 1JC-F = 272.8 Hz), $122.2(\mathrm{sep}, 3 J \mathrm{C}-\mathrm{F}=3.6 \mathrm{~Hz}), 118.4$, 111.9, 76.0, 64.5, 54.0, 20.8, 18.7; IR (film) $v_{\max } 2958,2358,1708,1620,1497,1447,1387$, 1359, 1279, 1234, 1180, 1135, 1071, 970, 908, 884, 844, 814, $765 \mathrm{~cm}^{-1}$; HRMS (ESI) calcd for $\mathrm{C}_{29} \mathrm{H}_{25} \mathrm{~F}_{6} \mathrm{~N}_{2} \mathrm{O}_{4}^{+}[\mathrm{M}+\mathrm{H}]^{+}$579.1713, found 579.1697. HPLC analysis: $96 \%$ ee $($ CHIRALPAK IC, isopropanol/hexane $=3: 97,1.0 \mathrm{~mL} / \mathrm{min}, \mathrm{UV}: 254 \mathrm{~nm}), t_{\mathrm{R}}=12.789 \mathrm{~min}$ (major), $22.722 \mathrm{~min}$ (minor).
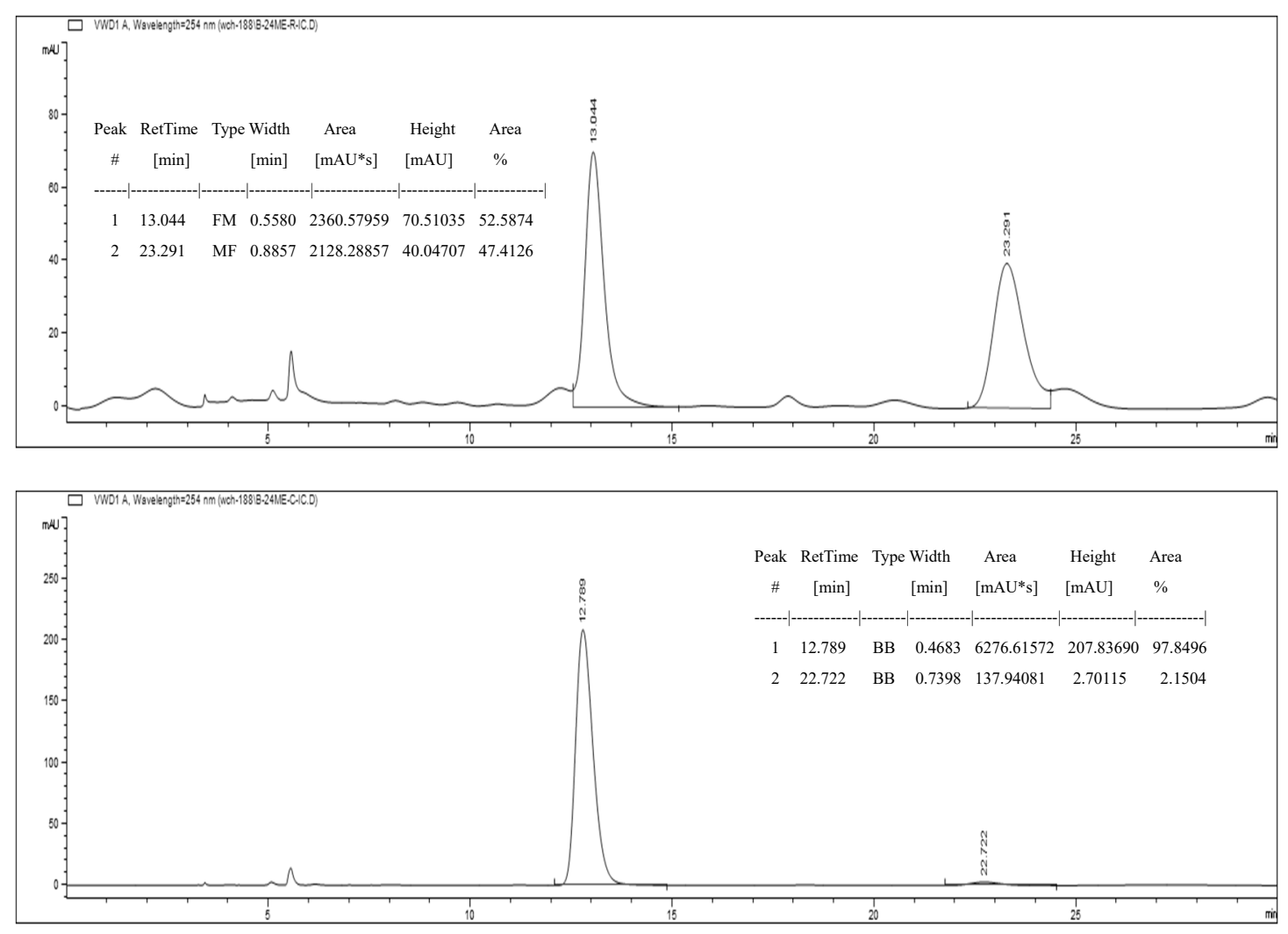
<smiles>CCc1ccc(N2[C@H](c3ccccc3)C(C(=O)OCc3cc(C(F)(F)F)cc(C(F)(F)F)c3)=CN2C(C)=O)cc1</smiles>

White solid, $99 \%$ yield $(57 \mathrm{mg})$. Purified by flash chromatography (12\% EtOAc/PE). $\mathrm{mp}=79$ $-80{ }^{\circ} \mathrm{C} ;[\alpha]{ }_{\mathrm{D}}^{20}=+138.9\left(c 0.41, \mathrm{CH}_{2} \mathrm{Cl}_{2}\right) ;{ }^{1} \mathrm{H}$ NMR $\left(300 \mathrm{MHz}, \mathrm{CDCl}_{3}\right) \delta 8.03(\mathrm{~d}, J=1.0 \mathrm{~Hz}$, 1H), $7.89-7.78(\mathrm{~m}, 1 \mathrm{H}), 7.71-7.62(\mathrm{~m}, 2 \mathrm{H}), 7.49-7.34(\mathrm{~m}, 5 \mathrm{H}), 7.24-7.12(\mathrm{~m}, 2 \mathrm{H}), 7.08$ $-6.93(\mathrm{~m}, 2 \mathrm{H}), 5.40(\mathrm{~d}, J=1.1 \mathrm{~Hz}, 1 \mathrm{H}), 5.26(\mathrm{~d}, J=13.1 \mathrm{~Hz}, 1 \mathrm{H}), 5.09(\mathrm{~d}, J=13.1 \mathrm{~Hz}, 1 \mathrm{H})$, $3.86(\mathrm{~s}, 3 \mathrm{H}), 2.65(\mathrm{q}, J=7.6 \mathrm{~Hz}, 2 \mathrm{H}), 1.24(\mathrm{t}, J=7.6 \mathrm{~Hz}, 3 \mathrm{H}) ;{ }^{13} \mathrm{C} \mathrm{NMR}\left(126 \mathrm{MHz}, \mathrm{CDCl}_{3}\right) \delta$ 161.6, 150.8, 148.2, 139.5, 139.3, 137.2, 135.5, 130.9 (q, 2JC-F = 33.5 Hz), 127.9, 127.7, $127.4,127.0(\mathrm{q}, 3 J \mathrm{C}-\mathrm{F}=4.3 \mathrm{~Hz}), 125.6,122.0(\mathrm{q}, 1 J \mathrm{C}-\mathrm{F}=272.8 \mathrm{~Hz}), 121.2(\mathrm{sep}, 3 J \mathrm{C}-\mathrm{F}=$ $3.7 \mathrm{~Hz}$ ), 116.0, 111.4, 76.2, 63.5, 53.2, 27.1, 14.6; IR (film) $v_{\max } 2963,1708,1623,1508$, $1444,1386,1357,1278,1246,1173,1131,1071,969,885,844,802,750 \mathrm{~cm}^{-1}$; HRMS (ESI) calcd for $\mathrm{C}_{29} \mathrm{H}_{25} \mathrm{~F}_{6} \mathrm{~N}_{2} \mathrm{O}_{4}{ }^{+}[\mathrm{M}+\mathrm{H}]^{+}$579.1713, found 579.1701. HPLC analysis: 97\% ee $($ CHIRALPAK IC, isopropanol/hexane $=10: 90,1.0 \mathrm{~mL} / \mathrm{min}, \mathrm{UV}: 254 \mathrm{~nm}), t_{\mathrm{R}}=7.864 \mathrm{~min}$ (major), 13.059 min (minor).
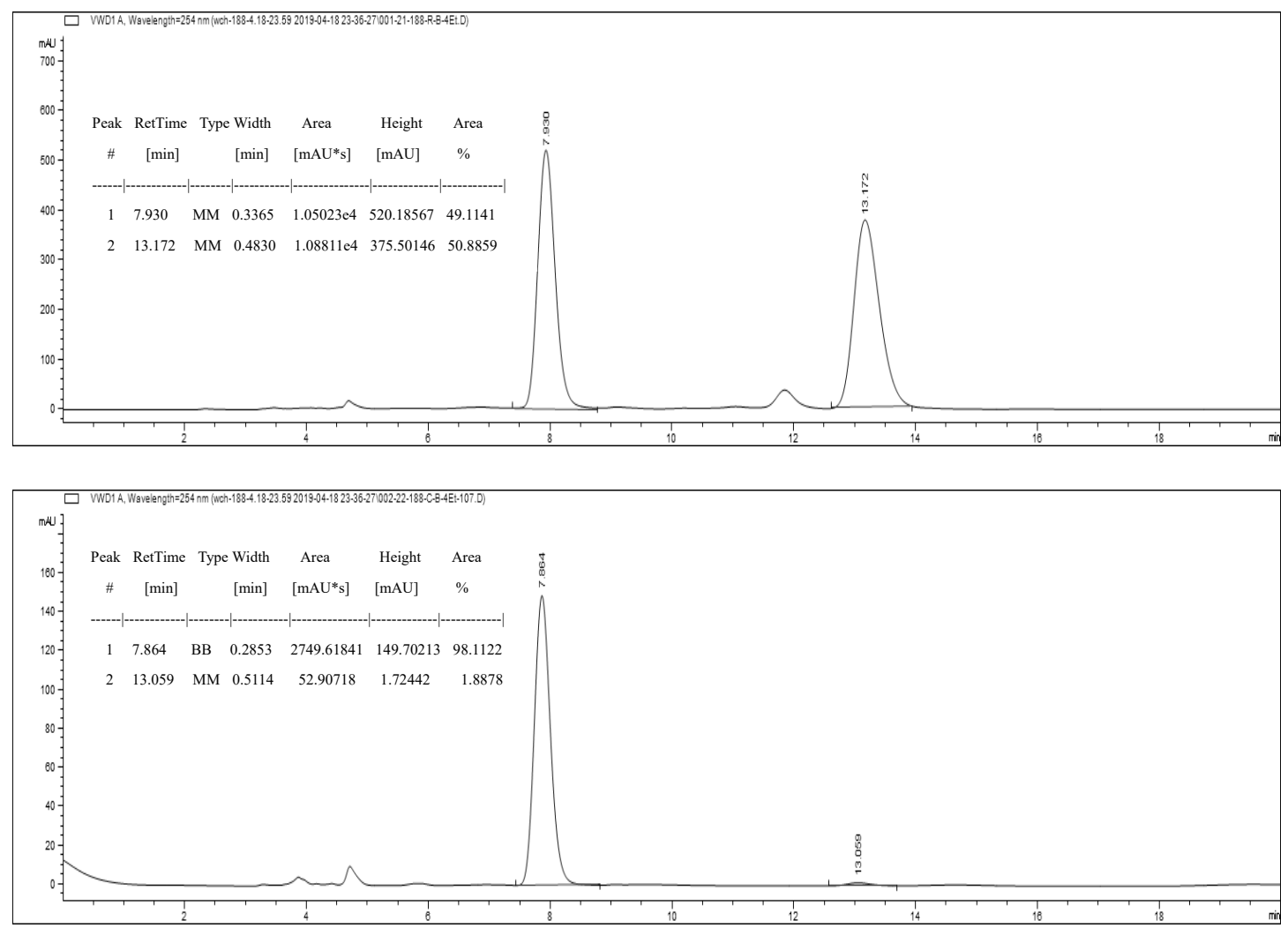
(S)-4-(3,5-bis(trifluoromethyl)benzyl)

2-(3-methoxyphenyl)-3-phenyl-2,3-dihydro-1H-pyrazole-1,4-dicarboxylate<smiles>COc1cccc(N2C(C(C)=O)=CN(c3ccccc3)[C@H]2c2ccccc2)c1</smiles>

Viscous liquid, 97\% yield (56 mg). Purified by flash chromatography (12\% EtOAc/PE); $[\alpha]$ ${ }_{\mathrm{D}}^{20}=+98.5\left(c 0.53, \mathrm{CH}_{2} \mathrm{Cl}_{2}\right) ;{ }^{1} \mathrm{H} \mathrm{NMR}\left(300 \mathrm{MHz}, \mathrm{CDCl}_{3}\right) \delta 8.01(\mathrm{~d}, J=1.1 \mathrm{~Hz}, 1 \mathrm{H}), 7.85(\mathrm{~s}$, 1H), 7.67 (d, $J=1.6 \mathrm{~Hz}, 2 \mathrm{H}), 7.41$ (dddd, $J=13.3,8.3,6.6,3.2 \mathrm{~Hz}, 5 \mathrm{H}), 7.31-7.21$ (m, 1H), $6.73-6.56(\mathrm{~m}, 3 \mathrm{H}), 5.47(\mathrm{~d}, J=1.1 \mathrm{~Hz}, 1 \mathrm{H}), 5.27(\mathrm{~d}, J=13.0 \mathrm{~Hz}, 1 \mathrm{H}), 5.09(\mathrm{~d}, J=13.1 \mathrm{~Hz}$, 1H), $3.87(\mathrm{~s}, 3 \mathrm{H}), 3.81(\mathrm{~s}, 3 \mathrm{H}) ;{ }^{13} \mathrm{C}$ NMR $\left(101 \mathrm{MHz}, \mathrm{CDCl}_{3}\right) \delta$ 162.5, 160.5, 152.9, 151.9, $140.4,138.2,136.5,131.9(\mathrm{q}, 2 J \mathrm{C}-\mathrm{F}=33.5 \mathrm{~Hz}), 130.2,129.0,128.5,128.1(\mathrm{q}, 3 J \mathrm{C}-\mathrm{F}=3.7$ Hz), 126.6, $123.1(\mathrm{q}, 1 J \mathrm{C}-\mathrm{F}=272.9 \mathrm{~Hz}), 122.2(\mathrm{sep}, 3 J \mathrm{C}-\mathrm{F}=3.5 \mathrm{~Hz}), 112.9,109.2,109.1$, 103.4, 77.0, 64.6, 55.3, 54.3; IR (film) $v_{\max } 2959,2360,1711,1624,1603,1489,1445,1386$, 1357, 1279, 1237, 1177, 1136, 1073, 985, 886, 843, $765 \mathrm{~cm}^{-1}$; HRMS (ESI) calcd for $\mathrm{C}_{28} \mathrm{H}_{23} \mathrm{~F}_{6} \mathrm{~N}_{2} \mathrm{O}_{5}{ }^{+}[\mathrm{M}+\mathrm{H}]^{+} 581.1506$ found 581.1494. HPLC analysis: 98\% ee (CHIRALPAK ID, isopropanol/hexane $=10: 90,1.0 \mathrm{~mL} / \mathrm{min}, \mathrm{UV}: 254 \mathrm{~nm}$ ), $t_{\mathrm{R}}=11.648 \mathrm{~min}$ (major), $13.946 \mathrm{~min}$ (minor).
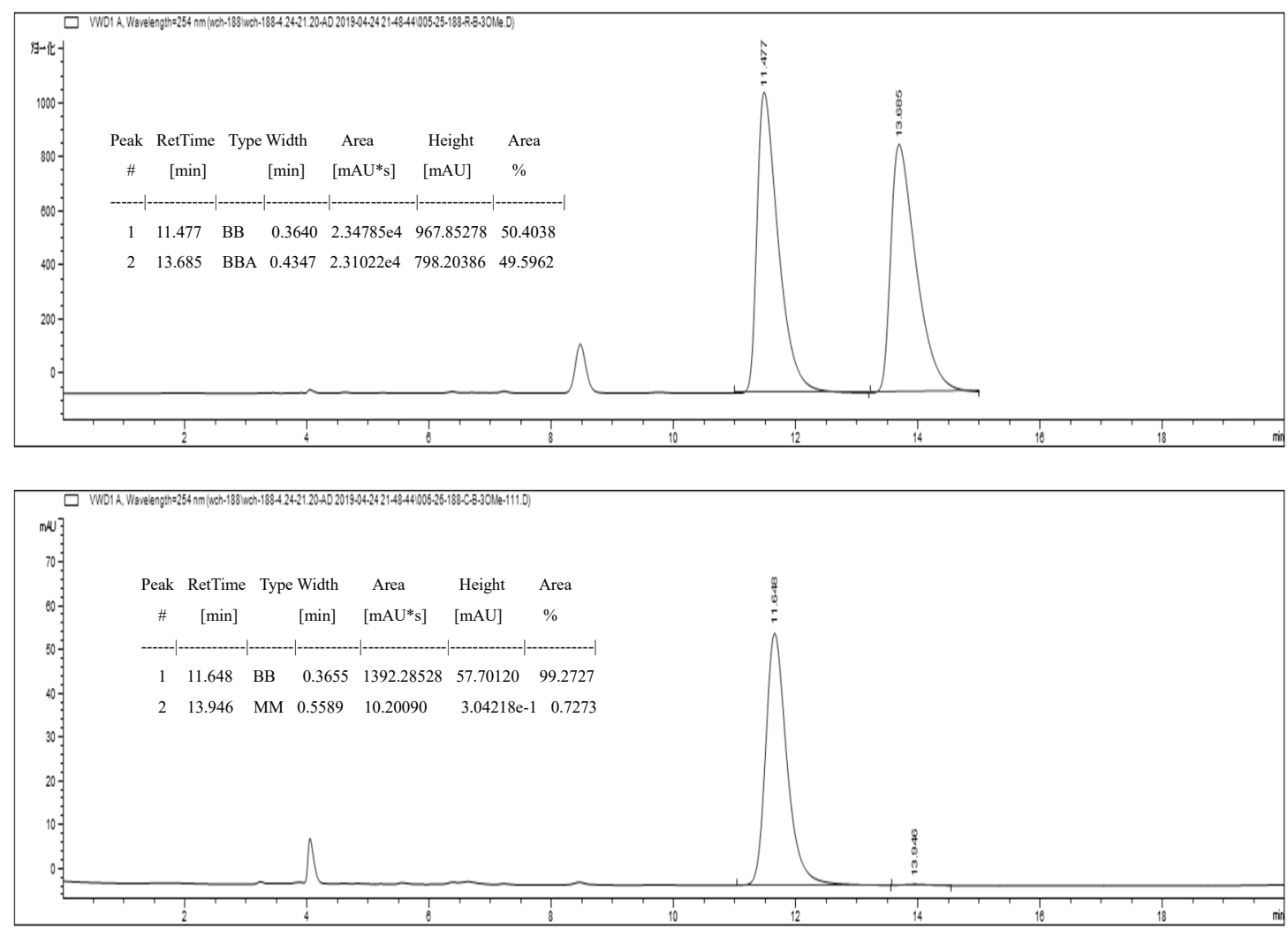
(S)-4-(3,5-bis(trifluoromethyl)benzyl) 1-methyl 2-(4-methoxyphenyl)-3-phenyl-2,3dihydro-1H-pyrazole-1,4-dicarboxylate<smiles>COc1ccc(N2C(C(=O)OCc3cc(C(F)(F)F)cc(C(F)(F)F)c3)=CN(C(C)=O)[C@H]2c2ccccc2)cc1</smiles>

White solid, $98 \%$ yield $(57 \mathrm{mg})$. Purified by flash chromatography $(12 \% \mathrm{EtOAc} / \mathrm{PE}) . \mathrm{mp}=51$ $-52{ }^{\circ} \mathrm{C} ;[\alpha]^{20}{ }_{\mathrm{D}}=+84.6\left(c 0.60, \mathrm{CH}_{2} \mathrm{Cl}_{2}\right) ;{ }^{1} \mathrm{H}$ NMR $\left(300 \mathrm{MHz}, \mathrm{CDCl}_{3}\right) \delta 8.04(\mathrm{~d}, J=1.1 \mathrm{~Hz}$, 1H), $7.84(\mathrm{~s}, 1 \mathrm{H}), 7.70-7.62(\mathrm{~m}, 2 \mathrm{H}), 7.39$ (tdd, $J=8.8,6.4,5.2 \mathrm{~Hz}, 5 \mathrm{H}), 7.09-6.98(\mathrm{~m}$, 2H), $6.96-6.84(\mathrm{~m}, 2 \mathrm{H}), 5.32(\mathrm{~d}, J=1.1 \mathrm{~Hz}, 1 \mathrm{H}), 5.26(\mathrm{~d}, J=13.1 \mathrm{~Hz}, 1 \mathrm{H}), 5.09(\mathrm{~d}, J=13.1$ $\mathrm{Hz}, 1 \mathrm{H}), 3.84(\mathrm{~s}, 3 \mathrm{H}), 3.81$ (s, 3H); ${ }^{13} \mathrm{C} \mathrm{NMR}\left(101 \mathrm{MHz}, \mathrm{CDCl}_{3}\right) \delta 162.7,156.9,151.7,144.8$, 140.6, 138.2, 136.6, 131.9 (q, 2JC-F = 33.6 Hz), 128.9, 128.4, 128.0 (q, 3JC-F = 3.9 Hz), 126.7, $123.1(\mathrm{q}, 1 J \mathrm{C}-\mathrm{F}=271.6 \mathrm{~Hz}), 122.2(\mathrm{sep}, 3 J \mathrm{C}-\mathrm{F}=3.8 \mathrm{~Hz}), 119.2,114.6,111.9,77.5$, 64.5, 55.5, 54.2; IR (film) $v_{\max }$ 3094, 2358, 1710, 1622, 1468, 1447, 1387, 1359, 1280, 1240, 1180, 1136, 1072, 1032, 969, 884, 843, $765 \mathrm{~cm}^{-1}$; HRMS (ESI) calcd for $\mathrm{C}_{28} \mathrm{H}_{23} \mathrm{~F}_{6} \mathrm{~N}_{2} \mathrm{O}_{5}{ }^{+}$ $[\mathrm{M}+\mathrm{H}]^{+}$581.1506, found 581.1489. HPLC analysis: $96 \%$ ee (CHIRALPAK ID, isopropanol/hexane $=10: 90,1.0 \mathrm{~mL} / \mathrm{min}, \mathrm{UV}: 254 \mathrm{~nm}$ ), $t_{\mathrm{R}}=13.087 \mathrm{~min}$ (major), $16.528 \mathrm{~min}$ (minor).
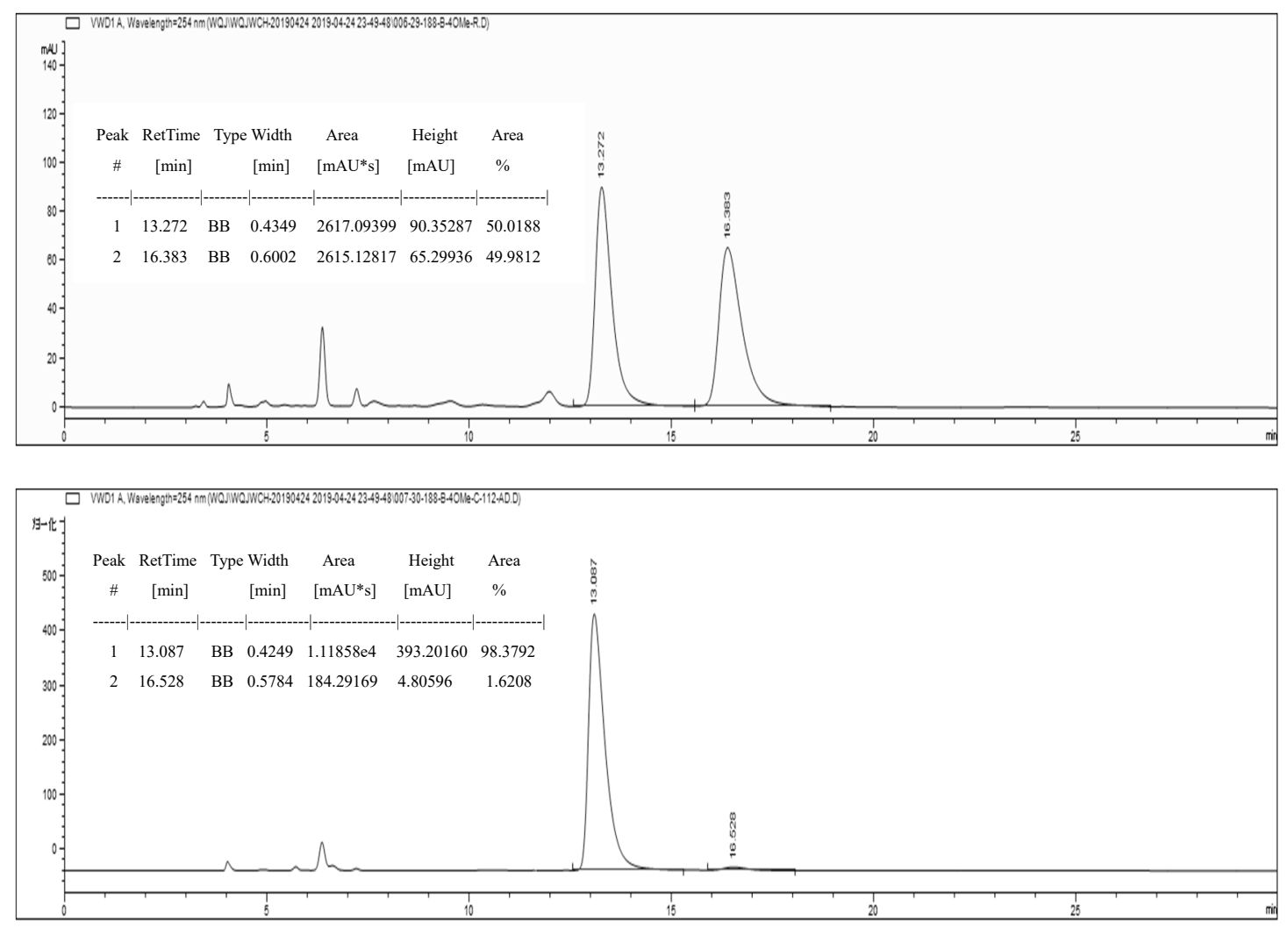
(S)-4-(3,5-bis(trifluoromethyl)benzyl) 1-methyl 2-(naphthalen-2-yl)-3-phenyl-2,3-dihydro-1H-pyrazole-1,4-dicarboxylate (3ar).<smiles>CC(=O)N1C=C(C(=O)OCc2cc(C(F)(F)F)cc(C(F)(F)F)c2)C(c2ccccc2)N1c1ccc2ccccc2c1</smiles>

Yellow solid, 99\% yield (59 mg). Purified by flash chromatography (12\% EtOAc/PE). mp = $63-64{ }^{\circ} \mathrm{C} ;[\alpha]{ }^{20}{ }_{\mathrm{D}}=+196.1\left(c 0.54, \mathrm{CH}_{2} \mathrm{Cl}_{2}\right) ;{ }^{1} \mathrm{H}$ NMR $\left(300 \mathrm{MHz}, \mathrm{CDCl}_{3}\right) \delta 8.11(\mathrm{~d}, J=1.1$ $\mathrm{Hz}, 1 \mathrm{H}), 7.90-7.75(\mathrm{~m}, 4 \mathrm{H}), 7.67$ (s, 2H), $7.56-7.36$ (m, 8H), 7.28 (dd, J=8.8, 2.4 Hz, 1H), $5.55(\mathrm{~d}, J=1.0 \mathrm{~Hz}, 1 \mathrm{H}), 5.27$ (d, $J=13.1 \mathrm{~Hz}, 1 \mathrm{H}), 5.09$ (d, $J=13.1 \mathrm{~Hz}, 1 \mathrm{H}), 3.88$ (s, 3H); ${ }^{13} \mathrm{C}$ NMR $\left(101 \mathrm{MHz}, \mathrm{CDCl}_{3}\right) \delta 162.6,152.0,148.7,140.4,138.2,136.6,133.7,131.9$ (q, $2 J \mathrm{C}-\mathrm{F}=33.4 \mathrm{~Hz}), 130.9,129.7,129.0,128.6,128.1(\mathrm{q}, 3 J \mathrm{C}-\mathrm{F}=3.5 \mathrm{~Hz}), 127.7,127.4,126.8$, 126.7, 125.1, $123.1(\mathrm{q}, 1 J \mathrm{C}-\mathrm{F}=272.8 \mathrm{~Hz}), 122.3(\mathrm{sep}, 3 J \mathrm{C}-\mathrm{F}=3.8 \mathrm{~Hz}), 118.1,112.8,112.6$, 76.9, 64.6, 54.4; IR (film) $v_{\max }$ 2959, 2360, 1710, 1623, 1600, 1508, 1445, 1387, 1357, 1279, $1255,1238,1174,1135,1073,982,884,844,750 \mathrm{~cm}^{-1}$; HRMS (ESI) calcd for $\mathrm{C}_{31} \mathrm{H}_{23} \mathrm{~F}_{6} \mathrm{~N}_{2} \mathrm{O}_{4}{ }^{+}[\mathrm{M}+\mathrm{H}]^{+}$601.1557, found 601.1541. HPLC analysis: 99\% ee (CHIRALPAK IC, isopropanol/hexane $=10: 90,1.0 \mathrm{~mL} / \mathrm{min}, \mathrm{UV}: 254 \mathrm{~nm}$ ), $t_{\mathrm{R}}=9.379 \mathrm{~min}$ (major), $13.474 \mathrm{~min}$ (minor).
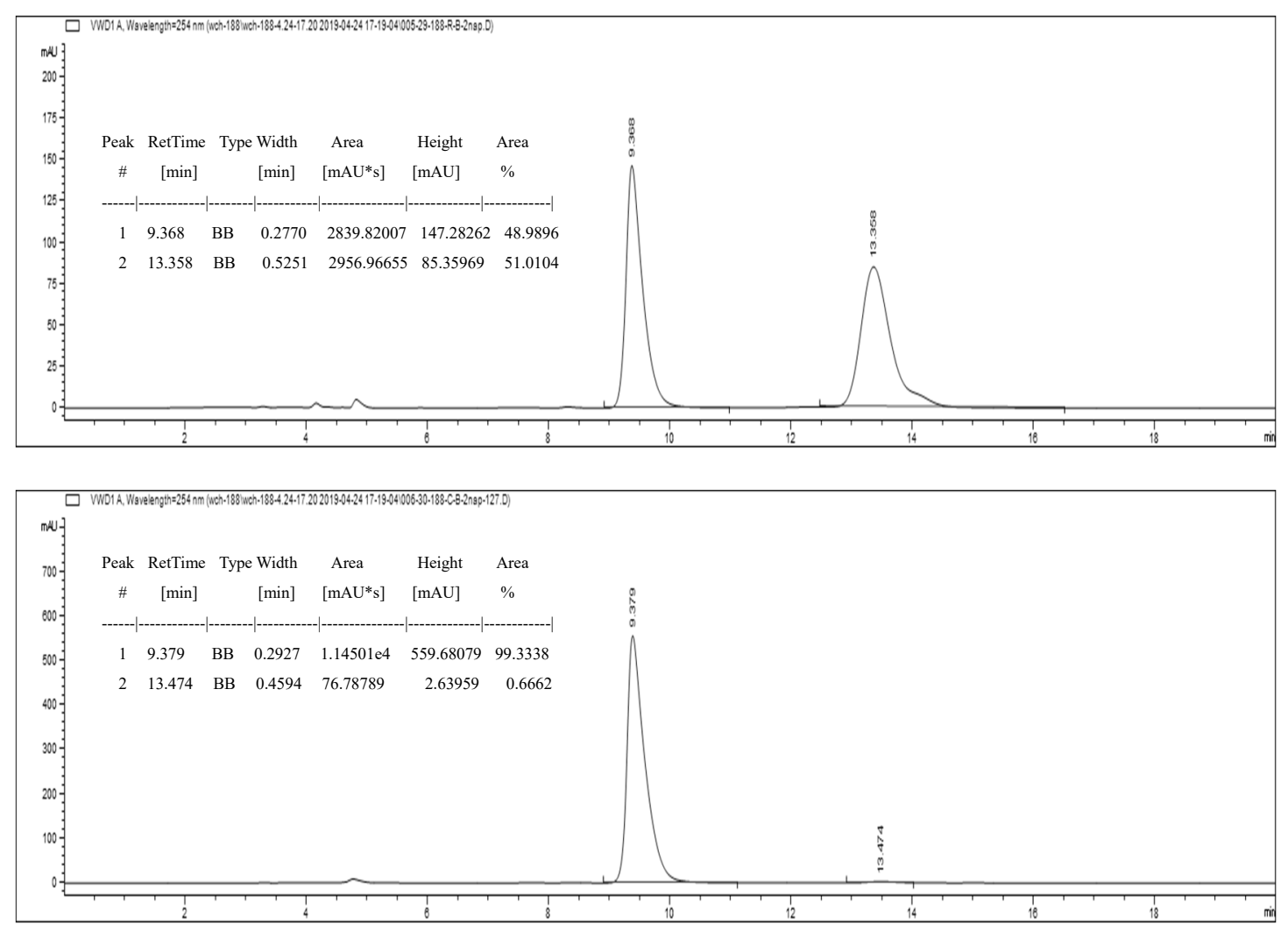
(S)-4-(3,5-bis(trifluoromethyl)benzyl) 1-methyl 2-(6-bromonaphthalen-2-yl)-3-phenyl2,3-dihydro-1H-pyrazole-1,4-dicarboxylate.<smiles>CC(=O)N1C=C(C(=O)OCc2cc(C(F)(F)F)cc(C(F)(F)F)c2)[C@H](c2ccccc2)N1c1ccc2cc(Br)ccc2c1</smiles>

Yellow solid, 99\% yield $(67 \mathrm{mg})$. Purified by flash chromatography $(12 \%$ EtOAc/PE). $\mathrm{mp}=$ $52-53{ }^{\circ} \mathrm{C} ;[\alpha]{ }^{20}{ }_{\mathrm{D}}=+187.2\left(c 0.69, \mathrm{CH}_{2} \mathrm{Cl}_{2}\right) ;{ }^{1} \mathrm{H}$ NMR $\left(300 \mathrm{MHz}, \mathrm{CDCl}_{3}\right) \delta 8.05(\mathrm{~d}, J=1.1$ $\mathrm{Hz}, 1 \mathrm{H}), 7.94$ (d, $J=1.9 \mathrm{~Hz}, 1 \mathrm{H}), 7.82$ (s, 1H), 7.73 (d, $J=8.9 \mathrm{~Hz}, 1 \mathrm{H}), 7.69-7.59$ (m, 3H), $7.56-7.47(\mathrm{~m}, 3 \mathrm{H}), 7.46-7.36(\mathrm{~m}, 3 \mathrm{H}), 7.32(\mathrm{~d}, J=2.3 \mathrm{~Hz}, 1 \mathrm{H}), 7.28-7.23(\mathrm{~m}, 1 \mathrm{H}), 5.53$ $(\mathrm{d}, J=1.1 \mathrm{~Hz}, 1 \mathrm{H}), 5.25(\mathrm{~d}, J=13.1 \mathrm{~Hz}, 1 \mathrm{H}), 5.07(\mathrm{~d}, J=13.1 \mathrm{~Hz}, 1 \mathrm{H}), 3.87(\mathrm{~s}, 3 \mathrm{H}) ;{ }^{13} \mathrm{C}$ $\operatorname{NMR}\left(75 \mathrm{MHz}, \mathrm{CDCl}_{3}\right) \delta 162.1,151.6,148.7,139.9,137.8,136.2,131.8,131.6(\mathrm{q}, 2 J \mathrm{C}-\mathrm{F}=$ $33.5 \mathrm{~Hz}), 131.4,129.7,129.3,128.6,128.4,128.3,127.7$ (q, 3JC-F=3.4 Hz), 126.3, 122.7 (q, $1 J \mathrm{C}-\mathrm{F}=271.1 \mathrm{~Hz}), 121.8(\mathrm{sep}, 3 J \mathrm{C}-\mathrm{F}=3.8 \mathrm{~Hz}), 118.6,118.3,112.5,112.2,76.3,64.3,54.0$; IR (film) $v_{\max } 2959,1711,1622,1590,1497,1445,1386,1357,1280,1252,1236,1179,1136$, 1073, 1030, 985, 882, 843, $765 \mathrm{~cm}^{-1}$; HRMS (ESI) calcd for $\mathrm{C}_{31} \mathrm{H}_{22} \mathrm{BrF}_{6} \mathrm{~N}_{2} \mathrm{O}_{4}{ }^{+}[\mathrm{M}+\mathrm{H}]^{+}$ 679.0662, found 679.0677. HPLC analysis: 96\% ee (CHIRALPAK ID, isopropanol/hexane = 5:95, $1.0 \mathrm{~mL} / \mathrm{min}, \mathrm{UV}: 254 \mathrm{~nm}$ ), $t_{\mathrm{R}}=14.582 \mathrm{~min}$ (major), $16.800 \mathrm{~min}$ (minor).
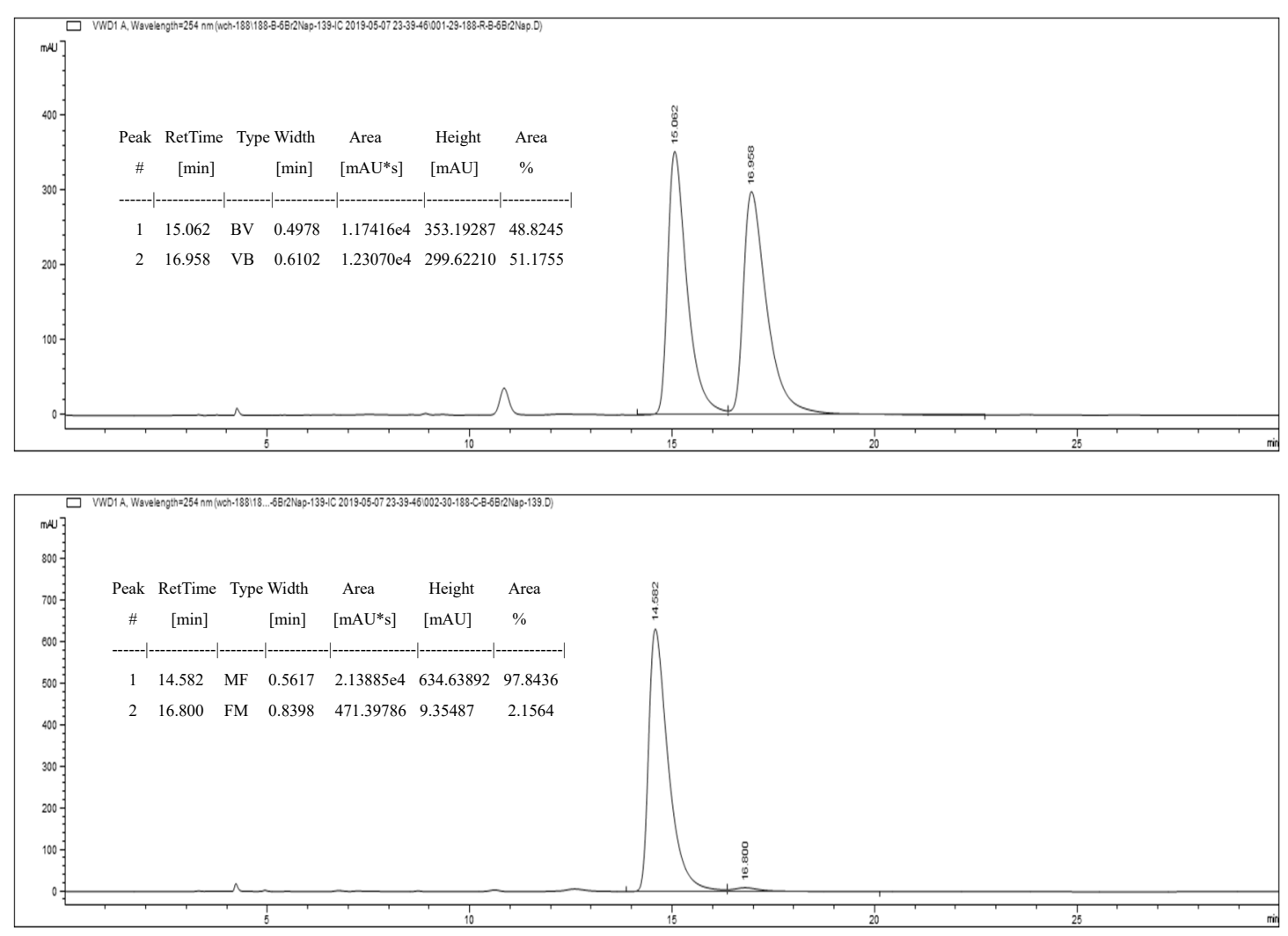
(S)-4-(3,5-bis(trifluoromethyl)benzyl) 1-methyl $\quad$-2-(4'-chloro-[1,1'-biphenyl]-2-yl)-3phenyl-2,3-dihydro-1H-pyrazole-1,4-dicarboxylate<smiles>CC(=O)N1C=C(C(=O)OCc2cc(C(F)(F)F)cc(C(F)(F)F)c2)[C@@H](c2ccccc2)N1c1ccccc1-c1ccc(Cl)cc1</smiles>

Viscous liquid, 43\% yield (28 mg). Purified by flash chromatography (12\% EtOAc/PE); $[\alpha]$ ${ }_{\mathrm{D}}^{20}=+74.5\left(c 0.26, \mathrm{CH}_{2} \mathrm{Cl}_{2}\right) ;{ }^{1} \mathrm{H}$ NMR $\left(300 \mathrm{MHz}, \mathrm{CDCl}_{3}\right) 8.03(\mathrm{~d}, J=1.1 \mathrm{~Hz}, 1 \mathrm{H}), 7.84(\mathrm{~s}$, 1H), $7.66(\mathrm{~s}, 2 \mathrm{H}), 7.52-7.35(\mathrm{~m}, 10 \mathrm{H}), 7.32$ (dd, $J=1.7,1.0 \mathrm{~Hz}, 1 \mathrm{H}), 7.22-7.15(\mathrm{~m}, 1 \mathrm{H})$, 7.04 (ddd, $J=8.0,2.4,1.0 \mathrm{~Hz}, 1 \mathrm{H}), 5.49$ (d, $J=1.1 \mathrm{~Hz}, 1 \mathrm{H}), 5.33-5.04(\mathrm{~m}, 2 \mathrm{H}), 3.89(\mathrm{~s}$, $3 \mathrm{H}) ;{ }^{13} \mathrm{C}$ NMR (126 MHz, $\left.\mathrm{CDCl}_{3}\right)$ 161.5, 150.9, 140.4, 139.3, 138.2, 137.1, 135.4, 132.7, $130.9(\mathrm{q}, 2 J \mathrm{C}-\mathrm{F}=33.5 \mathrm{~Hz}), 128.8,127.97,127.95,127.6,127.4,127.1(\mathrm{q}, 3 J \mathrm{C}-\mathrm{F}=3.4 \mathrm{~Hz})$, 125.6, $122.0(\mathrm{q}, 1 J \mathrm{C}-\mathrm{F}=273.3 \mathrm{~Hz}), 121.9,121.2(\mathrm{sep}, 3 J \mathrm{C}-\mathrm{F}=3.7 \mathrm{~Hz}), 114.9,114.5,111.9$, 76.1, 63.6, 53.4; IR (film) $v_{\max } 2961,2360,1712,1623,1475,1444,1387,1356,1278,1260$, $1176,1133,1092,1013,883,790,750 \mathrm{~cm}^{-1}$; HRMS (ESI) calcd for $\mathrm{C}_{27} \mathrm{H}_{19} \mathrm{~F}_{8} \mathrm{~N}_{2} \mathrm{O}_{4}{ }^{+}[\mathrm{M}+\mathrm{H}]^{+}$ 587.1212, found 587.1202. HPLC analysis: 96\% ee (CHIRALPAK IC, isopropanol/hexane = 10:90, $1.0 \mathrm{~mL} / \mathrm{min}, \mathrm{UV}: 254 \mathrm{~nm}$ ), $t_{\mathrm{R}}=6.823 \mathrm{~min}$ (major), $9.630 \mathrm{~min}$ (minor).
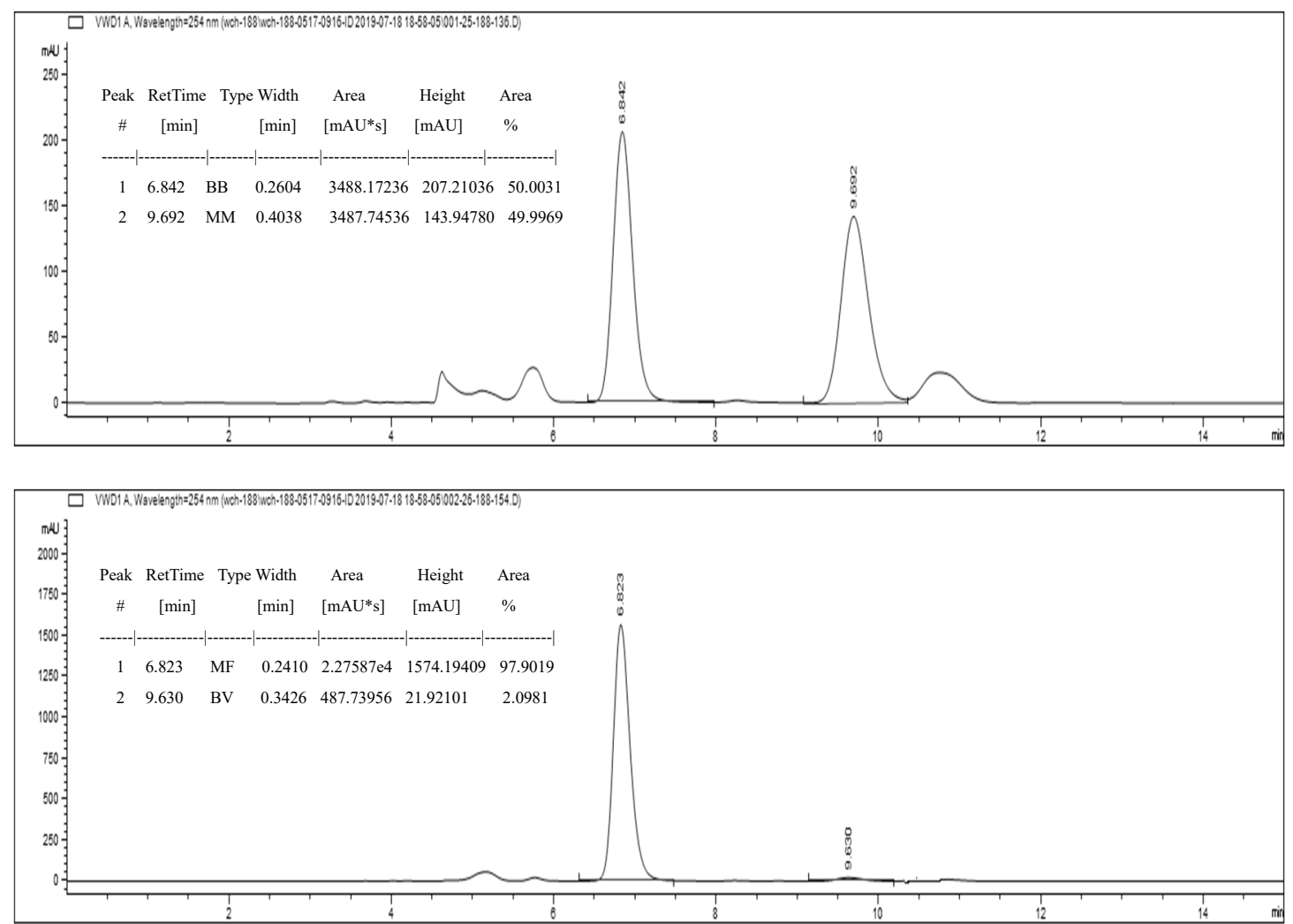
(3R)-dimethyl 2-(2-bromophenyl)-3-phenylpyrazolidine-1,4-dicarboxylate

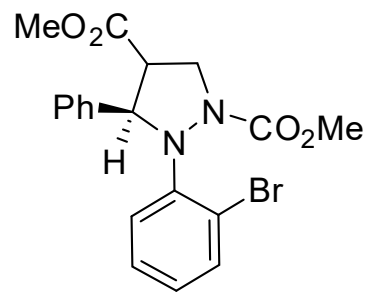

5

Viscous liquid, $67 \%$ yield $(28 \mathrm{mg})$. Purified by flash chromatography (12\% EtOAc/PE); $[\alpha]$ ${ }_{\mathrm{D}}^{20}=-23.5\left(c 0.098, \mathrm{CH}_{2} \mathrm{Cl}_{2}\right) ;{ }^{1} \mathrm{H}$ NMR $\left(300 \mathrm{MHz}, \mathrm{CDCl}_{3}\right) \delta 7.55-7.31(\mathrm{~m}, 5 \mathrm{H}), 7.12-7.03$ (m, 3H), $6.76(\mathrm{dq}, J=6.8,2.4 \mathrm{~Hz}, 1 \mathrm{H}), 5.26-5.10(\mathrm{~m}, 1 \mathrm{H}), 4.50(\mathrm{dd}, J=11.3,7.7 \mathrm{~Hz}, 1 \mathrm{H})$, $3.86(\mathrm{~s}, 3 \mathrm{H}), 3.72(\mathrm{~s}, 3 \mathrm{H}), 3.57(\mathrm{dd}, J=11.3,9.5 \mathrm{~Hz}, 1 \mathrm{H}), 3.43-3.32(\mathrm{~m}, 1 \mathrm{H}) ;{ }^{13} \mathrm{C}$ NMR $(126$ $\left.\mathrm{MHz}, \mathrm{CDCl}_{3}\right) \delta 169.8,150.6,139.6,129.4,128.2,128.1,127.0,124.7,124.6,123.1,122.0$, 115.7, 111.6, 71.4, 54.2, 52.8, 51.6, 47.9; HRMS (ESI) calcd for $\mathrm{C}_{19} \mathrm{H}_{20} \mathrm{BrN}_{2} \mathrm{O}_{4}{ }^{+}[\mathrm{M}+\mathrm{H}]^{+}$ 419.0601, found 419.0604. HPLC analysis: 96\% ee (CHIRALPAK IC, isopropanol/hexane = 10:90, $1.0 \mathrm{~mL} / \mathrm{min}, \mathrm{UV}: 254 \mathrm{~nm}$ ), $t_{\mathrm{R}}=14.830 \mathrm{~min}$ (minor), $19.231 \mathrm{~min}$ (major).
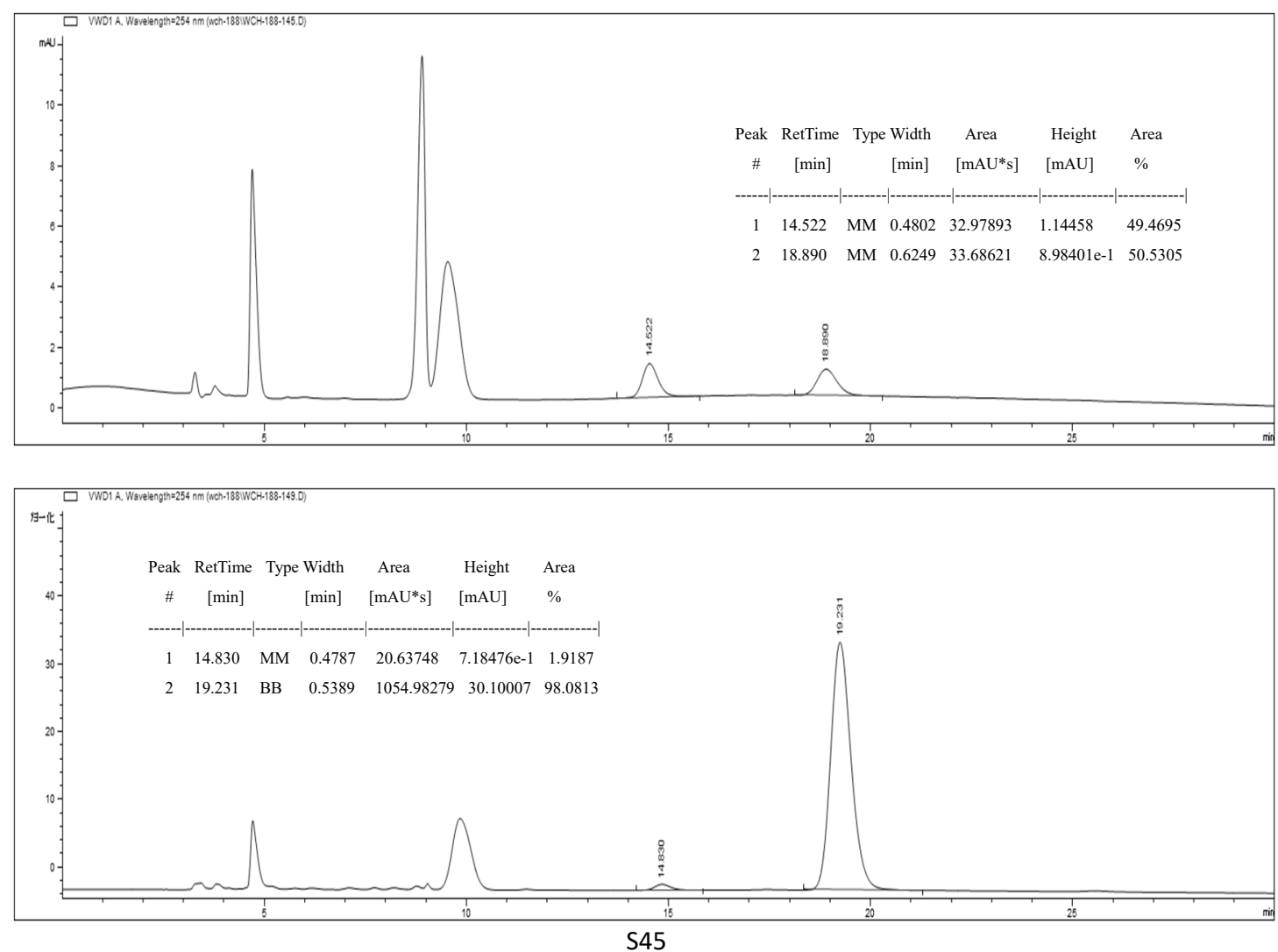
(3R)-4-(3,5-bis(trifluoromethyl)benzyl) 1-methyl-2-(2-bromophenyl)-3-phenyl pyrazolidine-1,4-dicarboxylate<smiles>CC(=O)N1CC(C(=O)OCc2cc(C(F)(F)F)cc(C(F)(F)F)c2)[C@@H](c2ccccc2)N1c1ccccc1Br</smiles>

Viscous liquid, 37\% yield (23 mg). Purified by flash chromatography (12\% EtOAc/PE); $[\alpha]$ ${ }_{\mathrm{D}}^{20}=--19.2\left(c 0.213, \mathrm{CH}_{2} \mathrm{Cl}_{2}\right) ;{ }^{1} \mathrm{H}$ NMR $\left(300 \mathrm{MHz}, \mathrm{CDCl}_{3}\right) \delta 7.93-7.75(\mathrm{~m}, 3 \mathrm{H}), 7.46-$ $7.31(\mathrm{~m}, 5 \mathrm{H}), 7.22(\mathrm{t}, J=2.1 \mathrm{~Hz}, 1 \mathrm{H}), 7.05-6.87(\mathrm{~m}, 2 \mathrm{H}), 6.63$ (ddd, $J=8.1,2.3,1.1 \mathrm{~Hz}$, 1H), $5.39-5.19$ (m, 2H), 4.95 (d, $J=8.8 \mathrm{~Hz}, 1 \mathrm{H}), 3.70$ (ddd, $J=10.3,8.7,7.4 \mathrm{~Hz}, 1 \mathrm{H}), 3.40$ - $3.28(\mathrm{~m}, 2 \mathrm{H}), 2.80(\mathrm{~s}, 3 \mathrm{H}) . ;{ }^{13} \mathrm{C} \mathrm{NMR}\left(126 \mathrm{MHz}, \mathrm{CDCl}_{3}\right) \delta 170.2,150.5,140.7,136.9$, $131.1(\mathrm{q}, 2 J \mathrm{C}-\mathrm{F}=33.6 \mathrm{~Hz}), 129.1,128.0,127.1(\mathrm{q}, 3 J \mathrm{C}-\mathrm{F}=4.2 \mathrm{~Hz}), 126.8,124.7,121.4(\mathrm{sep}$, $3 J \mathrm{C}-\mathrm{F}=4.0 \mathrm{~Hz}), 122.0(\mathrm{q}, 1 J \mathrm{C}-\mathrm{F}=272.5 \mathrm{~Hz}), 122.0,120.9,115.3,110.7,70.3,64.3,56.7$, 53.5, 44.8; IR (film) $v_{\max } 2961,2359,1738,1591,1475,1394,1353,1278,1173,1133,1013$, 894, 843, 799, $765 \mathrm{~cm}^{-1}$. HRMS (ESI) calcd for $\mathrm{C}_{27} \mathrm{H}_{22} \mathrm{BrF}_{6} \mathrm{~N}_{2} \mathrm{O}_{4}{ }^{+}[\mathrm{M}+\mathrm{H}]^{+}$631.0662, found 631.0652. HPLC analysis: 96\% ee (CHIRALPAK IC, isopropanol/hexane $=3: 97,1.0 \mathrm{~mL} / \mathrm{min}$, $\mathrm{UV}: 254 \mathrm{~nm}$ ), $t_{\mathrm{R}}=4.359 \min$ (minor), $5.038 \mathrm{~min}$ (major).
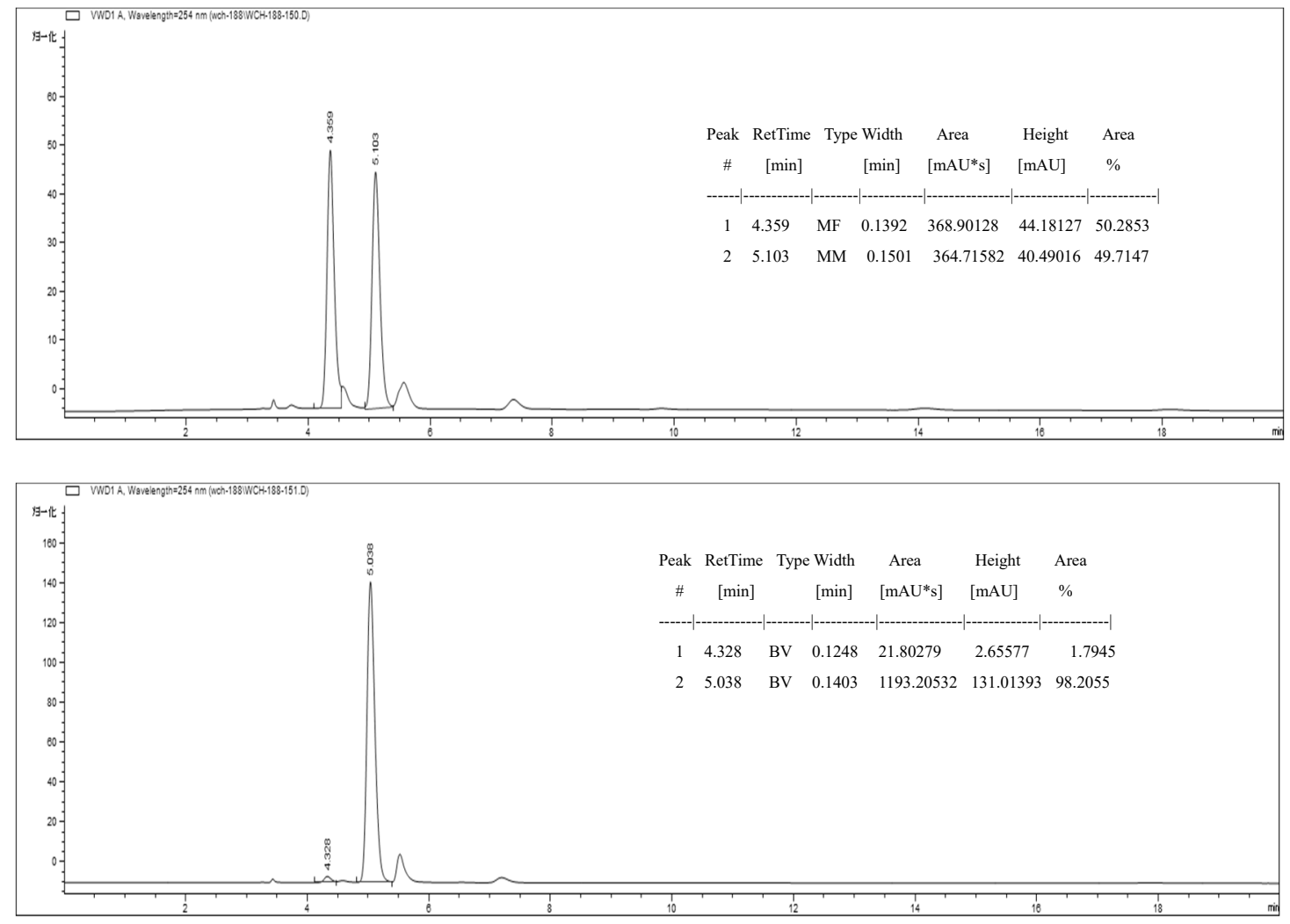
${ }^{1} \mathrm{H}$ and ${ }^{13} \mathrm{C}$ NMR Spectra

$\underbrace{-10}$<smiles>CC(=O)N1C=C(C(=O)OCc2cc(C(F)(F)F)cc(C(F)(F)F)c2)[C@H](c2ccccc2)N1c1ccccc1</smiles>

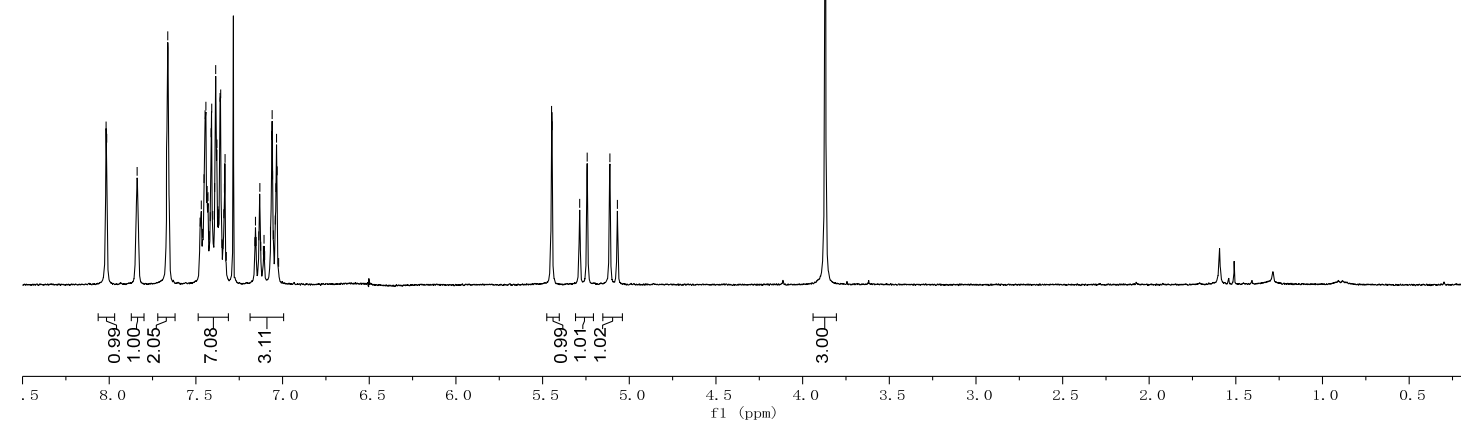

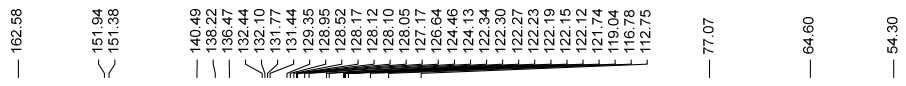

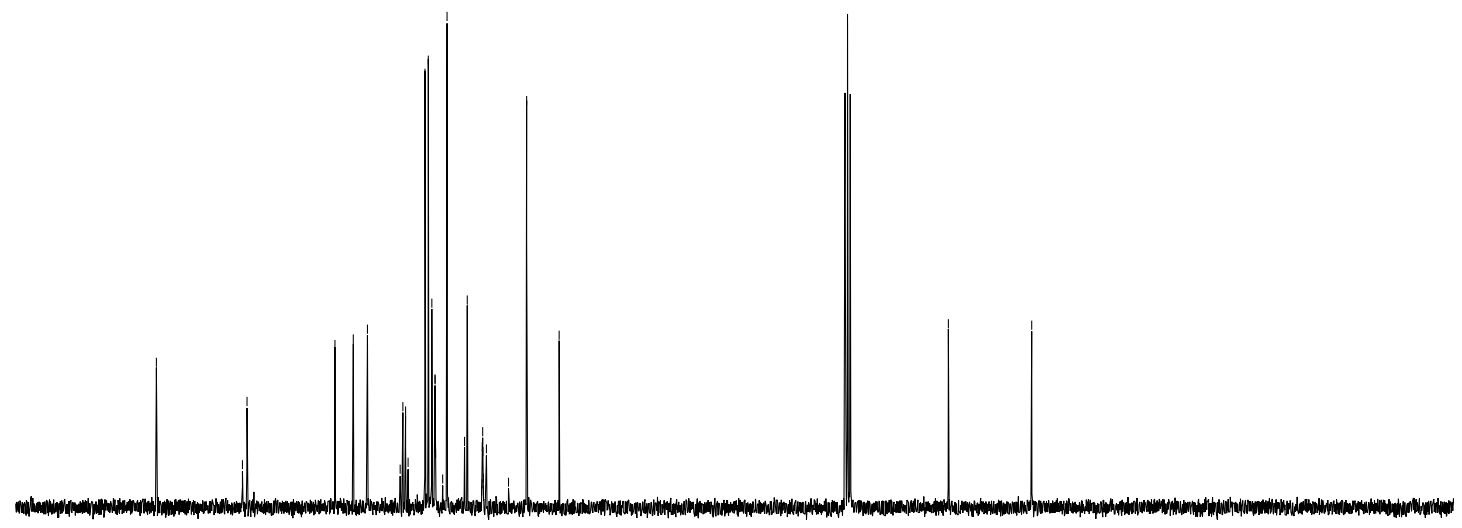

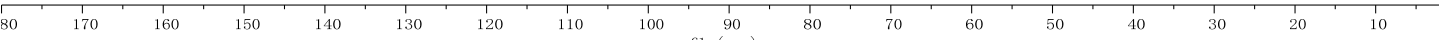




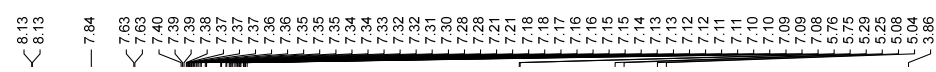<smiles>CC(=O)N1C=C(C(=O)OCc2cc(C(F)(F)F)cc(C(F)(F)F)c2)[C@H](c2ccccc2F)N1c1ccccc1</smiles>

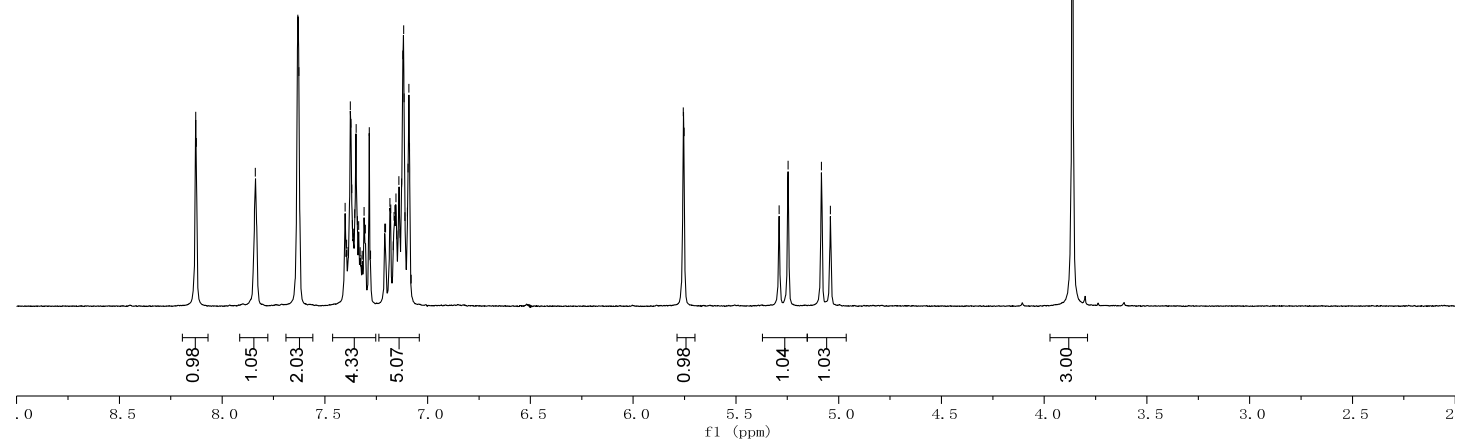

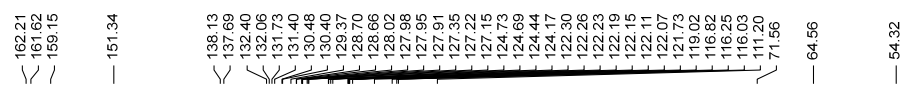

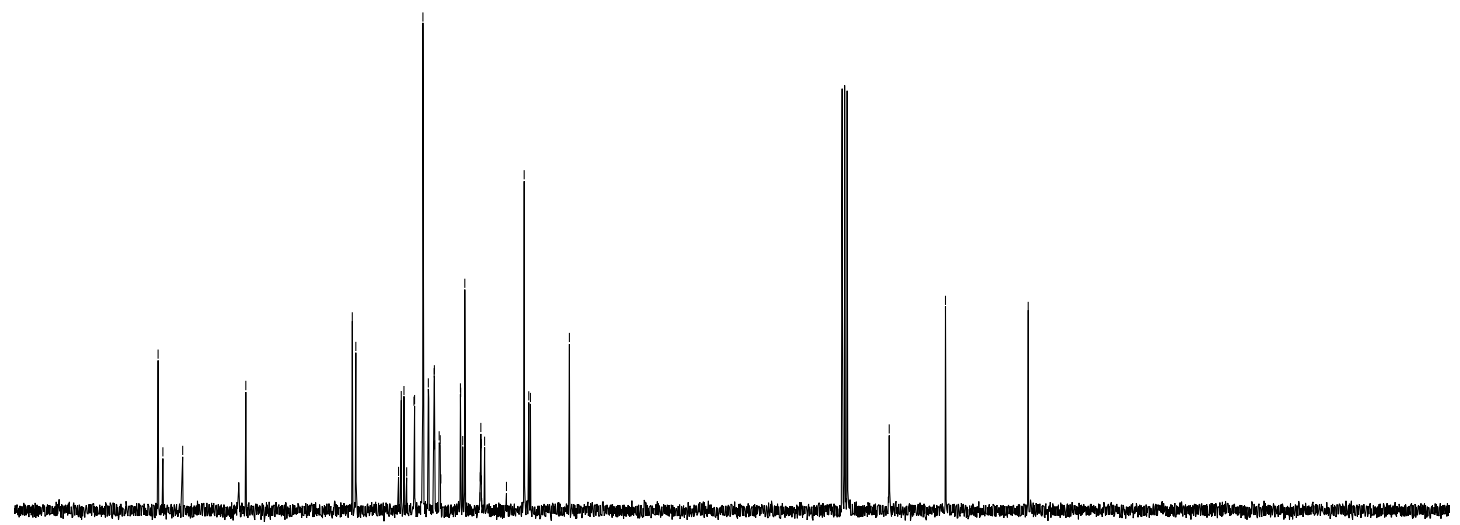

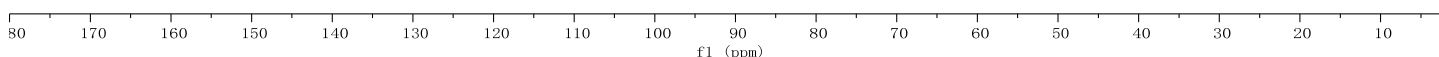


Y.

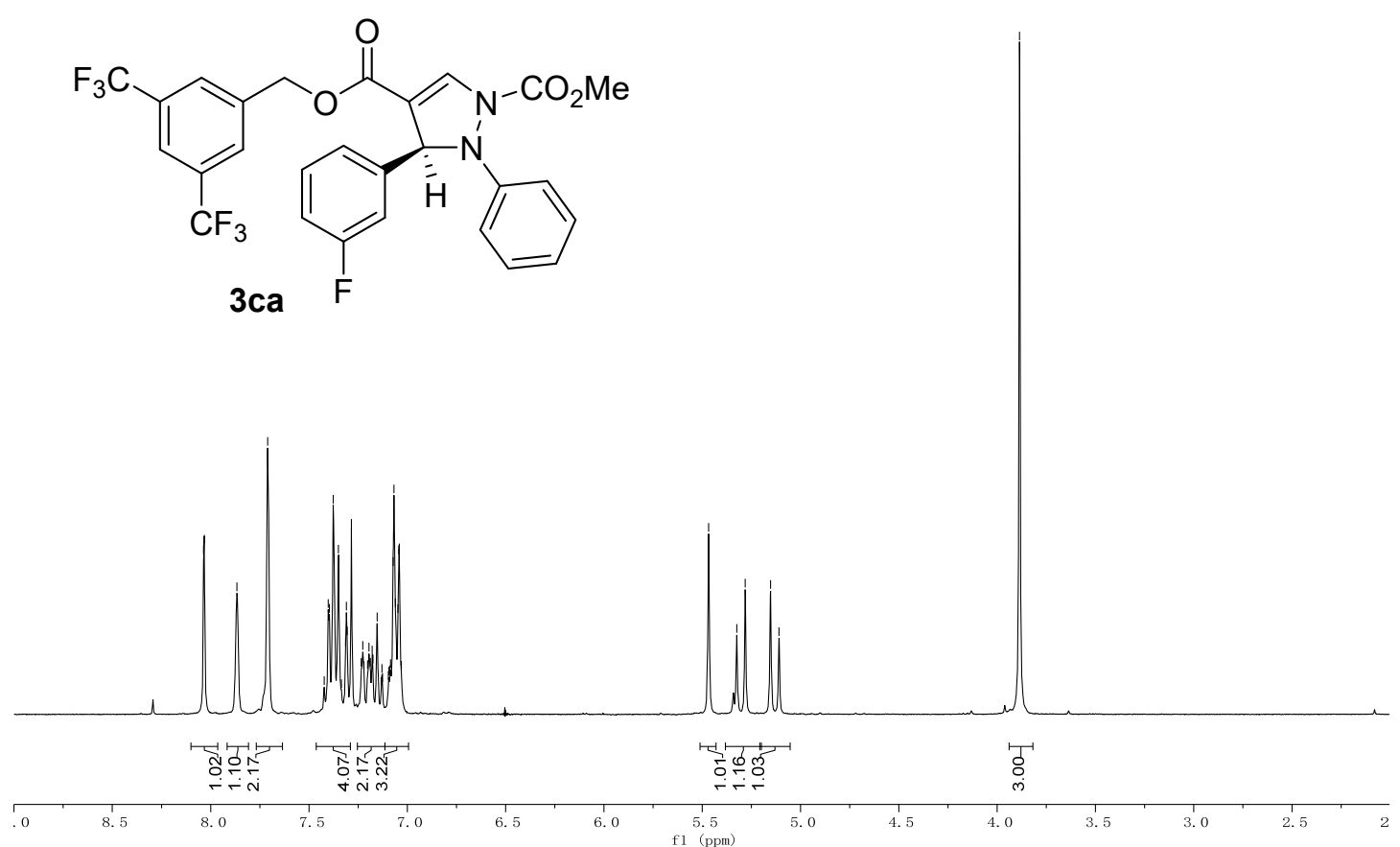

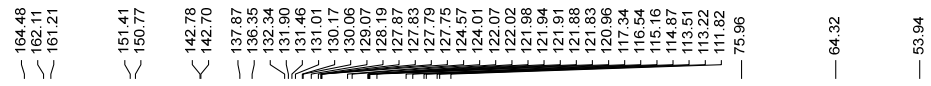

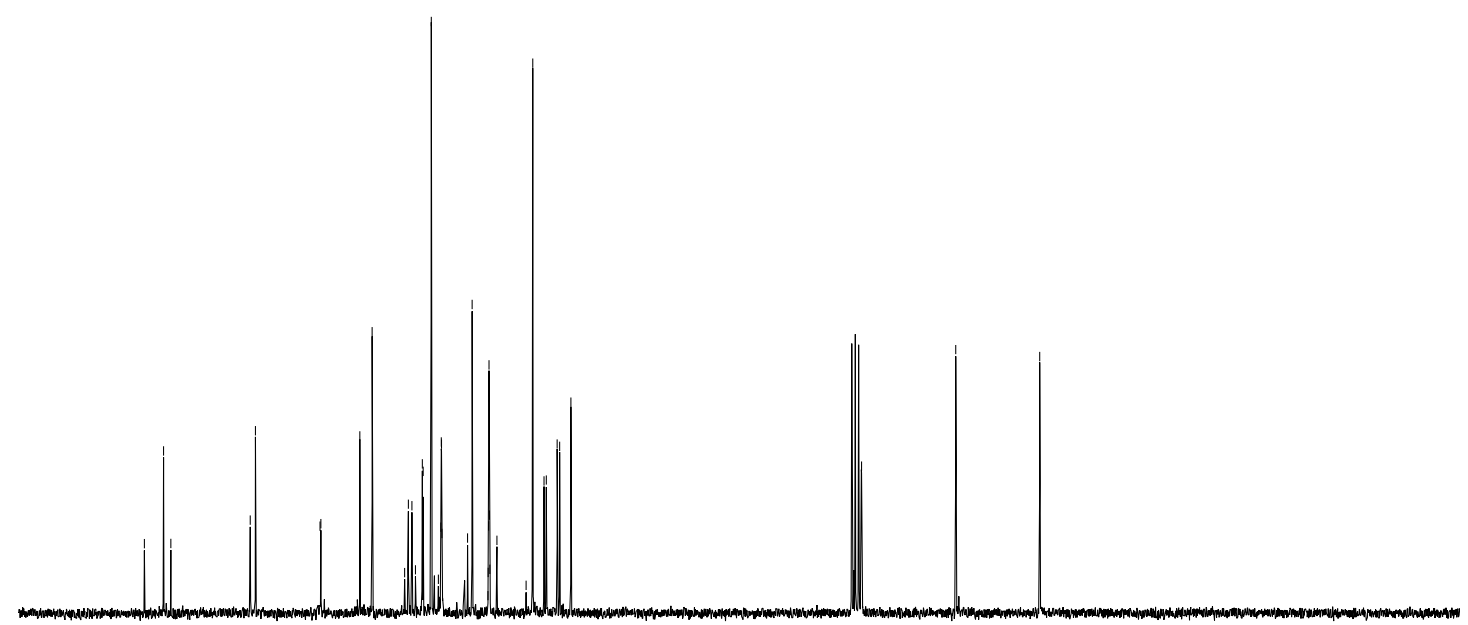

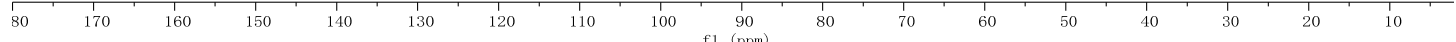




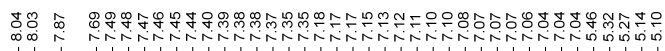<smiles>CC(=O)N1C=C(C(=O)OCc2cc(C(F)(F)F)cc(C(F)(F)F)c2)[C@H](c2ccc(C(F)(F)F)cc2)N1c1ccccc1</smiles>
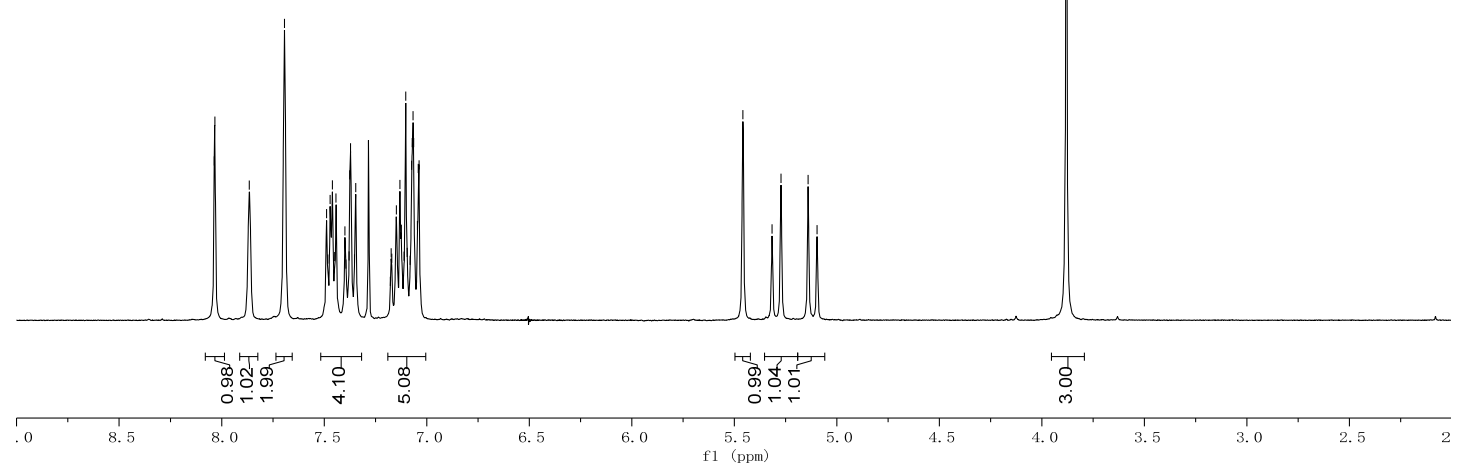

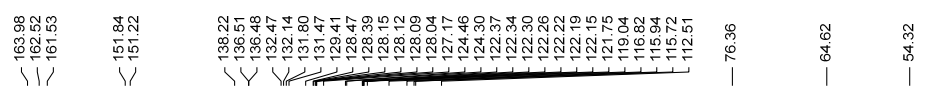

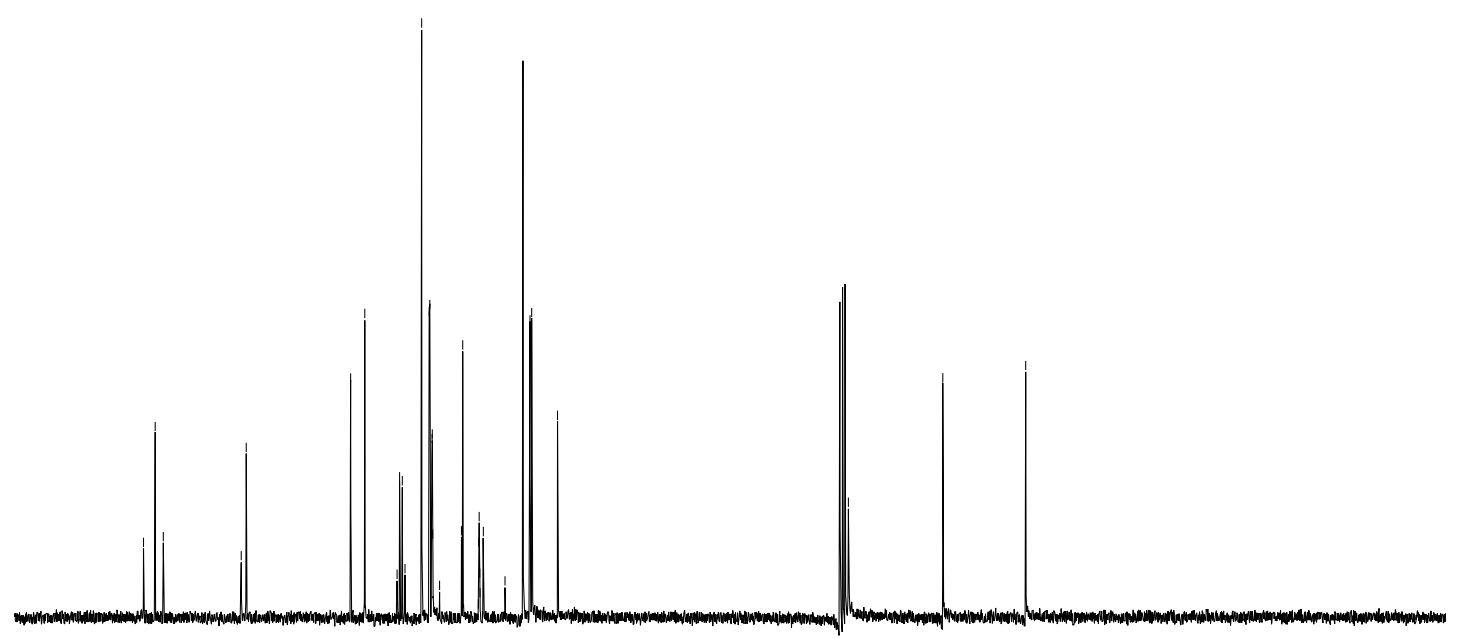

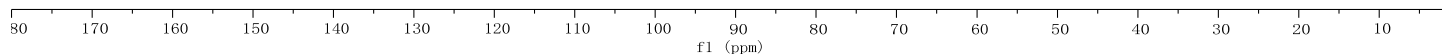




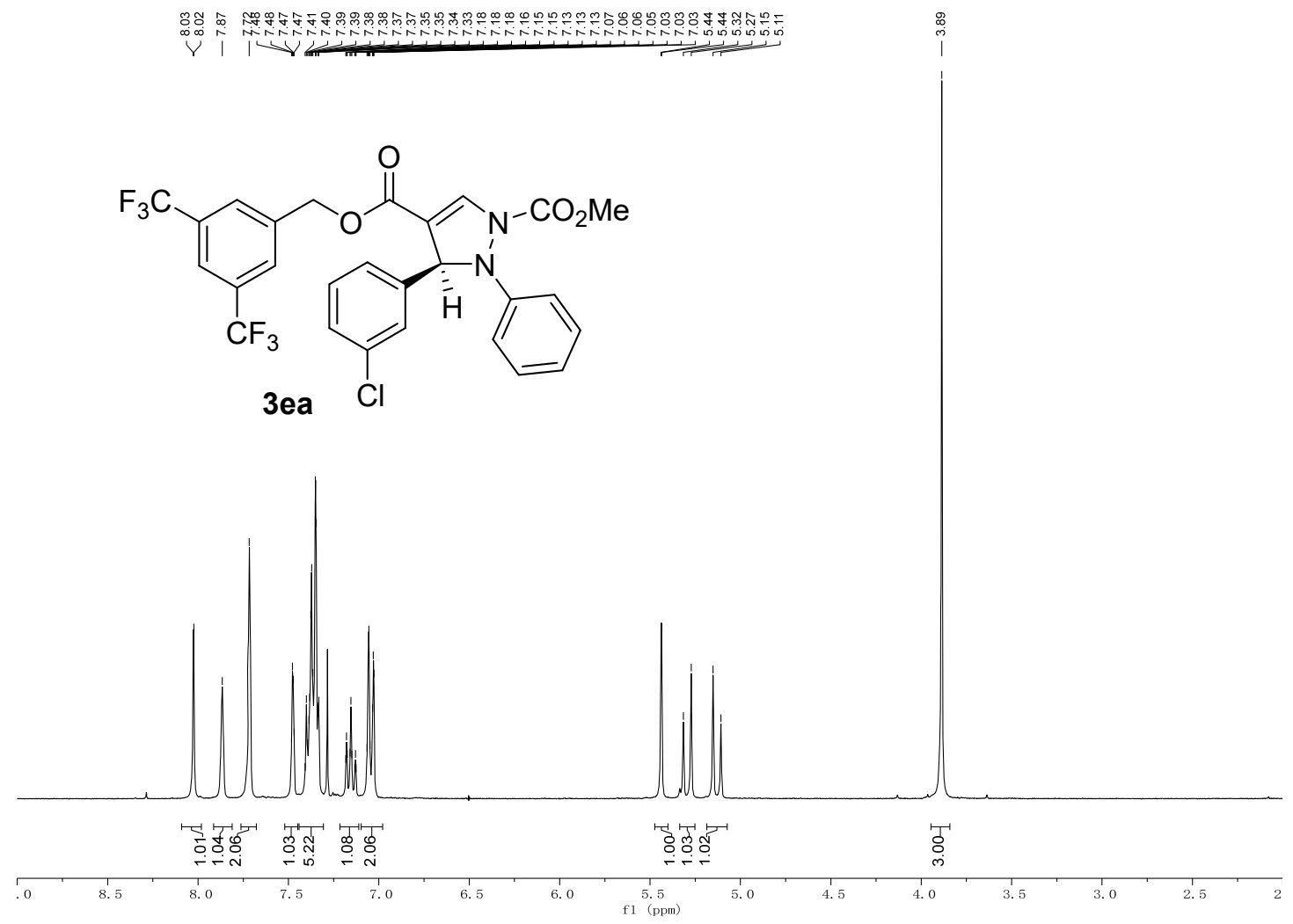

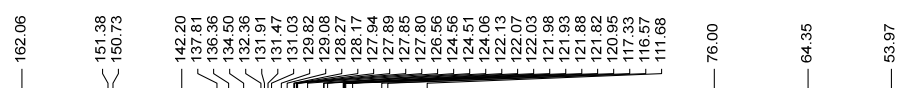

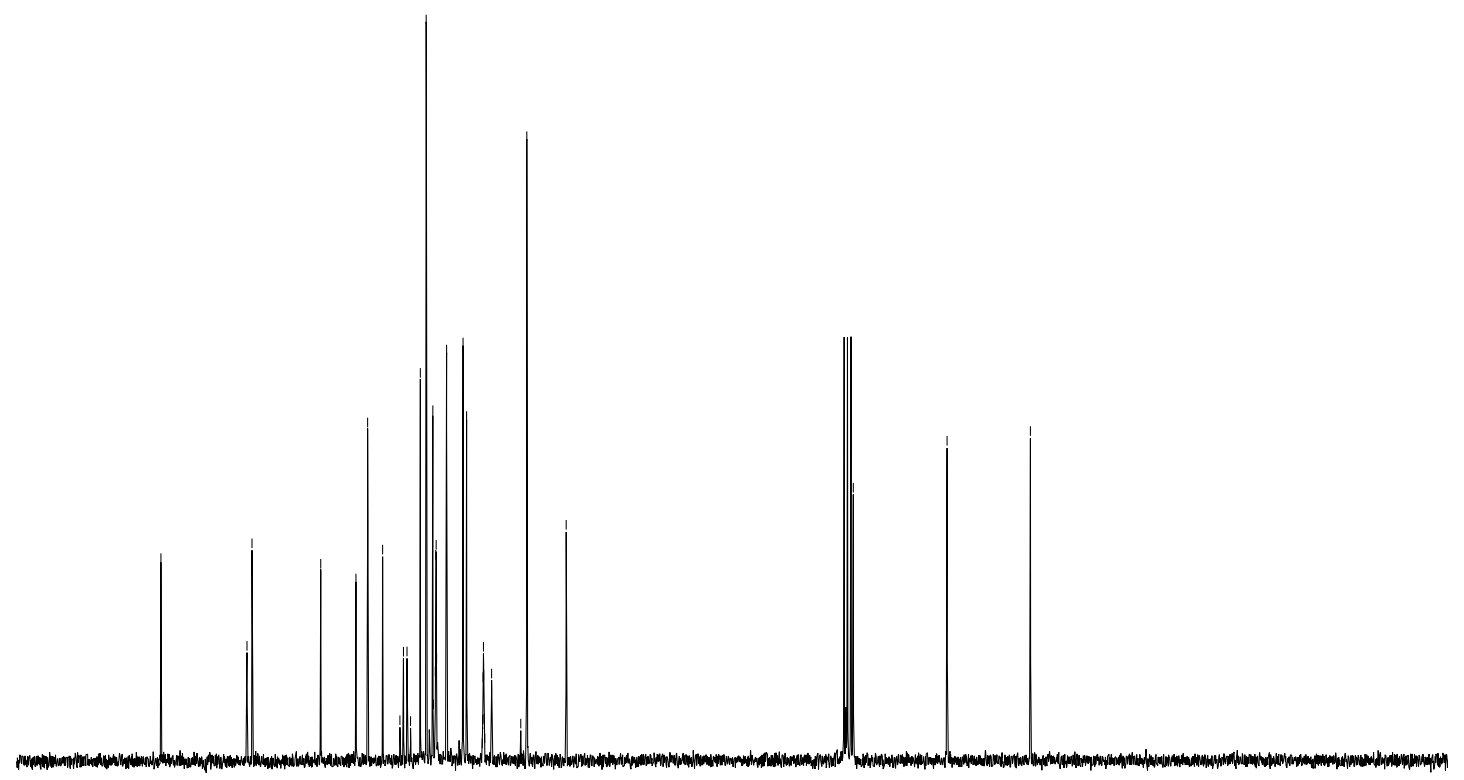

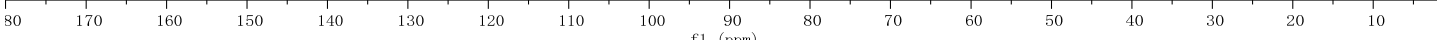




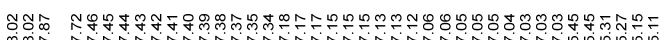<smiles>CC(=O)N1C=C(C(=O)OCc2cc(C(F)(F)F)cc(C(F)(F)F)c2)[C@H](c2ccc(Cl)cc2)N1c1ccccc1</smiles>

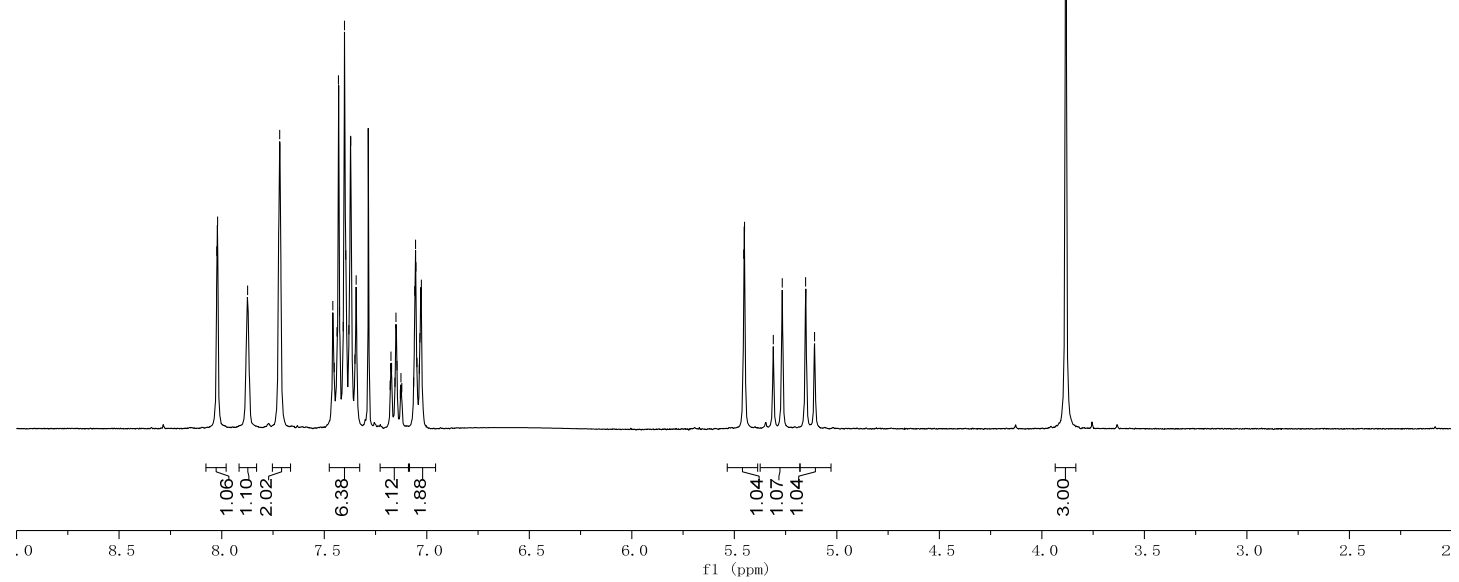

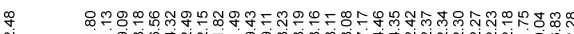

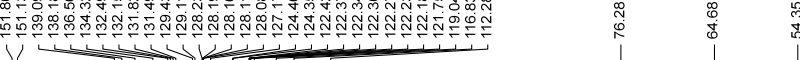
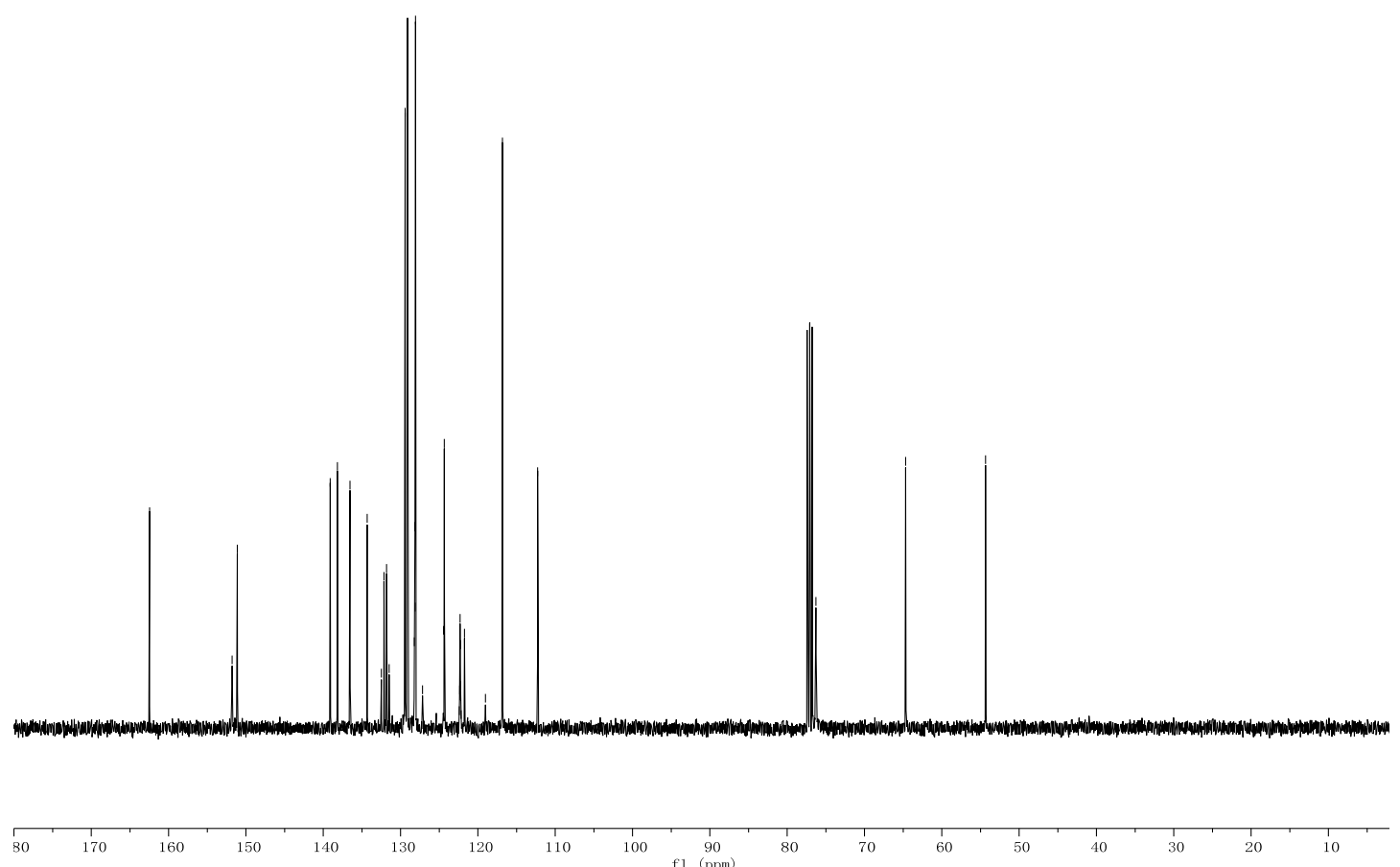
Y<smiles>CC(=O)N1C=C(C(=O)OCc2cc(C(F)(F)F)cc(C(F)(F)F)c2)C(c2ccccc2Br)N1c1ccccc1</smiles>

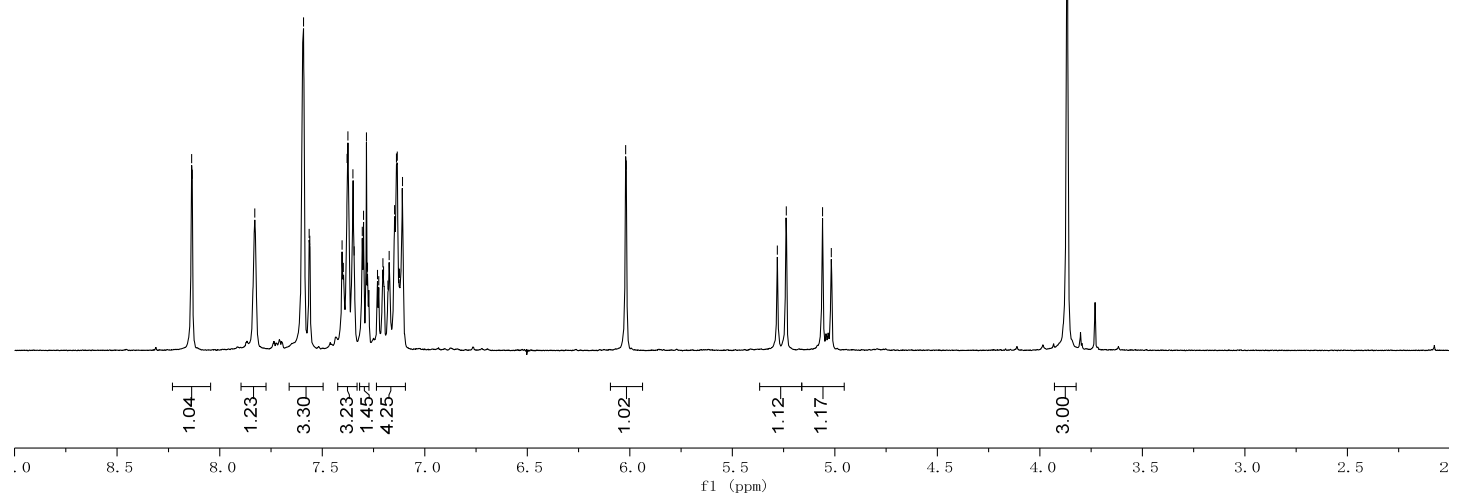

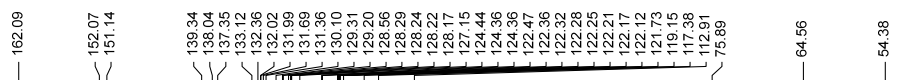
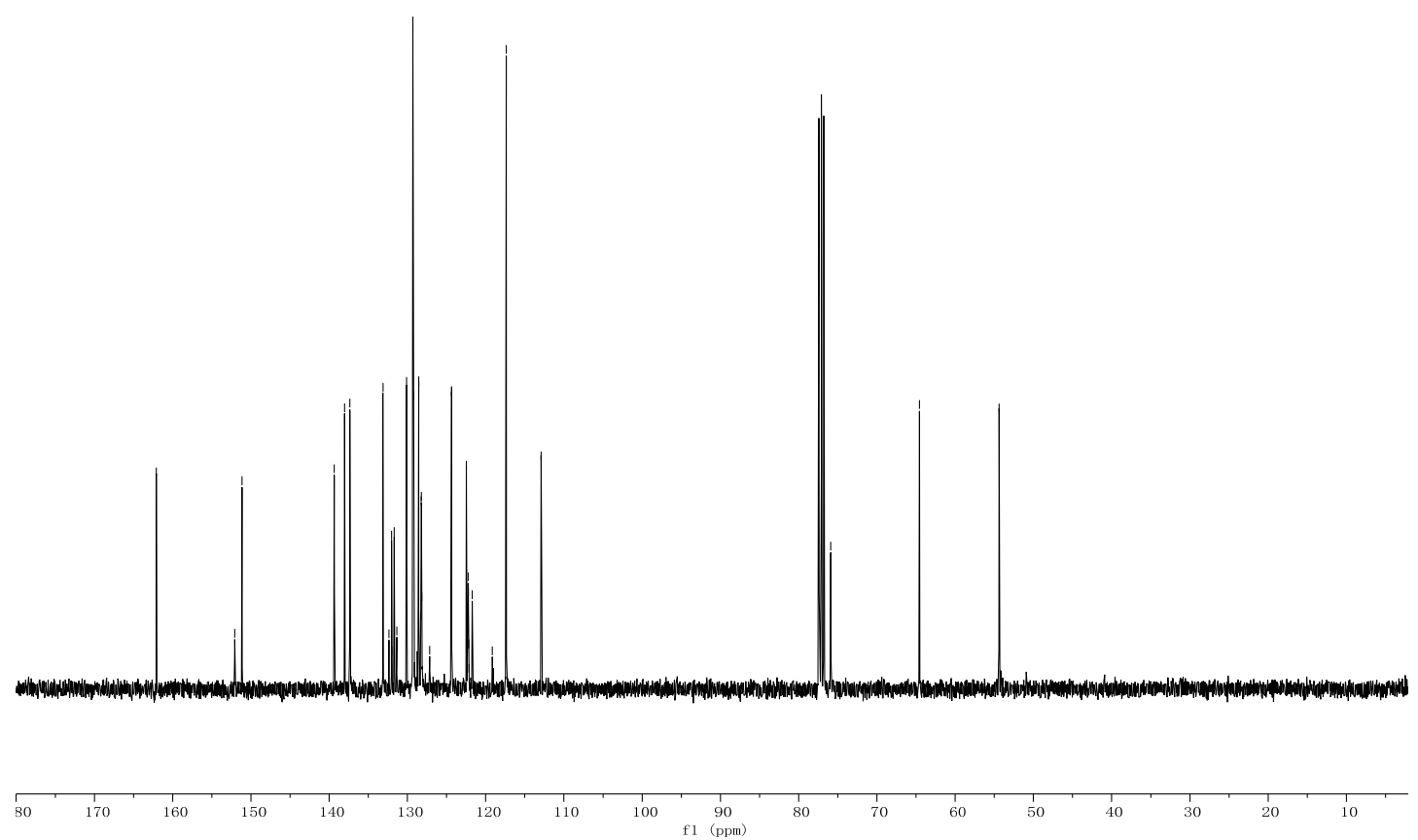
<smiles>CC(=O)N1C=C(C(=O)OCc2cc(C(F)(F)F)cc(C(F)(F)F)c2)C(c2ccc(Br)cc2)N1c1ccccc1</smiles>

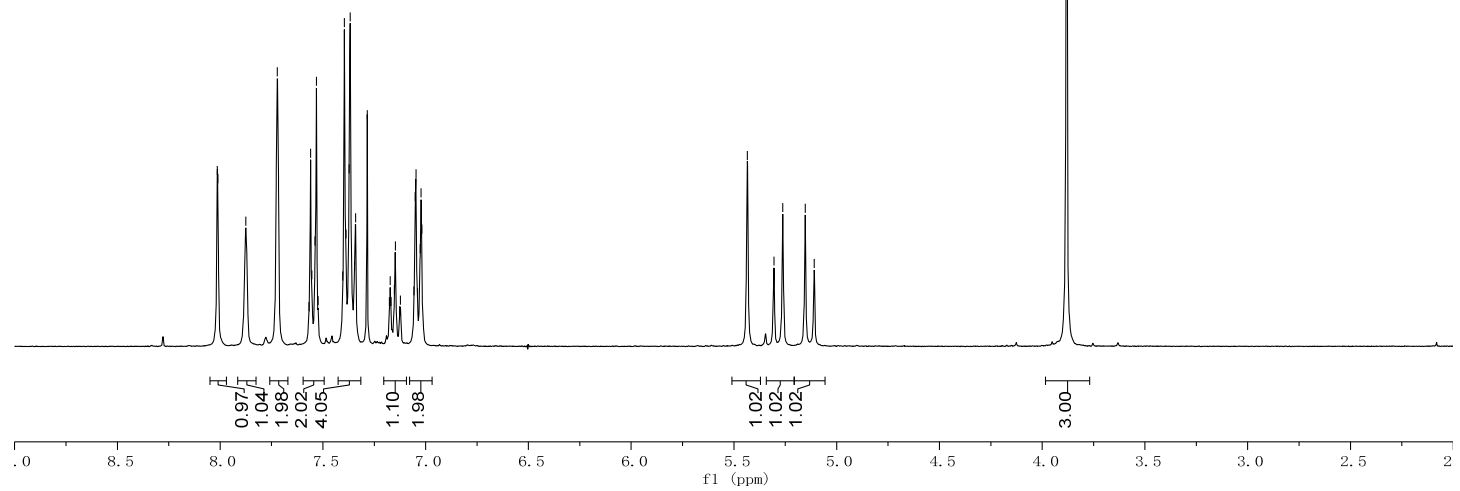

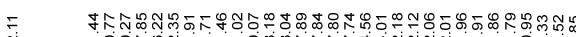
i

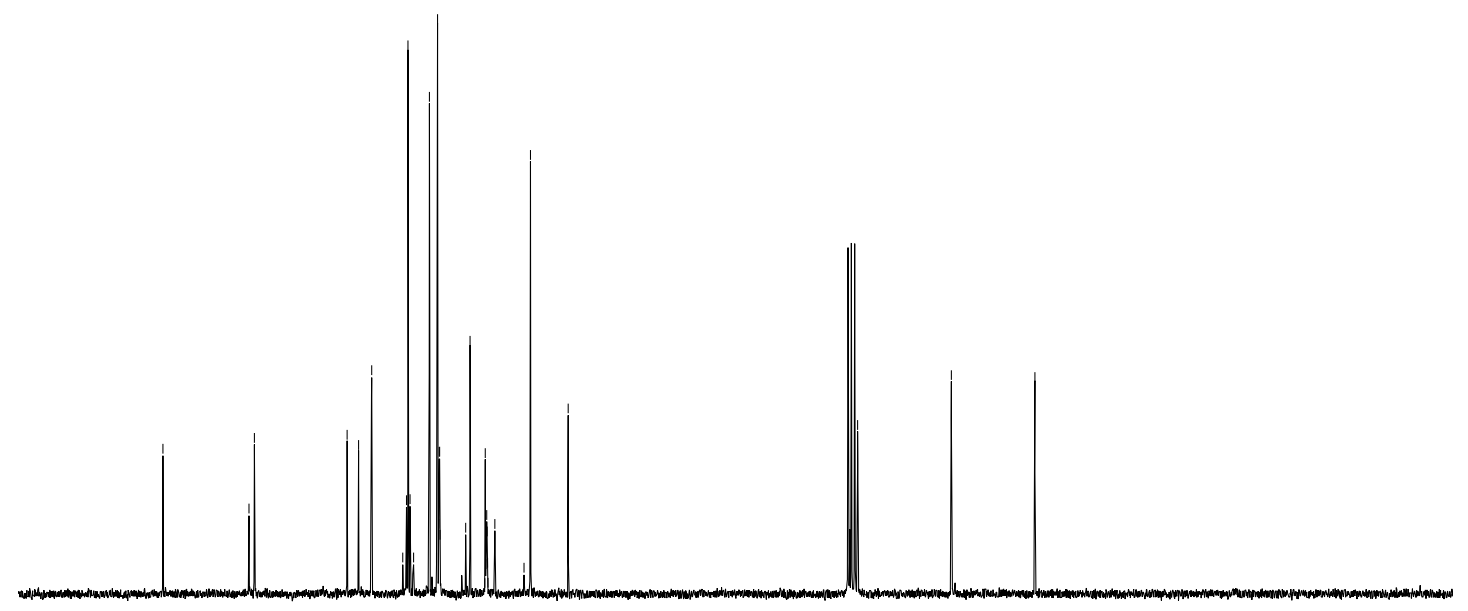

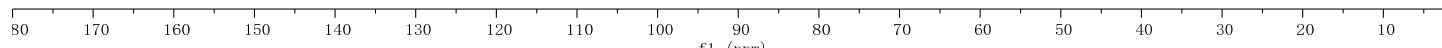




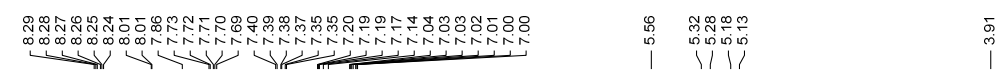<smiles>CC(=O)N1C=C(C(=O)OCc2cc(C(F)(F)F)cc(C(F)(F)F)c2)[C@H](c2ccc([N+](=O)[O-])cc2)N1c1ccccc1</smiles>
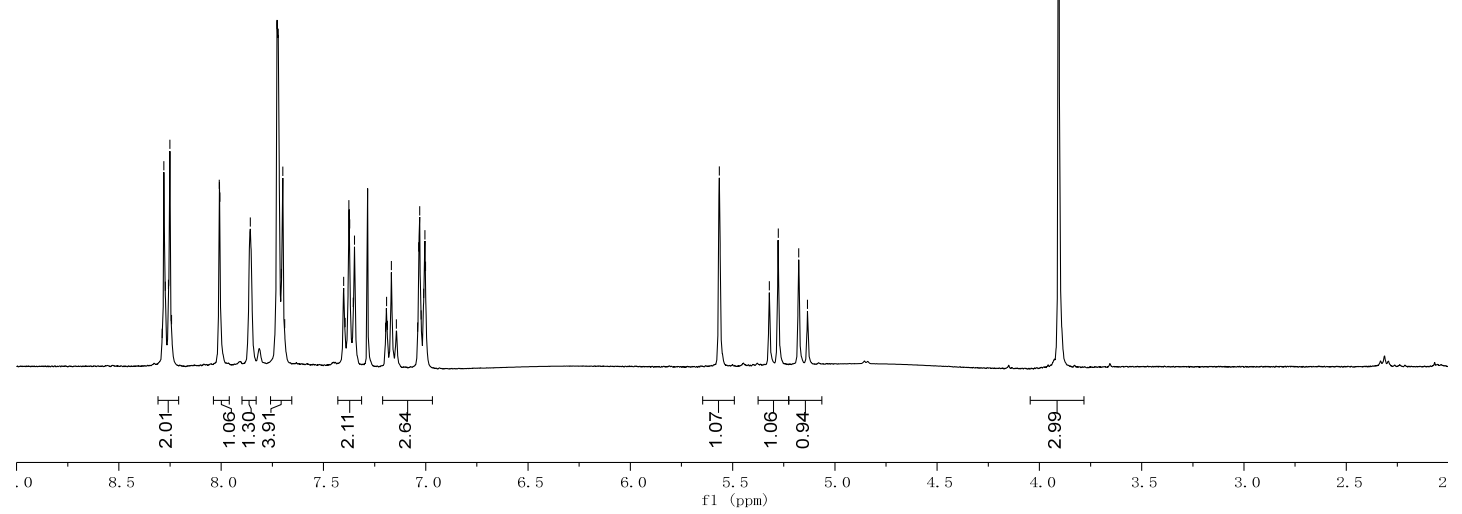

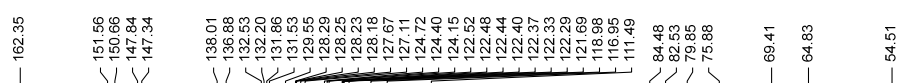
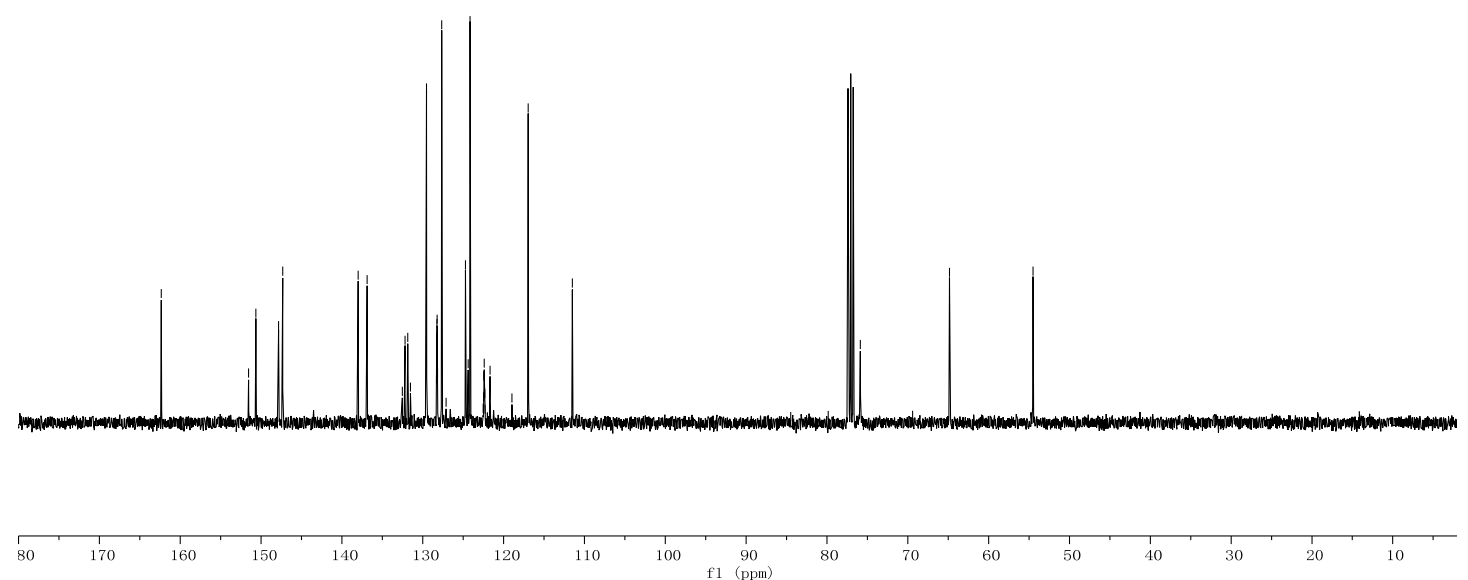


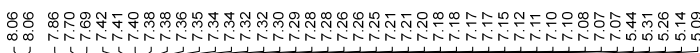<smiles>CC(=O)N1C=C(C(=O)OCc2cc(C(F)(F)F)cc(C(F)(F)F)c2)C(c2cccc(C)c2)[C@H]1c1ccccc1</smiles>
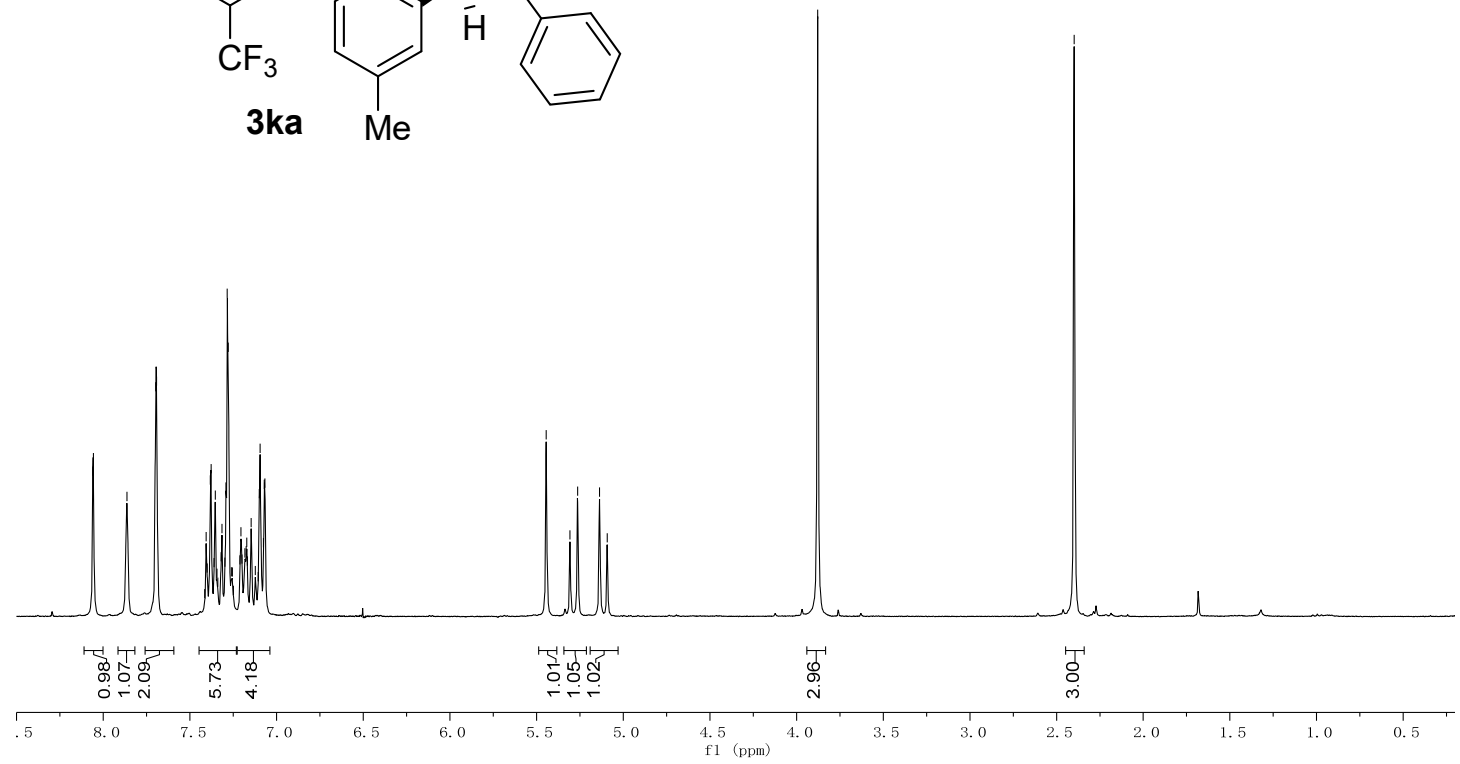

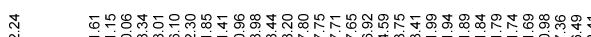

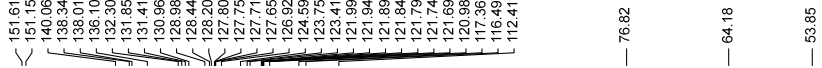

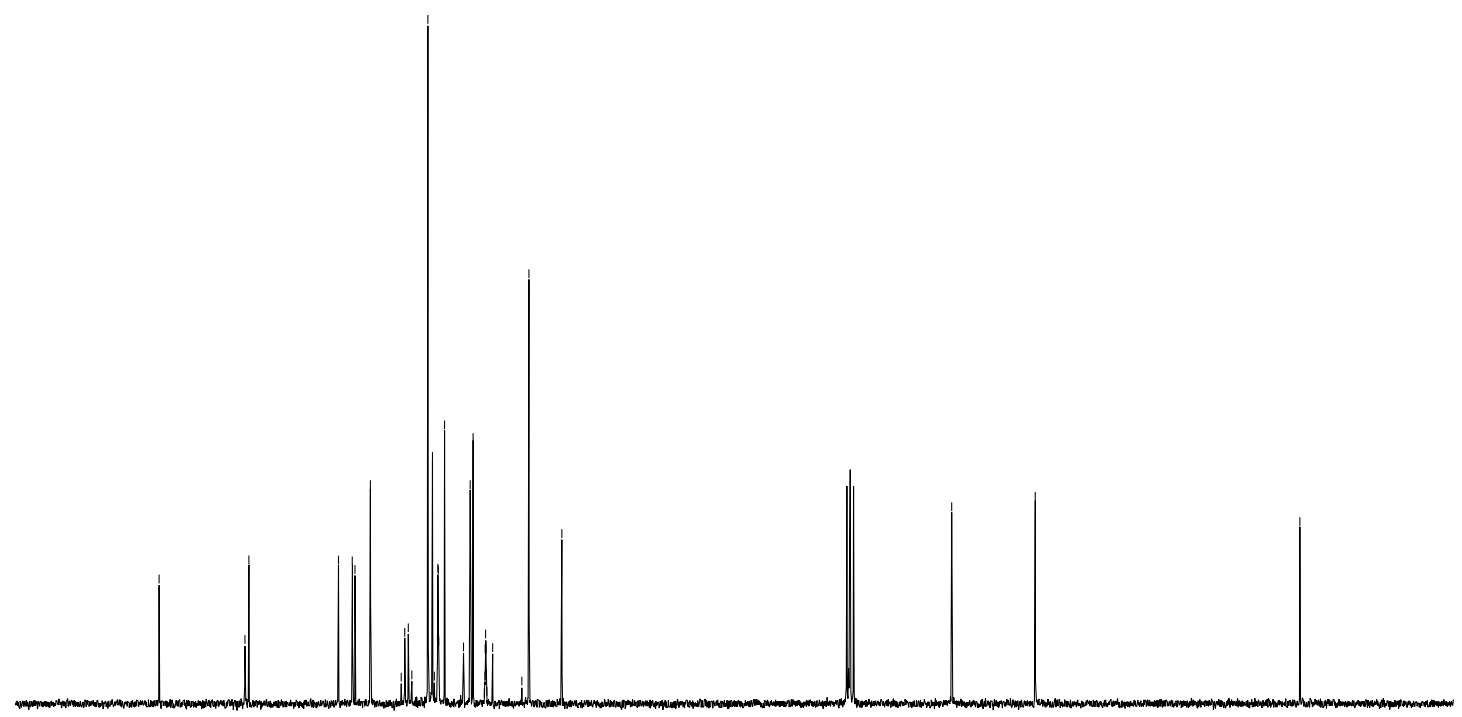

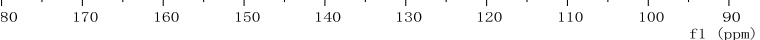


<smiles>CC(=O)N1C=C(C(=O)OCc2cc(C(F)(F)F)cc(C(F)(F)F)c2)[C@H](c2ccc(C)cc2)N1c1ccccc1</smiles>

3la
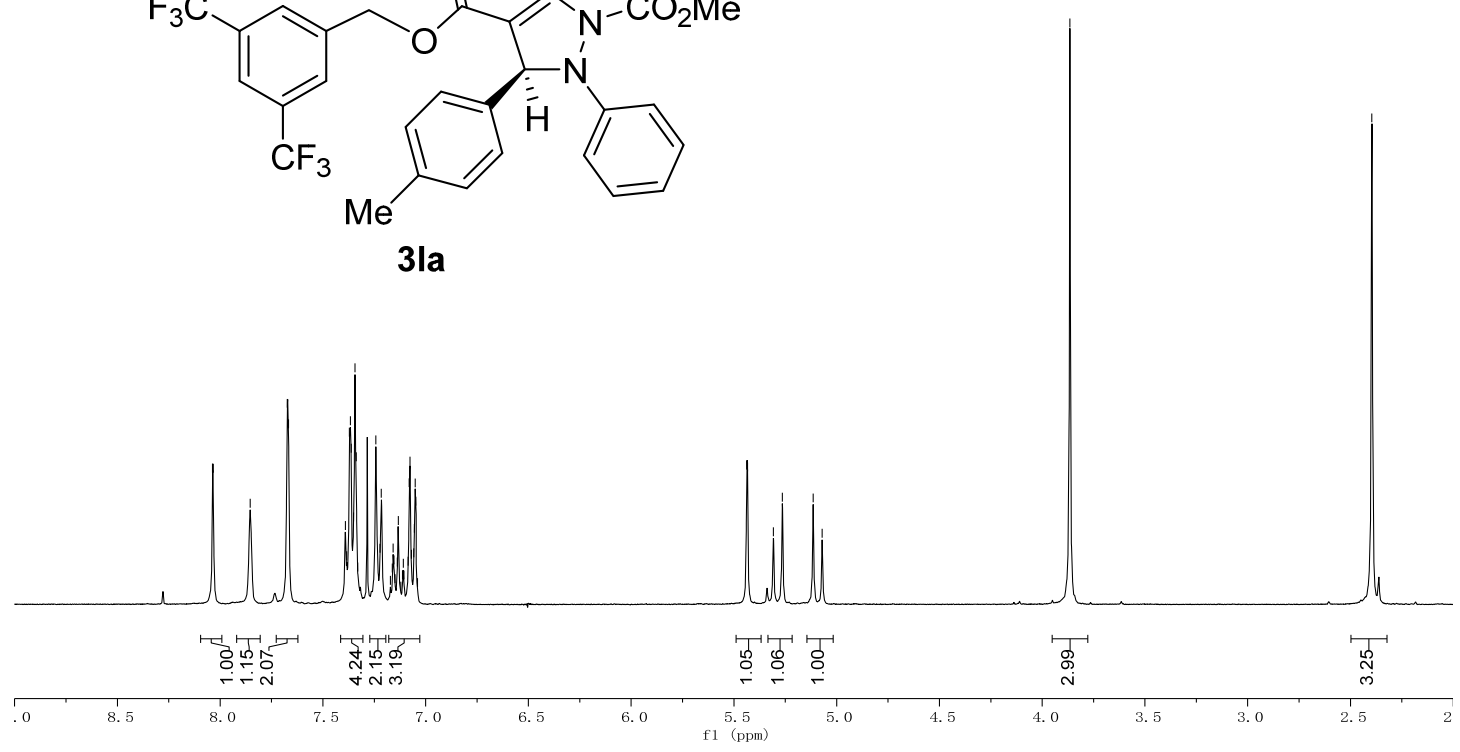

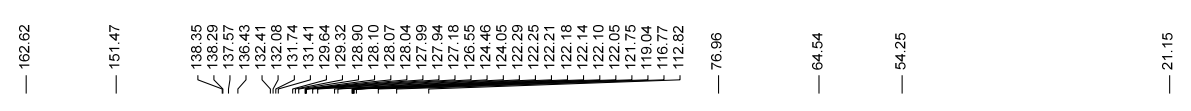

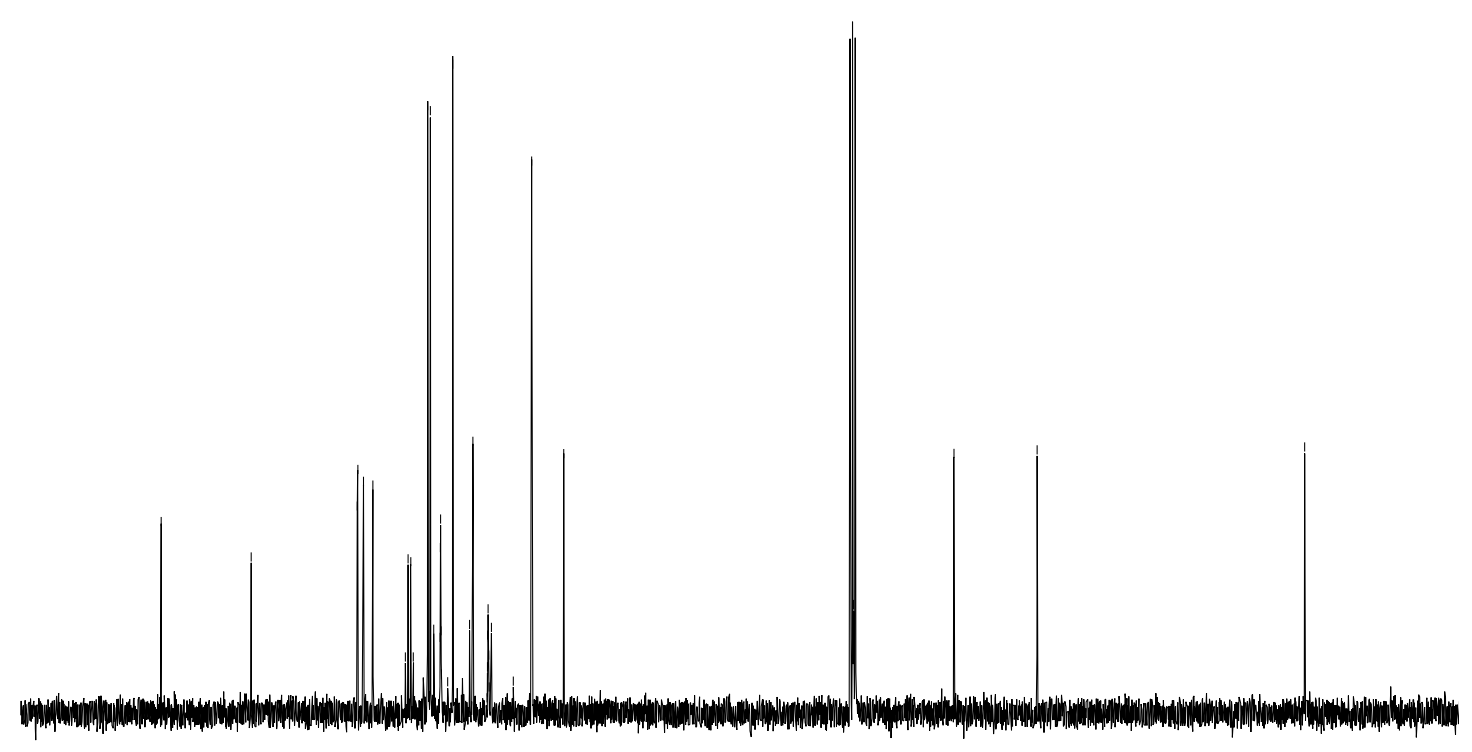

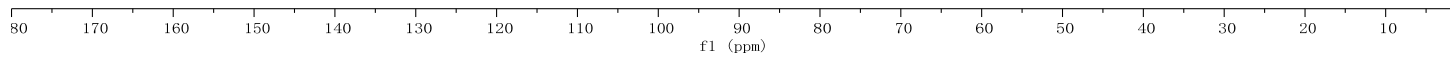


$1 \quad 1 \quad$

(ma
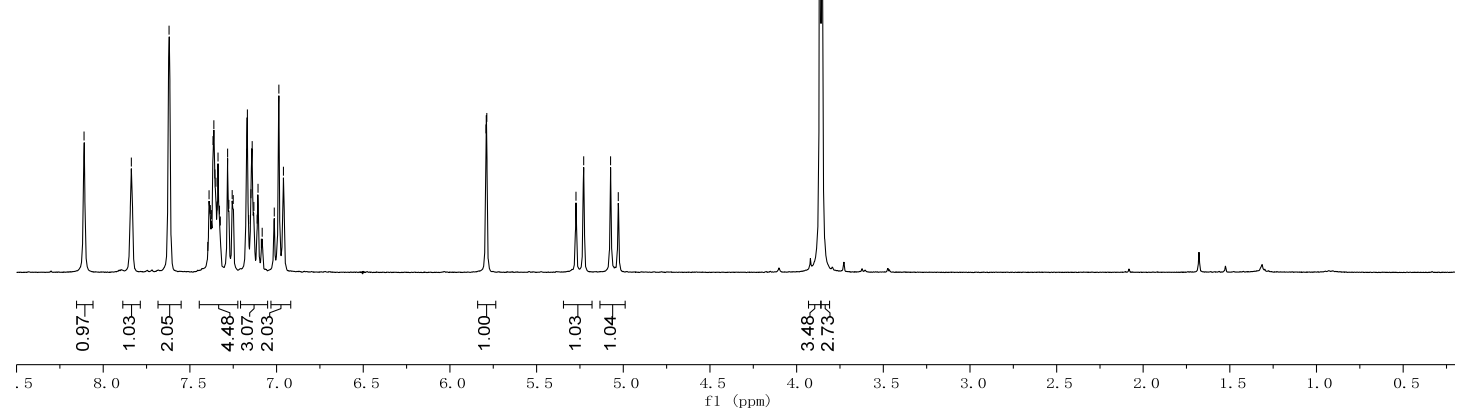

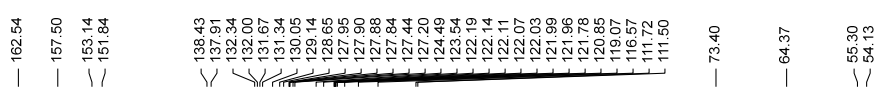

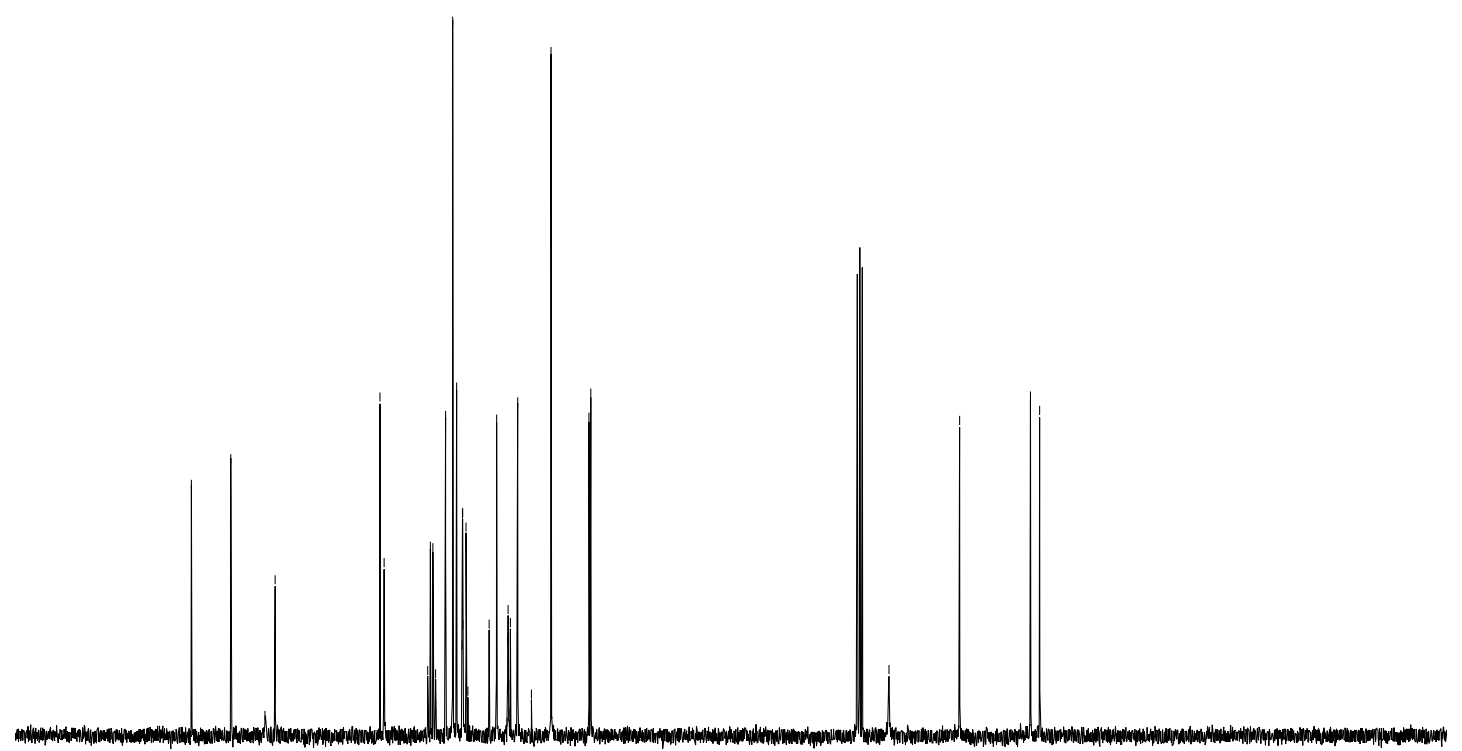

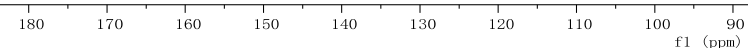




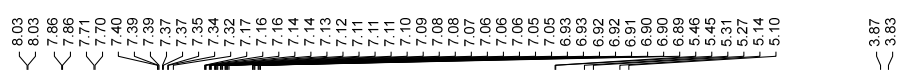<smiles>COc1cccc([C@H]2C(C(=O)OCc3cc([14CH3])cc(C(F)(F)F)c3)=CN(C(C)=O)N2c2ccccc2)c1</smiles>

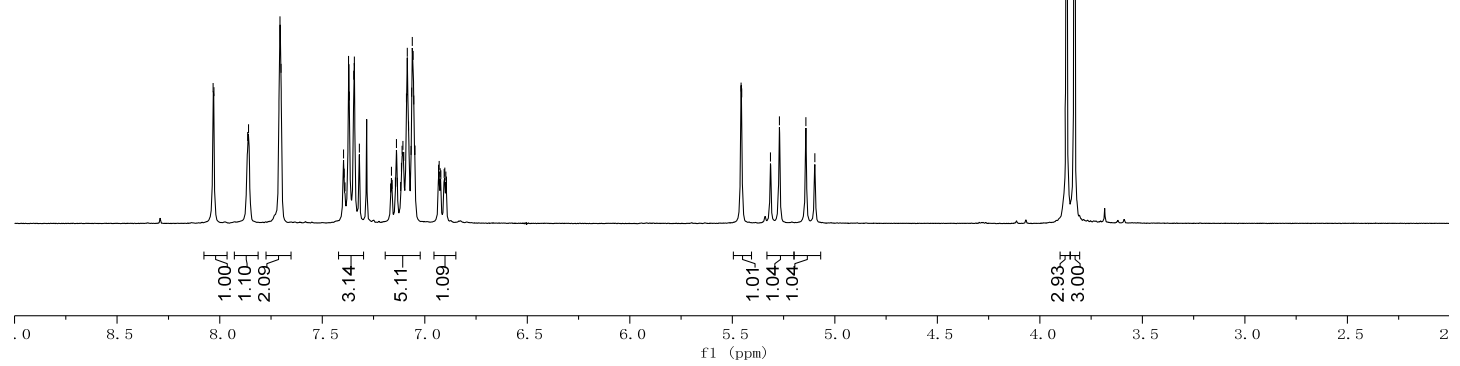

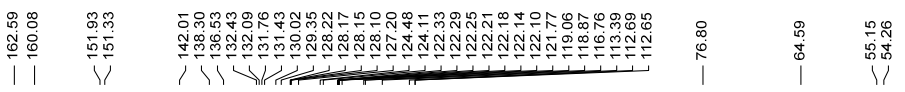

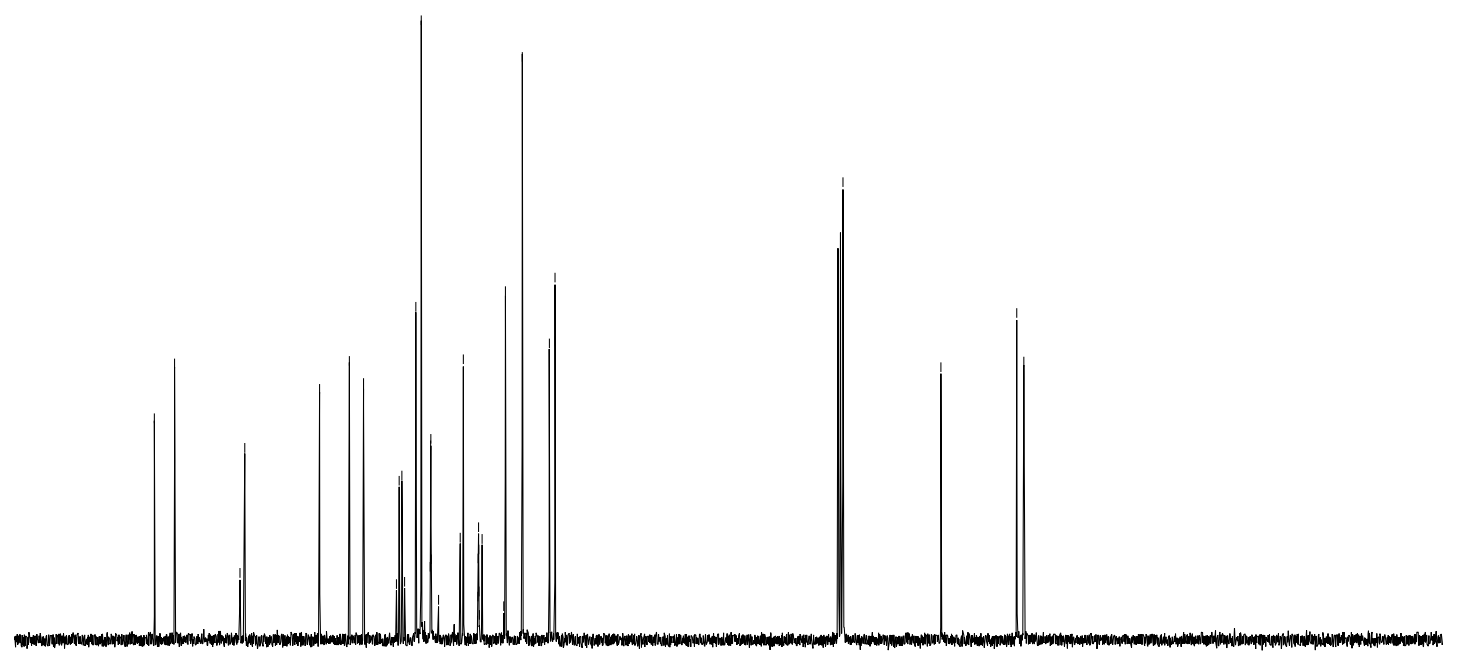

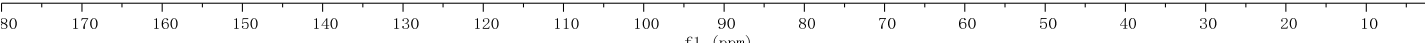


<smiles>COc1ccc([C@H]2C(C(=O)OCc3cc(C(F)(F)F)cc(C(F)(F)F)c3)=CN(C(C)=O)N2c2ccccc2)cc1</smiles>

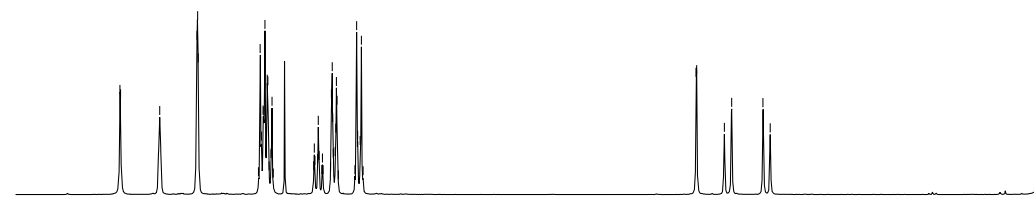

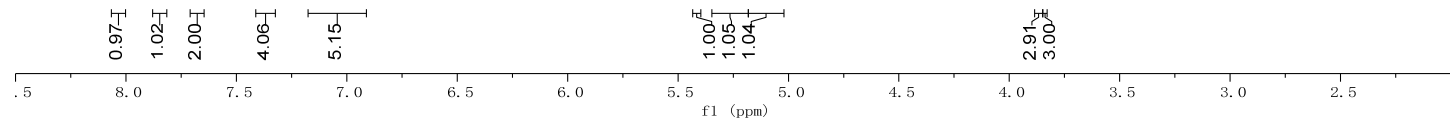
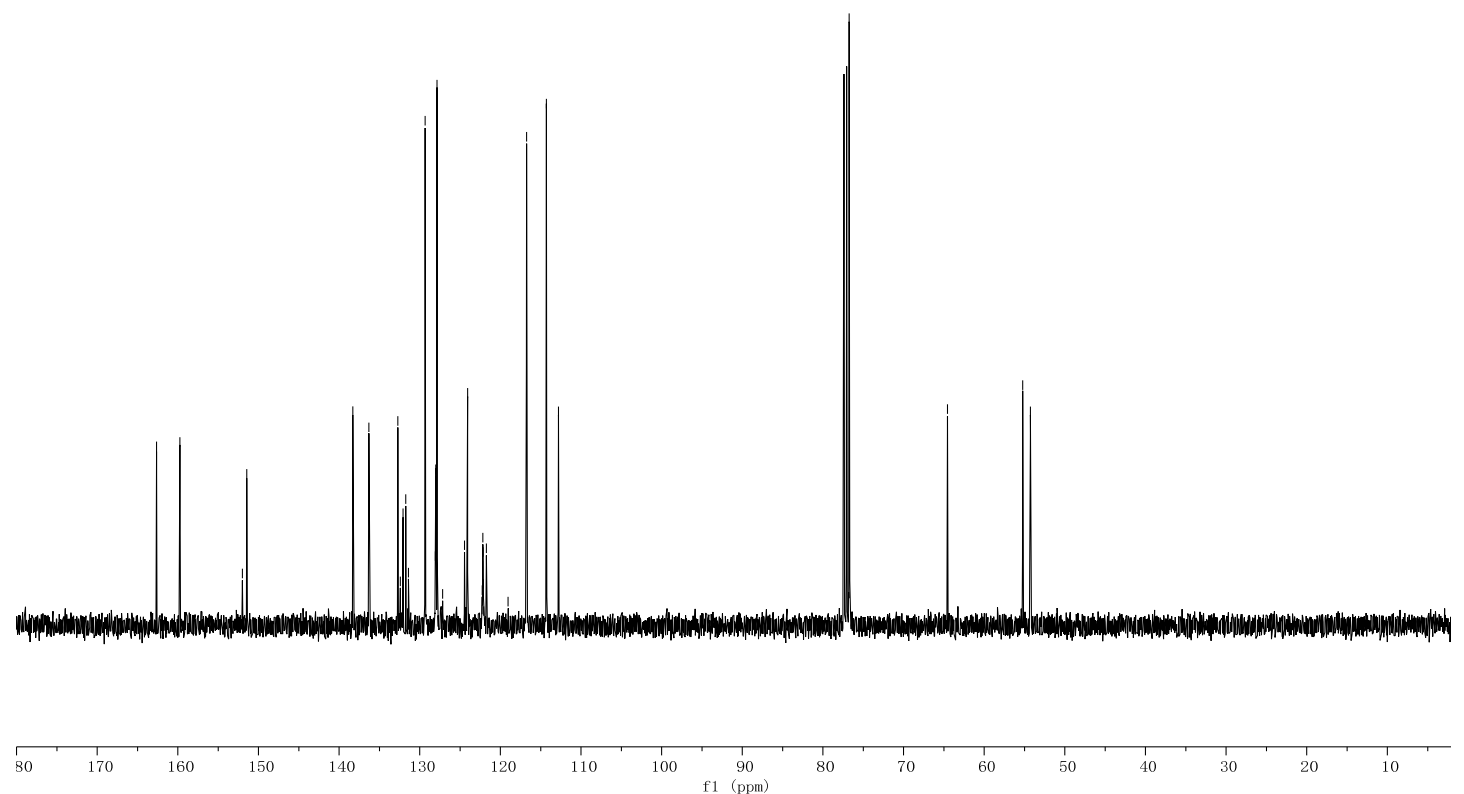


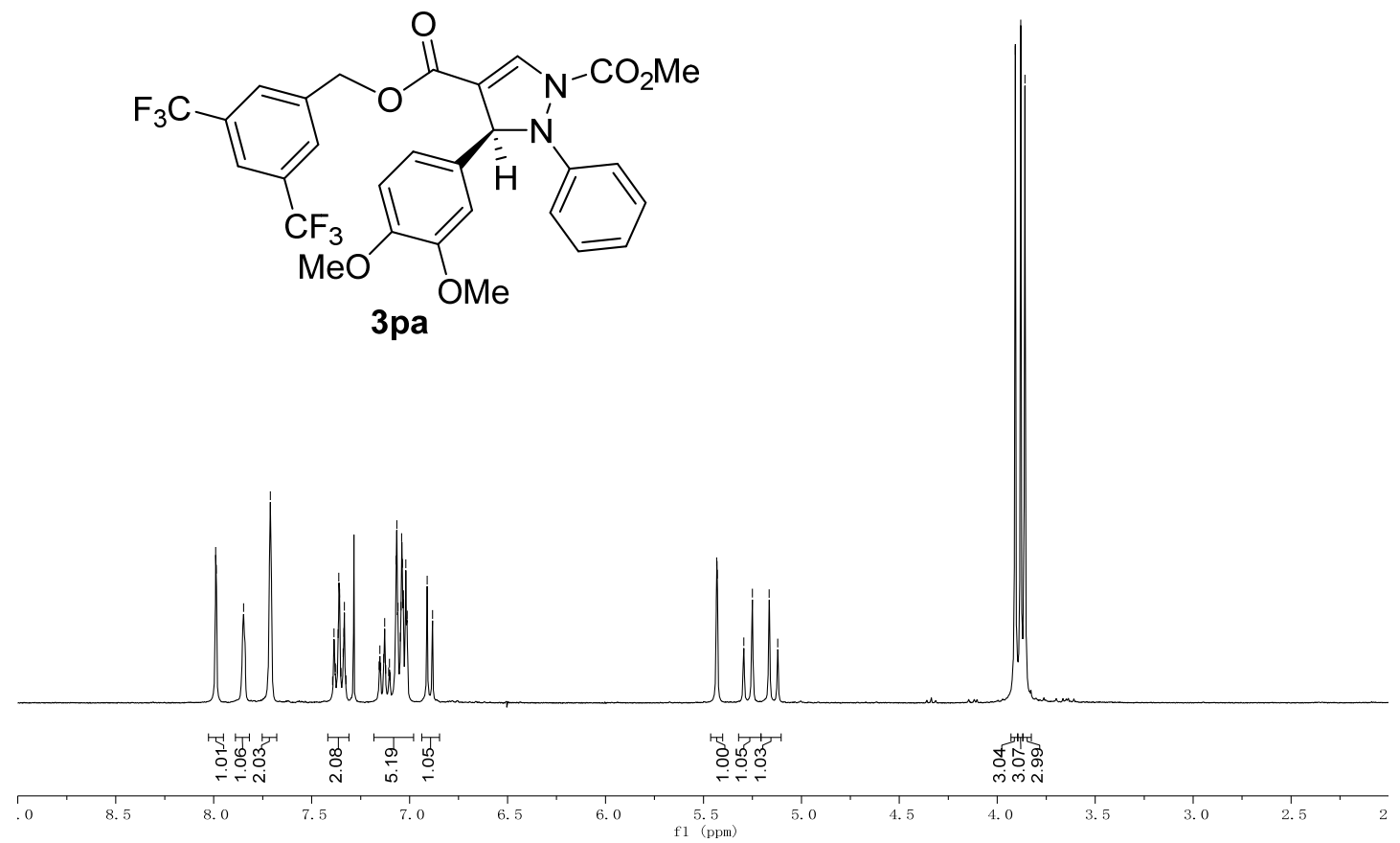
|

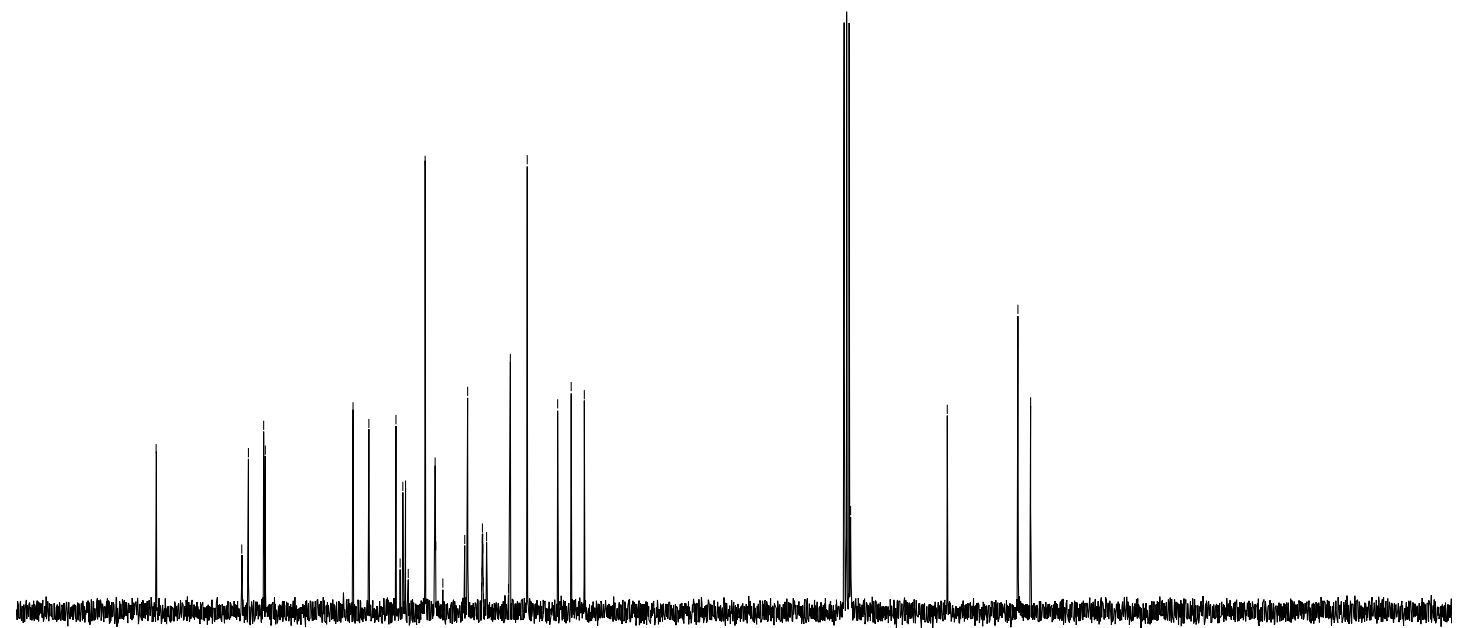


<smiles>CC(=O)N1C=C(C(=O)OCc2cc(C(F)(F)F)cc(C(F)(F)F)c2)[C@H](c2ccc3ccccc3c2)N1c1ccccc1</smiles>
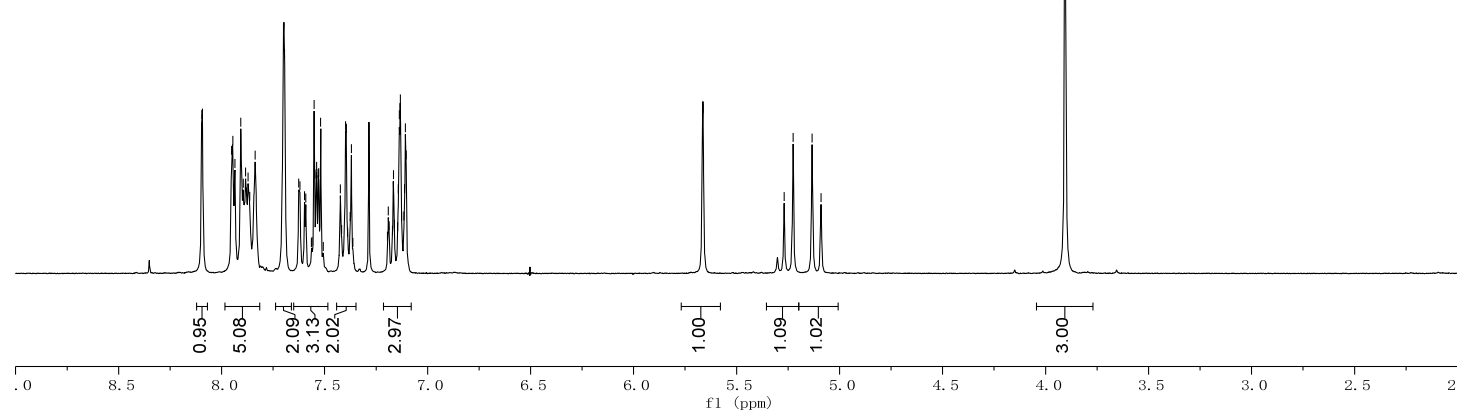

|

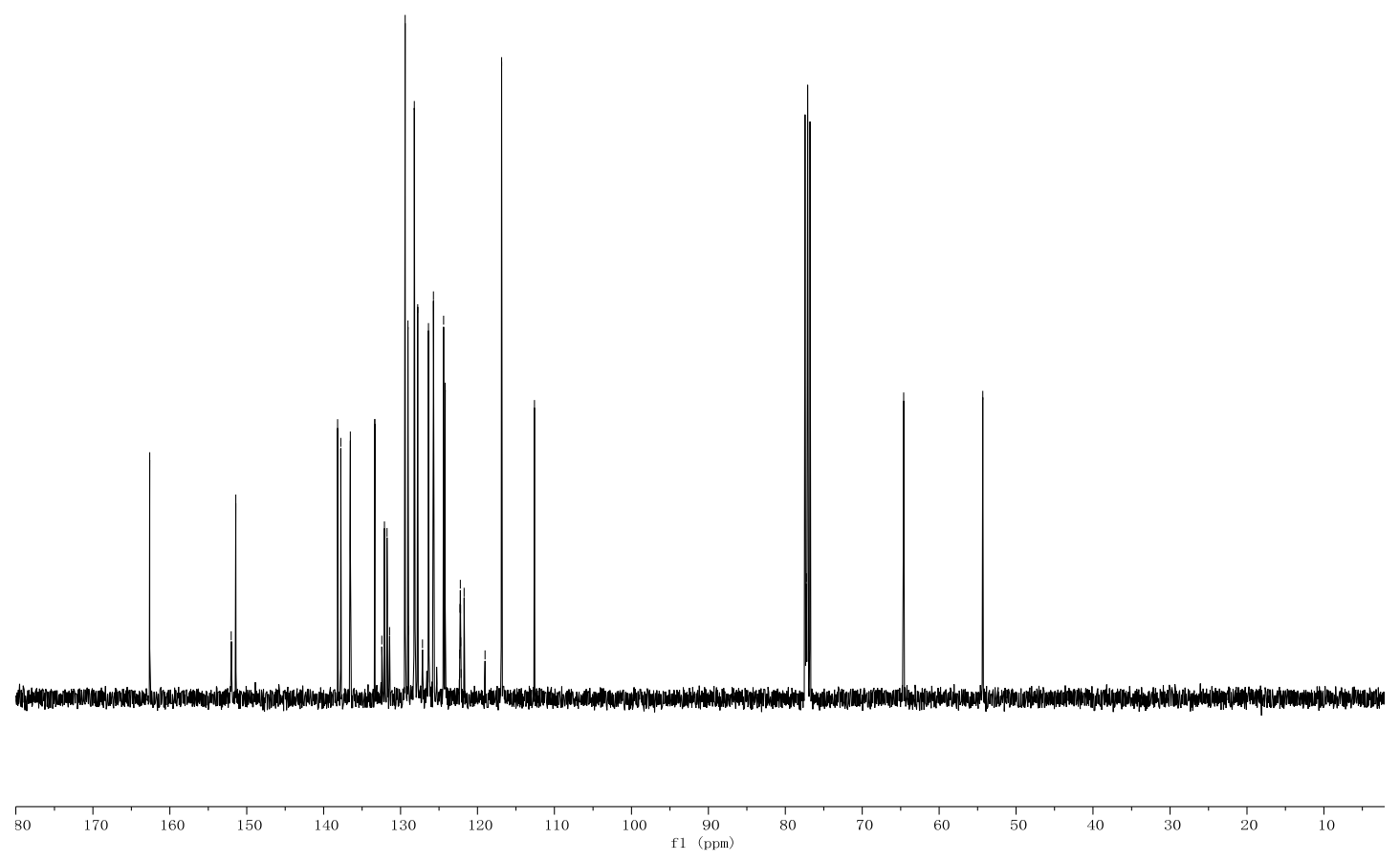




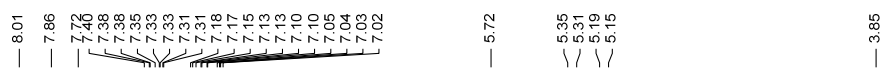<smiles>CC(=O)N1C=C(C(=O)OCc2cc(C(F)(F)F)cc(C(F)(F)F)c2)[C@H](c2cccs2)N1c1ccccc1</smiles>

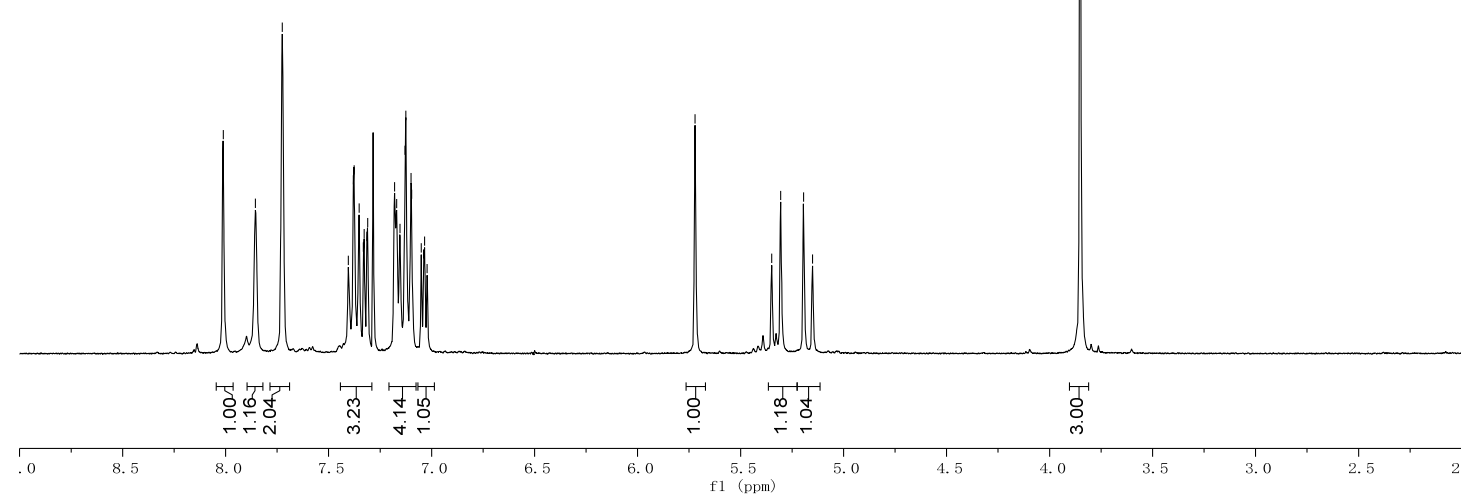

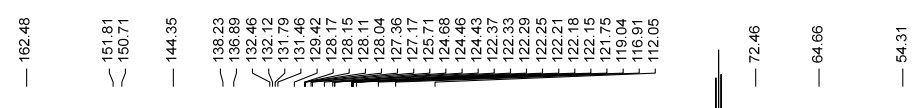

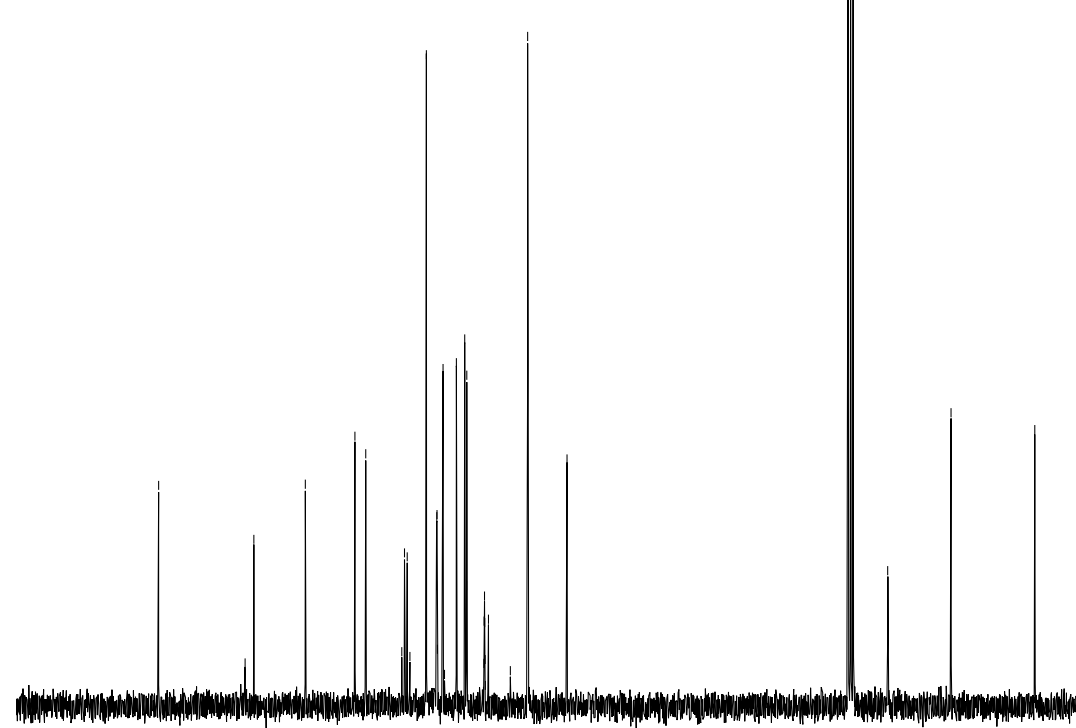

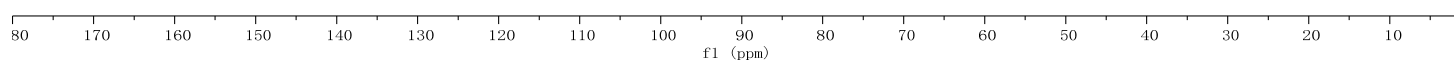


||<smiles>CC[C@H]1C(C(=O)OCc2cc(C(F)(F)F)cc(C(F)(F)F)c2)=CN(C(C)=O)N1c1ccccc1</smiles>

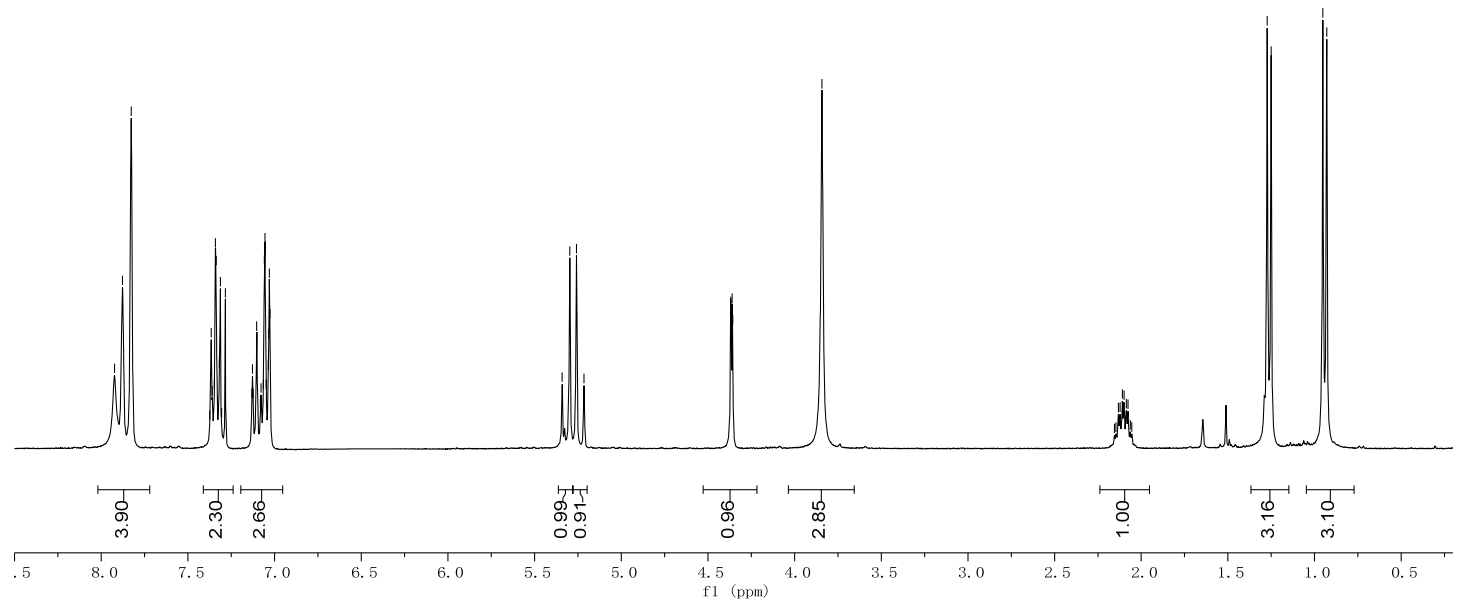
$\begin{array}{lll}0 & 0 \\ 0 & 0\end{array}$

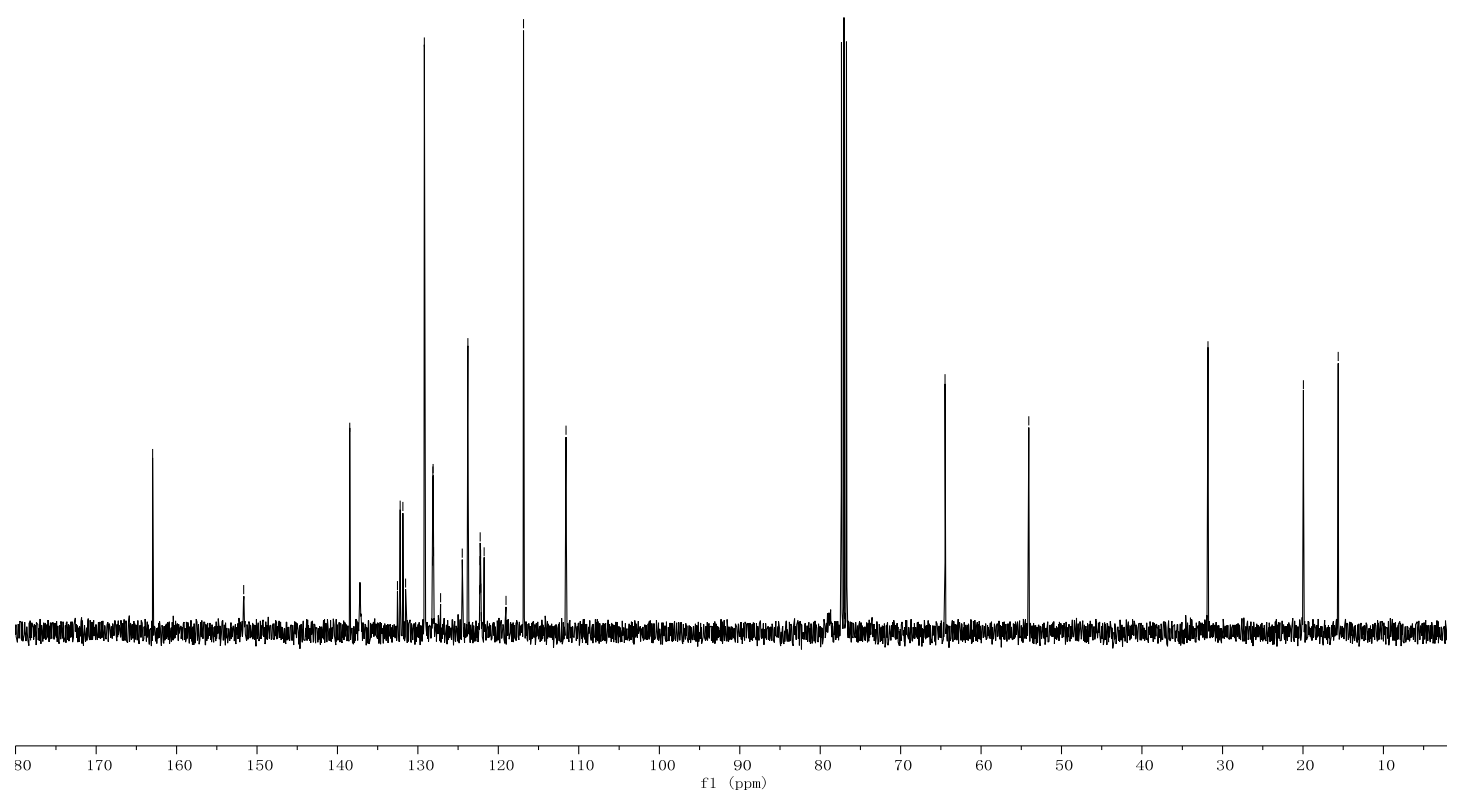




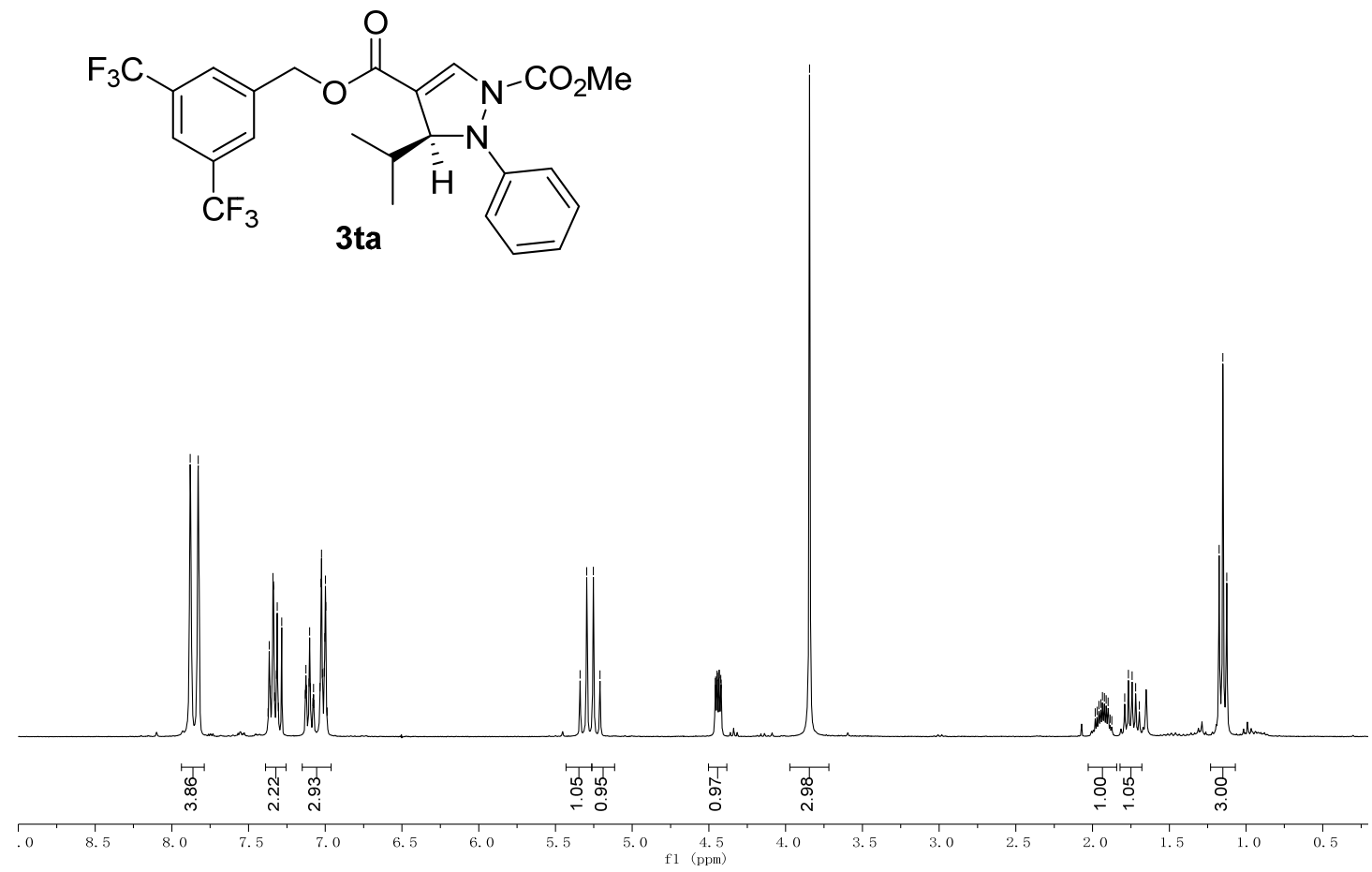

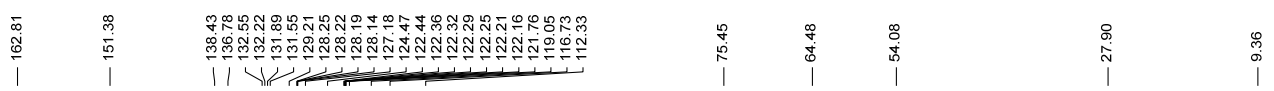

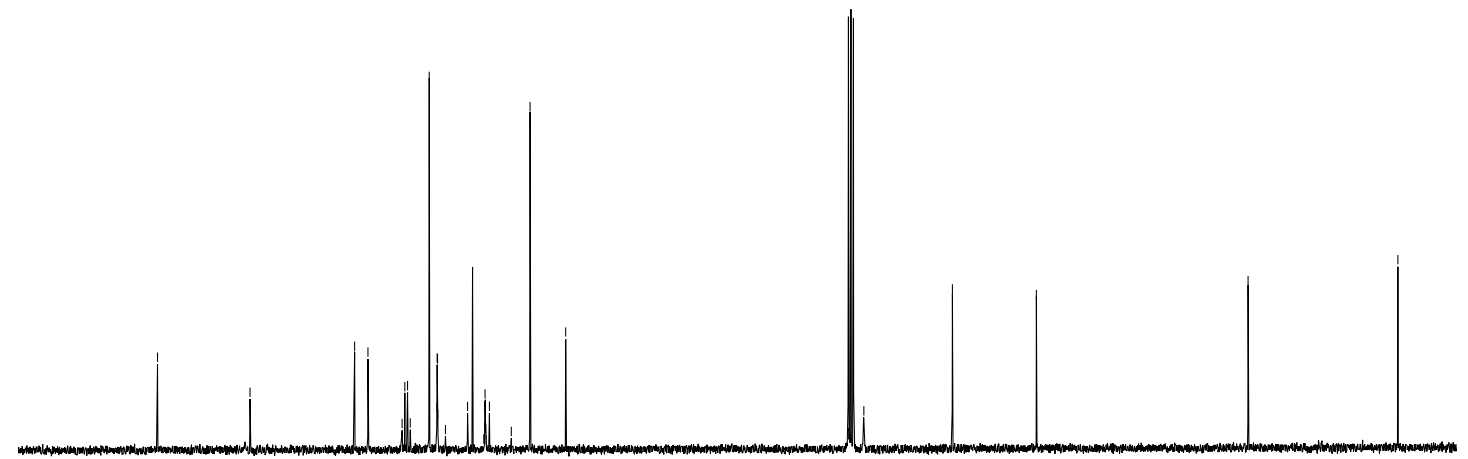

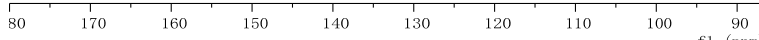


<smiles>CC(=O)N1C=C(C(=O)OCc2cc(C(F)(F)F)cc(C(F)(F)F)c2)[C@H](c2ccccc2)[C@H]1c1ccccc1F</smiles>

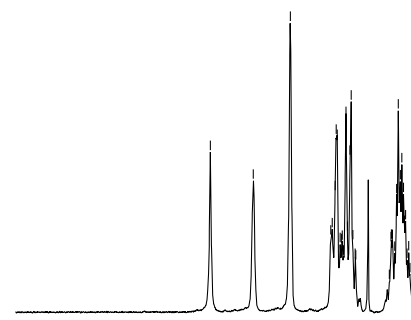

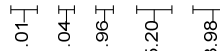

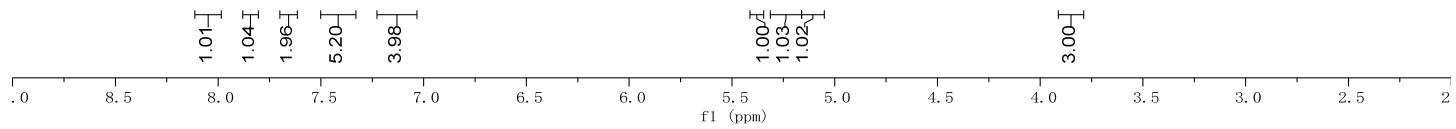

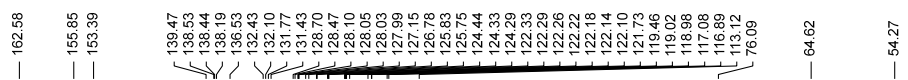
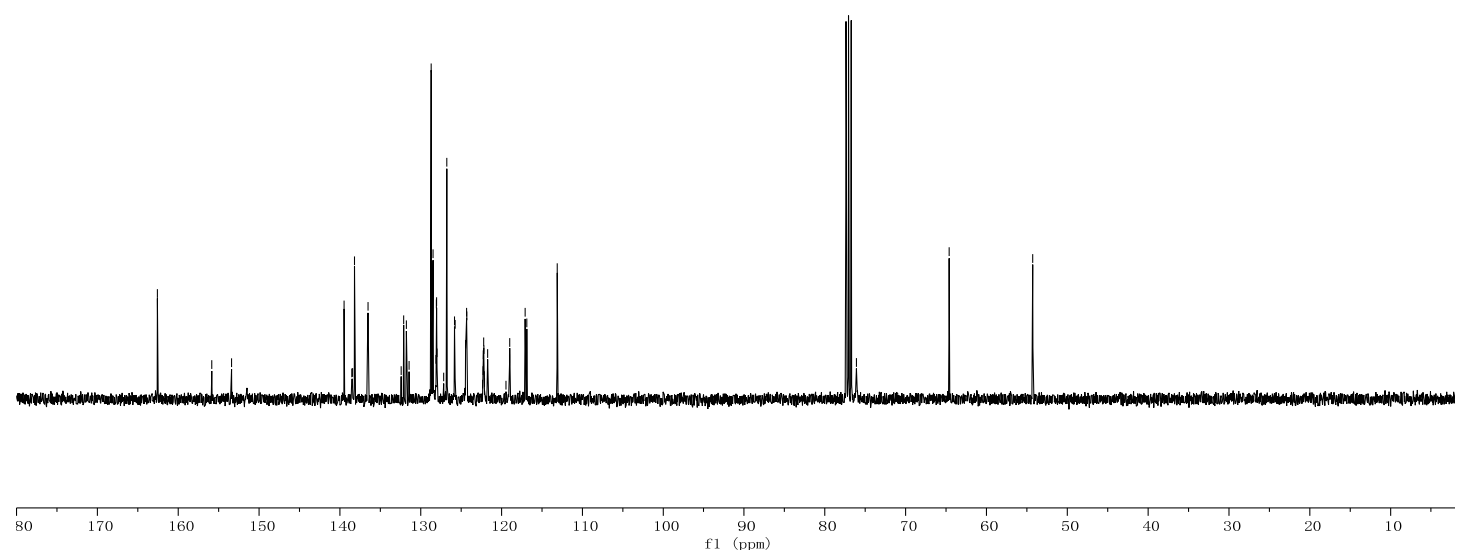


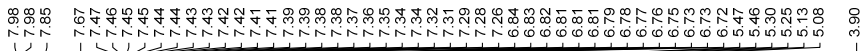<smiles>CC(=O)N1C=C(C(=O)OCc2cc(C(F)(F)F)cc(C(F)(F)F)c2)[C@H](c2ccccc2)N1c1cccc(F)c1</smiles>

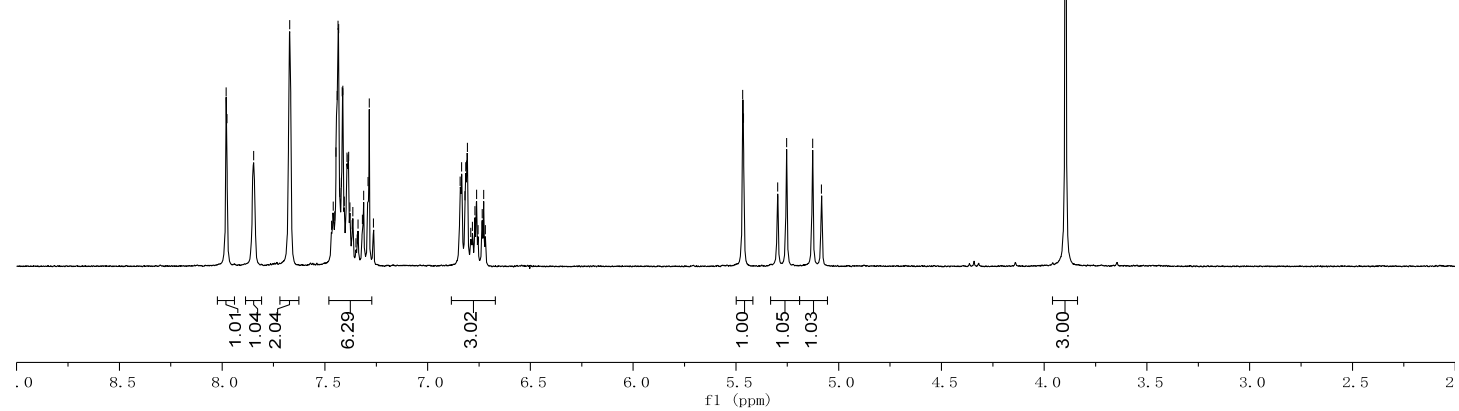

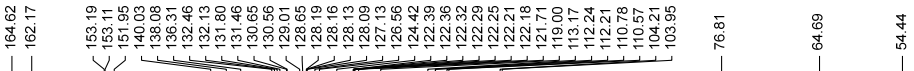
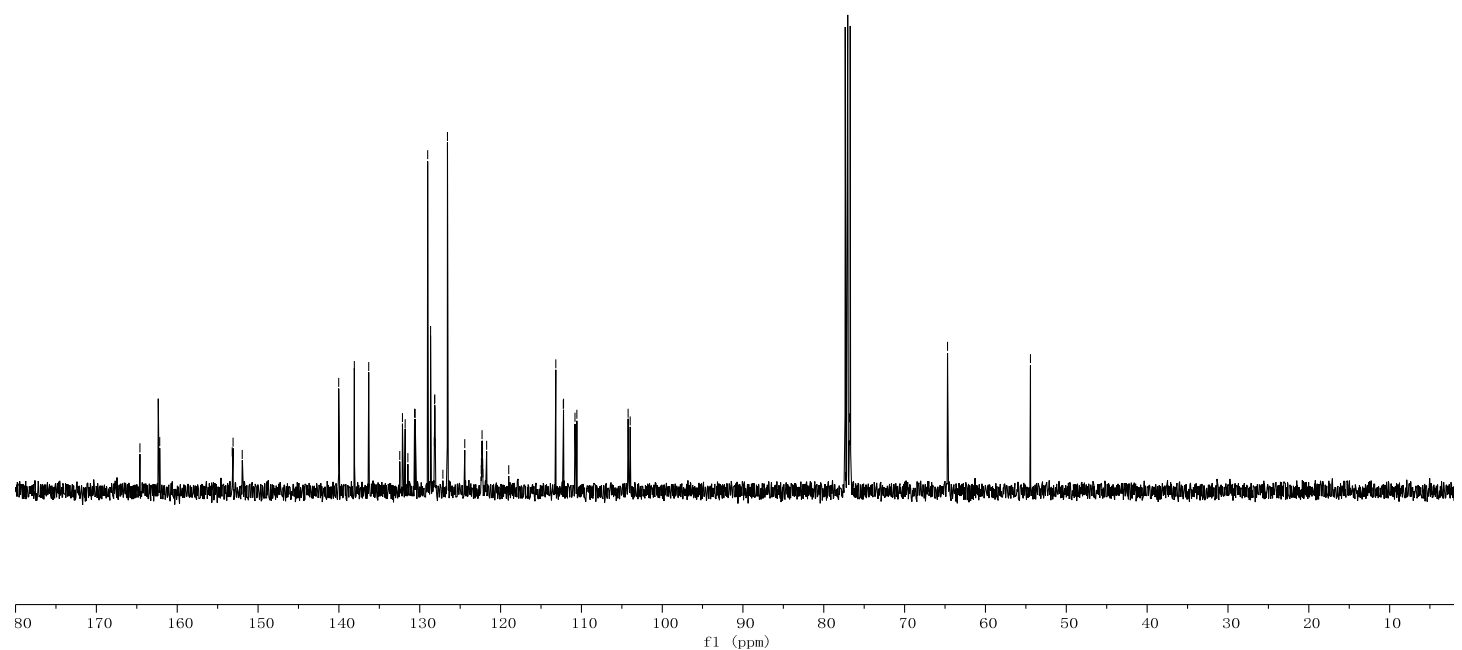
<smiles>CC(=O)N1C=C(C(=O)OCc2cc(C(F)(F)F)cc(C(F)(F)F)c2)C(c2ccccc2)[C@H]1c1ccc(F)cc1</smiles>

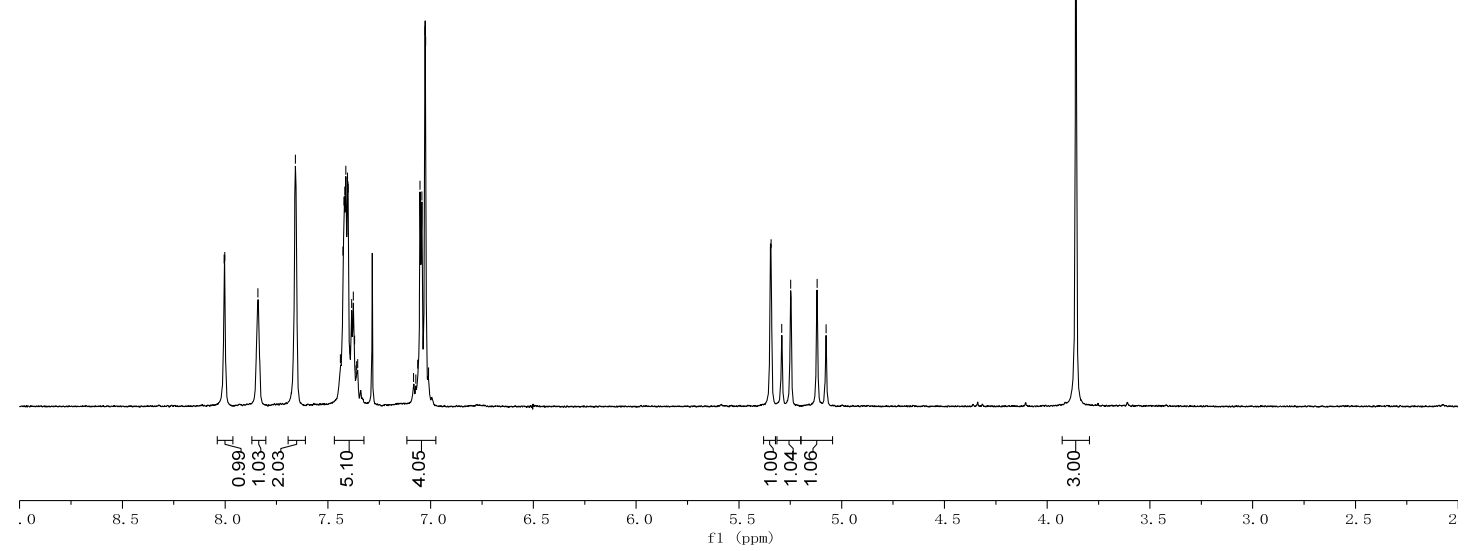

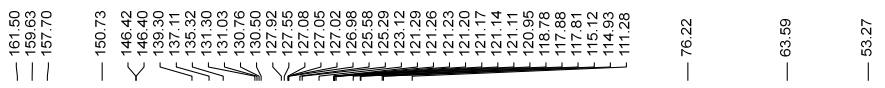

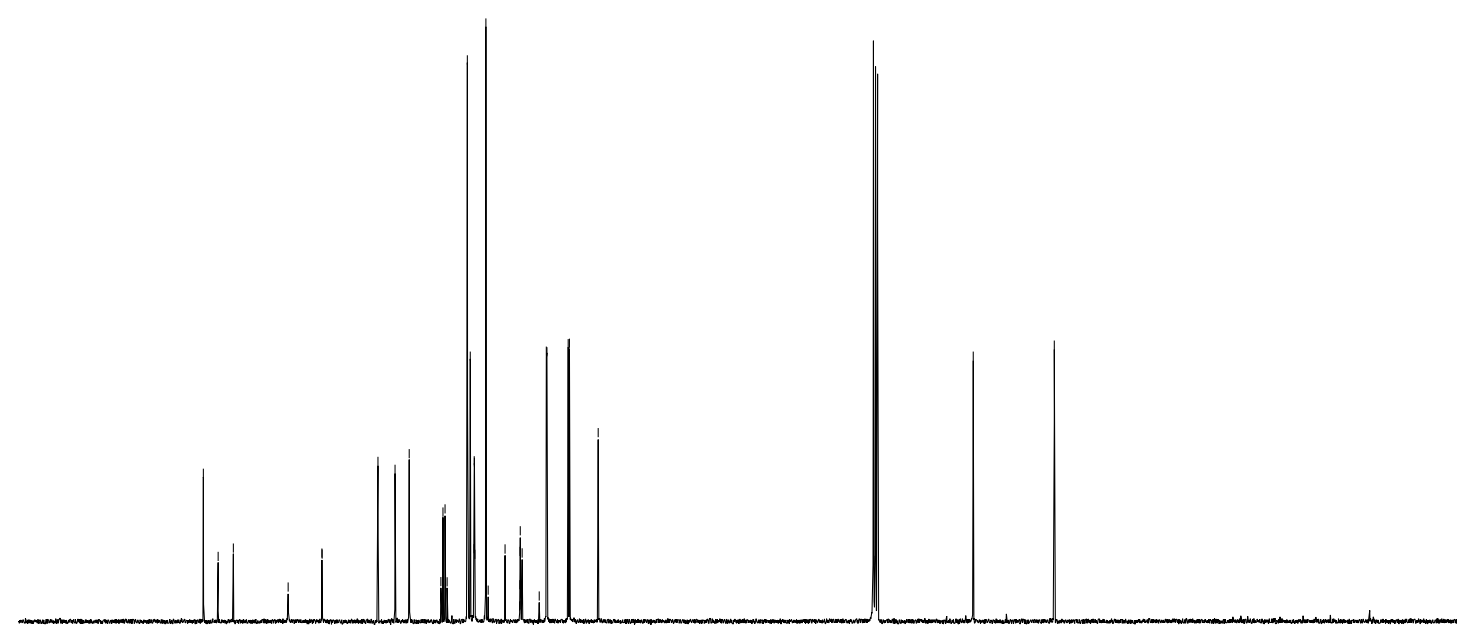




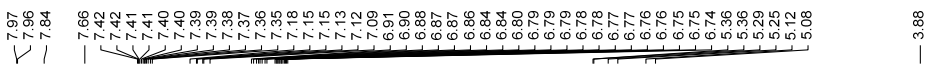

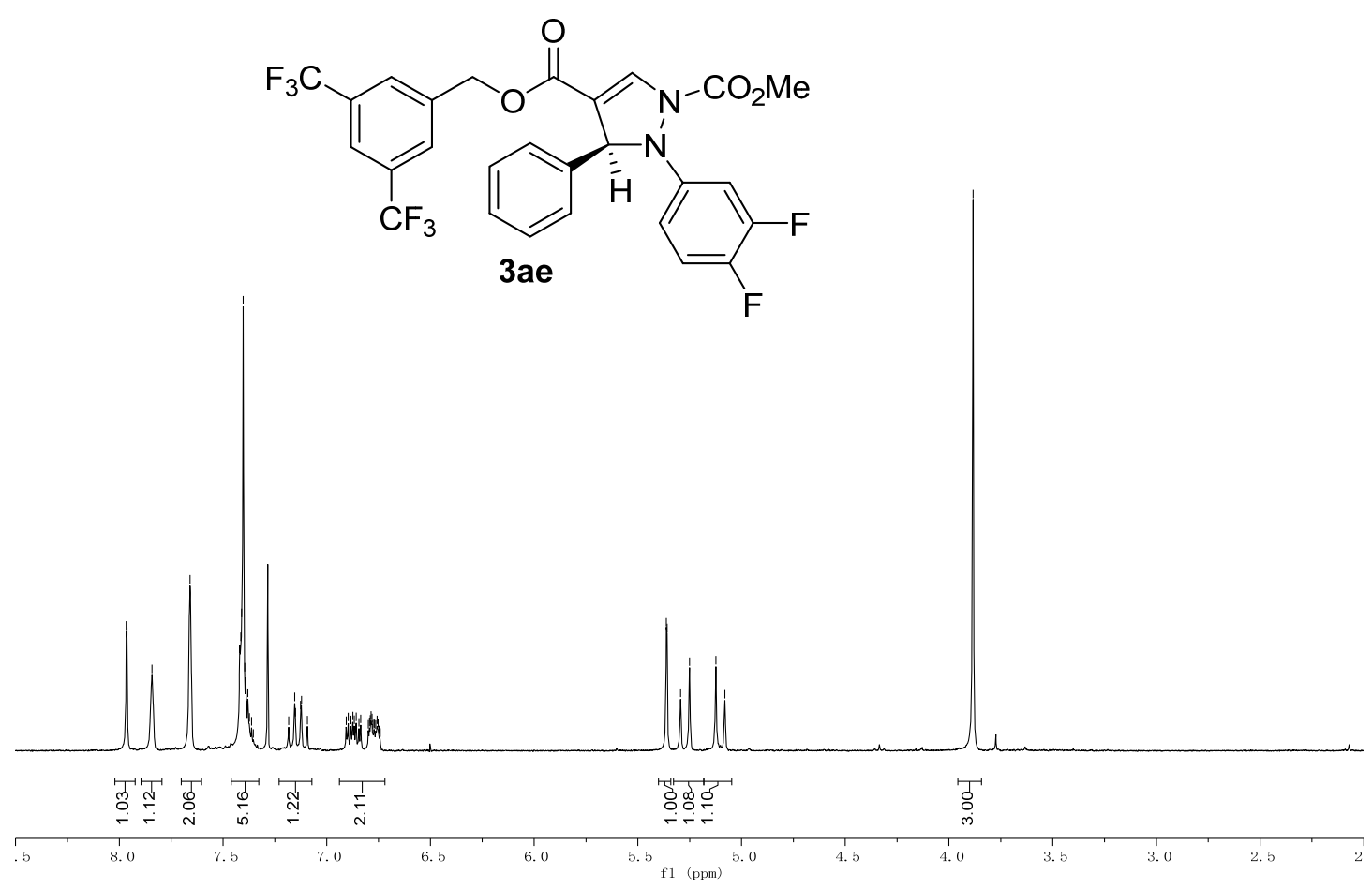

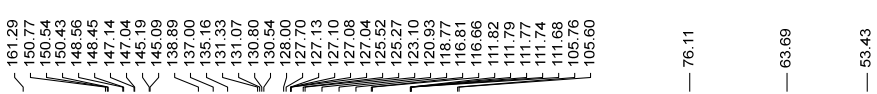

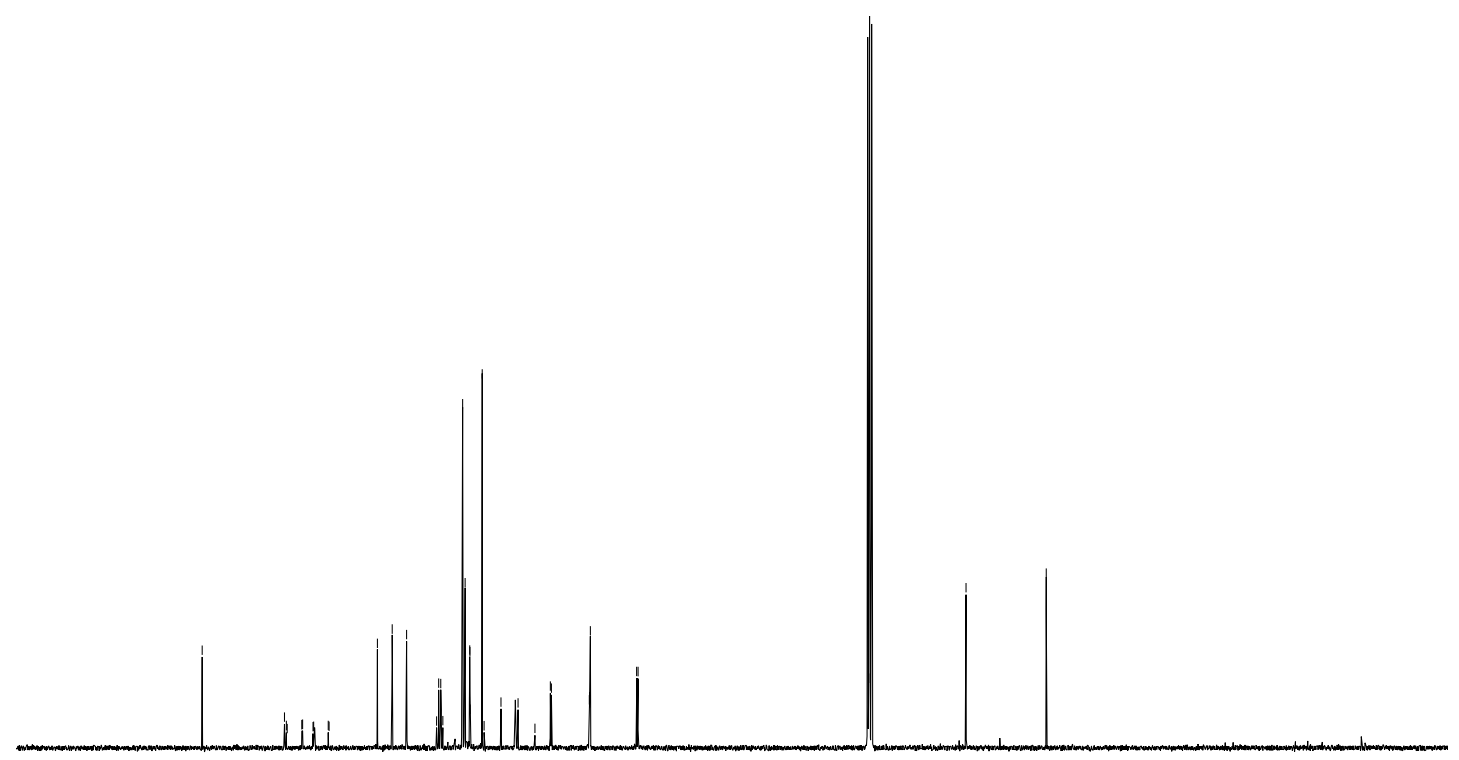

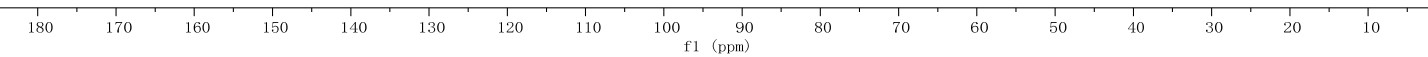




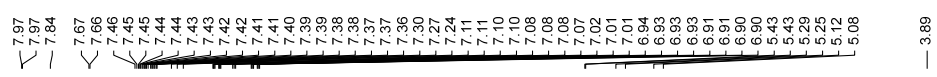<smiles>CC(=O)N1C=C(C(=O)OCc2cc(C(F)(F)F)cc(C(F)(F)F)c2)[C@@H](c2ccccc2)N1c1cccc(Cl)c1</smiles>
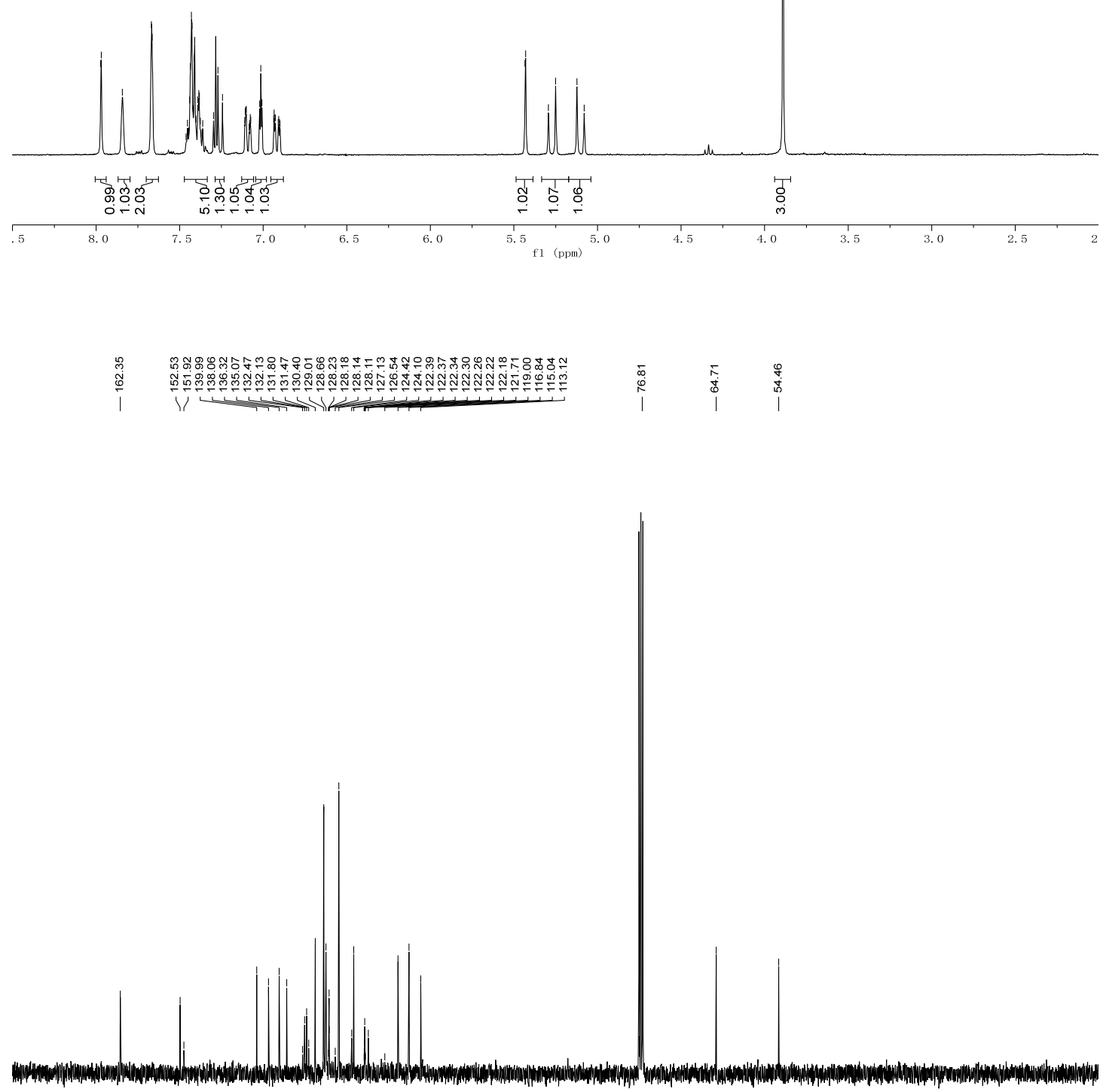

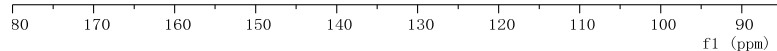


<smiles>CC(=O)N1C=C(C(=O)OCc2cc(C(F)(F)F)cc(C(F)(F)F)c2)[C@H](c2ccccc2)[C@H]1c1ccc(Cl)cc1</smiles>

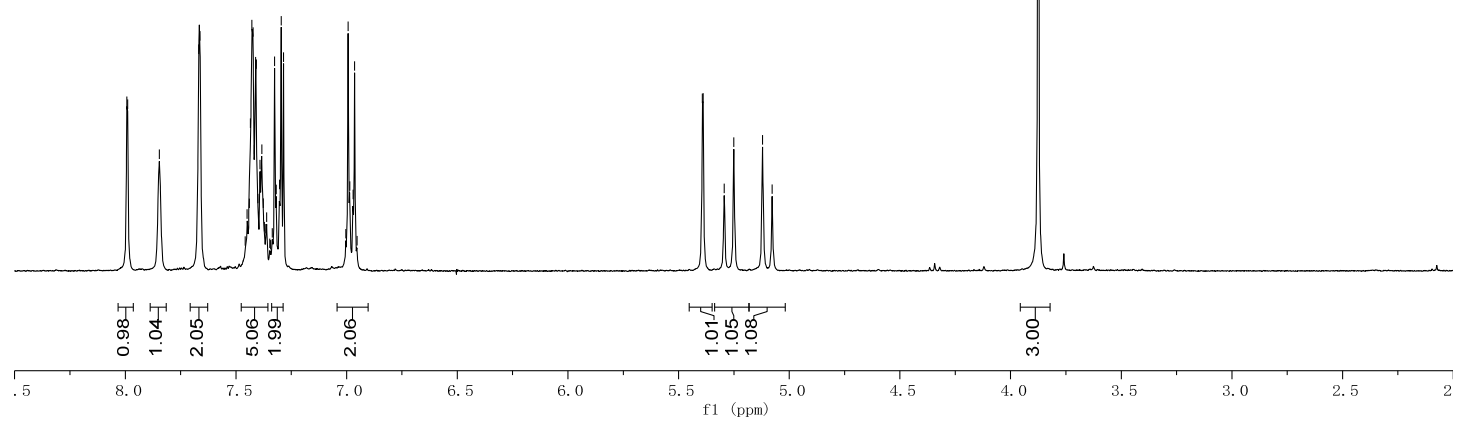

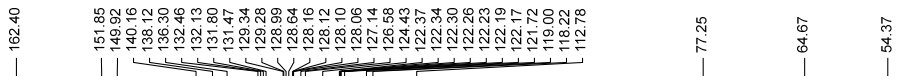
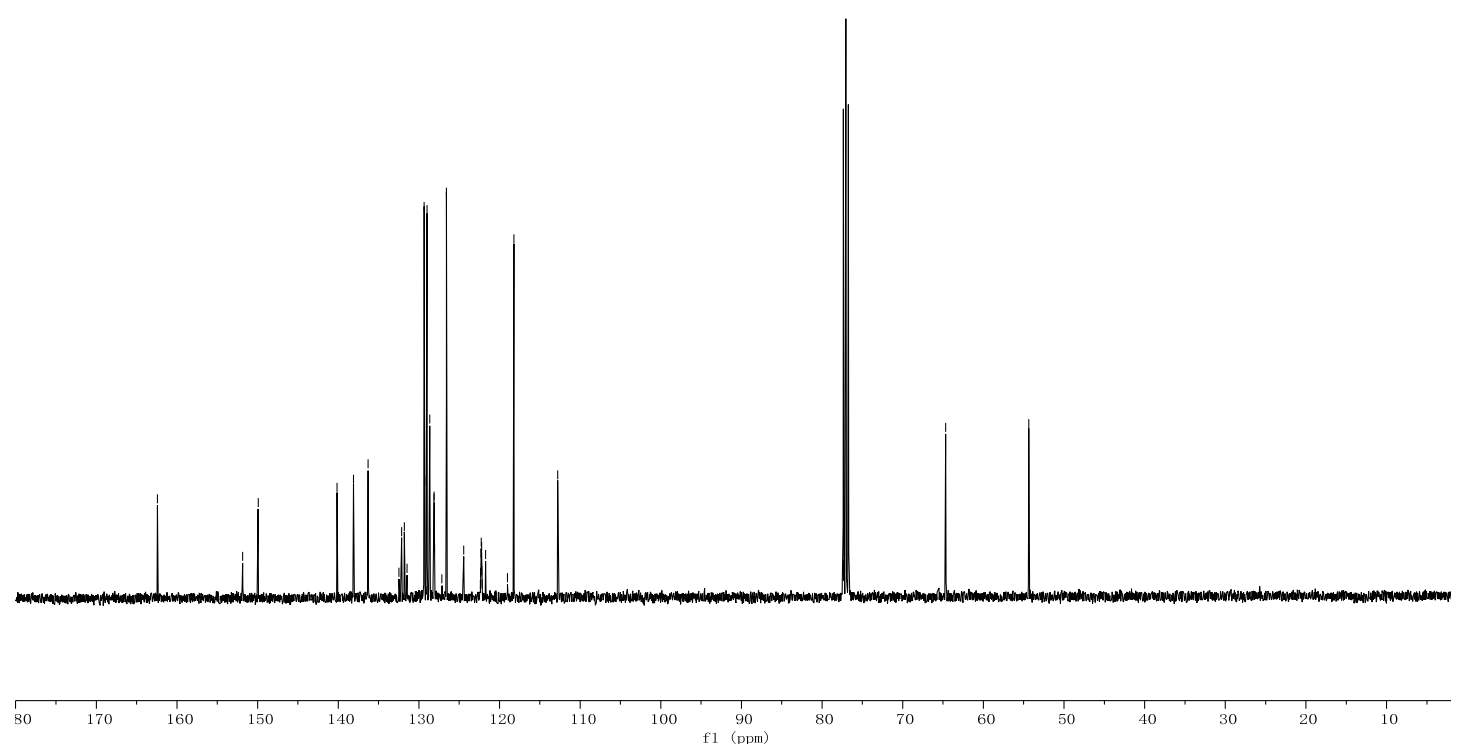
<smiles>CC(=O)N1C=C(C(=O)OCc2cc(C(F)(F)F)cc(C(F)(F)F)c2)[C@H](c2ccccc2)N1c1ccccc1Br</smiles>

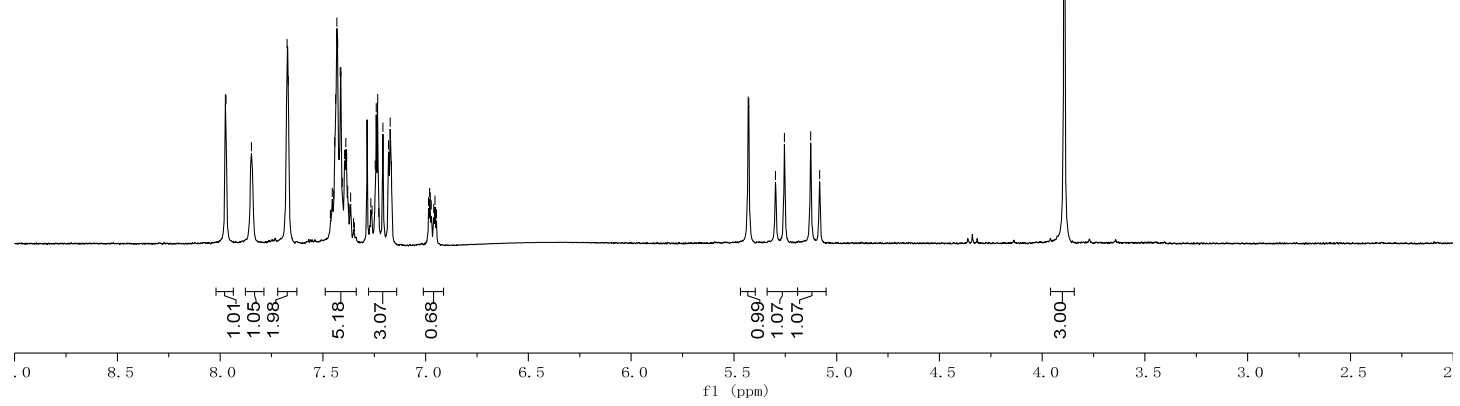

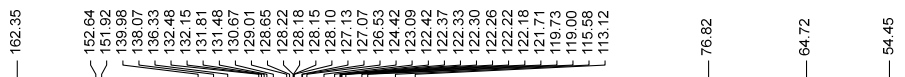
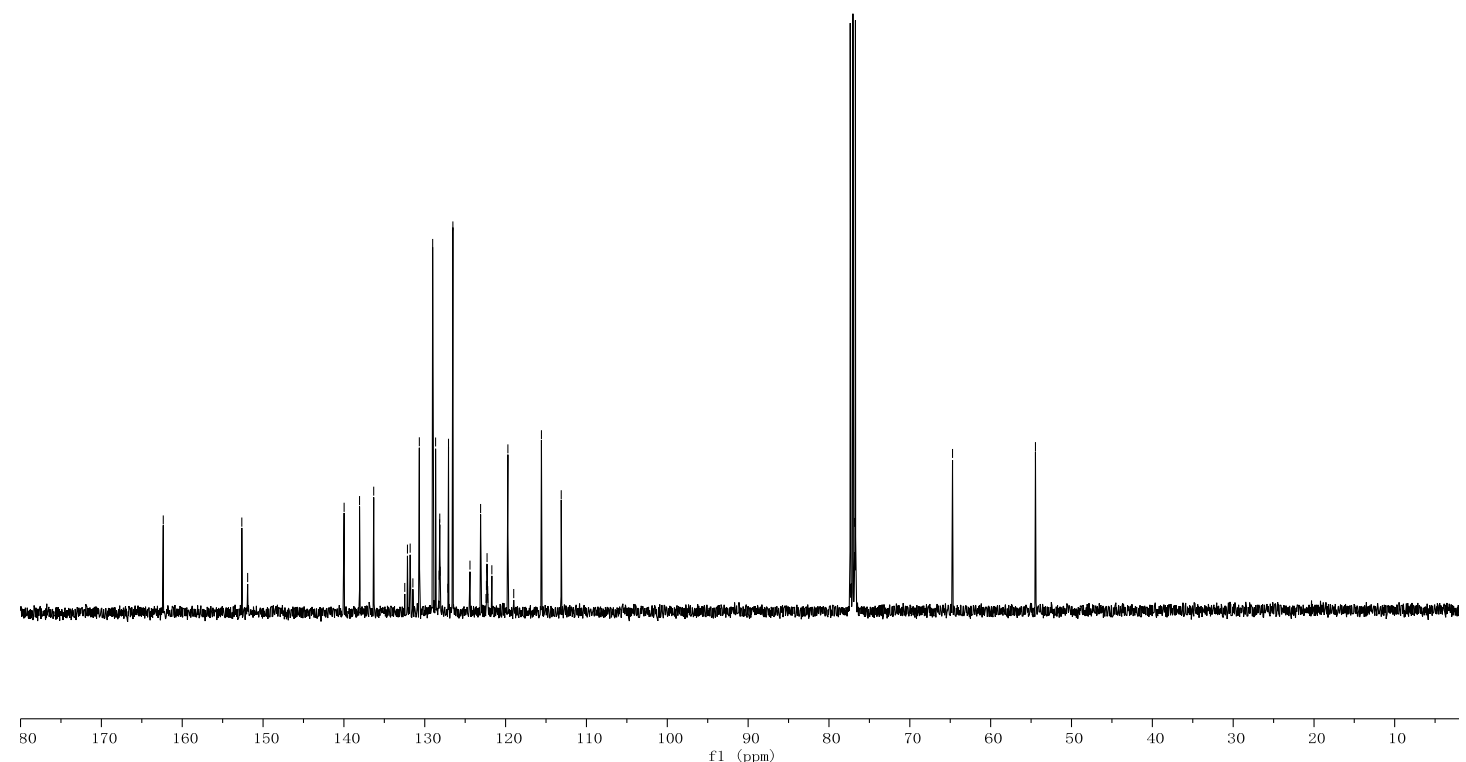
은

$\stackrel{\substack{\infty \\ i}}{i}$
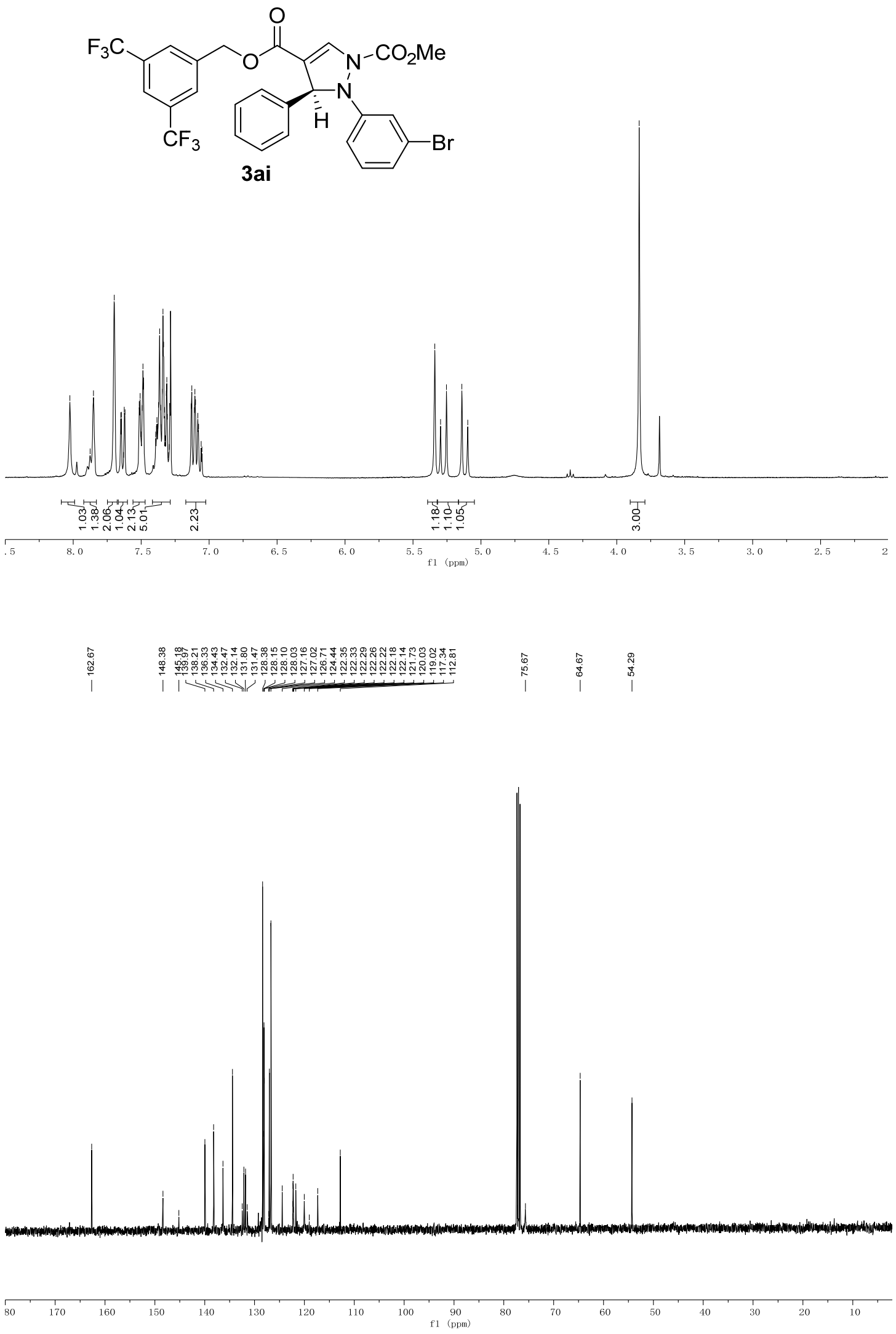

S74 


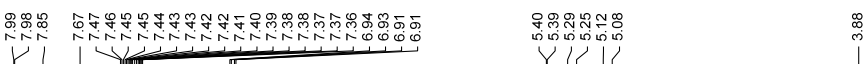<smiles>CC(=O)N1C=C(C(=O)OCc2cc(C(F)(F)F)cc(C(F)(F)F)c2)[C@H](c2ccccc2)N1c1ccc(Br)cc1</smiles>

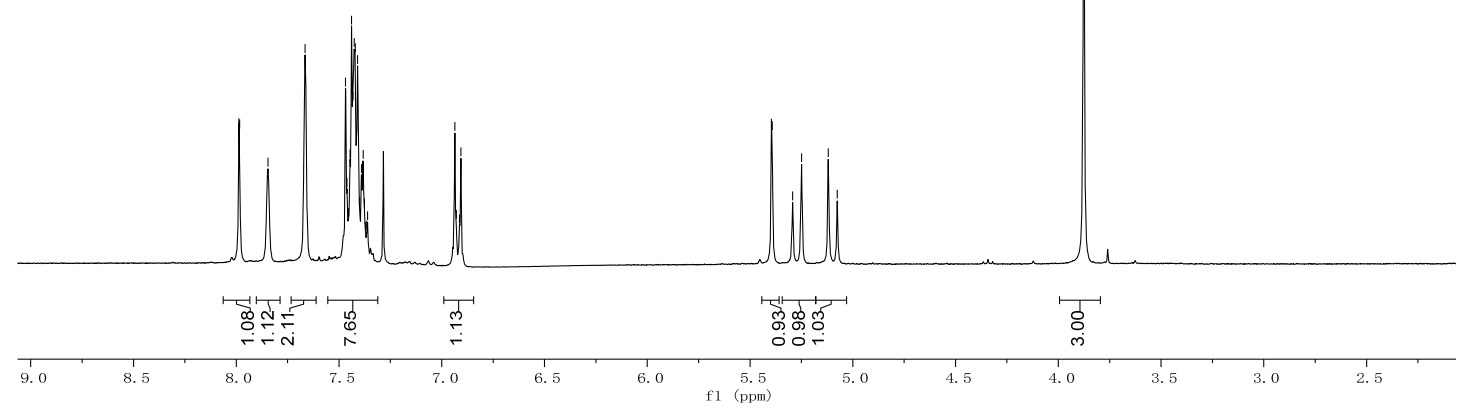

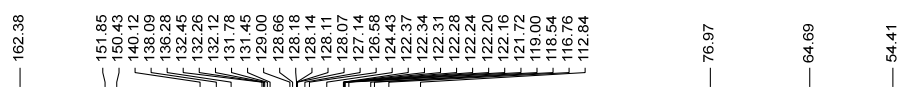

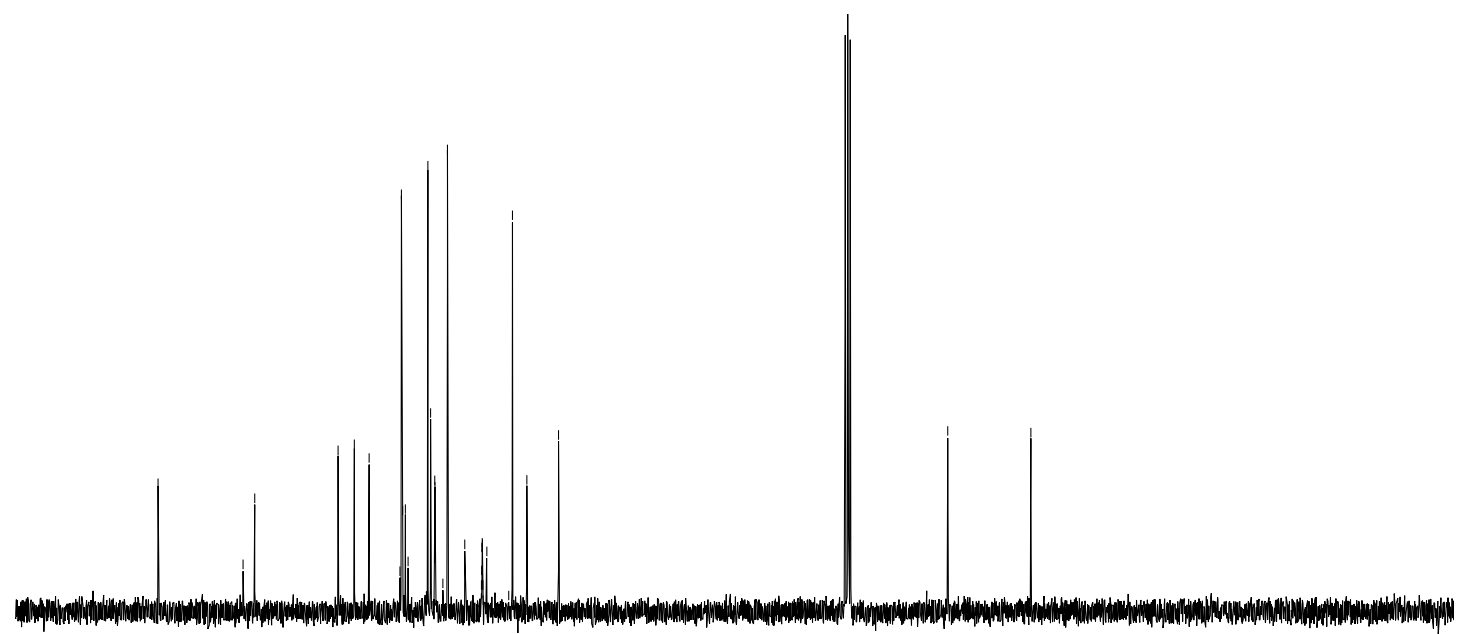

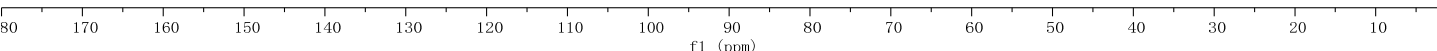


<smiles>CC(=O)N1C=C(C(=O)OCc2cc(C(F)(F)F)cc(C(F)(F)F)c2)[C@H](c2ccccc2)N1c1ccc(C(F)(F)F)cc1</smiles>

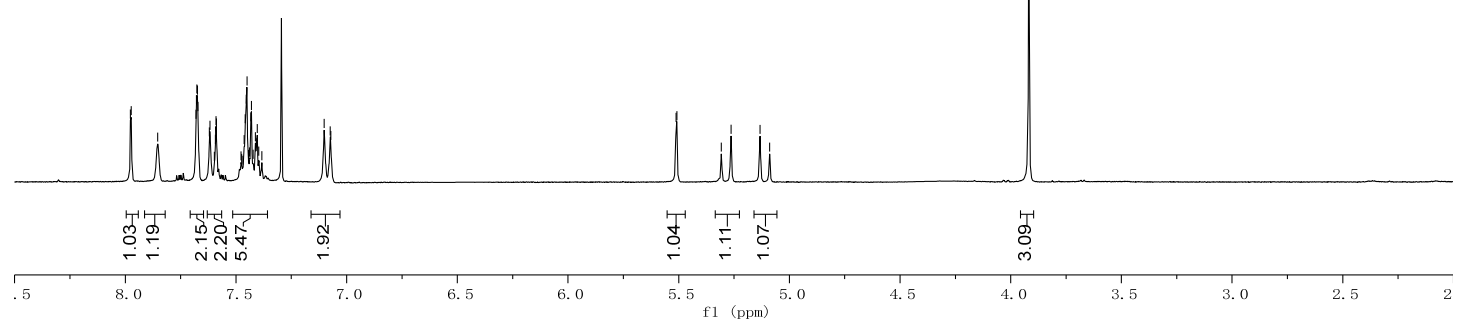

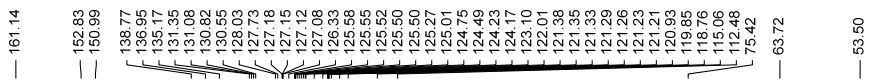

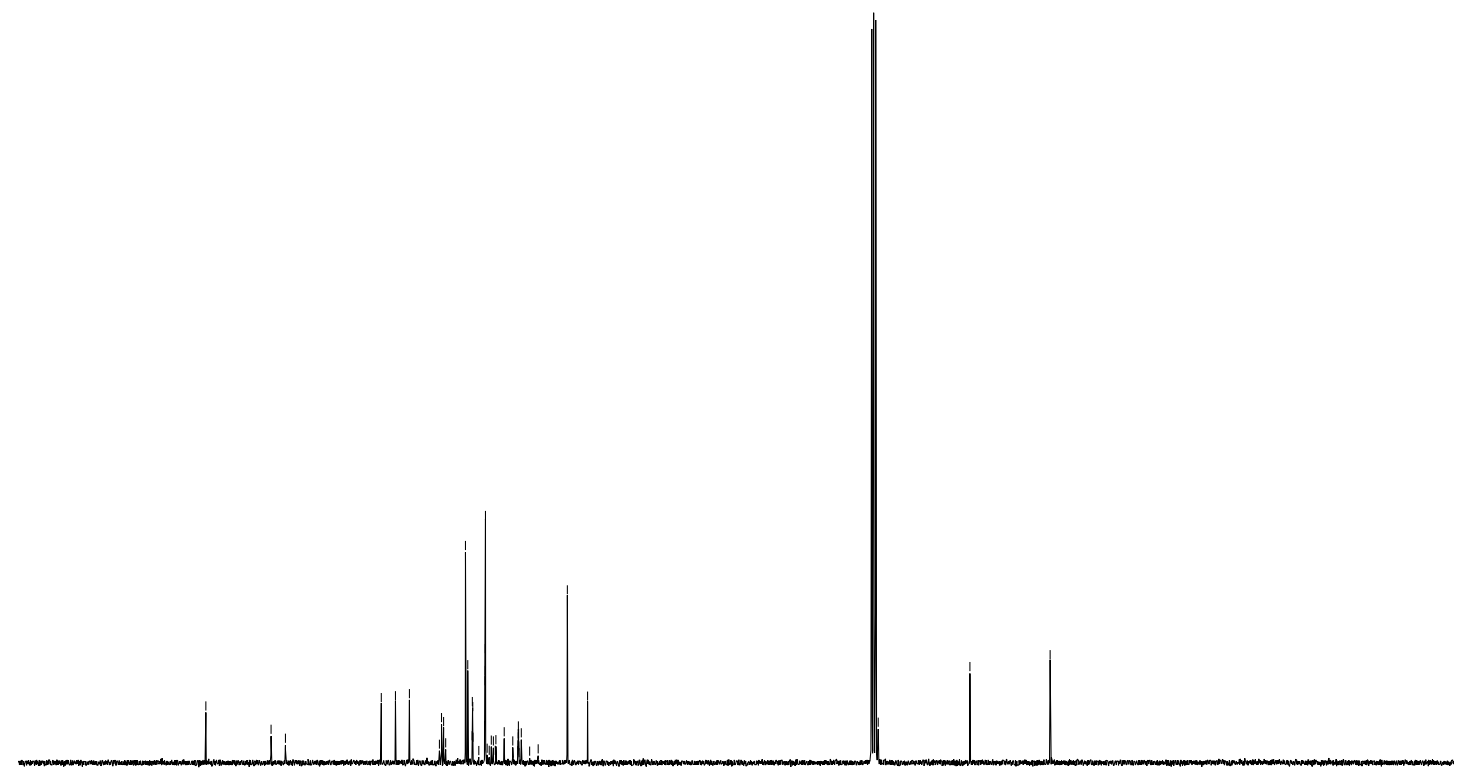

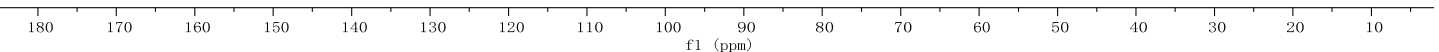


<smiles>CC(=O)N1C=C(C(=O)OCc2cc(C(F)(F)F)cc(C(F)(F)F)c2)[C@H](c2ccccc2)N1c1ccccc1C</smiles>
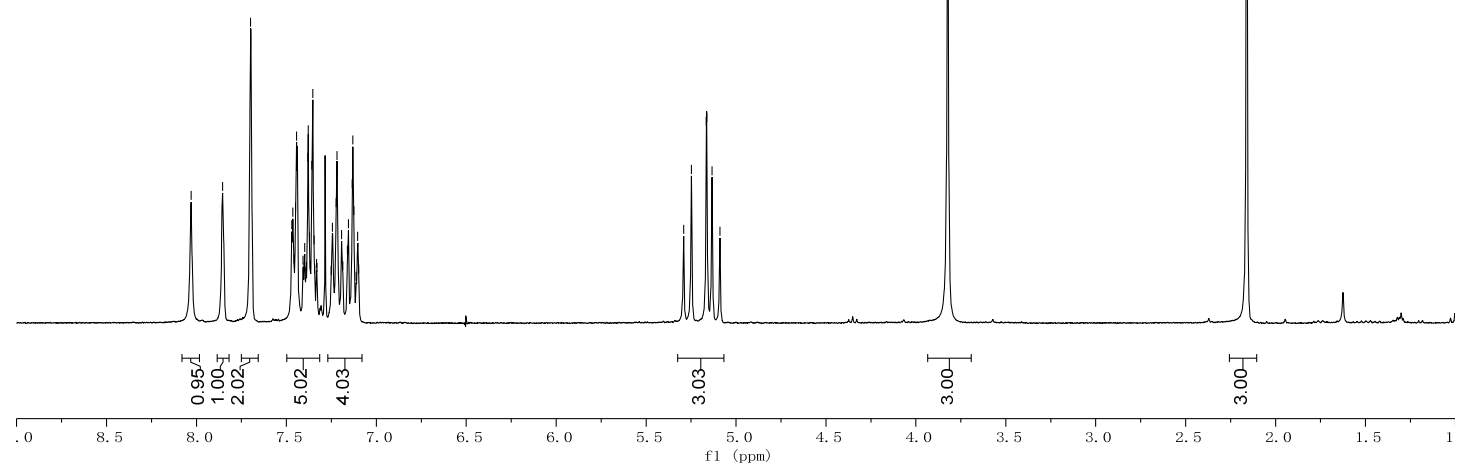

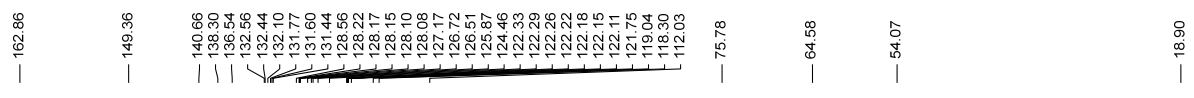

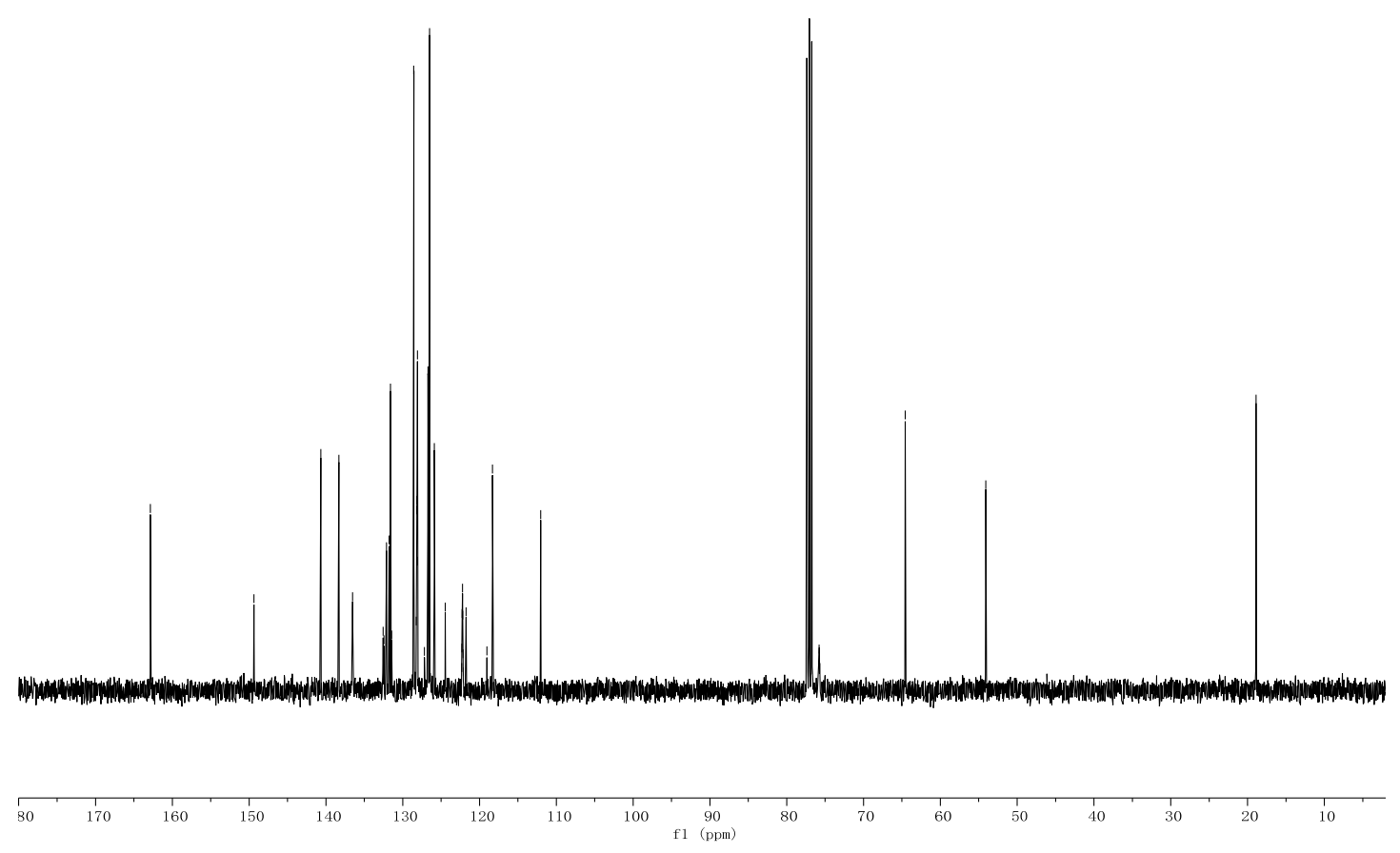



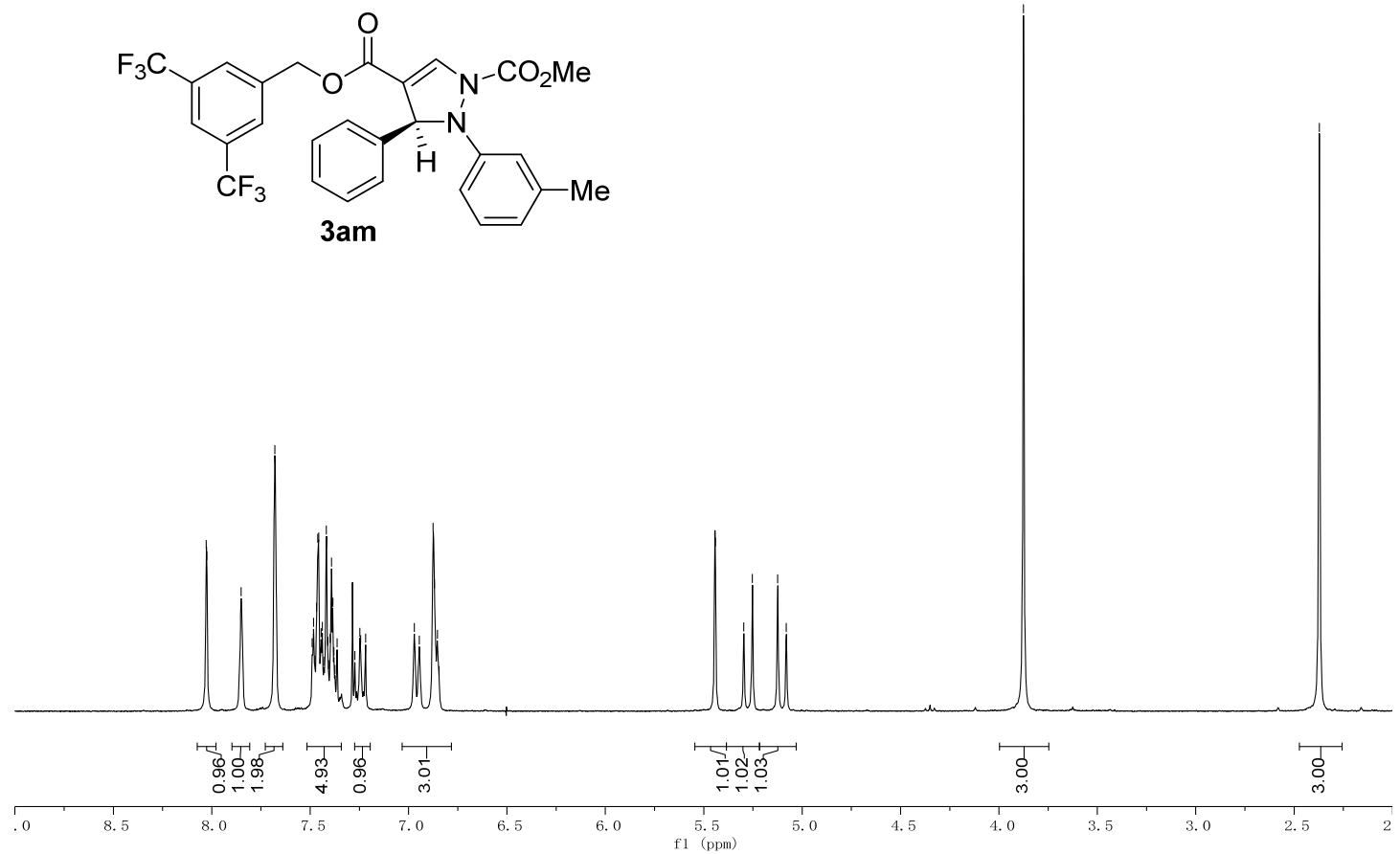

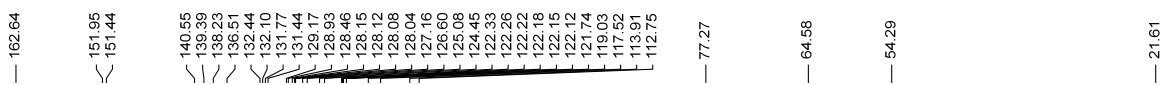

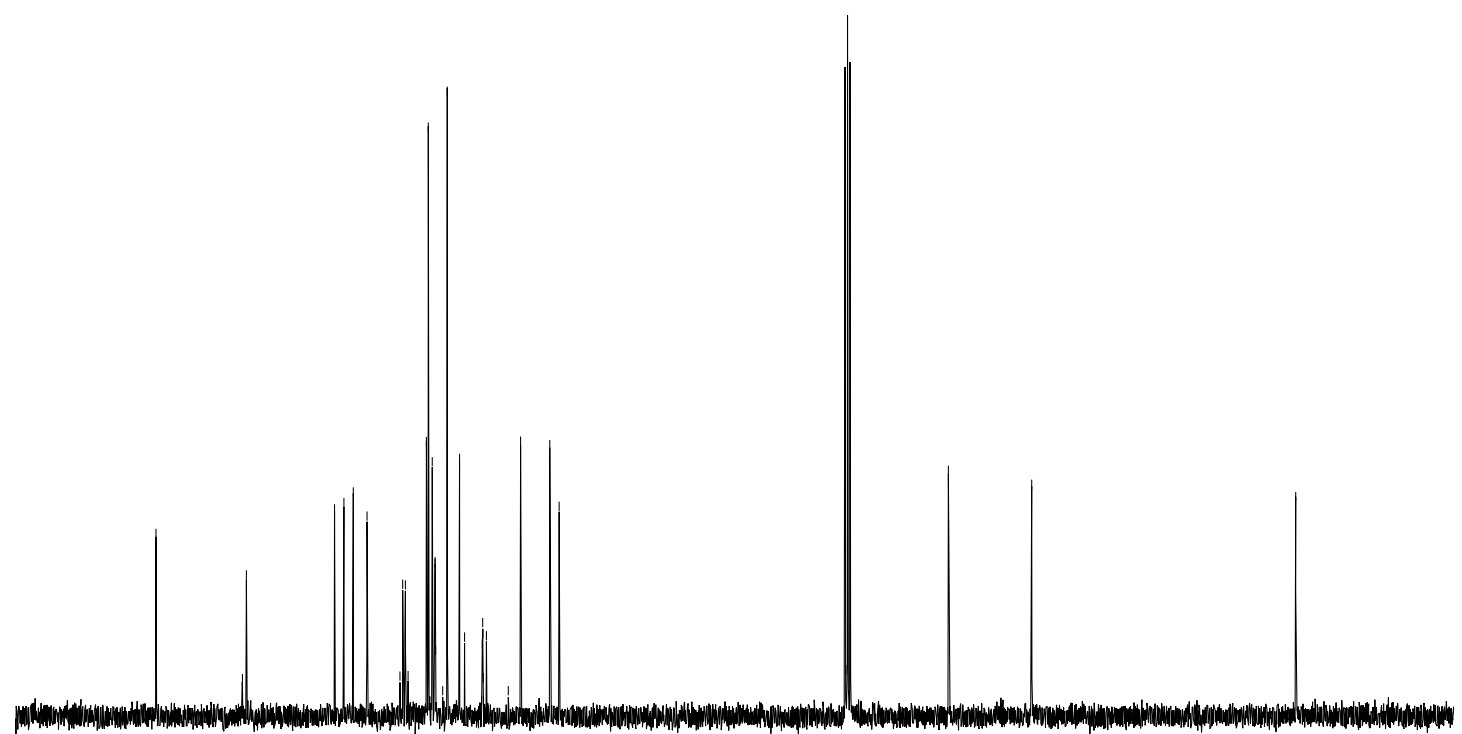

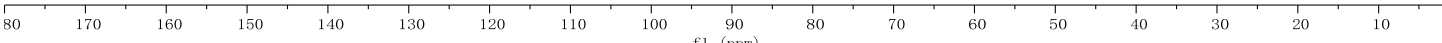


Y)<smiles>CC(=O)N1C=C(C(=O)OCc2cc(C(F)(F)F)cc(C(F)(F)F)c2)C(c2ccccc2)N1c1ccc(C)cc1</smiles>

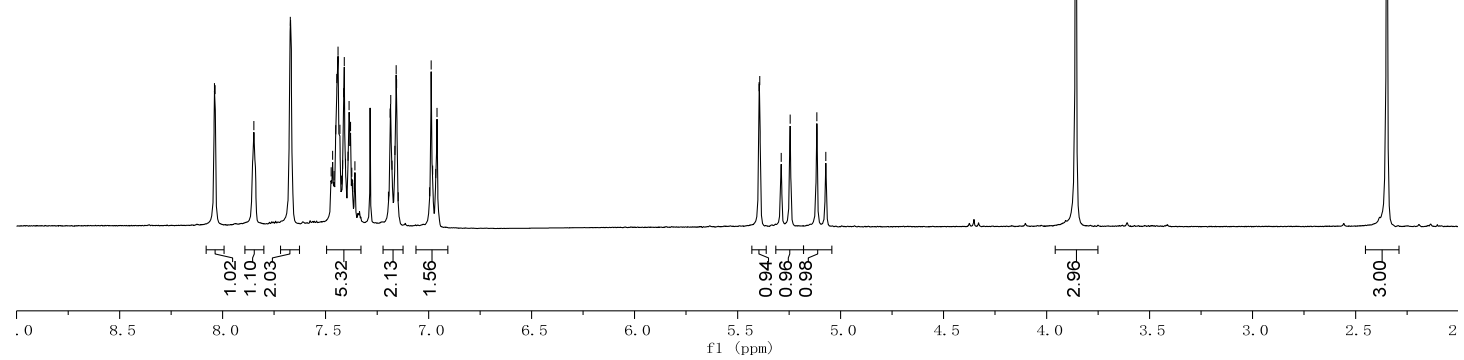

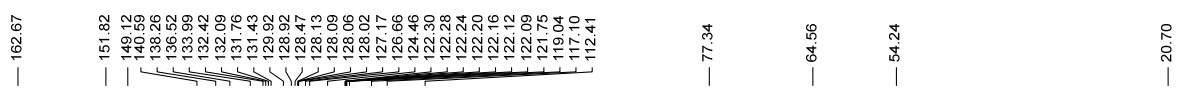

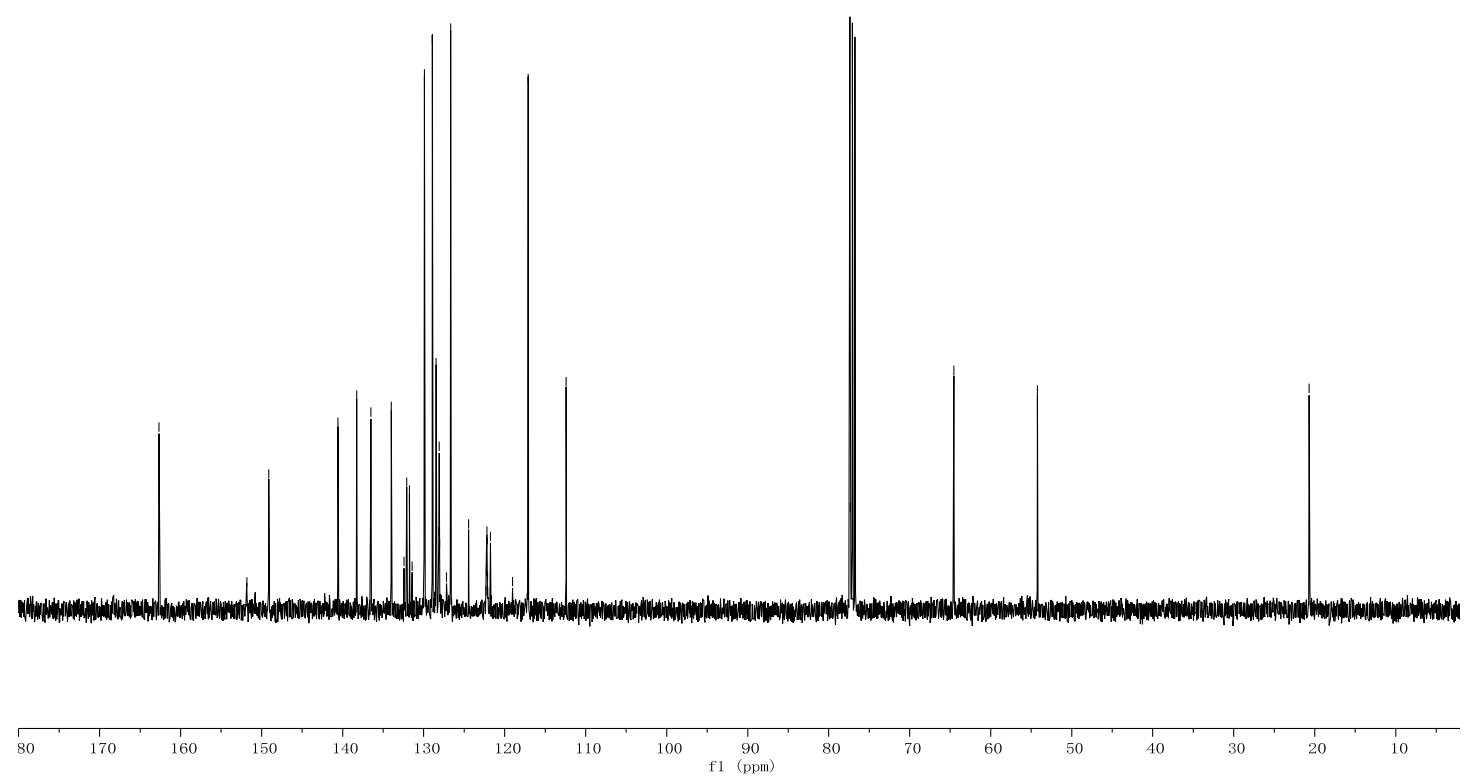


<smiles>CC(=O)N1C=C(C(=O)OCc2cc(C(F)(F)F)cc(C(F)(F)F)c2)[C@H](c2ccccc2)N1c1ccc(C)cc1C</smiles>
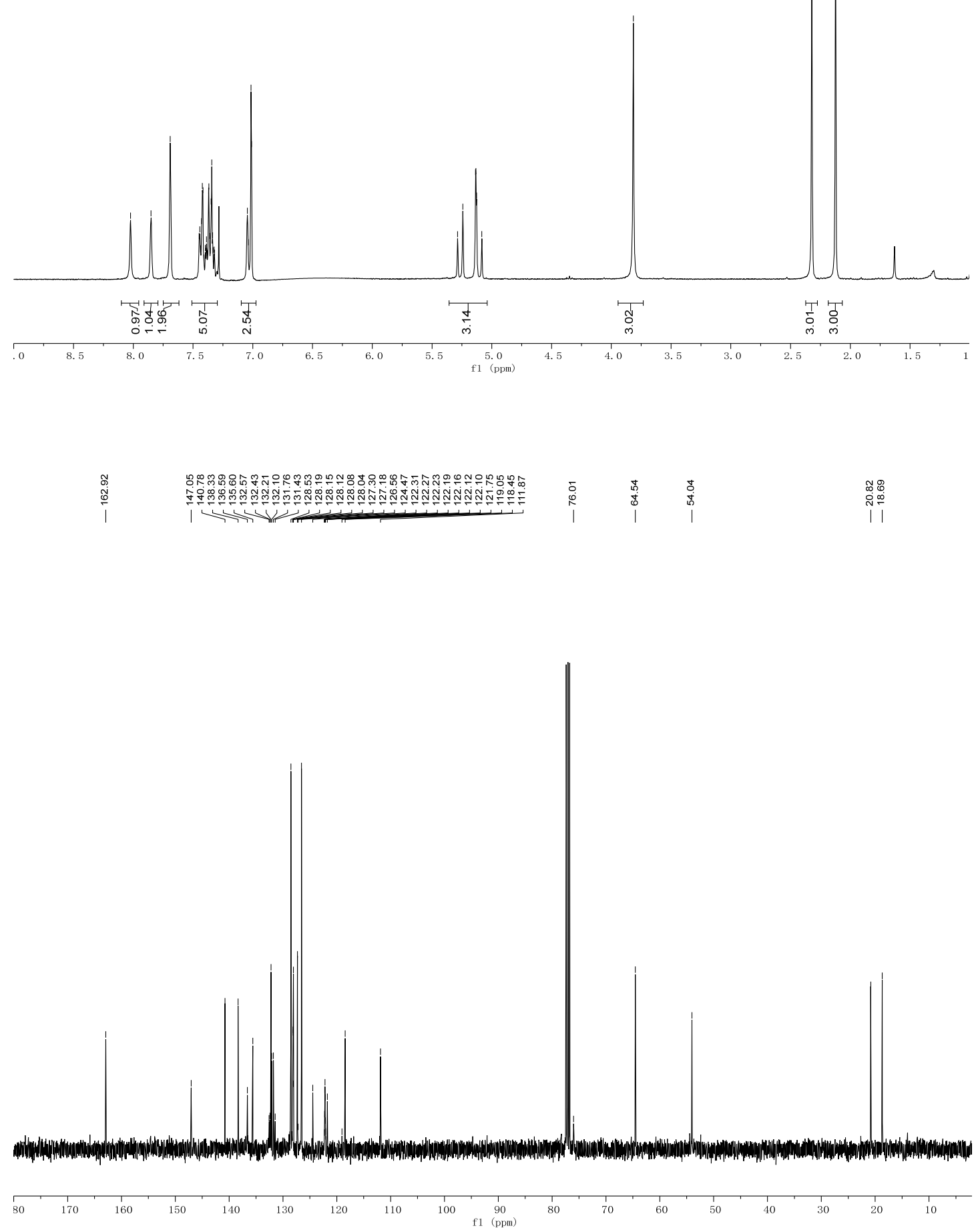
<smiles></smiles>
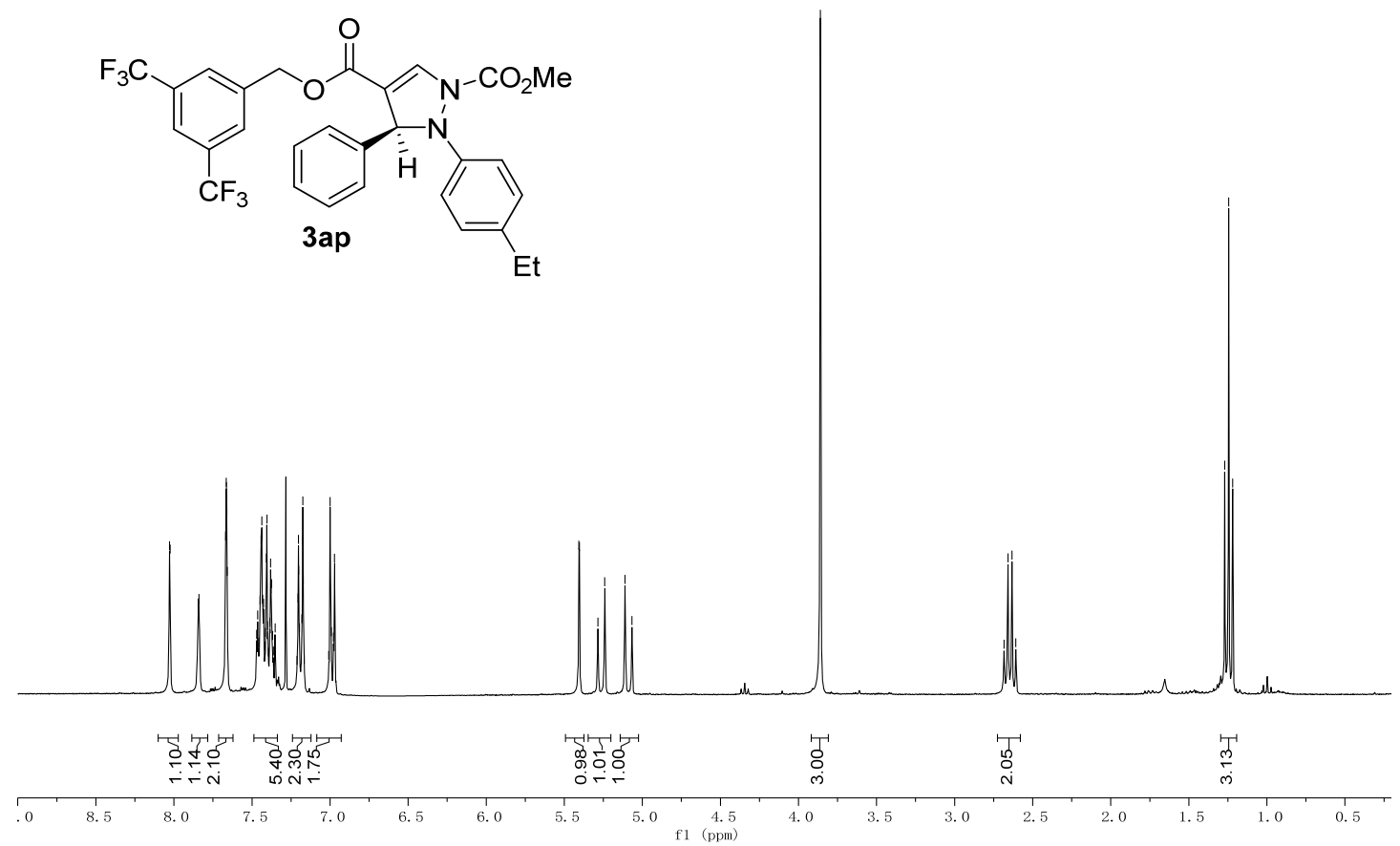

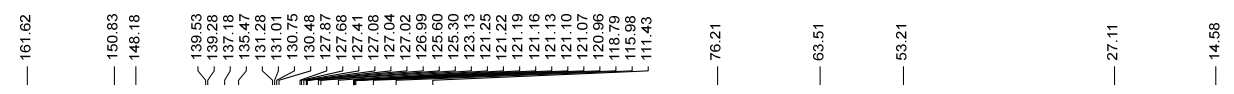

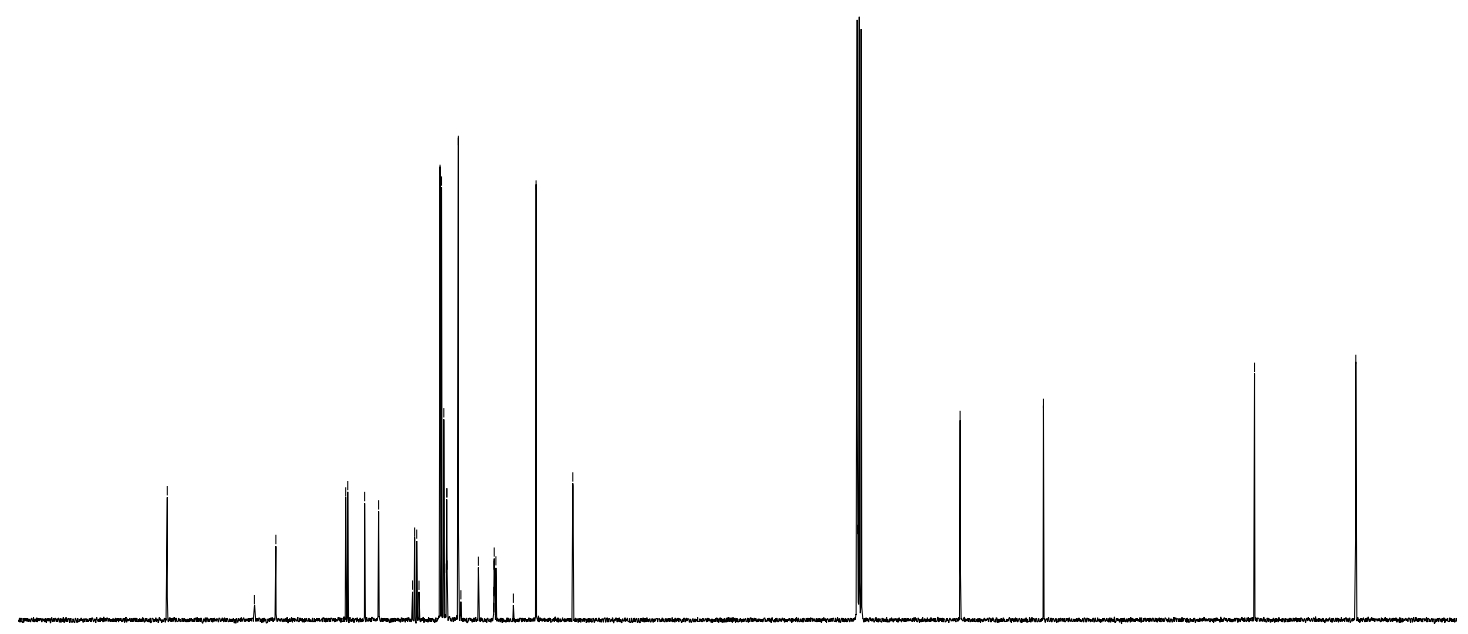

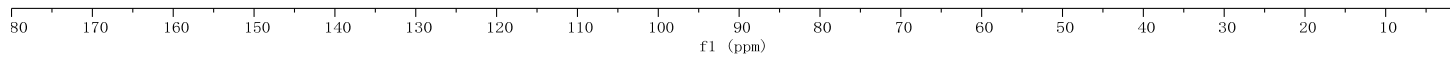




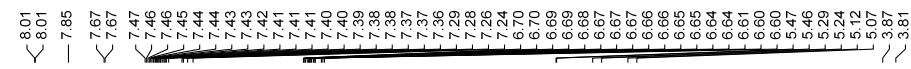<smiles></smiles>

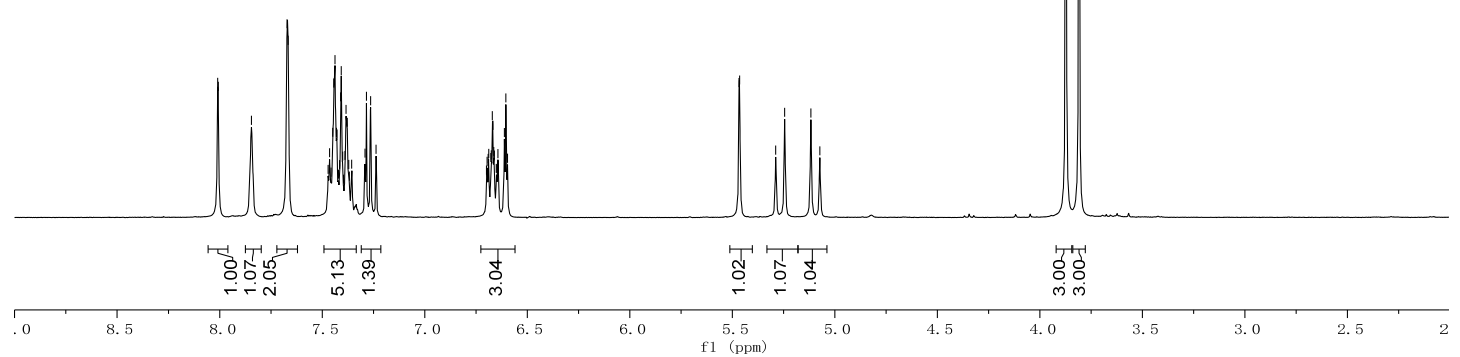

|l |l

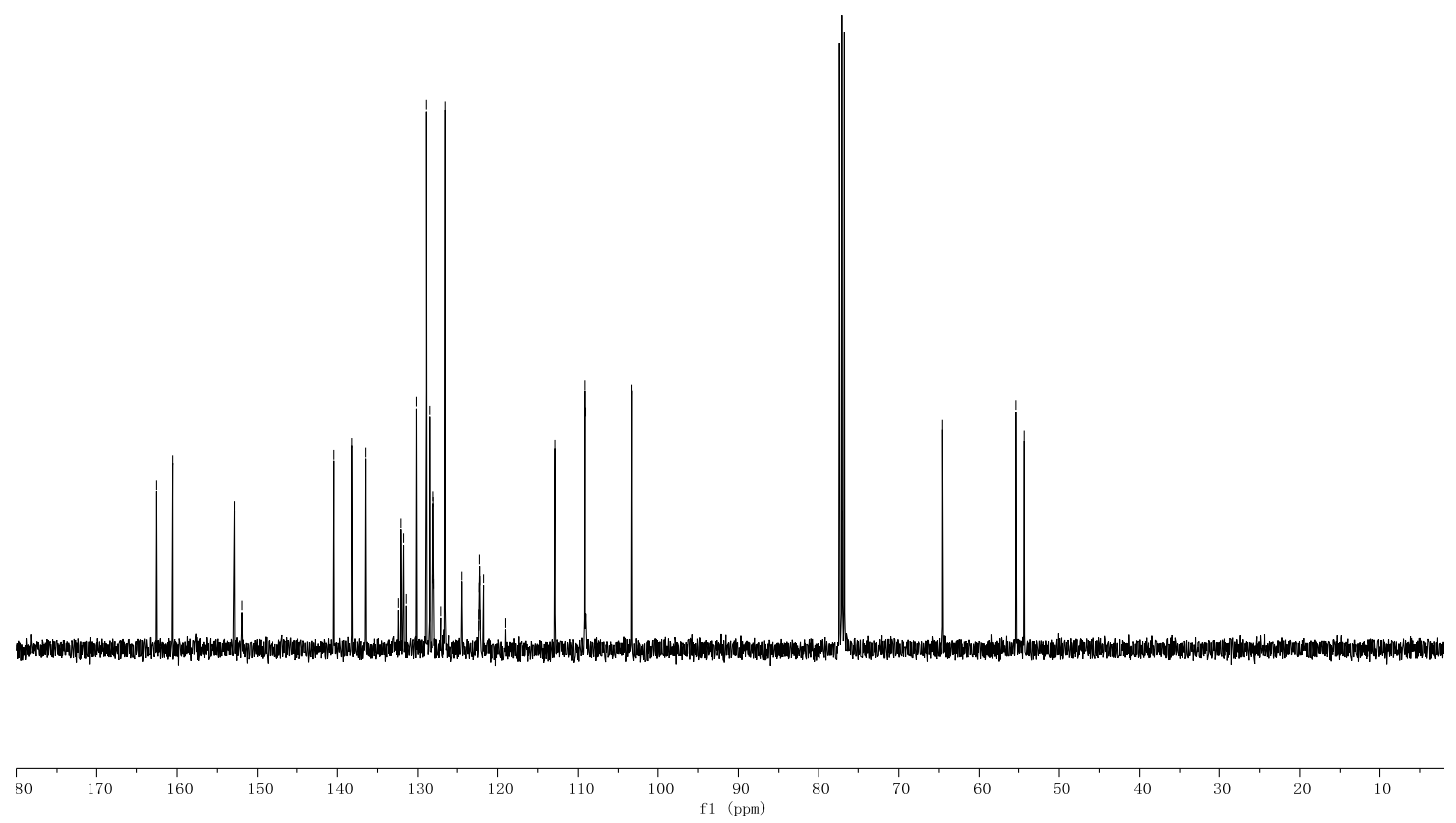


<smiles>COc1ccc(N2C(C(=O)OCc3cc(C(F)(F)F)cc(C(F)(F)F)c3)=CN(C(C)=O)C2c2ccccc2)cc1</smiles>

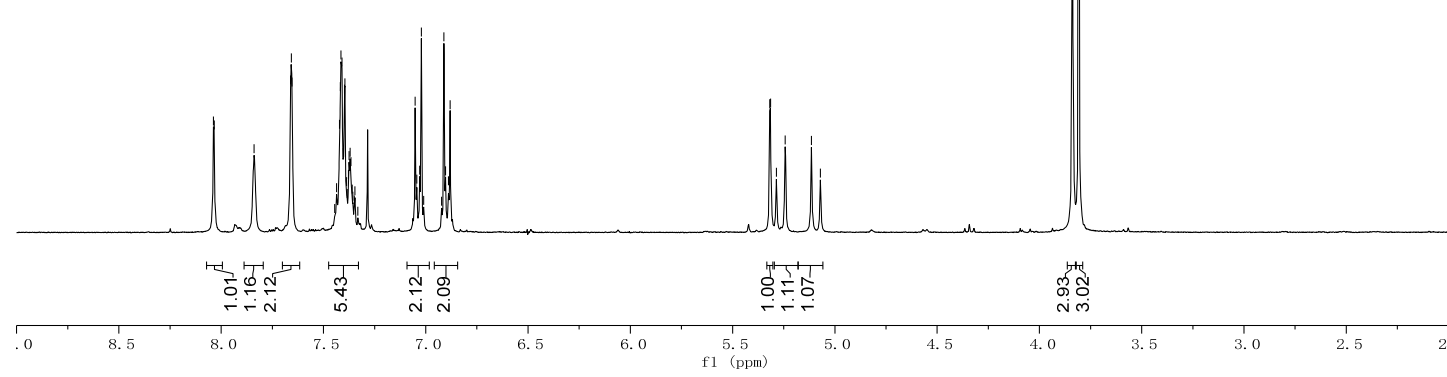

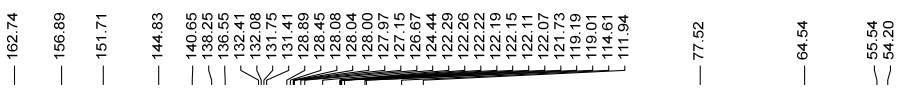

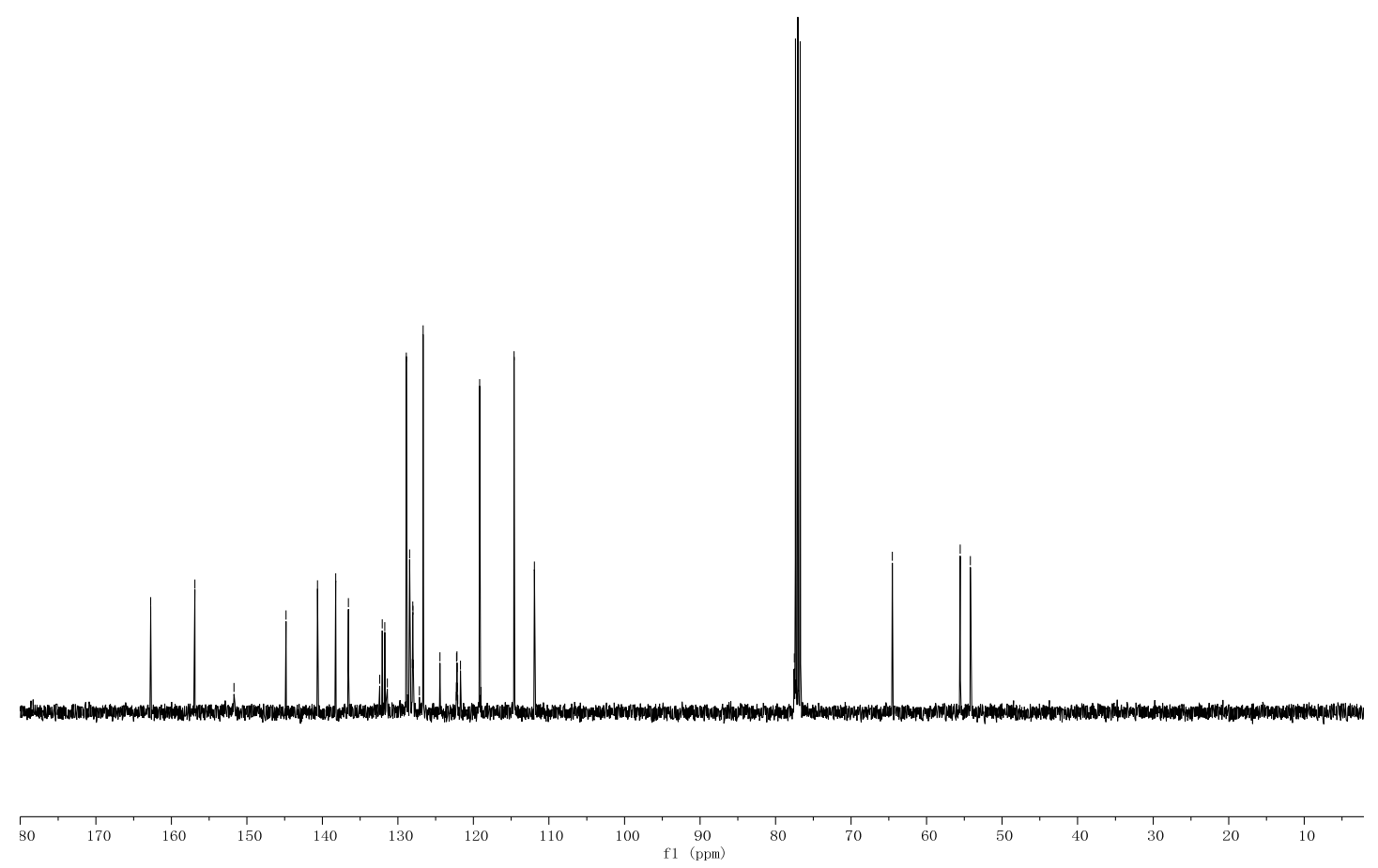


<smiles>CC(=O)N1C=C(C(=O)OCc2cc(C(F)(F)F)cc(C(F)(F)F)c2)C(c2ccccc2)N1c1ccc2ccccc2c1</smiles>

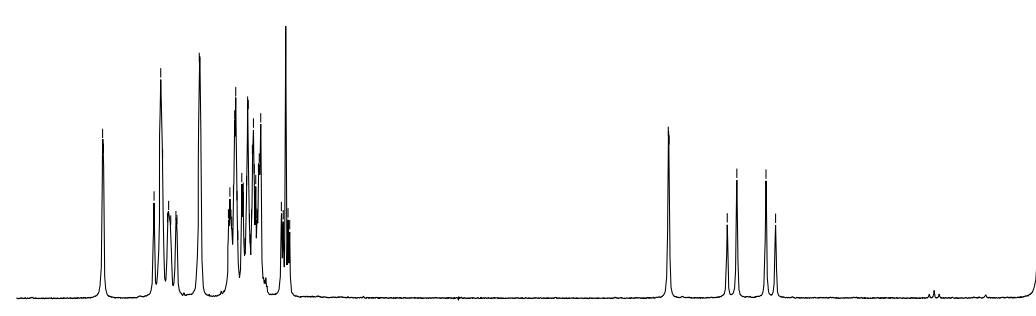

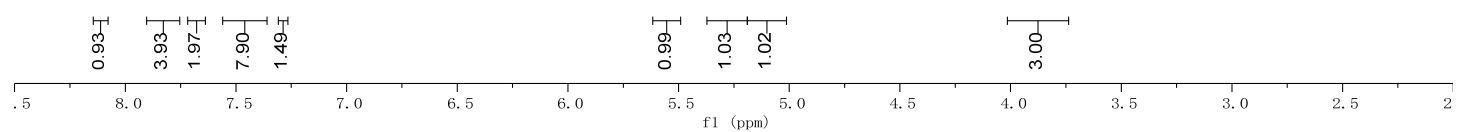

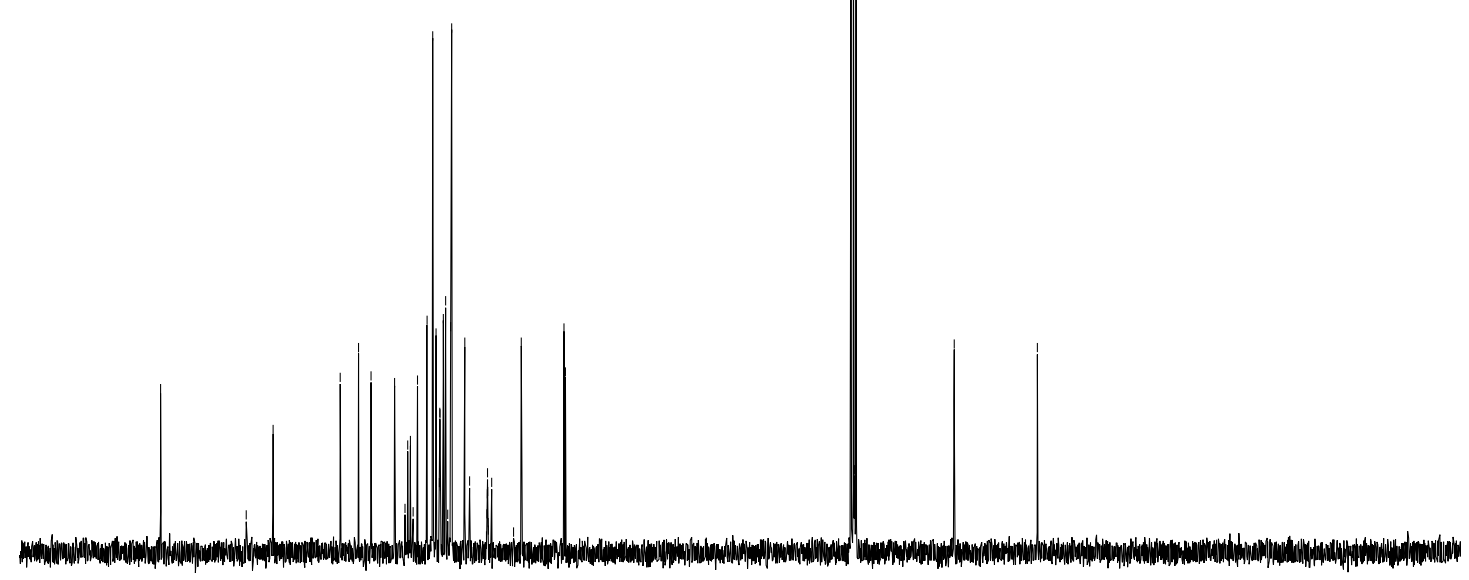

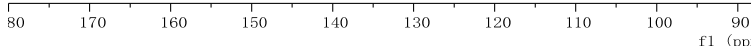




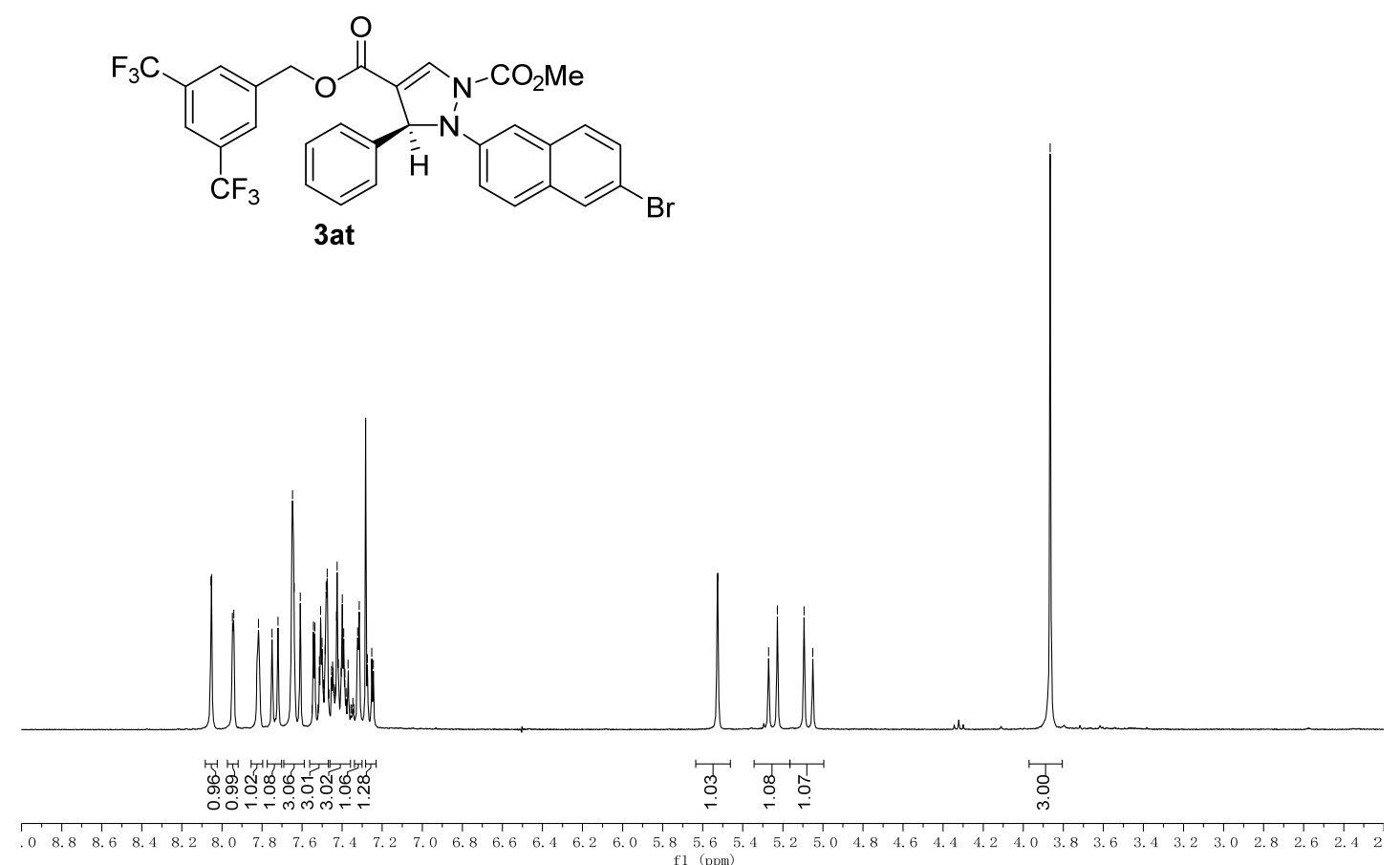

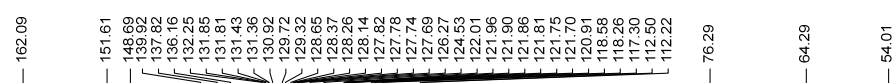

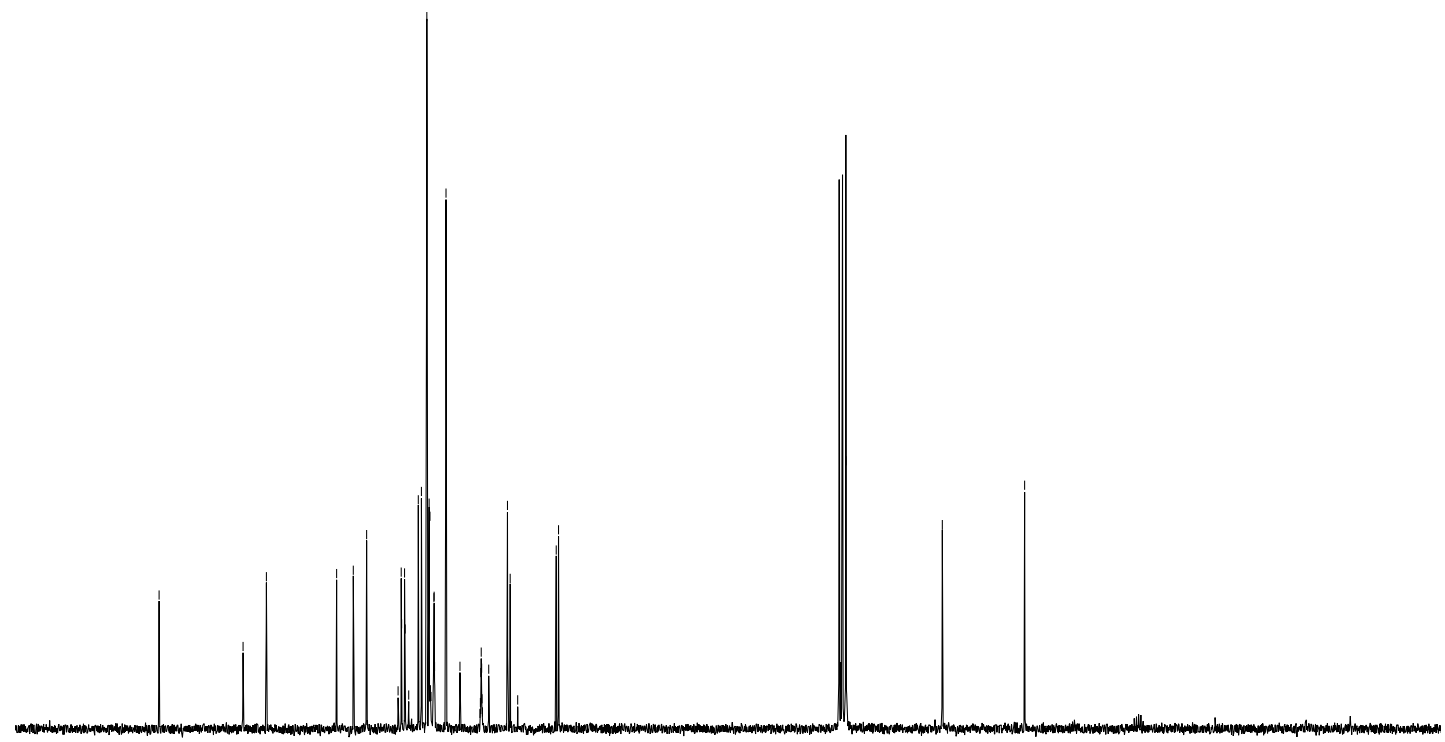

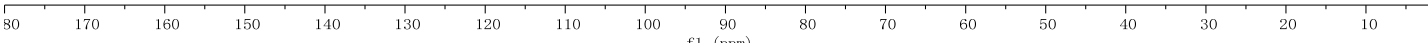




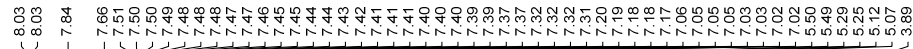<smiles>CC(=O)N1C=C(C(=O)OCc2cc(C(F)(F)F)cc(C(F)(F)F)c2)[C@H](c2ccccc2)[C@H]1c1ccccc1-c1ccc(Cl)cc1</smiles>

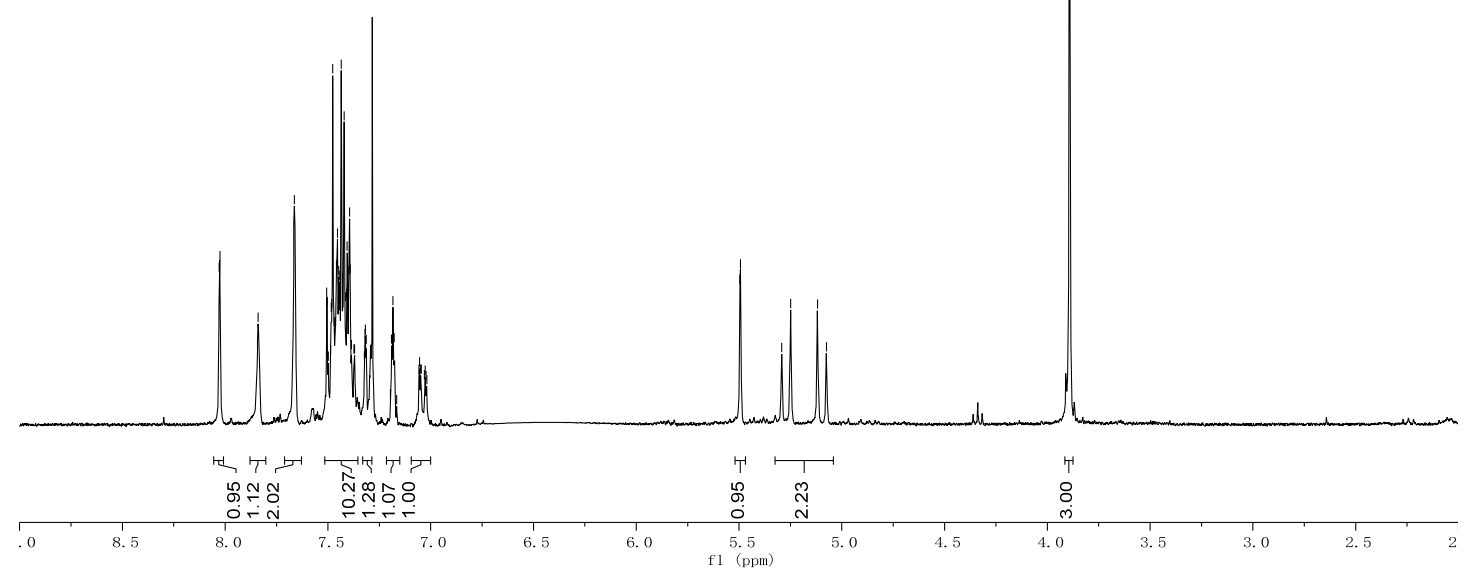

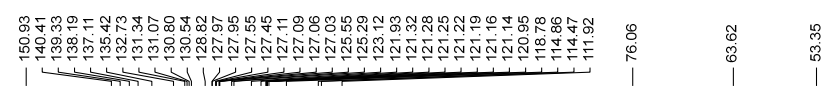

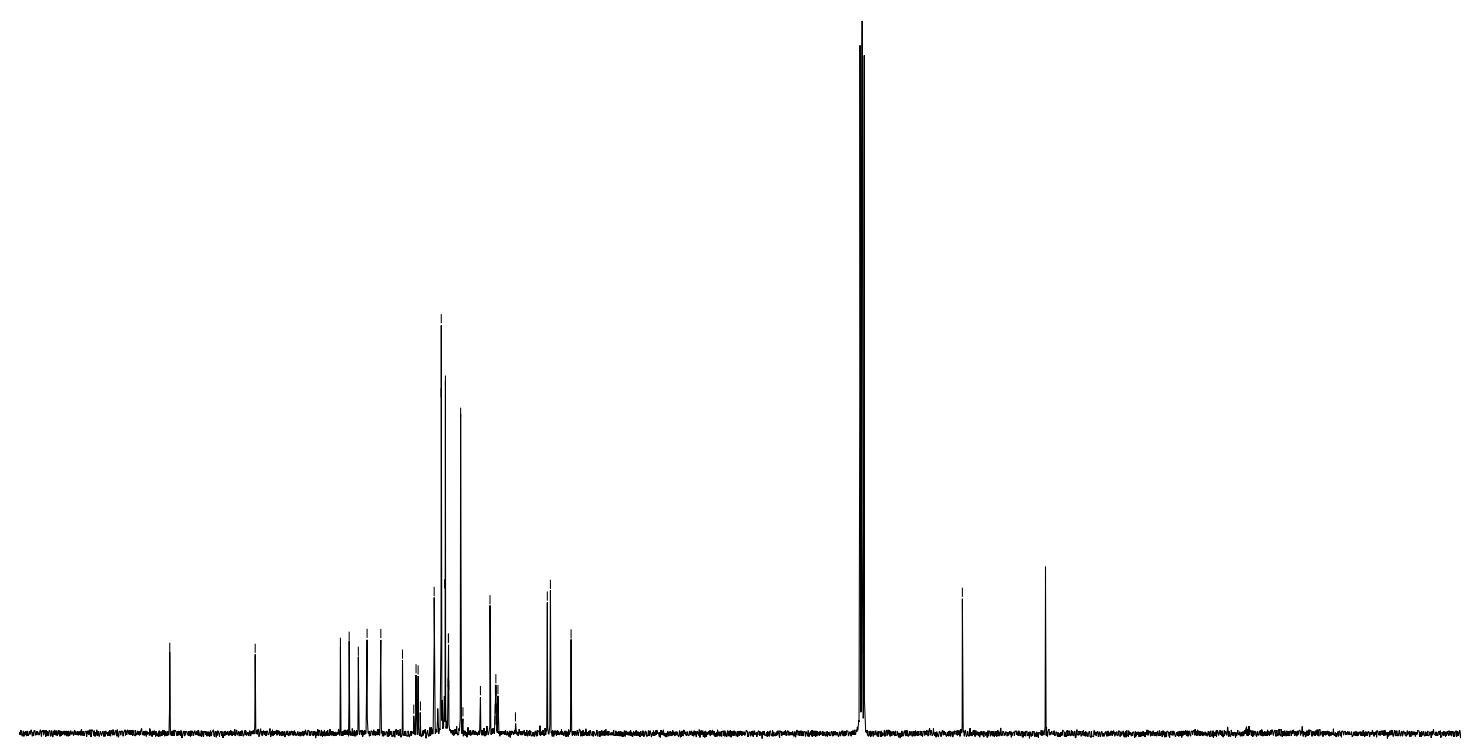

\begin{tabular}{llllllllll}
\hline 80 & 170 & 160 & 150 & 140 & 130 & 120 & 110 & 100 & 90 \\
\hline
\end{tabular} 


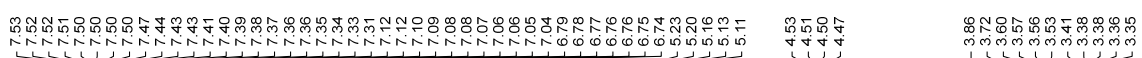

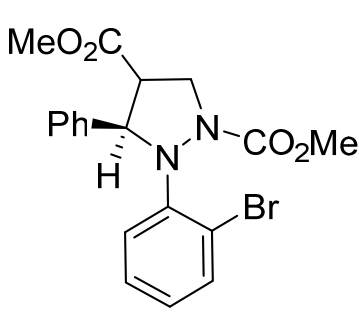

5

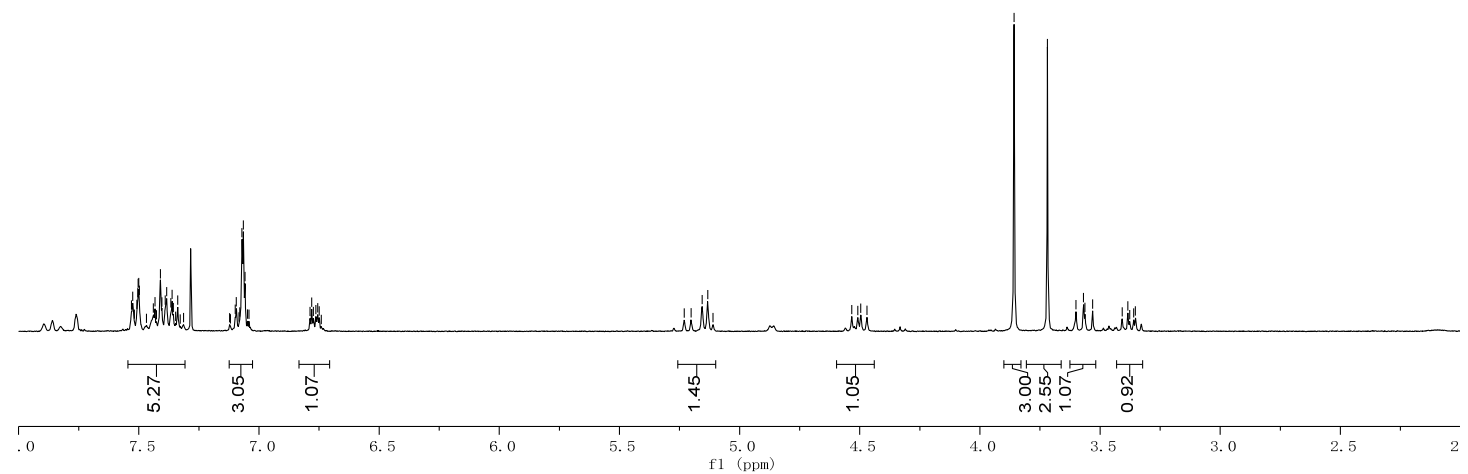

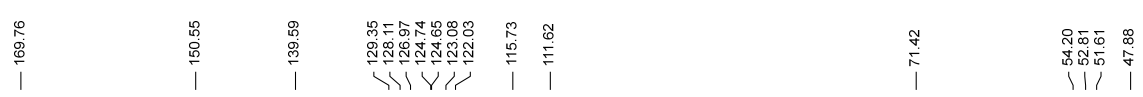

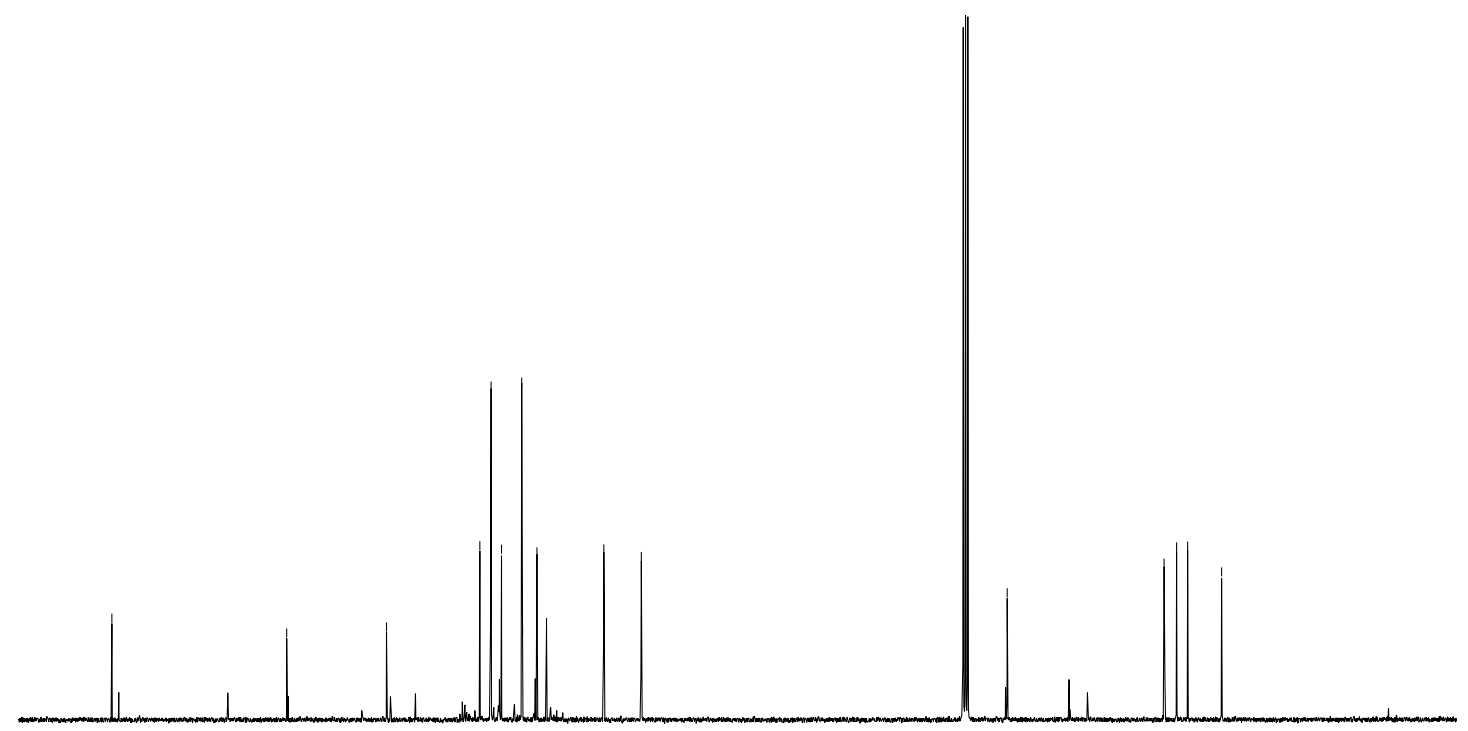

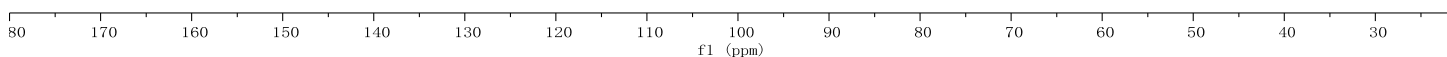


<smiles>CC(=O)N1CC(C(=O)OCc2cc(C(F)(F)F)cc(C(F)(F)F)c2)[C@@H](c2ccccc2)N1c1ccccc1Br</smiles>
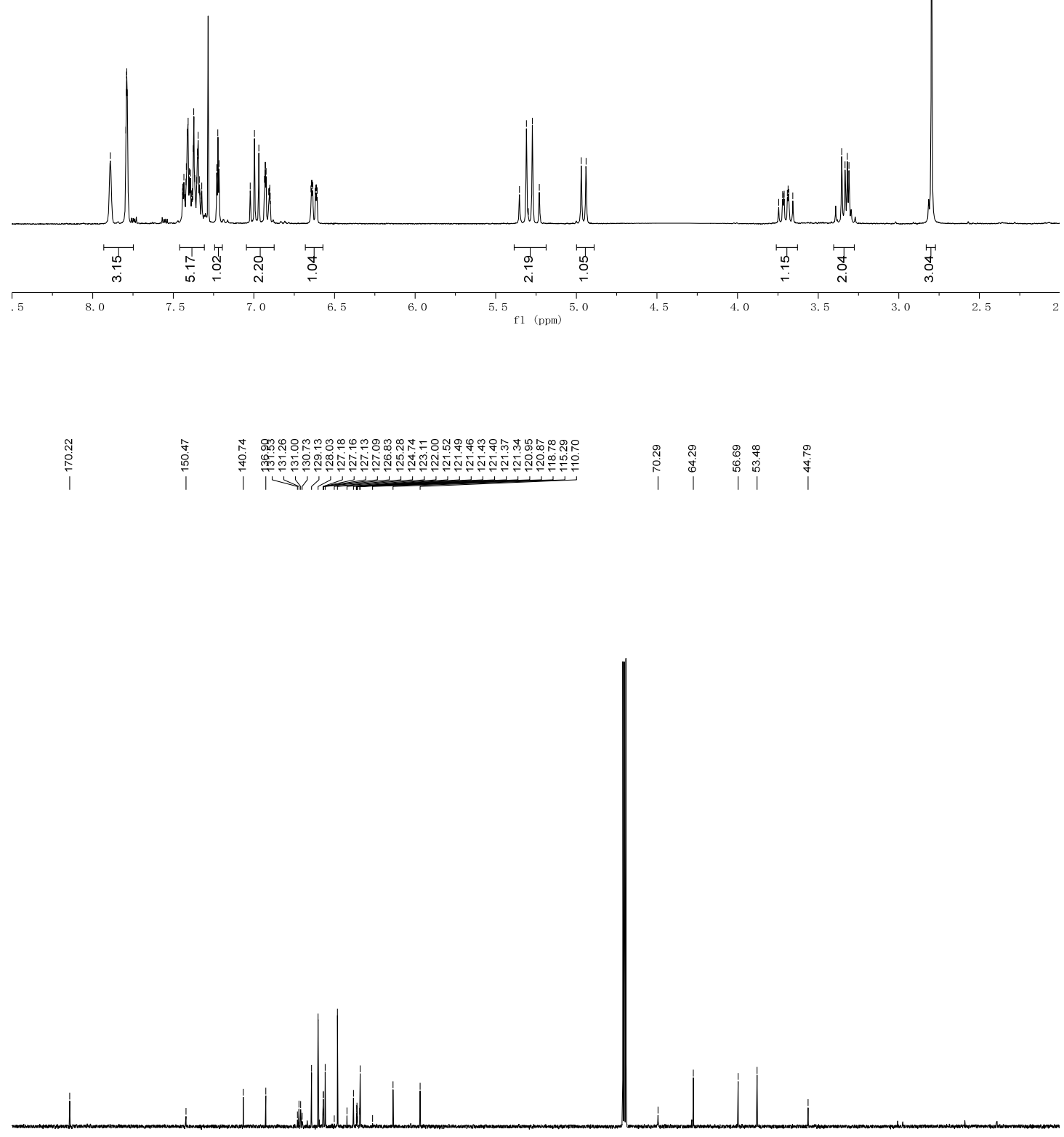

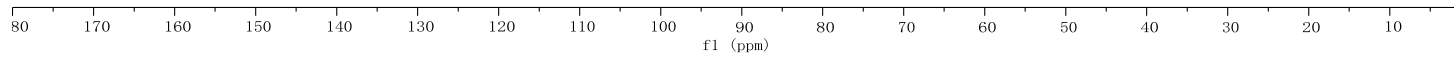




\section{X-Ray Crystallographic Data of 3ha}

Crystallographic data for $\mathbf{3 h a}$ have been deposited with the Cam-bridge Crystallographic Data Centre as deposition number CCDC 1940604. These data can be obtained free of charge via www.ccdc.cam. ac.uk/data request/cif, or by emailing data_request@ccdc.cam.ac.uk, or by contacting The Cambridge Crystallographic Data Centre, 12, Union Road, Cambridge CB2 1EZ, UK; fax: +44 1223336033 .<smiles>CC(=O)N1C=C(C(=O)OCc2cc(C(F)(F)F)cc(C(F)(F)F)c2)C(c2cccc(Br)c2)[C@H]1c1ccccc1</smiles>

Table S1. Crystal Data and Structure Refinement for 3 ha.

Identification code

Empirical formula

Formula weight

Temperature/K

Crystal system

Space group

$\mathrm{a} / \AA ̊$

$\mathrm{b} / \AA$

$\mathrm{c} / \AA ̊$

$\alpha{ }^{\circ}$

$\beta /{ }^{\circ}$

$\gamma^{\circ}$

Volume $/ \AA^{3}$

Z

$\rho_{\text {calc }} \mathrm{g} / \mathrm{cm}^{3}$

$\mu / \mathrm{mm}^{-1}$

$\mathrm{F}(000)$

Crystal size $/ \mathrm{mm}^{3}$ 3ha

$\mathrm{C}_{27} \mathrm{H}_{19} \mathrm{BrF}_{6} \mathrm{~N}_{2} \mathrm{O}_{4}$

629.35

153.15

monoclinic

$\mathrm{P} 2{ }_{1}$

11.661(2)

9.1802(18)

12.632(3)

90

100.26(3)

90

1330.6(5)

2

1.571

1.623

632.0

$0.23 \times 0.15 \times 0.14$ 
Radiation

$2 \Theta$ range for data collection ${ }^{\circ}$

Index ranges

Reflections collected

Independent reflections

Data/restraints/parameters

Goodness-of-fit on $\mathrm{F}^{2}$

Final $R$ indexes $[\mathrm{I}>=2 \sigma(\mathrm{I})]$

Final $\mathrm{R}$ indexes [all data]

Largest diff. peak/hole / e $\AA^{-3}$

Flack parameter
$\operatorname{MoK} \alpha(\lambda=0.71073)$

5.242 to 54.952

$-15 \leq \mathrm{h} \leq 14,-11 \leq \mathrm{k} \leq 11,-16 \leq 1 \leq 15$

14984

$6032\left[\mathrm{R}_{\text {int }}=0.0440, \mathrm{R}_{\text {sigma }}=0.0472\right]$

$6032 / 145 / 418$

1.053

$\mathrm{R}_{1}=0.0417, \mathrm{wR}_{2}=0.0957$

$\mathrm{R}_{1}=0.0447, \mathrm{wR}_{2}=0.0984$

$0.50 /-0.40$

$0.002(6)$

Table S2. Fractional Atomic Coordinates $\left(\times 10^{4}\right)$ and Equivalent Isotropic Displacement Parameters $\left(\AA^{2} \times 10^{3}\right)$ for $3 \mathrm{ha} . \mathrm{U}_{\text {eq }}$ is defined as $1 / 3$ of the trace of the orthogonalised $U_{\mathrm{IJ}}$ tensor.

$\begin{array}{lllll}\text { Atom } \boldsymbol{x} & \boldsymbol{y} & \boldsymbol{z} & \mathbf{U}(\mathbf{e q}) \\ \text { Br1 } & -806.2(4) & -920.4(6) & 7352.3(4) & 39.23(14) \\ \text { F1 } & 931(10) & 5612(10) & 9384(7) & 77(2) \\ \text { F2 } & 574(7) & 7712(9) & 9924(8) & 83(2) \\ \text { F3 } & 1928(7) & 6535(12) & 10795(5) & 71(2) \\ \text { F4 } & 5104(10) & 9794(16) & 10622(7) & 91(3) \\ \text { F5 } & 4971(12) & 11073(14) & 9117(13) & 96(3) \\ \text { F6 } & 5931(7) & 9185(17) & 9387(10) & 82(3) \\ \text { O1 } & 4958(3) & 112(3) & 5921(3) & 32.3(7) \\ \text { O2 } & 5805(3) & 2292(4) & 6402(3) & 38.1(8) \\ \text { O3 } & 1067(3) & 5539(4) & 5846(3) & 34.8(7) \\ \text { O4 } & 2876(3) & 6488(3) & 5912(3) & 31.5(7) \\ \text { N1 } & 2865(3) & 1521(4) & 5265(3) & 25.0(7) \\ \text { C1 } & 2686(4) & 1216(4) & 4122(3) & 27.9(9)\end{array}$




\begin{tabular}{|c|c|c|c|c|}
\hline $\mathrm{C} 2$ & $1704(4)$ & $416(5)$ & $3701(4)$ & $36(1)$ \\
\hline $\mathrm{C} 3$ & $1481(5)$ & $77(6)$ & $2605(4)$ & $47.8(14)$ \\
\hline $\mathrm{C} 4$ & $2216(6)$ & $554(7)$ & 1936(4) & $56.7(17)$ \\
\hline $\mathrm{C} 5$ & $3198(7)$ & 1333(8) & $2370(5)$ & $59.9(17)$ \\
\hline C6 & $3446(6)$ & $1686(7)$ & $3456(4)$ & $45.6(14)$ \\
\hline $\mathrm{C} 7$ & $6892(5)$ & $1549(7)$ & $6788(5)$ & $43.7(13)$ \\
\hline $\mathrm{C} 8$ & $4926(4)$ & $1423(5)$ & $5986(3)$ & $28.5(9)$ \\
\hline $\mathrm{N} 2$ & $3952(3)$ & $2247(4)$ & $5620(3)$ & $27.6(7)$ \\
\hline $\mathrm{C} 10$ & $3773(4)$ & $3710(4)$ & $5780(3)$ & $28.5(9)$ \\
\hline $\mathrm{C} 11$ & $2635(3)$ & $3992(6)$ & $5685(3)$ & $25.2(7)$ \\
\hline $\mathrm{C} 12$ & 1979(4) & $2561(4)$ & $5576(3)$ & $23.4(8)$ \\
\hline C13 & 1599(4) & $2065(4)$ & $6613(3)$ & $25.5(8)$ \\
\hline $\mathrm{C} 14$ & $700(4)$ & $1049(4)$ & $6540(3)$ & $25.0(8)$ \\
\hline $\mathrm{C} 15$ & $383(4)$ & $516(5)$ & $7473(3)$ & $27.5(8)$ \\
\hline $\mathrm{C} 16$ & $922(4)$ & $973(5)$ & $8480(4)$ & $32.3(9)$ \\
\hline $\mathrm{C} 17$ & $1808(4)$ & 1999(6) & $8552(4)$ & $36.5(10)$ \\
\hline $\mathrm{C} 18$ & 2151(4) & $2533(5)$ & $7629(3)$ & $32.2(9)$ \\
\hline C19 & 2093(4) & $5383(5)$ & $5821(3)$ & $25.5(9)$ \\
\hline $\mathrm{C} 20$ & $2466(5)$ & $7901(5)$ & $6207(4)$ & $34.4(10)$ \\
\hline $\mathrm{C} 21$ & 2707(4) & $8041(4)$ & $7420(3)$ & $28.2(9)$ \\
\hline $\mathrm{C} 22$ & 1987(4) & $7361(5)$ & $8043(3)$ & 29.1(9) \\
\hline $\mathrm{C} 23$ & $2237(4)$ & $7475(5)$ & $9156(3)$ & $30.3(9)$ \\
\hline $\mathrm{C} 24$ & $3197(4)$ & $8242(5)$ & $9660(4)$ & $33.5(10)$ \\
\hline $\mathrm{C} 25$ & $3907(4)$ & $8929(6)$ & $9035(4)$ & $35.2(10)$ \\
\hline $\mathrm{C} 26$ & $3663(4)$ & $8825(5)$ & $7925(4)$ & $36.1(11)$ \\
\hline $\mathrm{C} 27$ & $1455(5)$ & $6765(6)$ & $9815(4)$ & $39.4(11)$ \\
\hline $\mathrm{C} 28$ & $4940(5)$ & $9772(7)$ & $9558(5)$ & $54.2(15)$ \\
\hline $\mathrm{F} 2 \mathrm{~A}$ & $1180(14)$ & $7561(17)$ & $10578(12)$ & $79(3)$ \\
\hline F3A & $1998(11)$ & $5615(17)$ & $10398(12)$ & $77(3)$ \\
\hline
\end{tabular}




$\begin{array}{lllll}\text { F1A } & 478(10) & 6270(18) & 9299(10) & 64(3) \\ \text { F5A } & 5371(13) & 10670(20) & 8891(11) & 81(3) \\ \text { F6A } & 5802(12) & 8990(20) & 9988(15) & 103(4) \\ \text { F4A } & 4679(11) & 10714(16) & 10290(11) & 82(3)\end{array}$

Table S3. Anisotropic Displacement Parameters $\left(\AA^{2} \times 10^{3}\right)$ for 3 ha. The Anisotropic displacement factor exponent takes the form: $-2 \pi^{2}\left[h^{2} a * 2 U_{11}+2 h k a * b * U_{12}+\ldots\right]$.

\begin{tabular}{|c|c|c|c|c|c|c|}
\hline Atom & $U_{11}$ & $\mathbf{U}_{22}$ & $\mathbf{U}_{33}$ & $\mathbf{U}_{23}$ & $\mathbf{U}_{13}$ & $\mathbf{U}_{12}$ \\
\hline Br1 & $30.3(2)$ & $34.1(2)$ & $54.3(3)$ & $2.4(2)$ & $10.53(17)$ & $-5.5(2)$ \\
\hline $\mathrm{F} 1$ & $110(6)$ & $69(5)$ & $61(4)$ & $-16(4)$ & $38(4)$ & $-53(4)$ \\
\hline $\mathrm{F} 2$ & $71(4)$ & $93(5)$ & $100(5)$ & $42(4)$ & $56(4)$ & $26(4)$ \\
\hline F3 & $68(4)$ & $111(6)$ & $30(3)$ & $26(3)$ & $2(3)$ & $-27(4)$ \\
\hline $\mathrm{F} 4$ & $78(5)$ & $131(7)$ & $62(5)$ & $-36(5)$ & $6(4)$ & $-53(5)$ \\
\hline F5 & $83(7)$ & $70(6)$ & $127(7)$ & $-15(5)$ & $-5(6)$ & $-32(5)$ \\
\hline F6 & $38(3)$ & $111(6)$ & $96(6)$ & $-34(6)$ & $11(4)$ & $-15(5)$ \\
\hline $\mathrm{O} 1$ & $32.0(16)$ & $27.4(15)$ & $38.9(17)$ & $1.3(13)$ & $9.8(13)$ & $7.2(13)$ \\
\hline $\mathrm{O} 2$ & $29.0(17)$ & $32.2(17)$ & $50(2)$ & $0.3(15)$ & $-0.1(15)$ & $2.1(14)$ \\
\hline $\mathrm{O} 3$ & $31.7(17)$ & $34.9(17)$ & $36.3(16)$ & $-6.7(14)$ & $2.3(13)$ & $6.4(14)$ \\
\hline $\mathrm{O} 4$ & $41.3(18)$ & $18.9(14)$ & $37.4(17)$ & $-2.4(12)$ & $15.3(14)$ & $-0.3(13)$ \\
\hline N1 & $24.6(17)$ & $22.9(17)$ & $27.6(17)$ & $-3.0(14)$ & $5.1(14)$ & $0.8(14)$ \\
\hline $\mathrm{C} 1$ & $37(2)$ & $20.5(19)$ & $26(2)$ & $-2.1(15)$ & $6.8(17)$ & $8.8(17)$ \\
\hline $\mathrm{C} 2$ & $41(3)$ & $34(2)$ & $32(2)$ & $-4.4(18)$ & $2.5(19)$ & $8(2)$ \\
\hline $\mathrm{C} 3$ & $59(3)$ & $40(3)$ & $38(3)$ & $-13(2)$ & $-7(2)$ & $16(3)$ \\
\hline $\mathrm{C} 4$ & $85(5)$ & $55(3)$ & $30(2)$ & $-7(2)$ & $10(3)$ & $24(3)$ \\
\hline $\mathrm{C} 5$ & $83(5)$ & $65(4)$ & $38(3)$ & $0(3)$ & $29(3)$ & $9(4)$ \\
\hline $\mathrm{C} 6$ & $57(4)$ & $45(3)$ & $40(3)$ & $1(2)$ & $23(3)$ & $1(3)$ \\
\hline $\mathrm{C} 7$ & $29(3)$ & $49(3)$ & $50(3)$ & $4(3)$ & $0(2)$ & $8(2)$ \\
\hline $\mathrm{C} 8$ & $29(2)$ & $30(2)$ & $27(2)$ & $1.2(17)$ & $8.6(16)$ & $4.5(17)$ \\
\hline
\end{tabular}




\begin{tabular}{|c|c|c|c|c|c|c|}
\hline $\mathrm{N} 2$ & $24.8(17)$ & $22.4(17)$ & $35.3(18)$ & $-4.2(14)$ & $4.9(14)$ & $0.5(14)$ \\
\hline $\mathrm{C} 10$ & $34(2)$ & $24(2)$ & $28(2)$ & $-0.9(14)$ & $8.9(17)$ & $-0.1(15)$ \\
\hline $\mathrm{C} 11$ & $32.1(18)$ & $21.7(17)$ & $22.3(16)$ & $1.3(18)$ & $6.5(13)$ & $4(2)$ \\
\hline $\mathrm{C} 12$ & $29(2)$ & 19.1(19) & $22.4(19)$ & $-0.1(15)$ & $4.8(16)$ & $1.8(17)$ \\
\hline C13 & $27(2)$ & 23.3(19) & $26(2)$ & $-2.9(16)$ & $5.9(16)$ & $2.1(15)$ \\
\hline $\mathrm{C} 14$ & $23.3(19)$ & $24(2)$ & $28(2)$ & $-2.8(15)$ & $4.9(16)$ & $3.1(15)$ \\
\hline $\mathrm{C} 15$ & $25(2)$ & $25(2)$ & $34(2)$ & $1.4(16)$ & $8.0(16)$ & $4.1(16)$ \\
\hline $\mathrm{C} 16$ & $35(2)$ & $32(2)$ & $32(2)$ & $-1.9(18)$ & $11.6(18)$ & $0.3(19)$ \\
\hline $\mathrm{C} 17$ & $43(3)$ & $43(3)$ & $23(2)$ & $-5.2(18)$ & $3.2(18)$ & $-6(2)$ \\
\hline $\mathrm{C} 18$ & $34(2)$ & $36(2)$ & $25(2)$ & $-4.5(17)$ & $1.6(17)$ & $-5.8(19)$ \\
\hline C19 & $32(2)$ & $23(2)$ & $20.7(19)$ & $3.3(15)$ & $2.0(17)$ & $-0.6(17)$ \\
\hline $\mathrm{C} 20$ & $52(3)$ & $18.2(19)$ & $35(2)$ & $2.0(17)$ & $13(2)$ & $5.4(19)$ \\
\hline $\mathrm{C} 21$ & $38(2)$ & $17.6(17)$ & $30(2)$ & $0.4(16)$ & $9.6(18)$ & $4.6(17)$ \\
\hline $\mathrm{C} 22$ & $33(2)$ & $25(2)$ & $28(2)$ & $1.5(16)$ & $2.4(17)$ & $2.3(17)$ \\
\hline $\mathrm{C} 23$ & $29(2)$ & $31(2)$ & $31(2)$ & $0.2(17)$ & $5.4(17)$ & $2.4(18)$ \\
\hline $\mathrm{C} 24$ & $37(2)$ & $31(2)$ & $33(2)$ & $-3.9(17)$ & $4.0(19)$ & $3.6(19)$ \\
\hline $\mathrm{C} 25$ & $33(2)$ & $32(2)$ & $41(2)$ & $-7(2)$ & $7.1(17)$ & $-4(2)$ \\
\hline $\mathrm{C} 26$ & $44(2)$ & $28(3)$ & $39(2)$ & $-1.5(18)$ & $17.3(19)$ & $-0.5(19)$ \\
\hline $\mathrm{C} 27$ & $41(3)$ & $53(3)$ & $25(2)$ & $2(2)$ & $5.8(19)$ & $-5(2)$ \\
\hline $\mathrm{C} 28$ & $46(3)$ & $60(4)$ & $57(4)$ & $-8(3)$ & $9(3)$ & $-13(3)$ \\
\hline $\mathrm{F} 2 \mathrm{~A}$ & $87(7)$ & $93(6)$ & $71(6)$ & $-24(5)$ & $48(5)$ & $-21(6)$ \\
\hline F3A & $66(5)$ & $89(7)$ & $83(6)$ & $40(5)$ & $29(5)$ & $-5(6)$ \\
\hline F1A & $46(5)$ & $100(8)$ & $45(4)$ & $11(6)$ & $4(4)$ & $-35(5)$ \\
\hline F5A & $72(6)$ & $98(7)$ & $79(5)$ & $-15(5)$ & $29(5)$ & $-50(5)$ \\
\hline F6A & $60(5)$ & $110(6)$ & $122(8)$ & $-4(7)$ & $-31(6)$ & $-13(6)$ \\
\hline $\mathrm{F} 4 \mathrm{~A}$ & $69(6)$ & $91(6)$ & $89(6)$ & $-59(5)$ & $24(5)$ & $-42(5)$ \\
\hline
\end{tabular}


Table S4. Bond Lengths for 3ha.

\begin{tabular}{|c|c|c|c|c|c|}
\hline Atom & Atom & Length/Å & Aton & Atom & Length/Å \\
\hline $\mathrm{Br} 1$ & $\mathrm{C} 15$ & $1.900(4)$ & $\mathrm{C} 11$ & $\mathrm{C} 12$ & $1.514(6)$ \\
\hline $\mathrm{F} 1$ & $\mathrm{C} 27$ & $1.293(9)$ & $\mathrm{C} 11$ & C19 & $1.449(7)$ \\
\hline $\mathrm{F} 2$ & $\mathrm{C} 27$ & $1.371(9)$ & $\mathrm{C} 12$ & $\mathrm{C} 13$ & $1.525(6)$ \\
\hline F3 & $\mathrm{C} 27$ & $1.280(7)$ & $\mathrm{C} 13$ & $\mathrm{C} 14$ & $1.393(6)$ \\
\hline $\mathrm{F} 4$ & $\mathrm{C} 28$ & $1.323(10)$ & $\mathrm{C} 13$ & $\mathrm{C} 18$ & $1.397(6)$ \\
\hline F5 & $\mathrm{C} 28$ & $1.321(15)$ & $\mathrm{C} 14$ & $\mathrm{C} 15$ & $1.386(6)$ \\
\hline F6 & $\mathrm{C} 28$ & $1.327(12)$ & $\mathrm{C} 15$ & $\mathrm{C} 16$ & $1.379(6)$ \\
\hline $\mathrm{O} 1$ & $\mathrm{C} 8$ & $1.208(5)$ & $\mathrm{C} 16$ & $\mathrm{C} 17$ & $1.389(7)$ \\
\hline $\mathrm{O} 2$ & $\mathrm{C} 7$ & $1.446(6)$ & $\mathrm{C} 17$ & $\mathrm{C} 18$ & $1.388(6)$ \\
\hline $\mathrm{O} 2$ & $\mathrm{C} 8$ & $1.330(5)$ & $\mathrm{C} 20$ & $\mathrm{C} 21$ & $1.513(6)$ \\
\hline $\mathrm{O} 3$ & $\mathrm{C} 19$ & $1.210(6)$ & $\mathrm{C} 21$ & $\mathrm{C} 22$ & $1.396(6)$ \\
\hline $\mathrm{O} 4$ & $\mathrm{C} 19$ & $1.356(5)$ & $\mathrm{C} 21$ & $\mathrm{C} 26$ & $1.385(6)$ \\
\hline $\mathrm{O} 4$ & $\mathrm{C} 20$ & $1.453(5)$ & $\mathrm{C} 22$ & $\mathrm{C} 23$ & $1.388(6)$ \\
\hline N1 & $\mathrm{C} 1$ & $1.449(5)$ & $\mathrm{C} 23$ & $\mathrm{C} 24$ & $1.378(6)$ \\
\hline N1 & N2 & $1.431(5)$ & $\mathrm{C} 23$ & $\mathrm{C} 27$ & $1.490(6)$ \\
\hline N1 & $\mathrm{C} 12$ & $1.509(5)$ & $\mathrm{C} 24$ & $\mathrm{C} 25$ & $1.392(7)$ \\
\hline $\mathrm{C} 1$ & $\mathrm{C} 2$ & $1.384(7)$ & $\mathrm{C} 25$ & $\mathrm{C} 26$ & $1.383(6)$ \\
\hline $\mathrm{C} 1$ & $\mathrm{C} 6$ & $1.394(7)$ & $\mathrm{C} 25$ & $\mathrm{C} 28$ & $1.485(7)$ \\
\hline $\mathrm{C} 2$ & $\mathrm{C} 3$ & $1.398(7)$ & $\mathrm{C} 27$ & $\mathrm{~F} 2 \mathrm{~A}$ & $1.294(12)$ \\
\hline $\mathrm{C} 3$ & $\mathrm{C} 4$ & $1.379(9)$ & $\mathrm{C} 27$ & F3A & $1.375(13)$ \\
\hline $\mathrm{C} 4$ & $\mathrm{C} 5$ & $1.378(10)$ & $\mathrm{C} 27$ & F1A & $1.290(12)$ \\
\hline $\mathrm{C} 5$ & $\mathrm{C} 6$ & $1.389(8)$ & $\mathrm{C} 28$ & F5A & $1.338(15)$ \\
\hline $\mathrm{C} 8$ & $\mathrm{~N} 2$ & $1.374(5)$ & $\mathrm{C} 28$ & F6A & $1.275(16)$ \\
\hline N2 & $\mathrm{C} 10$ & $1.380(5)$ & $\mathrm{C} 28$ & $\mathrm{~F} 4 \mathrm{~A}$ & $1.340(12)$ \\
\hline $\mathrm{C} 10$ & $\mathrm{C} 11$ & $1.336(6)$ & & & \\
\hline
\end{tabular}


Table S5. Bond Angles for $3 \mathrm{ha}$.

\begin{tabular}{|c|c|c|c|c|c|c|c|}
\hline Atom & Atom & Atom & Angle $/^{\circ}$ & Atom & Atom & Atom & Angle $/^{\circ}$ \\
\hline $\mathrm{C} 8$ & $\mathrm{O} 2$ & $\mathrm{C} 7$ & $114.7(4)$ & $\mathrm{O} 4$ & $\mathrm{C} 19$ & $\mathrm{C} 11$ & $111.6(4)$ \\
\hline C19 & $\mathrm{O} 4$ & $\mathrm{C} 20$ & $116.2(4)$ & $\mathrm{O} 4$ & $\mathrm{C} 20$ & $\mathrm{C} 21$ & 109.0(3) \\
\hline $\mathrm{C} 1$ & N1 & $\mathrm{C} 12$ & $113.4(3)$ & $\mathrm{C} 22$ & $\mathrm{C} 21$ & $\mathrm{C} 20$ & $120.7(4)$ \\
\hline N2 & N1 & $\mathrm{C} 1$ & $111.4(3)$ & $\mathrm{C} 26$ & $\mathrm{C} 21$ & $\mathrm{C} 20$ & $120.0(4)$ \\
\hline N2 & N1 & $\mathrm{C} 12$ & $103.5(3)$ & $\mathrm{C} 26$ & $\mathrm{C} 21$ & $\mathrm{C} 22$ & $119.3(4)$ \\
\hline $\mathrm{C} 2$ & $\mathrm{C} 1$ & N1 & $116.6(4)$ & $\mathrm{C} 23$ & $\mathrm{C} 22$ & $\mathrm{C} 21$ & $119.9(4)$ \\
\hline $\mathrm{C} 2$ & $\mathrm{C} 1$ & $\mathrm{C} 6$ & $120.2(4)$ & $\mathrm{C} 22$ & $\mathrm{C} 23$ & $\mathrm{C} 27$ & $119.6(4)$ \\
\hline C6 & $\mathrm{C} 1$ & N1 & $123.1(4)$ & $\mathrm{C} 24$ & $\mathrm{C} 23$ & $\mathrm{C} 22$ & $120.9(4)$ \\
\hline $\mathrm{C} 1$ & $\mathrm{C} 2$ & $\mathrm{C} 3$ & $119.6(5)$ & $\mathrm{C} 24$ & $\mathrm{C} 23$ & $\mathrm{C} 27$ & $119.5(4)$ \\
\hline $\mathrm{C} 4$ & $\mathrm{C} 3$ & $\mathrm{C} 2$ & $120.7(6)$ & $\mathrm{C} 23$ & $\mathrm{C} 24$ & $\mathrm{C} 25$ & $119.0(4)$ \\
\hline $\mathrm{C} 5$ & $\mathrm{C} 4$ & $\mathrm{C} 3$ & $119.0(5)$ & $\mathrm{C} 24$ & $\mathrm{C} 25$ & $\mathrm{C} 28$ & $120.1(4)$ \\
\hline $\mathrm{C} 4$ & $\mathrm{C} 5$ & C6 & $121.8(6)$ & $\mathrm{C} 26$ & $\mathrm{C} 25$ & $\mathrm{C} 24$ & $120.5(4)$ \\
\hline $\mathrm{C} 5$ & C6 & $\mathrm{C} 1$ & $118.7(6)$ & $\mathrm{C} 26$ & $\mathrm{C} 25$ & $\mathrm{C} 28$ & $119.4(5)$ \\
\hline $\mathrm{O} 1$ & $\mathrm{C} 8$ & $\mathrm{O} 2$ & $126.4(4)$ & $\mathrm{C} 25$ & $\mathrm{C} 26$ & $\mathrm{C} 21$ & $120.4(4)$ \\
\hline $\mathrm{O} 1$ & $\mathrm{C} 8$ & $\mathrm{~N} 2$ & $124.0(4)$ & $\mathrm{F} 1$ & $\mathrm{C} 27$ & $\mathrm{~F} 2$ & $104.8(7)$ \\
\hline $\mathrm{O} 2$ & $\mathrm{C} 8$ & $\mathrm{~N} 2$ & $109.6(4)$ & $\mathrm{F} 1$ & $\mathrm{C} 27$ & $\mathrm{C} 23$ & $114.4(5)$ \\
\hline C8 & N2 & N1 & $118.8(3)$ & $\mathrm{F} 2$ & $\mathrm{C} 27$ & $\mathrm{C} 23$ & $108.8(5)$ \\
\hline C8 & N2 & $\mathrm{C} 10$ & $128.6(4)$ & F3 & $\mathrm{C} 27$ & $\mathrm{~F} 1$ & $111.2(7)$ \\
\hline $\mathrm{C} 10$ & N2 & N1 & $110.5(3)$ & F3 & $\mathrm{C} 27$ & $\mathrm{~F} 2$ & 102.1(7) \\
\hline C11 & $\mathrm{C} 10$ & $\mathrm{~N} 2$ & $110.4(4)$ & F3 & $\mathrm{C} 27$ & $\mathrm{C} 23$ & $114.3(5)$ \\
\hline $\mathrm{C} 10$ & $\mathrm{C} 11$ & $\mathrm{C} 12$ & $108.5(4)$ & $\mathrm{F} 2 \mathrm{~A}$ & $\mathrm{C} 27$ & $\mathrm{C} 23$ & $115.3(7)$ \\
\hline C10 & C11 & C19 & $127.4(4)$ & $\mathrm{F} 2 \mathrm{~A}$ & $\mathrm{C} 27$ & F3A & $100.7(10)$ \\
\hline C19 & $\mathrm{C} 11$ & $\mathrm{C} 12$ & $123.5(3)$ & F3A & $\mathrm{C} 27$ & $\mathrm{C} 23$ & $111.5(6)$ \\
\hline N1 & $\mathrm{C} 12$ & $\mathrm{C} 11$ & 102.4(3) & F1A & $\mathrm{C} 27$ & $\mathrm{C} 23$ & $116.4(7)$ \\
\hline N1 & $\mathrm{C} 12$ & $\mathrm{C} 13$ & $110.6(3)$ & F1A & $\mathrm{C} 27$ & $\mathrm{~F} 2 \mathrm{~A}$ & $105.2(10)$ \\
\hline C11 & $\mathrm{C} 12$ & $\mathrm{C} 13$ & $113.6(3)$ & F1A & $\mathrm{C} 27$ & F3A & $106.3(10)$ \\
\hline $\mathrm{C} 14$ & $\mathrm{C} 13$ & $\mathrm{C} 12$ & $118.2(4)$ & F4 & $\mathrm{C} 28$ & F6 & $101.5(8)$ \\
\hline
\end{tabular}




$\begin{array}{lllllllll}\mathrm{C} 14 & \mathrm{C} 13 & \mathrm{C} 18 & 119.0(4) & \mathrm{F} 4 & \mathrm{C} 28 & \mathrm{C} 25 & 114.8(6) \\ \mathrm{C} 18 & \mathrm{C} 13 & \mathrm{C} 12 & 122.7(4) & \mathrm{F} 5 & \mathrm{C} 28 & \mathrm{~F} 4 & 113.9(9) \\ \mathrm{C} 15 & \mathrm{C} 14 & \mathrm{C} 13 & 119.5(4) & \mathrm{F} 5 & \mathrm{C} 28 & \mathrm{~F} 6 & 102.0(9) \\ \mathrm{C} 14 & \mathrm{C} 15 & \mathrm{Br} 1 & 118.6(3) & \mathrm{F} 5 & \mathrm{C} 28 & \mathrm{C} 25 & 111.4(7) \\ \mathrm{C} 16 & \mathrm{C} 15 & \mathrm{~B} 1 & 119.4(3) & \mathrm{F} 6 & \mathrm{C} 28 & \mathrm{C} 25 & 112.1(7) \\ \mathrm{C} 16 & \mathrm{C} 15 & \mathrm{C} 14 & 122.0(4) & \mathrm{F} 5 \mathrm{~A} & \mathrm{C} 28 & \mathrm{C} 25 & 114.0(8) \\ \mathrm{C} 15 & \mathrm{C} 16 & \mathrm{C} 17 & 118.6(4) & \mathrm{F} 5 \mathrm{~A} & \mathrm{C} 28 & \mathrm{~F} 4 \mathrm{~A} & 101.2(9) \\ \mathrm{C} 18 & \mathrm{C} 17 & \mathrm{C} 16 & 120.5(4) & \mathrm{F} 6 \mathrm{~A} & \mathrm{C} 28 & \mathrm{C} 25 & 114.3(9) \\ \mathrm{C} 17 & \mathrm{C} 18 & \mathrm{C} 13 & 120.5(4) & \mathrm{F} 6 \mathrm{~A} & \mathrm{C} 28 & \mathrm{~F} 5 \mathrm{~A} & 105.0(11) \\ \mathrm{O} 3 & \mathrm{C} 19 & \mathrm{O} 4 & 124.3(4) & \mathrm{F} 6 \mathrm{~A} & \mathrm{C} 28 & \mathrm{~F} 4 \mathrm{~A} & 109.2(10) \\ \mathrm{O} 3 & \mathrm{C} 19 & \mathrm{C} 11 & 124.1(4) & \mathrm{F} 4 \mathrm{~A} & \mathrm{C} 28 & \mathrm{C} 25 & 112.2(6)\end{array}$

Table S6. Torsion Angles for 3 ha.

$\begin{array}{llllllllll}\mathbf{A} & \mathbf{B} & \mathbf{C} & \mathbf{D} & \mathbf{A n g l e} /^{\circ} & \mathbf{A} & \mathbf{B} & \mathbf{C} & \mathbf{D} & \text { Angle }^{\circ} \\ \mathrm{B} 1 & \mathrm{C} 15 & \mathrm{C} 16 & \mathrm{C} 17 & 178.5(4) & \mathrm{C} 14 & \mathrm{C} 13 & \mathrm{C} 18 & \mathrm{C} 17 & -0.2(7) \\ \mathrm{O} 1 & \mathrm{C} 8 & \mathrm{~N} 2 & \mathrm{~N} 1 & 8.5(6) & \mathrm{C} 14 & \mathrm{C} 15 & \mathrm{C} 16 & \mathrm{C} 17 & 0.0(7) \\ \mathrm{O} 1 & \mathrm{C} 8 & \mathrm{~N} 2 & \mathrm{C} 10 & 170.4(4) & \mathrm{C} 15 & \mathrm{C} 16 & \mathrm{C} 17 & \mathrm{C} 18 & -0.9(7) \\ \mathrm{O} 2 & \mathrm{C} 8 & \mathrm{~N} 2 & \mathrm{~N} 1 & -172.2(3) & \mathrm{C} 16 & \mathrm{C} 17 & \mathrm{C} 18 & \mathrm{C} 13 & 1.0(7) \\ \mathrm{O} 2 & \mathrm{C} 8 & \mathrm{~N} 2 & \mathrm{C} 10 & -10.3(6) & \mathrm{C} 18 & \mathrm{C} 13 & \mathrm{C} 14 & \mathrm{C} 15 & -0.6(6) \\ \mathrm{O} 4 & \mathrm{C} 20 & \mathrm{C} 21 & \mathrm{C} 22 & -78.5(5) & \mathrm{C} 19 & \mathrm{O} 4 & \mathrm{C} 20 & \mathrm{C} 21 & 90.4(5) \\ \mathrm{O} 4 & \mathrm{C} 20 & \mathrm{C} 21 & \mathrm{C} 26 & 100.2(5) & \mathrm{C} 19 & \mathrm{C} 11 & \mathrm{C} 12 & \mathrm{~N} 1 & 170.5(3) \\ \mathrm{N} 1 & \mathrm{C} 1 & \mathrm{C} 2 & \mathrm{C} 3 & -179.7(4) & \mathrm{C} 19 & \mathrm{C} 11 & \mathrm{C} 12 & \mathrm{C} 13 & -70.2(5) \\ \mathrm{N} 1 & \mathrm{C} 1 & \mathrm{C} 6 & \mathrm{C} 5 & 179.8(5) & \mathrm{C} 20 & \mathrm{O} 4 & \mathrm{C} 19 & \mathrm{O} 3 & 8.9(6) \\ \mathrm{N} 1 & \mathrm{~N} 2 & \mathrm{C} 10 & \mathrm{C} 11 & 7.6(5) & \mathrm{C} 20 & \mathrm{O} 4 & \mathrm{C} 19 & \mathrm{C} 11 & -171.4(3) \\ \mathrm{N} 1 & \mathrm{C} 12 & \mathrm{C} 13 & \mathrm{C} 14 & -85.5(5) & \mathrm{C} 20 & \mathrm{C} 21 & \mathrm{C} 22 & \mathrm{C} 23 & 178.5(4) \\ \mathrm{N} 1 & \mathrm{C} 12 & \mathrm{C} 13 & \mathrm{C} 18 & 91.3(5) & \mathrm{C} 20 & \mathrm{C} 21 & \mathrm{C} 26 & \mathrm{C} 25 & -178.4(4) \\ \mathrm{C} 1 & \mathrm{~N} 1 & \mathrm{~N} 2 & \mathrm{C} 8 & -91.2(4) & \mathrm{C} 21 & \mathrm{C} 22 & \mathrm{C} 23 & \mathrm{C} 24 & -0.6(7) \\ \mathrm{C} 1 & \mathrm{~N} 1 & \mathrm{~N} 2 & \mathrm{C} 10 & 103.8(4) & \mathrm{C} 21 & \mathrm{C} 22 & \mathrm{C} 23 & \mathrm{C} 27 & 179.1(4)\end{array}$




\begin{tabular}{|c|c|c|c|c|c|c|c|c|c|}
\hline $\mathrm{C} 1$ & $\mathrm{~N} 1$ & $\mathrm{C} 12$ & $\mathrm{C} 11$ & $-99.9(4)$ & $\mathrm{C} 22$ & $\mathrm{C} 21$ & $\mathrm{C} 26$ & $\mathrm{C} 25$ & $0.3(7)$ \\
\hline $\mathrm{C} 1$ & N1 & $\mathrm{C} 12$ & $\mathrm{C} 13$ & $138.8(4)$ & $\mathrm{C} 22$ & $\mathrm{C} 23$ & $\mathrm{C} 24$ & $\mathrm{C} 25$ & $1.2(7)$ \\
\hline $\mathrm{C} 1$ & $\mathrm{C} 2$ & $\mathrm{C} 3$ & $\mathrm{C} 4$ & $-1.2(7)$ & $\mathrm{C} 22$ & $\mathrm{C} 23$ & $\mathrm{C} 27$ & $\mathrm{~F} 1$ & $29.3(9)$ \\
\hline $\mathrm{C} 2$ & $\mathrm{C} 1$ & C6 & $\mathrm{C} 5$ & $0.0(8)$ & $\mathrm{C} 22$ & $\mathrm{C} 23$ & $\mathrm{C} 27$ & $\mathrm{~F} 2$ & $-87.4(7)$ \\
\hline $\mathrm{C} 2$ & $\mathrm{C} 3$ & $\mathrm{C} 4$ & $\mathrm{C} 5$ & $2.0(8)$ & $\mathrm{C} 22$ & $\mathrm{C} 23$ & $\mathrm{C} 27$ & F3 & $159.2(7)$ \\
\hline $\mathrm{C} 3$ & $\mathrm{C} 4$ & $\mathrm{C} 5$ & $\mathrm{C} 6$ & $-1.9(9)$ & $\mathrm{C} 22$ & $\mathrm{C} 23$ & $\mathrm{C} 27$ & $\mathrm{~F} 2 \mathrm{~A}$ & $-134.4(11)$ \\
\hline $\mathrm{C} 4$ & $\mathrm{C} 5$ & C6 & $\mathrm{C} 1$ & $0.9(9)$ & $\mathrm{C} 22$ & $\mathrm{C} 23$ & $\mathrm{C} 27$ & F3A & $111.6(9)$ \\
\hline C6 & $\mathrm{C} 1$ & $\mathrm{C} 2$ & $\mathrm{C} 3$ & $0.2(7)$ & $\mathrm{C} 22$ & $\mathrm{C} 23$ & $\mathrm{C} 27$ & F1A & $-10.5(11)$ \\
\hline $\mathrm{C} 7$ & $\mathrm{O} 2$ & $\mathrm{C} 8$ & $\mathrm{O} 1$ & $1.0(7)$ & $\mathrm{C} 23$ & $\mathrm{C} 24$ & $\mathrm{C} 25$ & $\mathrm{C} 26$ & $-1.0(7)$ \\
\hline $\mathrm{C} 7$ & $\mathrm{O} 2$ & $\mathrm{C} 8$ & $\mathrm{~N} 2$ & $-178.3(4)$ & $\mathrm{C} 23$ & $\mathrm{C} 24$ & $\mathrm{C} 25$ & $\mathrm{C} 28$ & $179.5(5)$ \\
\hline $\mathrm{C} 8$ & $\mathrm{~N} 2$ & $\mathrm{C} 10$ & $\mathrm{C} 11$ & $-155.6(4)$ & $\mathrm{C} 24$ & $\mathrm{C} 23$ & $\mathrm{C} 27$ & $\mathrm{~F} 1$ & $-151.0(7)$ \\
\hline $\mathrm{N} 2$ & N1 & $\mathrm{C} 1$ & $\mathrm{C} 2$ & $175.2(4)$ & $\mathrm{C} 24$ & $\mathrm{C} 23$ & $\mathrm{C} 27$ & $\mathrm{~F} 2$ & $92.2(7)$ \\
\hline $\mathrm{N} 2$ & N1 & $\mathrm{C} 1$ & C6 & $-4.7(6)$ & $\mathrm{C} 24$ & $\mathrm{C} 23$ & $\mathrm{C} 27$ & F3 & $-21.1(9)$ \\
\hline $\mathrm{N} 2$ & $\mathrm{~N} 1$ & $\mathrm{C} 12$ & $\mathrm{C} 11$ & $20.9(4)$ & $\mathrm{C} 24$ & $\mathrm{C} 23$ & $\mathrm{C} 27$ & $\mathrm{~F} 2 \mathrm{~A}$ & $45.3(12)$ \\
\hline $\mathrm{N} 2$ & N1 & $\mathrm{C} 12$ & $\mathrm{C} 13$ & $-100.4(4)$ & $\mathrm{C} 24$ & $\mathrm{C} 23$ & $\mathrm{C} 27$ & F3A & $-68.8(10)$ \\
\hline $\mathrm{N} 2$ & $\mathrm{C} 10$ & $\mathrm{C} 11$ & $\mathrm{C} 12$ & $7.0(5)$ & $\mathrm{C} 24$ & $\mathrm{C} 23$ & $\mathrm{C} 27$ & F1A & 169.1(10) \\
\hline $\mathrm{N} 2$ & $\mathrm{C} 10$ & $\mathrm{C} 11$ & $\mathrm{C} 19$ & $178.4(4)$ & $\mathrm{C} 24$ & $\mathrm{C} 25$ & $\mathrm{C} 26$ & $\mathrm{C} 21$ & $0.3(7)$ \\
\hline $\mathrm{C} 10$ & $\mathrm{C} 11$ & $\mathrm{C} 12$ & N1 & $-17.7(4)$ & $\mathrm{C} 24$ & $\mathrm{C} 25$ & $\mathrm{C} 28$ & $\mathrm{~F} 4$ & $0.1(11)$ \\
\hline $\mathrm{C} 10$ & $\mathrm{C} 11$ & $\mathrm{C} 12$ & $\mathrm{C} 13$ & $101.6(4)$ & $\mathrm{C} 24$ & $\mathrm{C} 25$ & $\mathrm{C} 28$ & F5 & $-131.1(10)$ \\
\hline $\mathrm{C} 10$ & $\mathrm{C} 11$ & $\mathrm{C} 19$ & $\mathrm{O} 3$ & $-171.2(4)$ & $\mathrm{C} 24$ & $\mathrm{C} 25$ & $\mathrm{C} 28$ & F6 & $115.3(9)$ \\
\hline $\mathrm{C} 10$ & $\mathrm{C} 11$ & $\mathrm{C} 19$ & $\mathrm{O} 4$ & $9.1(6)$ & $\mathrm{C} 24$ & $\mathrm{C} 25$ & $\mathrm{C} 28$ & F5A & $-163.9(10)$ \\
\hline $\mathrm{C} 11$ & $\mathrm{C} 12$ & $\mathrm{C} 13$ & $\mathrm{C} 14$ & $160.1(4)$ & $\mathrm{C} 24$ & $\mathrm{C} 25$ & $\mathrm{C} 28$ & F6A & $75.3(13)$ \\
\hline $\mathrm{C} 11$ & $\mathrm{C} 12$ & $\mathrm{C} 13$ & $\mathrm{C} 18$ & $-23.1(6)$ & $\mathrm{C} 24$ & $\mathrm{C} 25$ & $\mathrm{C} 28$ & $\mathrm{~F} 4 \mathrm{~A}$ & $-49.7(11)$ \\
\hline $\mathrm{C} 12$ & $\mathrm{~N} 1$ & $\mathrm{C} 1$ & $\mathrm{C} 2$ & $-68.5(5)$ & $\mathrm{C} 26$ & $\mathrm{C} 21$ & $\mathrm{C} 22$ & $\mathrm{C} 23$ & $-0.1(6)$ \\
\hline $\mathrm{C} 12$ & N1 & $\mathrm{C} 1$ & C6 & $111.6(5)$ & $\mathrm{C} 26$ & $\mathrm{C} 25$ & $\mathrm{C} 28$ & $\mathrm{~F} 4$ & $-179.3(9)$ \\
\hline $\mathrm{C} 12$ & $\mathrm{~N} 1$ & $\mathrm{~N} 2$ & $\mathrm{C} 8$ & $146.6(4)$ & $\mathrm{C} 26$ & $\mathrm{C} 25$ & $\mathrm{C} 28$ & F5 & $49.4(11)$ \\
\hline $\mathrm{C} 12$ & $\mathrm{~N} 1$ & $\mathrm{~N} 2$ & $\mathrm{C} 10$ & $-18.4(4)$ & $\mathrm{C} 26$ & $\mathrm{C} 25$ & $\mathrm{C} 28$ & F6 & $-64.2(10)$ \\
\hline $\mathrm{C} 12$ & $\mathrm{C} 11$ & $\mathrm{C} 19$ & $\mathrm{O} 3$ & $-1.0(6)$ & $\mathrm{C} 26$ & $\mathrm{C} 25$ & $\mathrm{C} 28$ & F5A & $16.6(12)$ \\
\hline $\mathrm{C} 12$ & $\mathrm{C} 11$ & $\mathrm{C} 19$ & $\mathrm{O} 4$ & $179.2(4)$ & $\mathrm{C} 26$ & $\mathrm{C} 25$ & $\mathrm{C} 28$ & F6A & $-104.1(12)$ \\
\hline
\end{tabular}




$\begin{array}{llllllllll}\mathrm{C} 12 & \mathrm{C} 13 & \mathrm{C} 14 & \mathrm{C} 15 & 176.3(4) & \mathrm{C} 26 & \mathrm{C} 25 & \mathrm{C} 28 & \mathrm{~F} 4 \mathrm{~A} & 130.9(10) \\ \mathrm{C} 12 & \mathrm{C} 13 & \mathrm{C} 18 & \mathrm{C} 17 & -177.0(4) & \mathrm{C} 27 & \mathrm{C} 23 & \mathrm{C} 24 & \mathrm{C} 25 & -178.5(5) \\ \mathrm{C} 13 & \mathrm{C} 14 & \mathrm{C} 15 & \mathrm{Br} 1 & -177.7(3) & \mathrm{C} 28 & \mathrm{C} 25 & \mathrm{C} 26 & \mathrm{C} 21 & 179.8(5) \\ \mathrm{C} 13 & \mathrm{C} 14 & \mathrm{C} 15 & \mathrm{C} 16 & 0.7(6) & & & & & \end{array}$

Table S7. Hydrogen Atom Coordinates $\left(\AA \times 10^{4}\right)$ and Isotropic Displacement Parameters $\left(\AA^{2} \times 10^{3}\right)$ for $3 h a$.

\begin{tabular}{|c|c|c|c|c|}
\hline \multicolumn{2}{|c|}{$\operatorname{Atom} x$} & \multirow{2}{*}{$\begin{array}{l}\boldsymbol{y} \\
99.89\end{array}$} & \multirow{2}{*}{$\begin{array}{l}z \\
4154.42\end{array}$} & \multirow{2}{*}{$\begin{array}{l}\mathbf{U}(\mathbf{e q}) \\
43\end{array}$} \\
\hline $\mathrm{H} 2$ & 1185.1 & & & \\
\hline H3 & 814.88 & -487.99 & 2318.08 & 57 \\
\hline H4 & 2049.45 & 348.02 & 1186.87 & 68 \\
\hline H5 & 3718.8 & 1637.55 & 1914.31 & 72 \\
\hline H6 & 4121.41 & 2235.75 & 3740.5 & 55 \\
\hline $\mathrm{H} 7 \mathrm{~A}$ & 7158.93 & 1063.88 & 6184.6 & 66 \\
\hline H7B & 6774.94 & 822.36 & 7326.72 & 66 \\
\hline $\mathrm{H} 7 \mathrm{C}$ & 7478.62 & 2257.78 & 7113.43 & 66 \\
\hline H10 & 4373.51 & 4417.41 & 5935.11 & 34 \\
\hline H12 & 1287.11 & 2627.23 & 4982.94 & 28 \\
\hline H14 & 308.66 & 724.42 & 5856.84 & 30 \\
\hline H16 & 691.66 & 595.09 & 9110.06 & 39 \\
\hline H17 & 2181.93 & 2336.68 & 9237.36 & 44 \\
\hline H18 & 2766.95 & 3222.6 & 7687.94 & 39 \\
\hline $\mathrm{H} 20 \mathrm{~A}$ & 1619.4 & 7993.87 & 5933.17 & 41 \\
\hline $\mathrm{H} 20 \mathrm{~B}$ & 2872.53 & 8686.07 & 5883.49 & 41 \\
\hline $\mathrm{H} 22$ & 1326.66 & 6821.66 & 7705.37 & 35 \\
\hline $\mathrm{H} 24$ & 3371.33 & 8300.37 & 10422.43 & 40 \\
\hline $\mathrm{H} 26$ & 4156 & 9294.73 & 7507.28 & 43 \\
\hline
\end{tabular}


Table S8. Atomic Occupancy for 3 ha.

$\begin{array}{llllll}\text { Atom } & \text { Occupancy } & \text { Atom } & \text { Occupancy } & \text { Atom } & \text { Occupancy } \\ \text { F1 } & 0.619(9) & \text { F2 } & 0.619(9) & \text { F3 } & 0.619(9) \\ \text { F4 } & 0.547(12) & \text { F5 } & 0.547(12) & \text { F6 } & 0.547(12) \\ \text { F2A } & 0.381(9) & \text { F3A } & 0.381(9) & \text { F1A } & 0.381(9) \\ \text { F5A } & 0.453(12) & \text { F6A } & 0.453(12) & \text { F4A } & 0.453(12)\end{array}$

\section{Experimental}

Single crystal of $\mathrm{C}_{27} \mathrm{H}_{19} \mathrm{BrF}_{6} \mathrm{~N}_{2} \mathrm{O}_{4}$ (3ha) was recrystallized from [petroleum ether and dichloromethane] mounted in inert oil and transferred to the cold gas stream of the diffractometer.

\section{Crystal Structure Determination of [3ha]}

Crystal Data for $\mathrm{C}_{27} \mathrm{H}_{19} \mathrm{BrF}_{6} \mathrm{~N}_{2} \mathrm{O}_{4}$ (3ha) $(M=629.35 \mathrm{~g} / \mathrm{mol})$ : monoclinic, space group $\mathrm{P} 21$ (no. 4), $a=11.661(2) \AA, b=9.1802(18) \AA, c=12.632(3) \AA, \beta=100.26(3)^{\circ}, V=1330.6(5) \AA^{3}$, $Z=2, T=153.15 \mathrm{~K}, \mu(\mathrm{MoK} \alpha)=1.623 \mathrm{~mm}^{-1}$, Dcalc $=1.571 \mathrm{~g} / \mathrm{cm}^{3}, 14984$ reflections measured $\left(5.242^{\circ} \leq 2 \Theta \leq 54.952^{\circ}\right), 6032$ unique $\left(R_{\text {int }}=0.0440, \mathrm{R}_{\text {sigma }}=0.0472\right)$ which were used in all calculations. The final $R_{1}$ was 0.0417 (I $\left.>2 \sigma(\mathrm{I})\right)$ and $w R_{2}$ was 0.0984 (all data). 